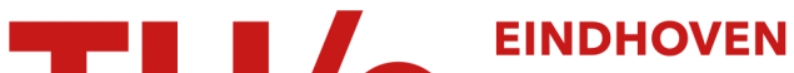 \\ UNIVERSITY OF \\ TECHNOLOGY
}

\section{Linear programming over an infinite horizon}

Citation for published version (APA):

Evers, J. J. M. (1973). Linear programming over an infinite horizon. [Phd Thesis 2 (Research NOT TU/e / Graduation TU/e), Mathematics and Computer Science]. Technische Hogeschool Eindhoven.

https://doi.org/10.6100/IR88742

DOI:

10.6100/IR88742

Document status and date:

Published: 01/01/1973

\section{Document Version:}

Publisher's PDF, also known as Version of Record (includes final page, issue and volume numbers)

\section{Please check the document version of this publication:}

- A submitted manuscript is the version of the article upon submission and before peer-review. There can be important differences between the submitted version and the official published version of record. People interested in the research are advised to contact the author for the final version of the publication, or visit the $\mathrm{DOI}$ to the publisher's website.

- The final author version and the galley proof are versions of the publication after peer review.

- The final published version features the final layout of the paper including the volume, issue and page numbers.

Link to publication

\section{General rights}

Copyright and moral rights for the publications made accessible in the public portal are retained by the authors and/or other copyright owners and it is a condition of accessing publications that users recognise and abide by the legal requirements associated with these rights.

- Users may download and print one copy of any publication from the public portal for the purpose of private study or research.

- You may not further distribute the material or use it for any profit-making activity or commercial gain

- You may freely distribute the URL identifying the publication in the public portal.

If the publication is distributed under the terms of Article 25fa of the Dutch Copyright Act, indicated by the "Taverne" license above, please follow below link for the End User Agreement:

www.tue.nl/taverne

Take down policy

If you believe that this document breaches copyright please contact us at:

openaccess@tue.nl

providing details and we will investigate your claim. 


\section{LINEAR PROGRAMMING OVER AN INFINITE HORIZON}

J.J.M.Evers 
LINEAR PROGRAMMING OVER

AN INFINITE HORIZON

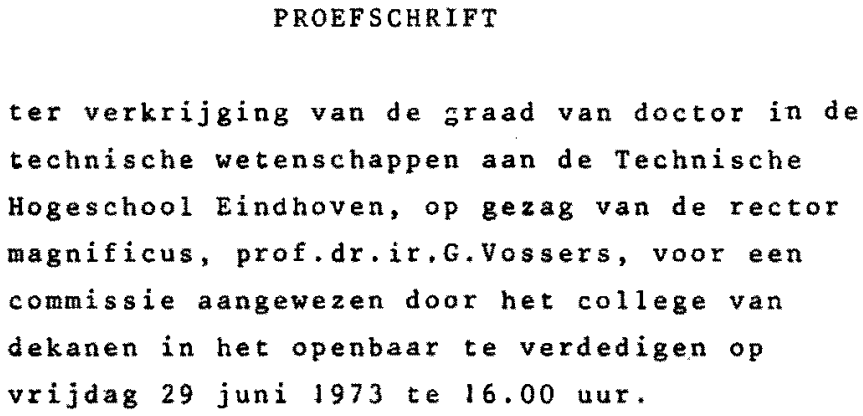


Dit proefschrift is goedgekeurd door de promotoren:

prof.dr.J.F.Benders en

dr.ir.M.L.J.Hautus. 
CONTENTS.

CHAPTER 1: Linear programming in growth models over an infinite horizon.

CHAPTER 2 : Mathematical formulation of the linear programming system.

CHAPTER 3 : Directedness, feasibility, and regularity.

CHAPTER 4 : Partial objective functions.

CHAPTER 5 : Inferiority.

CHAPTER 6: Optimality.

CHAPTER 7 : Parametric properties.

CHAPTER 8 : Paths of equilibrium.

CHAPTER 9 : Semi equilibrium paths.

CHAPTER 10: Equivalent linear programming problems over a finite horizon.

REFERENCES

LIST OF SYMBOLS 


\section{LINEAR PROGRAMMING IN GROWTH MODELS OVER AN INFINITE HORIZON}

\subsection{Introduction.}

Practically all applications of the linear programing theory to growth models in the economy $(2: 265)$, (9:254), have in common that they limit themselves to a program over a finite horizon. Into many models, however, the fixation of a horizon introduces a certain arbitrariness (4:105) which can be avoided by formulating the problem over an infinite horizon. Then, mathematically speaking, a linear programming problem arises in an infinite dimensional space. This study presents a mathematical analysis of such a problem, which results in a general solving procedure. In this analysis we assume a particular characteristic with regard to the structure of the linear programing problem.

In this chapter four growth models will be discussed in macro economical terms, in order to arrive at a first formulation of the problem in an economical context, and to show that practically every realistic linear growth problem can be formulated into a model possessing the specific structure presumed in the mathematical treatment. As such, the models discussed are of secondary importance. The chapter will be concluded with a brief reference to the most important results.

Since chapter 2 presents a formal mathematical definition of the problem, and since its mathematical elaboration is not connected with the growth models outlined above, those exclusively interested in the mathematical treatment can skip this chapter without objection.

\subsection{Growth model I.}

We consider an economy with $m_{1}$ goods and $n$ production processes. The production processes are specified as follows:

a) The production processes are executed in a sequence of periods of equal duration. The input received at the beginning of a period results in an output available at the end. 
b) The production processes are linear: for every period, the input and output of each production process is proportional to the level of activity at which it is executed during the period.

c) For each production process, the proportion between the quantity of input and output and the level of activity is constant for all periods.

We shall represent the activity levels at which the production processes are executed during the periods $t=0,1, \ldots$ sy a sequence of non-negative n-dimensional vectors $x(0), x(1), \ldots$, in which $x_{j}(t)$ is the activity level for the $j$-th production process, during the t-th period. The suppositions b) and $c$ ) imply that, for each sequence of activity levels $x(0), x(1), \ldots$, the corresponding quantities of input and output may be expressed as follows:

$$
\begin{aligned}
& \mathrm{Bx}(t), \quad t \geq 0, \\
& \mathrm{Ax}(t), \quad t \geq 0,
\end{aligned}
$$

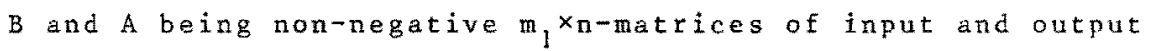
coefficients; $i . e: b_{i j} x_{j}(t)$ and $a_{i j} x_{j}(t)$ are the quantities of input and output resp. of the $i-t h$ goods if the $j-t h$ production process during the t-th period is executed at an activity level $x_{j}(t)$. According to supposition a). the input $b_{i j} x_{j}(t)$ is absorbed at the beginning of period $t$ and the output $a_{i j} x_{j}(t)$ is available at the end of this period.

With regard to the transfer of goods between the various production processes we further introduce the following suppositions:

d) The transfer of goods between the processes, takes place timelessly during the changing of periods.

e) Surpluses are allowed.

f) Surpluses occurring during one change of period are not available during the following changes of periods. 
If no goods from without are conveyed towards or from this economy, then the latter three suppositions imply that with a given vector of initial activity levels $x(0)$, a sequence of activity levels $x(1), x(2), \ldots$ is feasible if and only if the following inequalities are satisfied:

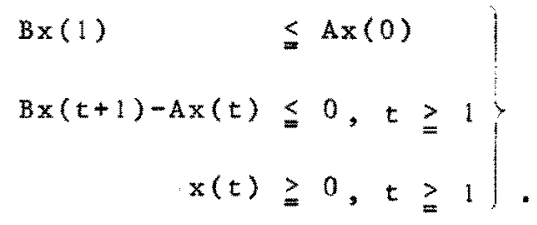

We observe that the conditions e) and f) appear more limiting than in reality they are. For the preservation of surpluses during a period as such, can. again be formulated as a special production process.

To the system described thus far, we shall add a number of elements, whereas the conditions a) to f) will remain valid.

Firstly one may assume that goods from without are made available for the system. In this connection, for instance the factor labour might, in a certain maner, be taken into account. We assume, that this availability of goods has a content of $\alpha^{t} f$, for each change of period $(t-1, t)$. In which assumption $\alpha>0$ represents a growth factor and $f$ a non-negative m-dimensional vector. Under this extension system (1.2.2) takes the following form:

$$
\begin{aligned}
B \times(1) & \leqq \alpha f+A \times(0) \\
B \times(t+1)-A x(t) & \leqq a^{t+1} f, \quad t \geqq 1 \\
x(t) & \geqq 0 \quad, \quad t \geqq 1
\end{aligned}
$$

We now turn our attention towards the consumption aspect. We distinguish two kinds of consumption: autonomous consumption and endogeneous consumption. Autonomous consumption may be interpreted as those quantities of goods which are necessary to meet the primary needs. Endogeneous consumption may be interpreted as the extra. 
We assume that, for each change of period $(t-1, t)$, the autonomous consumption is $\beta^{t} g$; herein $\beta>0$ represents a growth factor and $g$ a non-negative m,-simensional vector. Under this addition, system (1.2.3) takes the form of:

$$
\left.\begin{array}{rlrl}
B \times(1) & \leqq \alpha f-B g+A x(0) & \\
B \times(t+1)-A x(t) & \leqq \alpha^{t+1} f-\beta^{t+1} g, t \geqq 1 \\
x(t) & \geqq 0 . & t \geqq 1
\end{array}\right\} .
$$

Thus, given the initial activity levels $x(0)$, a sequence of activity levels $x(1), x(2), \ldots$ is feasible if and only if (1.2.4) is satisfied.

With regard to the process of endogeneous consumption we introduce the following suppositions comparable with a), b) and cy:

g) The process of free consumption takes place in a sequence of periods of the same duration as that of the production processes.

h) The process of free consumption may consist of various subprocesses. For each subprocess the input of goods is proportional to its level of activity.

i) For each subprocess the proportion of input and the level of consumption activity remains the same for all periods.

The latter three suppositions are elaborated in a similar manner as the suppositions $a$ ), b) and c). Assuming that there are $k$ subprocesses for endogeneous consumption, and representing the activity levels of these subprocesses by a sequence of non-negative $k$-dimensional vectors $x^{c}(1), x^{c}(2), \ldots$, then, with the help of a non-negative $m_{1} \times k-m a t r i x B^{c}$ of consumption coefficients, the corresponding quantity of input may be expressed:

$$
B^{c} x^{c}(t), \quad t \geqq 1
$$

Combining $(1.2 .4)$ and $(1.2 .5)$, we may conclude that, under the 
conditions a) to i), with a given $x(0)$, a sequence of activity levels $\left(x(1), x^{c}(1)\right),\left(x(2), x^{c}(2)\right), \ldots$ is feasible if and only if

$$
\left.\begin{array}{rl}
B x(1)+B^{c} x^{c}(1) & \leq \alpha f-B g+A x(0) \\
B x(t+1)+b^{c} x^{c}(t+1)-A x(t) & \leq \alpha^{t+1} f-\beta^{t+1} g, t \geq 1 \\
x(t), x^{c}(t) & \geq 0 \quad, t \geq 1
\end{array}\right\}(1.2 .6)
$$

is satisfied.

We complete the introduction of engogeneous consumption by addition of a linear utility function. We shall assume that the utility of each sequence of levels of consumption activities $x^{c}(1), x^{c}(2), \ldots, x^{c}(T), \ldots$ can be expressed as follows:

$$
\sum_{t=1}^{T} \pi^{t} p^{*} x^{c}(t)
$$

where:

- $p$ is a non-negative k-dimensional vector and

- $\pi$ a positive discount factor, in which the appreciation of a succeeding period is expressed in relation to the preceding period. So, generally $\pi$ will be smaller than one.

Starting from the supposition that the economy described above does no cease to exist, the following problem arises: How many periods will the utility function (1.2.7) of a feasible sequence of activity levels $\left(x(1), x^{c}(1)\right),\left(x(2), x^{c}(2)\right), \ldots$ have to cover for an adequate valuation of such a sequence to be obtained? Clearly, each fixation of the number of periods covered by the valuation, or differently put, fixation of the horizon, is bound to introduce a certain arbitrariness. This arbitrariness can be efficiently avoided by effectuating the valuation over an infinite number of periods. This results in the following expression:

$$
\sum_{t=1}^{\infty} \pi^{t} p^{\prime} x^{c}(t):=\lim _{T \rightarrow \infty} \sum_{t=1}^{T} \pi^{t} p^{\prime} x^{c}(t) .
$$


Now, a particular difficulty may present itself. For it is very well possible that, for a number of feasible sequences of activity levels $\left(x(1), x^{c}(1)\right),\left(x(2), x^{c}(2)\right), \ldots$, the 1 imit $(1.2 .8)$ does not exist. To illustrate this possibility, we consider the case that there is no autonomous consumption (i.e.: $g=0$ ) and that, for certain $x(0)$, system $(1.2 .6)$ possesses a solution of the form

$$
\left.\begin{array}{l}
x(t):=\alpha^{t} x^{1} \\
x^{c}(t):=\alpha^{t} x^{2}
\end{array}\right\}, \quad t \geqq 1 .
$$

For such a feasible sequence, we have:

$$
\sum_{t=1}^{T} \pi^{t} p^{i} x^{c}(t)=(\alpha \pi) \frac{1-(\alpha \pi)^{T+1}}{1-(\alpha \pi)} p^{\prime} x^{2}, \quad T \geqq 1 .
$$

If $p^{\prime} x^{2}>0$ and if $\alpha \pi \geqslant 1$, then it appears that the sequence of numbers defined by $(1.2 .10)$ has no upper bound. In that case, the expression $(1.2 .8)$ cannot represent a sensible utility function. This difficulty can be eliminated by choosing the positive coefficient $\pi$ so small that $\alpha \pi<1$.

Apart from the complication as sketched above, the optimization aspect in this economy can be roughly formulated as follows:

Given the initial intensities $x(0)$, find a sequence of activity levels $\left(x(1), x^{c}(1)\right),\left(x(2), x^{c}(2)\right), \ldots$ which satisfies $(1.2 .6)$ and for which the limit $(1.2 .8)$ attains a maximum value.

Finally, we shall give the model consisting of the inequalities $(1.2 .6)$ and the utility function $(1.2 .7)$ and $(1.2 .8)$ a more general form.

Firsty, we write the left hand side of $(1.2 .6)$ in the less specified form:

$$
p^{t} f(t), t \geq 1 \text {, }
$$

in which $\rho$ is a positive growth factor and $f(1), f(2), \ldots$ a se- 
quence of $m_{1}$-dimensional vectors for which non-negative vectors $\underline{f}$ and $\bar{f}$ are supposed to exist such that

$$
-\underline{f} \leqq f(t) \leqq \bar{f}, \quad t \geqq 1 .
$$

Thus, $(1.2 .6)$ takes the form:

$$
\left.\begin{array}{rl}
B x(1)+B^{c} x^{c}(1) & \leqq p f(1)+A x(0), \\
B x(t+1)+B^{c} x^{c}(t+1)-A x(t) & \leqq \rho^{t+1} f(t+1), t \geq 1 ; \\
x(t), x^{c}(t) & \geqq 0, t \geqq 1
\end{array}\right\}
$$

With the help of two non-negative $m_{1} \times(n+k)$-matrices:

$$
\left.\begin{array}{l}
\bar{B}:=\left(B, B^{C}\right) \\
\bar{A}:=(A, 0)
\end{array}\right\}
$$

this system can be witten:

$$
\left.\begin{array}{rlrl}
B \times(1) & \leqq \rho f(1)+A x(0) & \\
B x(t+1)-A x(t) & \leqq p^{t+1} f(t+1), t \geq 1 \\
\bar{x}(t) & \geqq 0, & t \geqq 1
\end{array}\right\},
$$

where $\bar{x}(0), \bar{x}(1), \ldots$ is a sequence of $(n+k)$-dimensional vectors, which correspond with the activity levels $\left(x(0), x^{c}(0)\right),\left(x(1), x^{c}(1)\right), \ldots$.

The utility functions $(1.2 .7)$ and $(1.2 .8)$ will be written in the less specified form:

$$
\begin{aligned}
& \sum_{t=1}^{T} \pi^{t} \bar{p}(t) \cdot \bar{x}(t), \quad T \geq 1, \\
& \sum_{t=1}^{\infty} \pi^{t} \bar{p}(t) \cdot \bar{x}(t),
\end{aligned}
$$


in which $\bar{p}(1), \bar{p}(2), \ldots$ is a sequence of $m_{1}$-dimensional vectors for which non-negative vectors $p$ and $\underset{p}{q}$ are supposed to exist such that:

$$
-p \leqq \bar{p}(t) \leqq \stackrel{q}{p}, \quad t \geqq 1
$$

Since negative components are permitted in the vectors $\bar{p}(1), \bar{p}(2), \ldots$ we shall use the more general term objective functions, for the expressions $(1.2 .14)$ and $(1.2 .15)$.

Growth model $I$, consisting of the inequalities (1.2.13) and the objective functions $(1.2 .14)$ and $(1.2 .15)$, will be the point of departure for the growth models now to be discussed.

\subsection{Growth mode1 II.}

We now turn our attention towards durable goods, or briefly, durables. In comparison with the goods of model I, further to be called non-durables, the durable goods possess some characteristic properties specified as follows:

a) The durables which are necessary for a production process, will be adopted at the beginning of a period and will become free at the end of the period.

b) The quantity of durables which are used in a production process, is proportional to the activity level of this process.

c) The proportion between the durables used in a production process, and the level of activity of the process, is the same for all periods.

The formation and the process of obsolescence of all sorts of durables will be specified as follows:

d) All durables are formed timelessly outoff the non-durables on the moments of period change.

e) The quantity of non-durables used for the formation of durables is proportional to the quantity of these goods. 
f) The proportions of the quantities mentioned at e) are constant for all periods.

g) All durables have a finite curability. The curve of obsolescence is the same for all periods. Moreover, durables cannot change in type.

h) There is no exogeneous supply of removal of capacity goods.

The transfer of durables is supposed to be of the same nature as specified in $1.2-d, e, f$.

First we elaborate the suppositions 1.3-d,g. For the sake of simplicity, we here assume that the durability of durable goods is three periods at most. Let $L$ be the number of sorts of durables, then, during each period $t$, this economy contains the following durables:

- durables formed at the moment of period change $(t-1, t)$; the quantity of these is expressed by a non-negative L-diansional vector $z(t ; 0)$,

- durables formed at the moment of period change $(t-2, t-1)$; represented by a non-negative L-dimensional vector $z(t ; 1)$,

- durables formed at the moment of period change $(t-3, t-2)$; represented by a non-negative L-dimensional vector $x(t ; 2)$.

With the help of two non-negative $\mathrm{L} \times \mathrm{L}-\mathrm{d}$ imensional matrices $\gamma(1)$ and $Y(2)$, for every period $t$, the actual quantity of durables (viz. $1.3-g$ ) can be expressed by

$$
z(t ; 0)+\gamma(1) z(t ; 1)+\gamma(2) z(t ; 2)
$$

The suppositions $1.2-e, f$ and the definition of the vectors $z(t ; 0), z(t ; 1), z(t ; 2), t \geq 0$, imply the inequalities:

$$
\left.\begin{array}{l}
z(t+1 ; 1) \leqq z(t ; 0) \\
z(t+1 ; 2) \leqq z(t ; 1)
\end{array}\right\}, \quad t \geqq 0 .
$$


For the elaboration of the suppositions 1.3-a to $1: 3-f$, we start from the inequalities $(1.2 .13)$ of growth model I:

$$
\begin{array}{rlrl}
B \times(1) & \leqq p f(1)+A x(0) & & \\
B x(t+1)-A x(t) & \leqq \rho^{t+1} f(t+1), t \geqq 1 \\
\bar{x}(t) & \geqq 0 \quad, t \geqq 1
\end{array} \mid, \quad(1.3 \cdot 3)
$$

with $m_{1}$ types of non-durables and $n_{1}:=n+k$ processes.

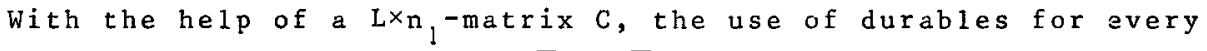
sequence of activity levels $\bar{x}(1), \bar{x}(2), \ldots$, can be expressed by:

$$
C \bar{x}(t), \quad t \geqq 1
$$

With the help of a non-negative matrix $D$ the volume of nondurables used for the formation of durables can be expressed by:

$$
\mathrm{Dz}(t ; 0), \quad t \geq 1
$$

The supposition $1.2-a, d, g$ and $1.3-a, d$ imply that for the first period we may join the expressions (1.3.1) to (1.3.5) in the following inequalities:

$$
\left.\begin{array}{rl}
\mathrm{Bx}(1)+D z(1 ; 0) & \leqq \rho \mathrm{f}(1)+\overline{\mathrm{A}} \mathrm{x}(0) \\
C \overline{\mathrm{x}}(1)-z(1 ; 0) \gamma(1) z(1 ; 1)-\gamma(2) z(1 ; 2) & \leqq 0 \\
z(1 ; 1) & \leqq z(0 ; 0) \\
z(1 ; 2) & \leqq z(0 ; 1) \\
\bar{x}(1), z(1 ; 0), z(1 ; 1), z(1 ; 2) & \geqq 0
\end{array}\right\}(1.3 .6)
$$

and for the succeeding periods: 
$B \times(t+1)+D z(t+1 ; 0$

$$
-A x(t) \leqq \rho^{t+1} f(t+1) !
$$

$C x(t+1)-z(t+1 ; 0)-\gamma(1) z(t+1 ; 1)-\gamma(2) z(t+1 ; 2) \leqq 0$

$$
\begin{aligned}
z(t+1 ; 1)-z(t ; 0) & \leqq 0 \\
z(t+1 ; 2)-z(t ; 1) & \leqq 0 \\
\bar{x}(t), z(t ; 0), z(t ; 1), z(t ; 2) \geqq 0 & \vdots
\end{aligned}
$$

Thus, given $(\bar{x}(0), z(0 ; 0), z(0 ; 1))$, a sequence $(\bar{x}(t), z(t ; 0), z(t ; 1), z(t ; 2)), t=1,2, \ldots$ is feasible if and on $1 y$ if $(1.3 .6)$ and $(1.3 .7)$ are satisfied.

With the help of the $\left(m_{1}+L+L+L\right) \times\left(n_{1}+L+L+L\right)-m a t r i c e s$

$$
\mathrm{B}:=\left[\begin{array}{cccc}
\bar{B}, & \mathrm{D}, & 0, & 0 \\
\mathrm{C}, & -\mathrm{I},-\gamma(1),-\gamma(2) \\
0, & 0, & \mathrm{I}, & 0 \\
0, & 0, & 0, & \mathrm{I}
\end{array}\right] \quad \mathrm{A}:=\left[\begin{array}{cccc}
\overline{\mathrm{A}}, & 0, & 0, & 0 \\
0, & 0, & 0, & 0 \\
0, & \mathrm{I}, & 0, & 0 \\
0, & 0, & \mathrm{I}, & 0
\end{array}\right]
$$

and with the help of the sequence of $\left(m_{1}+L+L+L\right)$-dimensional vectors defined by

$$
\dddot{E}(t):=\left[\begin{array}{c}
f(t) \\
0 \\
0 \\
0
\end{array}\right], \quad t \geqq 1
$$

the inequalities $(1.3 .7)$ can be written:

$$
\begin{aligned}
& \text { Bरx(1) } \leqq \rho \tilde{f}(1)+\hat{A x}(0) \\
& \left.\begin{array}{rl}
\tilde{B x}(t+1)-\tilde{A x}(t) \leqq p^{t+1} \underline{x}(t+1), & t \geq 1 \\
\tilde{x}(t) \geqq 0 \quad, t \geq 1
\end{array}\right\} .
\end{aligned}
$$


where $\mathrm{x}(0), \tilde{x}(1), \ldots$, is a sequence of $\left(n_{1}+L+L+L\right)-d i m e n s i o n a 1$ vectors which corresponds with the sequence $(\bar{x}(t), z(t ; 0), z(t ; 1), z(t ; 2)), t=0,1,2, \ldots$ appearing in $(1.3 .6)$ and (1.3.7). So, the vectors $\tilde{x}(t), t \geqq 0$ represent quantities of a different nature. Therefore, we shall use the more general. term state vectors.

When we define the sequence of $\left(n_{1}+L+L+L\right)-d i m e n s i o n a l$ vectors $\stackrel{p}{p}(1), \tilde{p}(2), \ldots$ by:

$$
\tilde{\mathrm{p}}(\mathrm{t}):=\left[\begin{array}{c}
\overline{\mathrm{p}}(\mathrm{t}) \\
0 \\
0 \\
0
\end{array}\right], t \geq 1
$$

$\bar{p}(1), \bar{p}(2), \ldots$ being the sequence of vectors of the objective functions $(1.2 .14)$ and $(1.2 .15)$, then the corresponding objective functions of a sequence $x(1), x(2), \ldots$ which satisfies $(1.3 .19)$, can be written:

$$
\begin{aligned}
& \sum_{t=1}^{T} \pi^{t \tilde{p}(t) \cdot \tilde{x}(t), \quad T \geq 1,} \\
& \sum_{t=1}^{\infty} \pi^{t \tilde{p}(t) \cdot \tilde{x}(t) .}
\end{aligned}
$$

Clearly, the optimization aspect may be formulated in the same manner as in growth model $T$.

The system of inequalities (1.3.10) together with the objective functions $(1.3 .12)$ and $(1.3 .13)$ form growth model II. We observe that this model has the same form as growth model I. Further we observe that the definitions $(1.3 .8)$ and $(1.3 .9)$ imply the following:

i) Each row vector $\hat{b}_{i}$; of matrix $\tilde{B}_{\text {in }}$ wh contains one or more negative components, corresponds with a non-negative row 
vector $\tilde{a}_{i}$ of matrix $\AA$ and with non-negative components $\widetilde{f}_{i}(t)$ of the vectors $\tilde{f}(1), \tilde{f}(2), \ldots$.

j) Matrix A contains no negative components.

1.4 Growth mode1 III.

Now we shall add import and export facilities. These are specified as follows:

a) The processes of import and export are executed in the same sequence of periods as the production processes. The quantity of goods to be imported and exported is determined at the beginning of a period; the actual import and export takes place timelessly at the end of the period.

b) The quantity of goods which are used for the effectuation of import and export (for instance transport capacity) is taken up at the beginning of a period, and is proportional to the quantity of imported and exported goods.

c) Import and export take place at fixed prices. Import prices are not lower than export prices.

d) The reserve of payments at the end of a period is composed of reserve of payments at the end of the preceeding period, inultiplied by an interest factor, increased by the value of the export and decreased by the value of the import at the end of the period.

e) The reserve of payments cannot be negative.

f) The proportion of the quantities mentioned at b), the import and export prices and the interest factor appearing in d) are the same for al1 periods.

We take growth model II as the point of departure:

$$
\begin{aligned}
\tilde{B x}(1) & \leqq p \tilde{f}(1)+\tilde{A x}(0) \\
\tilde{B x}(t+1)-\tilde{A x}(t) & \leqq \rho^{t+1 \tilde{f}(t+1),}, t \geqq 1 \\
\tilde{x}(t) & \geqq 0 .
\end{aligned}
$$$$
(1.4 \cdot 1)
$$ 
where:

- $\tilde{x}(0), \tilde{x}(1), \ldots$ and $\tilde{f}(1), \tilde{f}(2), \ldots$ are sequences of $n_{2}$ and $m_{2} d i-$ mensional vectors $\left(m_{2}:=m_{1}+L+L+L\right.$ and $\left.n_{2}:=n_{1}+L+L+L\right)$,

$-\widetilde{A}$ and $\tilde{B}$ are $m_{2} \times n_{2}$-matrices which possess the properties $1.3-i$ and $1 \cdot 3-j$.

For the sake of simplicity, we shall here assume that all types of goods can be imported and exported; the quantities will be denoted by the sequences of $m_{2}$-dimensional vectors $x^{i}(0), x^{i}(1), \ldots$ and $x^{e}(0), x^{e}(1), \ldots, x^{i}(t)$ being the quantity of imported goods at the end of period $t$ and $x^{e}(t)$ the export quantity.

Supposition 1.4-b implies that the quantity of goods used for the effectuation of import and export can be expressed by:

$$
\left.\begin{array}{ll}
c^{i} x^{i}(t), & t \geqq 0 \\
c^{e} x^{e}(t), & t \geqslant 0
\end{array}\right\}
$$

where $c^{i}$ and $c^{e}$ are non-negative $m_{2} \times m_{2}$-matrices.

Supposition $1.4-a$ implies that $(1.4 .1)$ and $(1.4 .2)$ can be combined into the system:

$$
\begin{aligned}
& \hat{B x}(1)+C^{i} x^{i}(1)+C^{e} x^{e}(1) \leqq \rho^{2}(1)+\hat{A} x(0)+x^{i}(0)-x^{e}(0) \\
& B x(t+1)+C^{i} x^{i}(t+1)+C^{e} x^{e}(t+1)-A x(t)-x^{i}(t)+x^{e}(t) \leqq 0^{t+1} t(t+1), t \geqq 1 \\
& x(t), x^{i}(t), x^{e}(t) \geq 0 \\
& \text {, } \mathrm{t} \geqq 0
\end{aligned}
$$

The suppositions $1.4-\mathrm{c}$ to $1.4-f$ are elaborated as follows. The reserves of payment at the end of the periods $t=0,1,2, \ldots$ can be represented by a sequence of non-negative numbers $r(0), r(1), \ldots$. Denoting the import and export by non-negative $\mathrm{m}_{2}$-dimensional vectors $q^{i}$ and $q^{e}$, and the interest factor by a number $\alpha>1$, then supposition 1.4-d gives rise to the following inequalities: 


$$
\left.\begin{array}{r}
r(1) \leqq \alpha r(0)-q^{i} x^{i}(0)+q^{e} \cdot x^{e}(0) \\
r(t+1)-\alpha r(t)+q^{i} x^{i}(t)-q^{e}, x^{e}(t) \leqq 0, \quad t \geqq 1 \\
r(t), x^{i}(t), x^{e}(t) \geqq 0
\end{array}\right\} \quad(1.4 .4)
$$

Now, we may conclude that in such an economy, given the initial quantities $\left(x(0), r(0), x^{i}(0), x^{e}(0)\right)$, a sequence $\left(\tilde{x}(1), r(1), x^{i}(1), x^{e}(1)\right),\left(x(2), r(2), x^{i}(2), x^{e}(2)\right), \ldots$ is feasible if and only if simultaneously the inequalities (1.4.3) and $(1.4 .4)$ are satisfied.

With the help of the $\left(m_{2}+1\right) \times\left(n_{2}+1+m_{2}+m_{2}\right)$-matrices

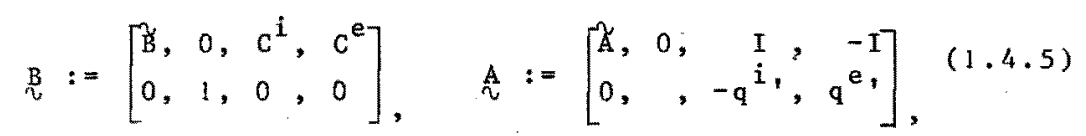

and with the help of a sequence of $\left(\mathrm{m}_{2}+1\right)$-dimensional vectors $\underset{\mathrm{f}}{\mathrm{f}}(1), \underset{\mathrm{f}}{\mathrm{f}}(2), \ldots$ defined by:

$$
f(t):=\left[\begin{array}{c}
f(t) \\
0
\end{array}\right], \quad t \geqq 1
$$

the systems $(1.4 .3)$ and $(1.4 .4)$ may be combined in the system:

$$
\begin{aligned}
& \mathrm{N}_{\mathrm{X}}(1) \quad \leqq p f(1)+\mathrm{Ax}(0)
\end{aligned}
$$

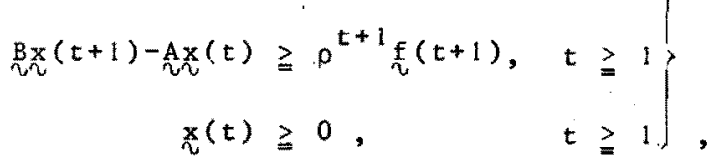

where $\underset{x}{x}(0), \underset{2}{x}(1), \ldots$ is a sequence of $\left(n_{2}+1+m_{2}+m_{2}\right)$-dimensiona 1 vectors which corresponds with the sequence $\left(x(0), r(0), x^{i}(0), x^{e}(0)\right),\left(x(1), r(1), x^{i}(1), x^{e}(1)\right), \ldots$.

When we define the sequence of $\left(n_{2}+1+m_{2}+m_{2}\right)$-dimensional vectors 


$$
\mathcal{f}(t):=\begin{aligned}
& \tilde{p}(t) \\
& 0 \\
& 0 \\
& 0
\end{aligned}, \quad t \geq 1
$$

$\tilde{\mathrm{p}}(1), \hat{\mathrm{p}}(2), \ldots$ being the sequence of vectors of the objective functions (1.3.12) and (1.3.13), then the corresponding objective functions of a sequence $\underset{\mathrm{x}}{\mathrm{x}(1)}$, $\mathrm{x}(2), \ldots$ which satisfies (1.4.7), can be written

$$
\begin{aligned}
& \sum_{t=1}^{I} \pi^{t} p(t)^{\prime} x(t), T \geq 1, \\
& \sum_{t=1}^{\infty} \pi \pi^{t} p(t)^{\prime} x(t) .
\end{aligned}
$$

System (1.4.7) together with the objective functions (1.4.9) and (1.4.10) form growth model III. This model has the same form of growth models I and II.

clearly, the definitions $(1.4 .5)$ and (1.4.8) and property $1.3-j$ imply that growth model III possesses the following property:

g) Each column vector d.j of matrix fhich contains one or more negative components, corresponds with a non-negative column vector R. $j$ of matrix $\underset{\sim}{B}$, and with non-positive components

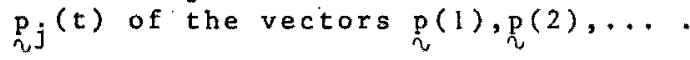

\subsection{Growth model IV.}

Now, the suppositions $1.2-c, 1.3-c$, f and $1.4-g$ will be weakened by introduction of the possibility of cyclic change, as for instance caused by the influence of the seasons. For the sake of simplicity, we here suppose that the cycle consists of two phases: the first and the second half of the year. We put model III as the point of departure. 
The state vectors of model III will be represented by a sequence of pairs of non-negative $n_{3}$-dimensional vectors $\left(x^{1}(0), x^{2}(0)\right),\left(x^{1}(1), x^{2}(1)\right), \ldots$, where $x^{1}(t)$ represents the first half of $t-t h$ year and $x^{2}(t)$ the second. Now model III can be written:

$$
\left.\begin{array}{rl}
B^{(1)} x^{1}(1) & \leqq \rho f^{1}(1)+A^{(2)} x^{2}(0) \\
B^{(2)} x^{2}(1)-A(1) x^{1}(1) & \leq \rho f^{2}(1) \\
B^{(1)} x^{1}(t+1)-A^{(1)} x^{2}(t) & \leq \rho^{t+1} f^{1}(t+1) \mid t \geq 1 \\
B^{(2)} x^{2}(t+1)-A^{(1)} x^{1}(t+1) & \leq \rho^{t+1} f^{2}(t+1)
\end{array}\right\}
$$

where, $A^{(1)}, A^{(2)}, B^{(1)}$ and $B^{(2)}$ are $m_{3} \times m_{3}$-matrices and $f^{\prime}(1), f^{2}(1), f^{1}(2), f^{2}(2), \ldots$ a sequence of $m_{3}$-dimensional vectors.

When we define the $\left(m_{3}+m_{3}\right) \times\left(n_{3}+n_{3}\right)$-matrices

$$
\underline{B}:=\left[\begin{array}{ccc}
B^{(1)} & , & 0 \\
-A & (1) & B
\end{array}\right], \quad \underline{A}:=\left[\begin{array}{ll}
0, & A^{(2)} \\
0, & 0
\end{array}\right],
$$

and the sequence of $\left(m_{3}+m_{3}\right)-d i m e n s i o n a l$ vectors

$$
\underline{f(t)}:=\left[\begin{array}{l}
f^{1}(t) \\
f^{2}(t)
\end{array}\right], \quad t \geq 1,
$$

then system $(1.5 .1)$ can be written

$$
\left.\begin{array}{rlrl}
B \times(1) & \leqq p \underline{E}(1)+A \times(0) & \\
B \times(t+1)-A x(t) & \leqq p^{t+1} \underline{E}(t+1), & t \geq 1 \\
\underline{x}(t) \geqq 0, & t \geqq 1
\end{array}\right\}
$$


where $x(0), \underline{x}(1), \ldots$ is a sequence of $\left(n_{3}+n_{3}\right)$-dimensional vectors which corresponds with a sequence $\left(x^{1}(0), x^{2}(0)\right),\left(x^{1}(1), x^{2}(1)\right), \ldots$ satisfying (1.5.1).

Let $\left(p^{1}(1) \cdot p^{2}(1)\right),\left(p^{1}(2), p^{2}(2)\right), \ldots$ be the vectors of corresponding objective functions $(1.4 .9)$ and $(1.4 .10)$. When we define

$$
\underline{p}(t):=\left[\begin{array}{l}
p^{1}(t) \\
p^{2}(t)
\end{array}\right], t \geq 1
$$

then the corresponding objective function for sequences $\underline{x}(1), \underline{x}(2), \ldots$ satisfying $(1.5 .4)$, can be written:

$$
\begin{aligned}
& \sum_{t=1}^{T} \pi^{t} \mathrm{P}(t)^{\prime} x(t), T \geq 1, \\
& \sum_{t=1}^{\infty} \pi^{t} \underline{p}(t)^{\prime} \times(t) .
\end{aligned}
$$

Thus, we find again that growth model IV, consisting of (1.5.4), (1.5.6), and $(1.5 .7)$, has the same form as the growth nodels I to III. Moreover, the definitions $(1.5 .2)$ and $(1.5 .5)$ and property $1.4-\mathrm{g}$ imply:

Each column vector E.j of matrix A which contains one or more negative components, corresponds with a non-negative column vector $\underline{b}_{. j}$ of matrix $\underline{B}$ and with non-positive components $\underline{p}_{j}(t)$ of the vectors $\underline{p}(1), \underline{p}(2), \ldots$.

1.6 The linear programming problem over an infinite horizon.

It appears that all growth models I to IV give rise to a linear programming problem consisting of the inequalities: 


$$
\left.\begin{array}{rlrl}
B \times(1) & \leqq \rho f(1)+A x(0) & \\
B x(t+1)-A x(t) & \leq \rho^{t+1} f(t+1), & t \geqq 1 \\
x(t) & \geqq 0, & t \geqq 1
\end{array}\right\} \quad(1.6 .1)
$$

and an objective function

$$
\sum_{t=1}^{\infty} \pi^{t} p(t)^{\prime} x(t),
$$

to be maximalized over the $n$-dimensional vectors $x(1), x(2), \ldots$, which satisfy'(1.6.1). Herein:

- A and $B$ are $m \times n-m a t r i c e s$.

- $f(1), f(2), \ldots$, is a sequence of m-dimensional vectors for which m-dimensional vectors $\underline{f}$ and $\bar{f}$ exist, such that:

$$
-\underline{f} \leq f(t) \leq F, \quad t \geq 1
$$

$-p(1), p(2), \ldots$, is a sequence of n-dimensional vectors for which $n$-dimensional vectors $p$ and $\bar{p}$ exist, such that:

$$
-\underline{p} \leqq p(t) \leqq \bar{p}, \quad t \geqq 1
$$

- $\rho$ and $\pi$ are positive coefficients.

- $x(0)$ is a given initial n-dimensional vector, which is nonnegative.

With respect to the matrices $A$ and $B$ and the sequences of vectors $f(1), f(2), \ldots$ and $p(1), p(2), \ldots$, it is found that, in these growth models, at least one of the following conditions is satisfied:

a) Each row vector $b_{i}$. of matrix $B$ which contains one or more negative components, corresponds with a non-negative row vector a $i$ of matrix $A$, and with non-negative components $f_{i}(t)$ of the vectors $f(1), f(2), \ldots$ :

b) Each column vector a.j of matrix A which contains one or more 
negative components, corresponds with a non-negative column vector $b_{. j}$ of matrix $B$, and with non-positive components $p_{j}(t)$ of the vectors.p(1), $p(2), \ldots$.

In this study we investigate a 1 inear programming problem which possesses the structure as sketched above. In this investigation another linear programming problem arises naturally. This problem consists of the linear inequalities:

$$
\left.\begin{array}{rl}
B^{\prime} u(t)-A^{\prime} u(t+1) & \geqq \pi^{t} p(t), t \geqq 1 \\
u(t) & \geqq 0, t \geqq 1
\end{array}\right\},
$$

and the objective function

$$
x(0)^{\prime} A^{\prime} u(1)+\sum_{t=1}^{\infty} \rho^{t} f(t) \cdot u(t),
$$

to be minimalized over the m-dimensional vectors $u(1), u(2), \ldots$, which satisfy $(1.6 .5)$.

The quantities appearing in this problem are the same as that of the first problem.

It will be shown later, that the coherence between both problems is of the same nature as the coherence between two linear programming problems over a finite horizon, which are dual with respect to each other. This offers the possibility () to interpret a sequence of vectors $u(1), u(2), \ldots$, which satisfies $(1.6 .4)$ and for which $(1.6 .5)$ attains its minimal value, as a sequence of prices, $i . e .: u_{i}(t)$ represents the price of the $i-t h$ goods at the moment of the period change $(t-1, t)$. That means that, in the context of growth models I to IV, the expression

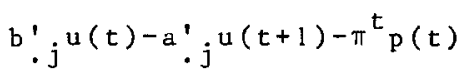

may be taken as the netto costs of the j-th process per unit of 
intensity in the t-th period. Thus, in this manner, we may interpret a sequence of vectors $u(1), u(2), \ldots$, which satisfies $(1.6 .4)$ as a sequence of prices such that in none of the periods any process yields netto benefits. The expression (1.6.6) might be taken as the value of the exogeneous goods $x(0)^{\prime} A^{\prime}+\rho f(1), p^{2} f(2), \rho^{3} f(3), \ldots$, at these prices.

A further interpretaion of the conditions a) and b) can be given as follows. It will be shown (3.2) that condition a) implies that all sequences $x(1), x(2), \ldots$, satisfying (1.6.1) for some initial vector $x(0)$, also satisfying:

$$
A x(t)+p^{t+1} f(t+1) \geq 0 \quad t \geq 1
$$

This means that for every period $t \geqq 1$ the expression $A x(t)+\rho^{t+1} f(t+1)$ may be interpreted as the quantity of goods available for the processes at the beginning of period $t+1$. In that respect we can say that in such an economy all goods are transferred from a preceeding period to the succeeding period. For that reason we call a system (1.6.1) directed, if it satisfies condition a).

In a similar way it appears that condition b) implies that all sequences $u(1) ; u(2), \ldots$ satisfying $(1.6 .5)$, also satisfy:

$$
B^{\prime} u(t)-\pi^{t} p(t) \geqq 0, \quad t \geqq 1
$$

Since, for all periods $t \geq 1$, the value of the expression $b^{\prime}, j u(t)-\pi^{t} p_{j}(t)$ is non-negative, and since the vector $u(t)$ may be taken as the prices of the goods at the beginning of period $t$, the expression $b^{\prime} \cdot j(t)-\pi{ }^{t} p_{j}(t)$ may be interpreted as the costs per unit of activity level for the $j-t h$ process in period t. Moreover, since $(1.6 .6)$ may be interpreted as the netto costs, one might say that the costs $b^{\prime}, j(t)-\pi^{t} p_{j}(t)$ are always account$a b l e$ at the end of period $t$. Therefore, we shall call a system (1.6.5) directed, if it satisfies condition b). 
With respect to the optimization aspect of the two linear programming problems described above, a sequence of n-dimensional vectors $\hat{x}(1), \hat{x}(2), \ldots$, will be called an optimal solution of the first problem if this sequence satisfies $(1.6 .1)$ and if no sequence of $n$-dimensional vectors $x(1), x(2), \ldots$ exists, which satisfies $(1.6 .1)$ as we 11 as

$$
\sum_{t=1}^{T} \pi^{t} p(t)^{\prime} x(t) \geqq \sum_{t=1}^{T} \pi^{t} p(t)^{\prime} \hat{x}(t)+\varepsilon, \quad T \geqq T^{*}
$$

for some positive number $\varepsilon$ and some period $T^{*} \geqq 1$.

In a similar manner, a sequence of m-dimensional vectors. û(1), $\hat{u}(2), \ldots$ will be called an optimal solution of the second problem if this sequence satisfies $(1.6 .5)$ and if no sequence of m-dimensional vectors $u(1), u(2), \ldots$ exists, which satisfies $(1.6 .5)$ as we 11 as

$x(0)^{\prime} A^{\prime} u(1)+\sum_{t=1}^{T} \rho^{t} f(t)^{\prime} u(t) \leq x(0)^{\prime} A^{\prime} \hat{u}(1)+\sum_{t=1}^{T} \rho^{t} f(t)^{\prime} \hat{u}(t)-\varepsilon, T \geq T^{*}$,

for some positive number $\varepsilon$ and some period $T^{\star} \geqq 1$.

The most important questions which are dealt with this study are the following:

- When do exist sequences of $n$-dimensional vectors $x(1), x(2), \ldots$ atisfying $(1.61)$. and when do exist sequences of m-dimensional vectors $u(1), u(2), \ldots$ satisfying (1.6.5)?

- When do optimal solutions exist for the first and the second problem?

- What is the symptotic behavior for $t \rightarrow \infty$ of optimal solutions $x(1), x(2), \ldots, x(t), \ldots$ and $u(1), u(2), \ldots, u(t), \ldots$ of the first and the second linear programing problem resp.? 


\subsection{Summary of the most important results.}

Presumed that at least one of the conditions $1.6-a$ or $1.6-b$ is satisfied, it appears that the linear programming problem consisting of $(1.6 .1),(1.6 .2)$ and of $(1.6 .5),(1.6 .6)$ are sensible only if $\rho \pi<1$. In that case, we found that, under certain conditions which are somewhat stronger than the assumption that both problems possess feasible solutions, the problems both possess optimal solutions:

It appears that the coherence between both problems is of a similar nature as the coherence between two linear programming problems in a finite dimensional space which are dual with respect to each other.

The most advanced results are obtained when the sequences of vectors $f(1), f(2), \ldots$ and $p(1), p(2), \ldots$, are supposed to be constant ever since some period $k \geqq 1$. Then, under some additional conditions, it can be shown that all optimal solutions $\hat{x}(1), \hat{x}(2), \ldots$ and $\hat{u}(1), \hat{u}(2), \ldots$ of the first and the second problem converge to certain fixed vectors $\tilde{x}$ and $\tilde{u}$ in the following manner:

$$
\begin{aligned}
& \lim _{t \rightarrow \infty}\left(\frac{1}{\rho}\right)^{t} \hat{x}(t)=\tilde{x}, \\
& \lim _{t \rightarrow \infty}\left(\frac{1}{\pi}\right) \hat{u}(t)=\tilde{u} .
\end{aligned}
$$

This property offers the possibility to construct a linear programing problem over a finite horizon from which all optimal solutions of the original infinite horizon can be found. 


\section{MATHEMATICAL FORMULATION OF THE LINEAR PROGRAMMING SYSTEM.}

\subsection{Introduction.}

First, we introduce a number of general concepts and notations. With the help of these implements the formulation of the linear programming system is given, which forms the central theme of this study. Finally, some concepts will be introduced with respect to the structure of this linear programming problem.

$2.21_{1}-$ and $1_{\infty}$-space.

The(real) 1, - and $11_{\infty}$-spaces are particular specimens of the $s 0$ called $l_{p}$-spaces $(5: 103)$. They are defined as follows.

The $1,-s p a c e$ is a vectorspace consisting of the sequences of (real) numbers $\left\{\xi_{i}\right\}_{1}^{\infty}$ for which

$$
\sum_{i=1}^{\infty}\left|\xi_{i}\right|<\infty .
$$

The norm of an element $x:=\left\{\xi_{i}\right\}_{1}^{\infty} \in 1_{1}$ is defined by

$$
\|x\|_{1}:=\sum_{i=1}^{\infty}\left|\xi_{i}\right|
$$

The $1_{\infty}$-space is a vectorspace consisting of the sequences of (real) numbers $\left\{\xi_{i}\right\}_{j}^{\infty}$ for which

$$
\sup _{i}\left|\xi_{i}\right|<\infty \text {. }
$$

The norm of an element $x:=\left\{\xi_{i}\right\}_{1}^{\infty} \in 1_{\infty}$ is defined by

$$
\|x\|_{\infty}:=\sup _{i}\left|\xi_{i}\right|
$$

The 1, - and 1 -norm may be introduced in an similar way for finite dimensional vector spaces. 
The $1_{1}$ - and $1_{\infty}$-spaces considered by us possess a special structure which can be described in the following manner.

Consider the sequences of vectors $\{x(t)\}_{1}^{\infty}$ in a $k$-dimensional real vector space $R^{k}$. The set of such sequences may be taken as an $\infty$-dimensional vector space and denoted by $1^{k}$. This leads to the following formal definition:

$$
1^{k}:=\left\{x:=\{x(t)\}_{1}^{\infty} \mid x(t) \in R^{k}, \quad t \geqq 1\right\} .
$$

Now we wish to introduce the $1_{1}^{k}$-space to be defined as the set of vectors in $1^{k}$ for which

$$
\sum_{t=1}^{\infty} \sum_{i=1}^{k}\left|x_{i}(t)\right|<\infty,
$$

with the norm:

$$
\|x\|_{1}:=\sum_{t=1}^{\infty} \sum_{i=1}^{k}\left|x_{i}(t)\right| .
$$

In a similar way we define the $1_{\infty}^{k}$-space as the set of vectors in $1^{\mathrm{k}}$ for which

$$
\sup _{t} \max _{i}\left|x_{i}(t)\right|<\infty .
$$

The norm of this space is defined by

$$
\|x\|_{\infty}:=\sup _{t} \max _{i}\left|x_{i}(t)\right| .
$$

It appears that every $1_{1}^{k}$ - or $l_{\infty}^{k}$-space may be considered as an $1_{1}$ - or $1_{\infty}$-space resp. For if $x:=\{x(t)\}_{1}^{\infty} \in 1_{1}^{k}$, then the sequence $\left\{\xi_{r}\right\}_{1}^{\infty}$ defined by

$$
\xi_{r=k(t-1)+i}:=x_{i}(t), \quad i=1,2, \ldots, k, \quad t \geqq 1,
$$

is a vector of $1_{1}$ with the same norm. The other way round, with the help of an opposite process every vector of $l_{1}$ may be identified with a vector of $1_{1}^{k}$ possessing the same norm. 
A similar relation may be constructed between $1_{\infty}$ and $1_{\infty}^{\mathrm{k}}$. This means that a11. properties of the $1_{1}$ - and $1_{\infty}-s p a c e$ simply can be transferred to the $1_{1}^{k}$ - and $1_{-\infty}^{k}$-spaces resp.

We shall also use the $1_{1}$ - and $1_{\infty}$-norm for vectors $x \in 1^{k}$ in another way, namely:

$$
\begin{aligned}
& \|x\|_{1}^{S, T}:=\sum_{t=S}^{T} \sum_{i=1}^{k}\left|x_{i}(t)\right|, \quad T \geq s \geq 1 . \\
& \|x\|_{1}^{S, T}:=\underset{T \leq t \leq S}{S} \underset{i}{\max }\left|x_{i}(t)\right|, \quad T \geq S \geq 1 .
\end{aligned}
$$

Finally, we define the positive cone of $1^{k}, 1_{1}^{k}$ and $1_{\infty}^{k}$ by:

$$
\begin{aligned}
& 1_{+}^{k}:=\left\{:\{x(t)\}_{1}^{\infty} \in 1^{k} \mid x(t) \geqq 0, \quad t \geq 1\right\}, \\
& I_{1+}^{k}:=1_{1}^{k} \cap 1_{+}^{k} \\
& 1_{\infty+}^{k}:=1_{\infty}^{k} \cap 1_{+}^{k}
\end{aligned}
$$

A well known property of the $1,-s p a c e$ is that the positive cone defined in this manner does not have an interior point. However, the interior of $1_{\infty+}^{k}$ is not empty and is defined by

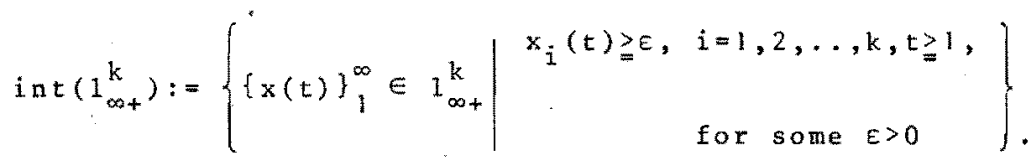

\subsection{The $\alpha-t r a n s$ form of $x \in 1^{k}$.}

It is easy to see that for every positive scalar $\alpha$, the expression

$$
x_{\alpha}:=\{\alpha x(t)\}_{1}^{\infty}
$$


represents a one-to-one mapping of $1^{k}$ onto itself. This transformation which will be used frequently, will be indicated by the term $\alpha$-transformation $(x)$.

The coëfficient of transformation $\alpha$ will ever be positive. The image $x_{\alpha}$ of $x \in 1^{k}$ generated by this transformation will be called the $\alpha$-transform of $x$.

In connection with this transformation we introduce the following concept: a vector $x \in 1^{k}$ is called $\alpha$-dominated if

$$
\mathrm{x}_{1 / \alpha} \in 1_{\infty}^{\mathrm{k}}
$$

This is equivalent with the condition that a positive number M exists, such that

$$
\|x(t)\|_{\infty} \leq \alpha{ }^{t} M, \quad t \geq 1
$$

So, in this prospect the sequence of numbers $\|x(t)\|_{\infty}, t \geq 1$ is dominated by $\alpha^{t} M, t \geq 1$.

\subsection{Linear functionals.}

With the help of a vector $y \in 1^{k}$, a sequence of numbers can be joined to every $x \in 1^{k}$ in the following manner:

$$
\langle y, x\rangle_{T}:=\sum_{t=1}^{T} y(t) ' x(t), \quad P \geqslant 1 .
$$

If such a sequence converges, the limit will be denoted by

$$
\langle y, x\rangle_{\infty}:=\underset{T \rightarrow \infty}{=} \underset{T \rightarrow}{i m}\langle y, x\rangle^{*}
$$

The expression $(2.4 .1)$ and $(2.4 .2)$ may be taken as linear functionals on $1^{k}$. It is well known that for every $x \in 1_{1}^{k}$ and $y \in 1_{\infty}^{k}$ the sequence $\left\{\langle y, x\rangle_{T}\right\}_{T=1}^{\infty}$ converges.

(*) In the context of this investigation confusion with the well known concept of $z$-transformation seems to be excluded. 
This implies that for every $y \in 1_{\infty}^{k},\langle y, x\rangle_{\infty}$ is a bounded (and.so a continuous) 1 inear functional on $1_{1}^{k}$ and in the same manner, that for every $y \in 1_{1},\langle y, x\rangle_{\infty}$ is a bounded linear functional on $1_{\infty}^{k}$.

\subsection{Formulation of the linear programming system.}

Now we shall give a formal definition of the growth model as described in \$1.2 to \$1.5. To this end we consider solutions $x \in 1_{+}^{n}$ of the system of linear inequalities

$$
\left.\begin{array}{ll}
B \times(1) & \leqq \rho f(1)+A x(0) \\
B \times(t+1)-A x(t) \leqq \rho^{t+1} f(t+1), t \geq 1
\end{array}\right\},
$$

where

- A and $B$ are $m \times n$-matrices,

$-E:=\{f(t)\}_{1}^{\infty} \in 1_{\infty}^{\mathrm{m}}$,

- $x(0) \in \mathrm{R}^{\mathrm{n}}$ is the initial vector, always supposed to be non-negative,

- p is a positive scalar.

In connection with the economical back ground we shall term the numbers $0,1,2, \ldots$ used in the context of $(2.5 .1)$ as periods. Unless indicated otherwise, the initial vector $x(0)$ is supposed to be a fixed given quantity.

By introduction of so called slack variables $y:=\{y(t)\}_{1}^{\infty} \in 1_{+}^{m}$, the system (2.5.1) can be converted into the system of linear equalities:

$$
\left.\begin{array}{l}
B \times(1)+y(1)=\rho f(1)+A \times(0) \\
B \times(t+1)-A \times(t)+y(t+1)=\rho^{t+1} E(t+1), \quad t \geq 1
\end{array}\right\} .
$$

The systems $(2.5 .1)$ and $(2.5 .2)$ are equivalent and from now on, will be treated without distinction. 
Now we shal1 consider for every $x \in 1_{+}^{n}$ the linear functionals

$$
\left\langle\mathrm{p}_{\pi}, x\right\rangle_{T}:=\sum_{t=1}^{T} \pi^{t} p(t) \cdot x(t), \quad T \geqslant 1,
$$

where $p \in 1_{\infty}^{n}$ and $\pi$ is a positive scalar.

System (2.5.1) or $(2.5 .2)$, together with the linear functionals (2.5.3) will be analysed simultaneously with another system already mentioned in chapter 1. In mathematical respect these systems are related by a so called duality-relation. This relation will be pointed out later and in the first instance illustrated in $\$ 2.7$. Adopting the common nomenclature of the theory of linear programing in a finite dimensional space, we shall call the system (2.5.1) or (2.5.2) together with the linear functionals (2.5.3) the primal system and the system to be formulated now, the dual system. The whole consisting of the primal and dual system will be indicated by the term linear programing system or LP-system In the dual system we consider vectors $u \in 1_{+}^{m}$ satisfying

$$
B^{\prime} u(t)-A^{\prime} u(t+1) \geq \pi^{t} p(t), \quad t \geq 1
$$

or formulated as a system of linear equalities, we consider vectors $(u, v) \in 1_{+}^{m} \times 1_{+}^{n}$ satisfying.

$$
B^{\prime} u(t)-A^{\prime} u(t+1)-v(t)=\pi^{t} p(t), \quad t \geq 1,
$$

where $A^{\prime}$ and $B^{\prime}$ are the transposed matrices of (2.5.1), while $p:=\{p(t)\}_{1}^{\infty}$ and $\pi>0$ correspond with $(2.5 .3)$.

With a fixed initial vector $x(0)$ we further consider for all vectors $u \in 1_{+}^{m}$ or $(u, v) \in 1_{+}^{m} \times 1_{+}^{n}$ satisfying $(2.5 .4),(2.5 .5)$ resp. the linear functionals.

$$
x(0)^{\prime} A^{\prime} u(1)+\sum_{t=1}^{T} \rho^{t} f(t)^{\prime} u(t), \quad T \geqq 1,
$$


where $f:=\{f(t)\}_{1}^{\infty} \in 1_{\infty}^{m}$ and $\rho>0$ are quantities already introduced in the primal system.

Denoting the sequence of vectors $\left\{f(1)+\frac{1}{\rho} A x(0), f(2), \ldots, f(t), \ldots\right\}$ by $f^{\circ}:=\left\{f^{\circ}(t)\right\}_{1}^{\infty}$, the linear functionals of $(2.5 .6)$ can be written

$$
\left\langle f_{\rho}^{o}, u\right\rangle_{T}:=\sum_{t=1}^{T} \rho^{t} f^{0}(t) \cdot u(t), \quad T \gg 1 .
$$

The systems $(2.5 .4)$ or $(2.5 .6)$, combined with $(2.5 .7)$, form the dual system.

We wish to introduce some terms frequently appearing in this investigation.

The LP-system is called:

- primal feasible (P-feasible) when system (2.5.1) possesses a solution $x \in 1_{+}^{n}$; this solution $x$ or a solution $(x, y) \in 1_{+}^{n} \times 1_{+}^{m}$. of (2.5.2) will be called primal feasible,

- virtuallyprimal feasible $\left(\mathrm{P}^{\circ}\right.$-feasible) when there is a initial vector $x(0)$ such that the LP-system is P-feasible,

- dual-feasible (D-feasible) when system (2.5.4) possesses a solution $u \in 1_{+}^{m}$; this solution $u$ or a solution $(u, v) \in 1_{+}^{m} \times 1_{+}^{n}$ of (2.5.5) will be called dual feasible,

- feasible (P- and D-feasible) when the LP-system is P- and Dfeasible,

- virtuallyfeasible $\left(P^{\circ}\right.$ - and $D$-feasible) when the LP-system is

The following terms have reference to the existence of special sort of feasible solutions:

The LP-system is called:

- primal regular (P-regular) when (2.5.2) possesses a solution $(x, y) \in 1_{+}^{n} \times 1_{+}^{m}$ such that $\left(x_{1 / 0}, y_{1 / 0}\right) \in 1_{\infty+}^{n} \times$ int $\left(1_{\infty+\infty}^{m}\right)$; this solution is called primal regular.

- dual regular (D-regular) when (2.5.5) poccesses a solution 
$(u, v) \in 1_{+}^{m} \times 1_{+}^{n}$ such that $\left(u_{1 / \pi}, v_{1 / \pi}\right) \in 1_{\infty+}^{m} \times \operatorname{int}\left(1_{\infty+}^{n}\right) ;$ this solution is called dual-regular.

The notions virtually primal regular $\left(P^{\circ}\right.$-regular), regular and virtuallyregular may be introduced in a similar manner as the corresponding notions with respect to the feasibility.

We further shall call the LP-system (virtually) superregular if simultaneously:

$-p \pi<1$,

- the LP-system is (virtually) regular,

- the systems

$$
\left.\begin{array}{ll}
B x(t+1)-A x(t)+y(t+1)=\left(\frac{1}{\pi}\right)^{t} f(t), & t \geqq R \\
B^{\prime} u(t)-A^{\prime} u(t+1)-v(t)=\left(\frac{1}{p}\right)^{t} p(t), & t \geqq R
\end{array}\right\},
$$

have solutions $(x, y)$ and $(u, v)$ for some $k \geqq 1$, such that $\left(x_{\pi}, y_{\pi}\right) \in 1_{\infty+}^{n}+x \operatorname{int}\left(1_{\infty+}^{m}\right)$ and $\left(u_{p}, v_{\rho}\right) \in 1_{\infty+}^{m} x \operatorname{int}\left(1_{\infty+}^{n}\right)$.

The systems $(2.5 .1)$ and $(2.5 .2)$ will be called primal feasibilityconditions and the systems $(2.5 .4)$ and (2.5.5) dual feasibility conditions.

The linear functionals (2.5.3) and (2.5.7) will be indicated by the terms primal and dual objective functions resp.

on the set of primal and dual feasible solutions we wish to install a partial ordering which refers directly to the optimization aspect of the LP-systems as mentioned in $\$ 1.2$ and $\$ 1.6$.

To this end a P-feasible solution $x$ is called inferior with respect to a p-feasible solution $\bar{x}$, if a number $\varepsilon>0$ and a period $s \geqq 1$ exist such that

$$
\left\langle\mathrm{p}_{\pi}, \mathrm{x}\right\rangle_{\mathrm{T}} \leqq\left\langle\mathrm{p}_{\pi}, \overline{\mathrm{x}}\right\rangle_{\mathrm{T}}-\varepsilon, \quad \mathrm{T} \geqq S
$$


A D-feasible solution $u$ is called inferior with respect to a Dfeasible solution $\bar{u}$, if a number $\varepsilon>0$ and a period $s \gg l$ exist such that

$$
\left\langle\mathrm{f}_{\rho}^{\circ}, \mathrm{u}\right\rangle_{\mathrm{T}} \geq\left\langle\mathrm{f}_{\rho}^{\circ}, \overline{\mathrm{u}}\right\rangle_{\mathrm{T}}+\varepsilon, \quad \mathrm{T} \geqslant \mathrm{S} .
$$

\subsection{Formulation of the problem.}

Briefly summarized this investigation deals with the following problems:

a) When is the IP-system feasible?

b) Are the sets of $P$ - and D-feasible solutions bounded in some respect?

c) Does an upper bound exist for the primal partial objective function and a lower bound for the dual partial objective function?

d) When do 2 P-feasible $\bar{x}$ and $p$-feasible $\bar{u}$ exist, such that the sequences $\left\{\left\langle p_{\pi}, \bar{x}\right\rangle_{T}\right\}_{T=1}^{\infty}$ and $\left\{\left\langle p_{T}, \bar{u}\right\rangle_{T}\right\}_{T=1}^{\infty}$ converge and such that every $P$-feasible $x$ and every $D-f e a s i b l e ~ w$, for which the sequences $\left\{\left\langle p_{T}, x\right\rangle_{T}\right\}_{T=1}^{\infty}$ and $\left\{\left\langle f_{\rho}^{o}, u\right\rangle_{T}\right\}_{T=1}^{\infty}$ are not convergent, is inferior with respect to $\bar{x}, \bar{u}$ resp.?

It appears that $P$ - and D-feasible solutions $\bar{x}, \vec{u}$ as mentioned under d) exists, when the LP-system is regular and $p \pi<1$. With regard to the optimalization aspect of the LP-system, we can conclude that these feasible solution $\bar{x}, \bar{u}$ are "better" then those for which the sequences of partial objective functions do not converge. For this reason we further restrict ourselves to the subset of $\mathrm{P}$ - and $\mathrm{D}$-feasible solutions, for which the sequence of partial objective functions converge, and direct the investigation to the following problems:

e) When does a P-feasible $\hat{x}$ exists, for which the linear functional $\left\langle p_{\pi}, x\right\rangle_{\infty}$ on this subset of $P$-feasible solutions attains his supremum? Every p-feasible solution $\hat{x}$ with this property will be called a p-optimal solution.

f) When does a D-feasible â exists, for which the linear functional $\left\langle f_{\rho}^{0}, u\right\rangle_{\infty}$, on the subset of D-feasible solutions as discribed 
above, attains his infinum? Every D-feasible solution $\widehat{u}$ with this property will be called a D-optimal solution.

g) What is the asymptotic behavior of the components $\hat{x}(t)$ and $\hat{u}(t)$ of $P-$ and $D$-optimal solutions $\hat{x}$ and $\hat{u}$ for $t \rightarrow \infty$ ?

We write the problems posed in e) and f) as follows

$$
\begin{aligned}
& \begin{array}{l|l}
\sup \left\langle p_{\pi}, x\right\rangle_{\infty} & \leq \rho f^{0}(1) \\
x \in 1_{+} & B x(t+1)-A x(t) \leqq \rho^{t+1} f^{0}(t+1), t \geq 1
\end{array} \quad(2 \cdot 6 \cdot 1) \\
& \underset{u \in 1_{+}}{\inf }\left\langle f_{p}^{o}, u\right\rangle_{\infty} \mid B^{\prime} u(t)-A^{\prime} u(t+1) \geq \pi^{t} p(t), \quad t \geq 1
\end{aligned}
$$

The problems $(2.6 .1)$ and $(2.6 .2)$ will be called the primal and dual problem resp. and the whole, consisting of $(2.6 .1)$ and $(2.6 .2)$ the linear programming problem (LP-problem).

We dominate the functionals $\left\langle p_{\pi}, x\right\rangle_{\infty}$ and $\left\langle f_{p}^{0}, u\right\rangle_{\infty}$ as the primal and dual objective function resp.

\subsection{Duality.}

We illustrate the duality relation between the primal and dual problem already suggested with the help of a LP-problem formulated for finite horizon problem.

To this end, we consider the following programming problem in a finite dimensional euclidean space:

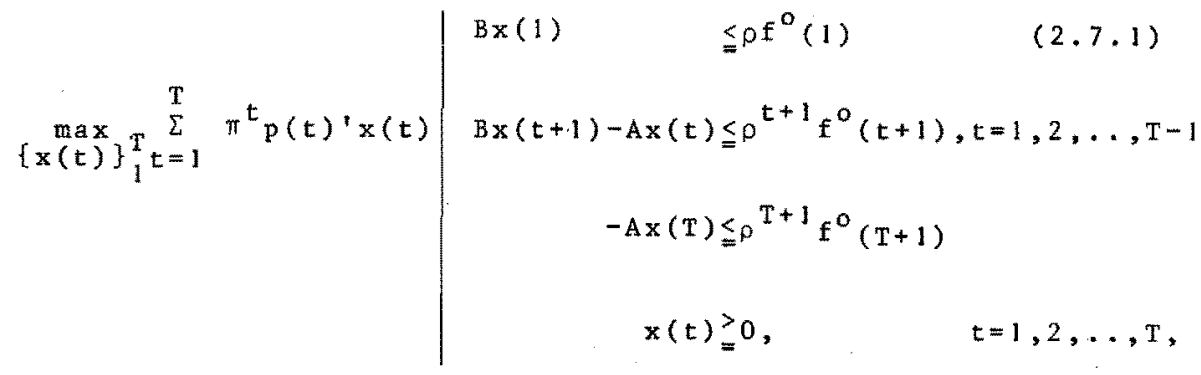


where all quantities are the same as used in $\$ 2.5$.

This problem can be taken as being generated by the cutting down of the primal problem $(2.6 .1)$ at a period $T$.

Applying the well known duality rules from the theory of linear programing in a finite dimensional space on (2.7.1) we encounter the following problem:

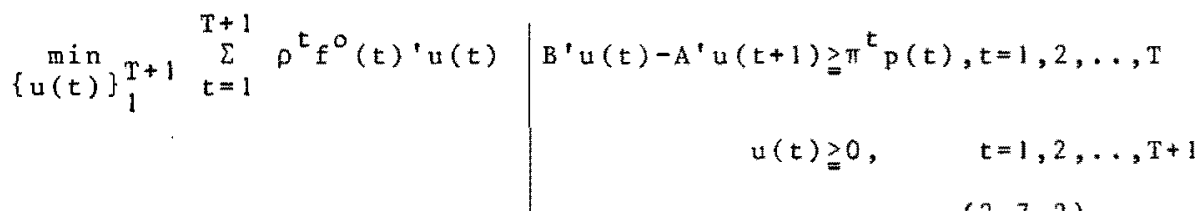

This problem, too, may be considered as being generated by the cutting down of the dual problem (2.6.2).

The mathematical coherence between the primal and dual problem of $\$ 2.6$ will appear to be of the same nature as that between the problems sketched above.

In this context, we remark that the dual system can be written in a similar form as the primal system:

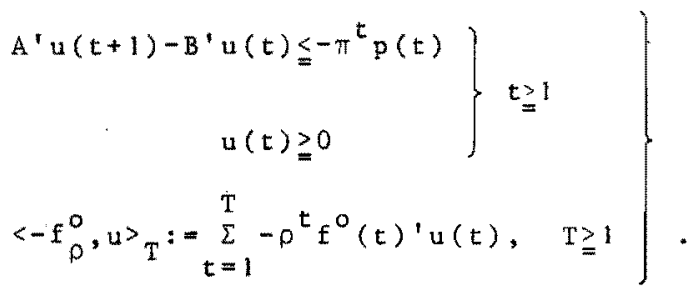

Consequently the dual problem can be written:

$$
\underset{u \in 1_{+}}{s u p}\left\langle-f_{\rho}^{o}, u\right\rangle_{\infty} \mid A^{\prime} u(t+1)-B^{\prime} u(t) \leq-\pi^{t} p(t), \quad t \geqq 1 .
$$

By virtue of this similarity between the primal and dual system (problem), further to be indicated by the term symmetry, many properties of the primal system (problem) may simply be transferred 
to the dual system (problem).

Finally, we point out a difference which is caused by the appearence of the initial vector $x(0)$ in the primal system (problem).

This difference has led to the introduction of two concepts of feasibility. for the primal system, whereas one type of feasibility suffices for the dual system.

\section{8 a-transformed primal and dual systems.}

The $\alpha$-transformation (52.3) of $1^{k}$ onto itself suggests a similar transformation for the primal and dual systems, which we want to introduce now.

We shall call the system

$$
\left.\begin{array}{rl}
B x(1) & \leqq \alpha \rho f^{\circ}(1) \\
B x(t+1)-\alpha A x(t) \leqq(\alpha \rho)^{t+1} f^{\circ}(t+1), \quad t \geq 1 \\
\left.<p_{\pi / \alpha}, x\right\rangle_{T}:=\sum_{t=1}^{T}\left(\frac{\pi}{\alpha}\right)^{t} p(t)^{\cdot} x(t), T \geq 1
\end{array}\right\},
$$

The $\alpha$-transformed primal system, and

$$
\left.\begin{array}{rl}
B^{\prime} u(t)-\frac{1}{\alpha} A^{\prime} u(t+1) & \geq(\alpha \pi) t p(t), \quad t \geq 1 \\
\left\langle f_{\rho}^{0} / \alpha, u\right\rangle_{T}: & =\sum_{t=1}^{T}\left(\frac{Q}{\alpha}\right)^{t} f^{\circ}(t) \cdot u(t), \quad T \geqslant 1
\end{array}\right\}
$$

\section{the $\alpha-t r a n s f o r m e d$ dual system.}

Clearly, $x$ is a P-feasible solution (of the untransformed LP-system) if and only if $x_{\alpha}$ satisfies $(2,8,1)$, and $u$ is a D-feasible solution (of the untransformed LP-system) if and only if ${ }_{\alpha}$ satisfies (2.8.2) In the same manner, we shall call 
\begin{tabular}{ll|l}
$\sup _{x \in I_{+}}\left\langle p_{\pi / \alpha}, x>T\right.$ & $\leqq(\alpha \rho) f^{o}(1)$ \\
$B x(1)$ & $B x(t+1)-\alpha A x(t) \leqq(\alpha \rho)^{t+1} f^{0}(t+1), t \geq 1$.
\end{tabular}

The $\alpha$-transformed primal problem, and

$\underset{u \in 1_{+}^{m}}{\inf }\left\langle f_{\rho}^{0}, u\right\rangle_{\infty} \mid B^{\prime} u(t)-\frac{1}{\alpha} A^{\prime} u(t+1) \geqq(\alpha \pi)^{t} p(t), \quad t \geq 1$,

the $\alpha$-transformed dual-problem.

A specific property of these problems is:

$x$ is a p-optimal solution(of the untransformed LP-problem) if and only if $x_{\sigma}$ is a p-optimal solution of the $\alpha$-transformed primal problem. A similar relation holds for the dual problem.

2.9 Concepts with respect to the structure of the LP-system.

We shall analyse the LP-system under different suppositions.

The most important of which are the following:

The LP-system is called:

- exponential, when

$$
\left.\begin{array}{l}
f(t)=q \\
p(t)=p
\end{array}\right\} t \geq 1
$$

- semi-exponential, when

$$
\left.\begin{array}{l}
f(t)=\tilde{f} \\
p(t)=\tilde{p}
\end{array}\right\} t \geq T, \text { for some } T \geq 1 \text {, }
$$

i.e. if a period $T \gg l$ exists such that the sequences $\{f(t)\}_{T}^{\infty}$ and $\{\mathrm{p}(\mathrm{t})\}_{\mathrm{T}}^{\infty}$ are constant,

- primal directed (P-directed), if every row vector $b_{j}$, of $B$, which possesses a negative component, corresponds with a non- 
negative row vector $a_{i}$. of $A$, and with non-negative components $f_{i}(t), t \geqq l$ of the sequence $\{f(t)\}_{1}^{\infty}$. (see also $\$ 1$. )

- dual directed (D-directed), if every column a,jof A, which possesses a negarive component, corresponds with a non-negative column vector $b_{j_{j}}$ of $B$, and with non-positive components $p_{j}(t)$, $t \geq 1$ of the sequence $\{p(t)\}_{1}^{\infty}$.

We remark that the latter two definitions are symmetric; for witing the dual feasibility conditions as follows

$$
A^{\prime} u(t+1)-B^{\prime} u(t) \leqq \pi^{t}(-p(t))
$$

it appears that they correspond completely.

Finally, with the help of two examples, we introduce a brief notation for suppositions about the LP-system(problems):

- LP-system(P- or D-directed; $p(t) \rightarrow \tilde{p}, t \rightarrow \infty):$ the system is supposed to be $P-$ or $D-d i r e c t e d$ and the sequence $\{p(t)\}_{1}^{\infty}$ to converge to $\tilde{\mathrm{p}} \in \mathrm{R}^{\mathrm{n}}$.

- LP-problem $\left(\left(f^{\circ}, p\right) \in F^{\circ} \times P ; P-o r\right.$ D-directed $): f^{\circ}$ and $p$ are supposed to be vectors of $F^{\circ}, P$ resp. and such that, for these $\mathrm{F}^{\mathrm{O}}$ and $\mathrm{p}$, the LP-problem is $\mathrm{P}$ - or $\mathrm{D}$-directed. 


\section{DIRECTEDNESS, FEASIBILITY, AND REGULARITY.}

\subsection{Introduction.}

In this chapter a number of conditions will be derived with respe to the feasibility, regularity and boundness of feasible solutions In these derivations the concept of directedness takes a central place.

\subsection{Theorem.}

The L.P.-system is then and only then P-directed if for every $f(t), t \geq 1:$

each $(x, y, z) \in R_{+}^{n+n+m}$ satisfying

$$
B x-A y-z \leqq f(t) \text {, }
$$

also satisties

$$
-A y-z \leqq f(t) .
$$

Proof.

Necessary: suppose that for some $t \geq 1$, there is $a(x, y, z) \in R^{n+n}$ such that

$$
\left.\begin{array}{rl}
B x-A y-z & \leqq f(t) \\
-A y-z & \notin f(t)
\end{array}\right\} .
$$

Then an index $i$ exists for which $b_{i} 0$ and for which $a_{i}$. 0 or $f_{i}(r)<0$. This, however, is impossible in connection with the definition of P-directedness.

Sufficient: assume that the LP-system is not p-directed, then the is $a b_{i j}<0$ such that $a_{i} .0$ or $f_{i}(t)<0$ for some $t \geqq 1$. This implies that there exists $a(x, y) \in R_{+}^{\text {n }}$ such that for some $t \geqq 1$ and row-index i: 


$$
\left.\begin{array}{r}
b_{i,} x-a_{i} y \leqq f_{i}(t) \\
-a_{i} y>f_{i}(t)
\end{array}\right\} .
$$

From this it appears that it is possible to choose a $z \in \mathbb{R}_{+}^{\mathrm{m}}$ : $z_{i}=0$ and sufficiently large the other components, such that $(x, y, z)$ satisfies $(3.2 .1)$ but not $(3.2 .2)$.

\subsection{Theorem.}

The LP-system is then and only then D-directed if for every $p(t), t \geq 1:$

each $(u, v, w) \in R_{+}^{m+m+n}$ satisfying

$$
B^{\prime} u-A^{\prime} v+w \geq P(t)
$$

also satisfies

$$
B^{\prime} u+W \geq p(t)
$$

Proof.

The theorem follows from theorem 3.2 and from the symmetry between the primal and dual system.

\subsection{Remark.}

The following two propositions only will be used as auxiliary theorems.

\subsection{Proposition.}

Let $\Lambda$ be a diagonal $n \times n$-matrix which is defined for a LP-system (P- or D-directed) as follows:

- in case the LP-system is P-directed:

$$
\Lambda:=I \text {, }
$$

- in case the LP-system is not P-directed: 


$$
\left.\begin{array}{lll}
\lambda_{j j}:=1, & \text { if } & a_{j} \not \geq 0 \\
\lambda_{j j}:=0, & \text { if } & a, j \geq 0
\end{array}\right\},
$$

then for every $(x, y, z) \in 1_{+}^{n} \times 1_{+}^{m} \times 1_{+}^{m}$ satisfying

$$
B x(t)-A x(t-1)+y(t)=\gamma^{t} f(t)+z(t), t \geqq K,
$$

for some $\gamma>0, K \geq 1$, and for every monotonous non-increasing sequence of numbers $\{\theta(t)\}_{k-1}^{\infty}$, there exists a $\left(\{x(t)\}_{K-1}^{\infty},\{\mathrm{z}(t)\}_{K}^{\infty}\right) \in 1^{n} \times 1^{\text {m with }} x(K-1)=\theta(K-1) \times(K-1)$, such that

$$
\left.\begin{array}{rl}
B x(t)-A x(t-1)+y(t) & =\theta(t-1)\left(\gamma^{t} f(t)+z(t)\right) \\
X(t) & =\theta(t) \Lambda x(t)+\theta(t-1)(I-\Lambda) x(t) \\
y(t) & =\theta(t) y(t)
\end{array}\right\} .
$$

\section{Proof.}

First consider the case that the LP-system is P-directed, so that $\Lambda:=I$.

From theorem 3.3 it then follows that every $(x, y, z) \in 1_{+}^{n} \times 1_{+}^{\mathrm{m}} \times 1_{+}^{\mathrm{m}}$ satisfying (3.5.1) for some $Y>0, K \geqq 1$, also satisfies

$$
-A x(t-1) \leqq \gamma^{t} f(t)+z(t), t \geqq K
$$

Since the sequence $[\theta(t)]_{K-1}^{\infty}$ is monotonous non-increasing, this implies that

$$
-(\theta(t-1)-\theta(t)) A x(t-1) \leqq(\theta(t-1)-\theta(t))\left(\gamma^{t} f(t)+z(t)\right), t \geqq k .
$$

The equalities $(3.5 .1)$ imply:

$$
\theta(t)(B x(t)-A x(t-1)+y(t))=\theta(t)\left(\gamma^{t} f(t)+z(t)\right), t \geqq k \cdot(3 \cdot 5 \cdot 6)
$$


By adding (3.5.5) and $(3.5 .6)$ we find

$$
B \theta(t) x(t)-A \theta(t-1) x(t-1)+\theta(t) y(t) \leq \theta(t-1)\left(\gamma^{t} f(t)+z(t)\right), t \geqq K .
$$

Since $\Lambda:=$ I we may conclude that there exists a $(x, y) \in 1^{\mathfrak{n}} \times 1^{\text {m }}$ satisfying $(3.5 .2)$.

Now we consider the case that the LP-system is not P-directed. Then the D-directedness and the definition of $\Lambda$ imply that

$$
\left.\begin{array}{l}
B \geqq 0 \\
A(I-A) \geqq 0
\end{array}\right\} .
$$

Since $\{\theta(t)\}_{\mathrm{K}-1}^{\infty}$ is monotonous non-increasing and $\{\mathrm{x}(\mathrm{t})\}_{\mathrm{K}-1}^{\infty}$ is non-negative, we may conclude that

$$
\left.\begin{array}{l}
B \Lambda(\theta(t)-\theta(t-1)) x(t) \leqq 0 \\
-A(1-\Lambda)(\theta(t-1)-\theta(t)) x(t) \leq 0
\end{array}\right\} t \geqq R
$$

The equality (3.5.1) implies

$$
\theta(t-1)(B x(t)-A x(t-1)+y(t))=\theta(t-1)\left(\gamma^{t} f(t)+z(t)\right), t \geqslant k .
$$

Adding (3.5.8) and (3.5.9) it appears that there exists a $\left(\{x(t)\}_{K-1}^{\infty},\{\mathrm{z}(t)\}_{\mathrm{K}}^{\infty}\right)$ satisfying $(3.5 .2)$.

\subsection{Proposition.}

If the LP-system is $P$ - or D-directed, then for every $(x, y) \in I_{+}^{n} \times 1_{+}^{m}$ satisfying

$$
B x(t)-A x(t-1)+y(t)=\gamma^{t} f(t), t \geqq K,
$$

for some $Y>0, K \geqq 1$, and for every $\alpha>0, I>K$, a $(\bar{x}, \bar{y}) \in R_{+}^{n+m}$ exists such that 


$$
\left.\begin{array}{rl}
\left(B-\frac{1}{\alpha} A\right) \bar{x}+\bar{y} & =\sum_{t=K}^{T}(\gamma / \alpha)^{t} f(t)+\frac{1}{\alpha} A x(K-1) \\
\bar{x} & \geqslant \sum_{t=K}^{T-1}\left(\frac{1}{\alpha}\right)^{t} x(t) \\
\bar{y} & \geq \sum_{t=K}^{T-1}\left(\frac{1}{\alpha}\right)^{t} y(t)
\end{array}\right\}
$$

\section{Proof.}

Let $(x, y) \in 1_{+}^{n} \times 1_{+}^{m}$ be a solution of (3.6.1) for some $\gamma>0$, $\mathrm{K} \geqq 1$. Defining the sequence $\{\theta(t)\}_{K-1}^{\infty}$ for some $T>K$ by

$$
\left.\begin{array}{l}
\theta(t):=1, \quad t=K-1, K, \ldots, T-1 \\
\theta(t):=0, t \geq T
\end{array}\right\},
$$

it follows from proposition 3.5 that a $\left(\{x(t)\}_{K}^{\infty},\{y(t)\}_{K}^{\infty}\right)$ exists such that

$$
\begin{aligned}
& B_{X}(K)+\chi(K)=\gamma_{f(K)}^{K}+A x(K-1) \\
& B \times(t+1)-A x(t)+Z(t+1)=\gamma^{t+1} f(t+1), t=K, K+1, \ldots T-1 \\
& -A x(T) \leqq 0 \\
& \mathrm{x}(\mathrm{t})=\mathrm{x}(\mathrm{t}), \quad \mathrm{t}=\mathrm{k}, \mathrm{K}+1, \ldots \mathrm{T}-1 \\
& y(t) \geqq y(t) \quad t=K, K+1, \ldots T-1 \\
& x(T), y(T) \geqq 0
\end{aligned}
$$

From (3.6.3) the following inequalities may be derived 


$$
\left.\begin{array}{rl}
\left(B-\frac{1}{\alpha} A\right) \sum_{t=K}^{T}\left(\frac{1}{\alpha}\right)^{t} X(t)+\sum_{t=K}^{T}\left(\frac{1}{\alpha}\right)^{t} Z(t) & \leqq \sum_{t=K}^{T}\left(\frac{Y}{\alpha}\right)^{t} f(t)+\frac{1}{\alpha} A x(K-1) \\
\sum_{t=K}^{T}\left(\frac{1}{\alpha}\right)^{t} X(t) & \geqq \sum_{t=K}^{T-1}\left(\frac{1}{\alpha}\right)^{t} x(t) \\
\sum_{t=1}^{T}\left(\frac{1}{\alpha}\right)^{t} X(t) & \geqslant \sum_{t=1}^{T-1}\left(\frac{1}{\alpha}\right)^{t} y(t)
\end{array}\right\}
$$

From this the proposition follows immediately.

\subsection{Remark.}

The manner in which the supposition that the LP-system is $P$ - or D-directed functions appears especially from proposition 3.6. By this it is possible to cut down the LP-system to a system with a finite number of periods, which leads to a finite number of linear inequalities, as constructed in (3.6.2). The latter is essential to the argumentation of the next theorem.

\subsection{Theorem.}

For a LP-system (P- or D-directed; $f(t) \rightarrow \tilde{f}, t \rightarrow \infty)$ the following properties hold:

a) If the LP-system is $\mathrm{P}^{\circ}$-feasible, then the system

$$
\left.\begin{array}{rl}
\left(B-\frac{1}{\rho} A\right) z & \leqq \\
z & \geq 0
\end{array}\right\},
$$

has a solution.

b) If for some initial vector the LP-system has a p-feasible solution $(x, y)$ such that $y(t) \geqq \rho^{t} \bar{y}$ for some $\bar{y} \in$ int $\left(R_{+}^{m}\right)$ then the system

$$
\left.\begin{array}{rl}
\left(B-\frac{1}{\rho} A\right) z & <z \\
z & \geq 0
\end{array}\right\},
$$


has a solution.

c) If for some $k \geqslant 1$ and $\bar{y} \in$ int $\left(\mathrm{R}_{+}^{\mathrm{m}}\right)$ the system

$$
\left.\begin{array}{rl}
B x(t)-A x(t-1)+y(t) & =\left(\frac{1}{\pi}\right)^{t} f(t) \\
x(t-1) & \geqq 0 \\
y(t) & \geqq\left(\frac{1}{\pi}\right)^{t} \bar{y}
\end{array}\right\} t \geqq K,
$$

has a solution, then the system

$$
(B-\pi A) z<\nsucceq
$$

has a solution.

\section{Proof.}

(a) Let $(x, y) \in 1^{n+m}$ be a p-feasible solution for some $x(0)$. Then it follows from proposition 3.6 that the system

$$
\begin{aligned}
\left(B-\frac{1}{\rho} A\right) \bar{x}+\bar{y} & =\frac{1}{T}\left(\frac{1}{\rho} A x(0)+\sum_{t=1}^{T} f(t)\right) \\
\bar{x} & \geqq \frac{1}{T} \sum_{t=1}^{T}\left(\frac{1}{\rho}\right)^{t} x(t) \\
\bar{y} & \geqq \frac{1}{T} \sum_{t=1}^{T-1}\left(\frac{1}{\rho}\right)^{t} y(t)
\end{aligned}
$$

has a solution for every $T>1$.

Defining

$$
\begin{aligned}
& C:=\left\{z=\left(B-\frac{1}{\rho} A\right) \bar{x}+\bar{y} \mid \bar{x} \geq 0, \bar{y} \geq 0\right\}, \\
& g(T):=\frac{1}{T}\left(\frac{1}{\rho} A x(0)+\sum_{t=1}^{T} f(t)\right), \quad T>1,
\end{aligned}
$$

system (3.8.4) implies that 


$$
\mathrm{g}(\mathrm{T}) \in \mathrm{C}, \mathrm{T}>1
$$

The supposition $f(t) \rightarrow \breve{f}, \quad t \rightarrow \infty$ implies

$$
g(T) \rightarrow f, \quad t+\infty .
$$

From the definition of $C$ it follows (, 174) that this set is closed. On this ground, $(3.8 .7)$ and $(3.8 .8)$ imply $\tilde{f} \in C$.

In connection with the definition $(3.8,5)$ of $C$ we may conclude that the system (3.8.1) has a solution.

(b) Let $(x, y) \in 1^{n+m}$ be a p-feasible solution for some $x(0)$ such that

$$
y(t) \geqq p^{t} \bar{y}, \quad t \geq 1
$$

for some $\bar{y} \in \operatorname{int}\left(R_{+}^{\mathrm{m}}\right)$.

From $(3.8 .4),(3.8 .5),(3.8 .6)$ and $(3.8 .9)$ it may be derived that

$$
\left.\left(g(T)-\frac{1}{2} \bar{y}\right)\right) \in C, \quad T>1 \text {. }
$$

Then the convergence $(3.8 .8)$ implies $\left(\tilde{f}-\frac{1}{2} \bar{y}\right) \in C$ and there by the existence of a solution for (3.8.2)

(c) From proposition 3.6 it may be derived that the system

$$
\left.\begin{array}{rl}
(B-\pi A) \tilde{x}+\tilde{y} & =\frac{i}{T-K}\left(\pi A x(K-1)+\sum_{t=K}^{T} f(t)\right) \\
\tilde{x} & \geq 0 \\
y & \geq \frac{1}{2} \bar{y}
\end{array}\right\}
$$

has a solution for every $T>K$. The proof may be derived in a similar way as the one for b). 


\subsection{Theorem.}

For a LP-system (P- or D-directed; $p(t) \rightarrow \stackrel{q}{p}, t \rightarrow \infty)$ the following properties hold:

a) If the LP-system is D-feasible, then the system

$$
\left.\begin{array}{rl}
\left(B^{\prime}-\pi A^{\prime}\right) w & \geq \stackrel{2}{p} \\
w & \geq 0
\end{array}\right\}
$$

has a solution.

b) If the LP-system has a D-feasible solution (u,v) such that $v(t) \geq \pi \vec{v}, t \geq 1$ for some $\bar{v} \in$ int $\left(R_{+}^{n}\right)$, then the system

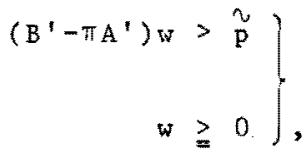

has a solution.

c) If for some $k \geq 1$ and $\bar{v} \in$ int $\left(R_{+}^{n}\right)$, the system

$$
\left.\begin{array}{rl}
B^{\prime} u(t)-A^{\prime} u(t+1)-v(t) & =\left(\frac{1}{\rho}\right)^{t} p(t) \\
u(t) & \geq 0 \\
v(t) & \geq\left(\frac{1}{\rho}\right) t-v
\end{array}\right\} t \geq K,
$$

has a solution, then the system

$$
\left.\begin{array}{rl}
\left(B^{\prime}-\frac{1}{\rho} A^{\prime}\right) w & \geq \frac{2}{p} \\
w & \geq 0
\end{array}\right\},
$$

has a solution. 


\section{Proof.}

The theorem follows from theorem 3.8 and from the symmetry between the primal and the dual system.

\subsection{Remark.}

For a LP-system (P- or D-directed; $f(t) \rightarrow \mathfrak{f} ; t \rightarrow \infty ; p(t) \rightarrow \mathrm{p}$,

$t+\infty)$ the theorems 3.8 and 3.9 may be resumed as follows:

a) If the LP-system is $\mathrm{p}^{\circ}$-feasible then the system

$$
\left.\begin{array}{rl}
\left(B-\frac{1}{\rho} A\right) z & \leqq \\
z & \geq 0
\end{array}\right\},
$$

has a solution.

b) If the LP-system is D-feasible then the system

$$
\left.\begin{array}{rl}
\left(B^{\prime}-\pi A^{\prime}\right) w & > \\
p \\
w \geqq 0
\end{array}\right\},
$$

has a solution.

c) If the LP-system is $\mathrm{p}^{\circ}$-regular, then the system

$$
\left.\begin{array}{rl}
\left(B-\frac{1}{\rho} A\right) z & <\stackrel{\tilde{f}}{ } \\
z & \geqq 0
\end{array}\right\},
$$

has a solution.

d) If the Lip-system is D-regular then the system

$$
\left.\begin{array}{r}
\left(B^{\prime}-\pi A^{\prime}\right) \omega>\widetilde{p} \\
\omega \geq 0
\end{array}\right\},
$$


has a solution.

e) If the LP-system is virtually superregular, then the systems

$$
\left.\begin{array}{rl}
(B-\pi A) z & <\tilde{f} \\
z & \geq 0
\end{array}\right\},
$$

have a solution.

From the next two theorems one may derive a relation between $(3.10 .3)$ and $(3.10 .5)$ and between $(3.10 .4)$ and $(3.10 .6)$.

\subsection{Theorem.}

If the system

$$
\left.\begin{array}{rl}
(B-\gamma A) z & \leqq ‡-h \\
z & \geqq 0
\end{array}\right\},
$$

belonging to a LP-system (P- or D-directed; $f(t) \rightarrow \tilde{f}, t \rightarrow \infty$ ), has a solution for some $\gamma>0$ and $h \in R_{+}^{m}$, then the system

$$
\left.\begin{array}{rl}
(B-\alpha A) z & \leqq \\
- & -\frac{\dot{\gamma}_{h}}{\alpha} \\
z & \geqq 0
\end{array}\right\},
$$

has a solution for every $\alpha \geqq \gamma$.

Proof.

First consider the case that the LP-system is p-directed. If $z$ satisfies $(3.11 .1)$ for some $\gamma>0, h \in R_{+}^{\mathrm{m}}$, then $z$ also 
satisfies

$$
-\gamma A z \leqq \tilde{f}
$$

This implies that for every $\alpha \geqslant \gamma$ the following inequality holds:

$$
-(\alpha-\gamma) A z \leqq\left(\frac{\alpha}{\gamma}-1\right) \tilde{f}
$$

Addition of $(3.11 .1)$ and $(3.11 .3)$ gives

$$
(B-\alpha A) z \leq\left(\frac{\alpha}{\gamma}\right) \tilde{f}-h
$$

Hence it appears that $(3.11 .2)$ has a solution.

For the case that the LP-system is not p-directed we define the diagonal $n \times n$-matrix $\Lambda$ in the following manner

$$
\left.\begin{array}{lll}
\lambda_{j j}:=1, & \text { if } & a_{j} \geqq 0 \\
\lambda_{j j}:=0, & \text { if } & a, j \geqq 0
\end{array}\right\} .
$$

The D-directedness of the LP-system then implies

$$
\left.\begin{array}{l}
B \Lambda \geq 0 \\
A(I-\Lambda) \geq 0
\end{array}\right\} .
$$

If $z \in 1_{+}^{\mathrm{n}}$ satisfies $(3.11 .1)$ for some $\gamma>0, h \in R_{+}^{n}$, then $(3.11 .1)$ and $(3.11 .4)$ imply for every $\alpha \geqq \gamma$ :

$$
B\left(\frac{Y}{\alpha} \Lambda_{z}+(I-\Lambda) z\right)-A(Y z+\alpha(I-\Lambda) z) \leqq \tilde{f}-h
$$

Defining $\tilde{z}:=\gamma / \alpha \Lambda z+(I-\Lambda) z$, it appears that

$$
(B-\alpha A) z \leqq z-h .
$$

Hence we conclude that $(3.11 .2)$ has a solution for every $\alpha \geqq \gamma$. 


\subsection{Theorem.}

If the system

$$
\left.\begin{array}{rl}
\left(B^{\prime}-\gamma A^{\prime}\right) w & \geq \stackrel{a}{p+q} \\
w & \geq 0
\end{array}\right\},
$$

belonging to a LP-system ( - or D-directed; $p(t) \rightarrow \stackrel{p}{p}, t+\infty)$ has a solution for some $\gamma>0$ and $q \in R_{+}^{n}$, then the system

$$
\left.\begin{array}{rl}
\left(B^{\prime}-\alpha A^{\prime}\right) w & \geq \frac{p}{p}+\frac{\alpha}{\gamma} q \\
w & \geq 0
\end{array}\right\},
$$

has a solution for every $a \in[0, Y]$.

Proof.

This theorem follows from theorem 3.11 and from the symmetry between the primal and dual system.

\subsection{Theorem.}

For a LP-system $(f(t)=\tilde{f}, p(t)=\widetilde{p}, t \geq 1)$ the following properties hold:

a) If the system

$$
\left.\begin{array}{rl}
\left(B-\frac{1}{\rho} A\right) z & \leq z \\
z & \geq 0
\end{array}\right\},
$$

has a solution then the LP-system is $\mathrm{p}^{\circ}$-reasible.

b) If the system

$$
\left.\begin{array}{rl}
\left(B-\frac{1}{\rho} A\right) z & <y \\
z & \geq 0
\end{array}\right\}
$$


has a solution then the LP-system is $\mathrm{p}^{\mathrm{o}}$-regular.

c) If the system

$$
\left.\begin{array}{rl}
\left(B^{\prime}-\pi A^{\prime}\right) w & \geq \stackrel{p}{p} \\
w & \geq 0
\end{array}\right\},
$$

has a solution then the LP-system is D-feasible.

d) If simultaneously the systems

$$
\begin{aligned}
& \left.\begin{array}{rl}
(B-\pi A) z & <\mathscr{t} \\
z & \geq 0
\end{array}\right\}, \\
& \left.\begin{array}{rl}
\left(B^{\prime}-\frac{1}{\rho} A^{\prime}\right) w & >\underset{p}{p} \\
w & \geq 0
\end{array}\right\},
\end{aligned}
$$

have a solution and the LP-system is P- or D-directed and $p \pi<1$, then the LP-system is virtually superregular.

\section{Proof.}

If $z$ satisfies $(3.13 .1)$ then $x(t):=p^{t} z, t \geq 1$ is a P-feasible solution.

The properties b) and $c$ ) may be proved in a similar manner. If $\rho \pi<1$ and if the LP-system is $P$ - or D-directed then by virtue of theorem 3.11 and 3.12 the solvability of (3.13.4) and (3.13.5) imply the solvability of the systems (3.13.2) and (3.13.3) resp. Further proof that the conditions of an virtually superregular are satisfied, can be given in a similar manner as done at property a).

\subsection{Remark.}

The next theorems include some statements about the boundness of the set of feasible solutions. We restrict ourselves to the most 
relevant question, namely: in which circumstances all p-feasible solutions are $p-d o m i n a t e d$ and all D-feasible solutions $\pi$-dominated.

\subsection{Proposition.}

If the LP-system has a P-feasible solution ( $x, y)$ such that

$$
y(t) \geqq \rho^{t} y, t \geq k
$$

for some $k \geq 1$ and $y \in \operatorname{int}\left(\mathrm{R}_{+}^{\mathrm{m}}\right)$, and if the linear system

$$
\left.\begin{array}{rl}
\left(B-\frac{1}{p} A\right) z & \leq 0 \\
z & \geq 0
\end{array}\right\}
$$

has a solution, then a p-feasible solution exists which is not o-dominated.

\section{Proof.}

The positivity of $y$, the homogeneity and the solvability of the system ( 3.15 .2$)$ imply that a $z$ exists satisfying

$$
\left.\begin{array}{rl}
B z & \leqq y \\
\left(B-\frac{1}{\rho} A\right) z & \leqq 0 \\
z & \geq 0
\end{array}\right\} .
$$

Now let $(x, y)$ be a P-feasible solution satisfying (3.15.1), so that

$$
\begin{aligned}
& B x(t)-A x(t-1) \leqq \rho^{t} f(t), \quad t=1, \ldots, K, \\
& B x(t)-A x(t-1)+\rho^{t} y \leqq \rho^{t} f(t), \quad t>K,
\end{aligned}
$$

then (3.15.3) and (3.15.5) imply: 


$$
B\left(x(t)+(t-K) \rho^{t} z\right)-A\left(x(t-1)+(t-1-K) \rho^{t-1} z\right) \leqq \rho^{t} f(t), \quad t>K .
$$

Hence it appears that $\{\tilde{x}(t)\}_{1}^{\infty}$ defined as

$$
\left.\begin{array}{ll}
\Psi(t):=x(t) & t=1,2, \ldots, k \\
\tilde{x}(t):=x(t)+(t-K) \rho^{t} z, & t>K
\end{array}\right\} .
$$

is a P-feasible solution, which is not p-dominated because of the fact that $z \geq 0$.

\section{16 Proposition.}

If the LP-system has a P-feasible solution such that

$$
B x(K)-A x(K-1)<p k(K) \text {, for some } K \geq 1 \text {, }
$$

and if the linear system

$$
\left.\begin{array}{rl}
\left(B-\frac{1}{B} A\right) z & \leqq 0 \\
z & \geq 0
\end{array}\right\}
$$

has a solution for some $\beta>\rho$, then there exists a P-feasible solution which is not p-dominated.

\section{Proof.}

If $x$ is a p-feasible solution which satisfies (3.16.1) for some $k \geq 1$ and if $z$ satisfies $(3,16.2)$ for some $\beta>0$, it may be shown that $\{\tilde{x}(t)\}_{1}^{\infty}$ defined by

$$
\left.\begin{array}{ll}
\tilde{x}(t):=x(t), & t=1,2, \ldots k-1 \\
\tilde{z}(t):=x(t)+\beta^{t} z, & t_{\geqq} K
\end{array}\right\},
$$


is a p-feasible solution which is not p-dominated, because of the fact that $\beta>\rho$ and $z \geq 0$.

\subsection{Proposition.}

All P-feasible solutions of a LP-system (P- of D-directed; Pfeasible) are $\rho$-dominated, if the system

$$
\left.\begin{array}{rl}
\left(B-\frac{1}{\rho} A\right) z & \leqq 0 \\
z & \geqq 0
\end{array}\right\},
$$

has no solution.

\section{Proof.}

The supposition that $(3.17 .1)$ is unsolvable, is equivalen.t with the supposition that the system

$$
\left(\begin{array}{c}
B-\frac{1}{\rho} A \\
-I
\end{array}\right) z \leq 0,
$$

has no solution.

By virtue of Stiemke's theorem ${ }^{(*)}$, this implies that the system

$$
\left.\begin{array}{rl}
\left(B^{\prime}-\frac{1}{\rho} A^{\prime}\right) \tilde{u}-\tilde{v} & =0 \\
\tilde{u}, \tilde{v}>0
\end{array}\right\},
$$

has a solution.

Hence there also exists an $\alpha \in] 0, \rho[$ (viz. close enough to $\rho$ ) such that

(*) Stiemke's theorem: the system of 1 inear equalities $A x=0$, $x>0$, has a solution if and only if the system $A^{\prime} u>0$ has no solution. 


$$
\left.\begin{array}{r}
\left(B^{\prime}-\frac{1}{\alpha^{\prime}}\right) u-v=0 \\
u, v>0
\end{array}\right\},
$$

has a solution.

Now let $(x, y)$ be a p-feasible solution, then by virtue of proposition 3.6 it follows that

$$
\left.\begin{array}{rl}
\left(B-\frac{1}{\alpha} A\right) \bar{x} & \leqq \frac{1}{\alpha} A x(0)+\sum_{t=1}^{T+1}\left(\frac{\rho}{\alpha}\right)^{t} f(t) \\
\bar{x} & \geqq \sum_{t=1}^{T}\left(\frac{1}{\alpha}\right)^{t} x(t)
\end{array}\right\},
$$

has a solution for every $T \geqq 1$. Choosing $\alpha \in 10, \rho[$ in such a way that (3.17.2) has a solution, and multiplying (3.17.3) by $\alpha^{\mathrm{T}}$, one may conclude that for every $P$-feasible solution $x$ and for every $\mathrm{T} \geqq 1$ the system

$$
\left.\begin{array}{rl}
\left(B-\frac{1}{\alpha} A\right) \bar{x} & \leqq \rho^{T}\left(\left(\frac{\alpha}{\rho}\right) \frac{T}{\alpha} A x(0)+\left(\frac{\rho}{\alpha}\right) \sum_{t=0}^{T}\left(\frac{\alpha}{\rho}\right)^{t} f(T+1-t)\right) \\
\bar{x} & \geqq x(T)
\end{array}\right\},
$$

is solvable.

Since $\{f(t)\}_{1}^{\infty} \in 1_{\infty}^{m}$ and $\left|\frac{\alpha}{\rho}\right|<1$, the sequence $\sum_{t=0}^{T}\left(\frac{\alpha}{\rho}\right)^{t} f(T+1-t)$ is Consequently a vector $g \in R^{m}$ exists such that for every P-feasible solution $x$ and every $T \geq 1$ the system

$$
\left.\begin{array}{rl}
\left(B-\frac{1}{\alpha} A\right) \bar{x} & \leqq \rho^{T} g \\
\bar{x} & \geqq x(T)
\end{array}\right\},
$$

has a solution. 
If $(\bar{u}, \bar{v})$ is a solution of $(3.17 .2)$, then by multiplying (3.17.5) by $(\bar{u}, \bar{v})^{\prime}$, one can derive that

$$
\left.\begin{array}{l}
\bar{v} \cdot \bar{x} \leqq \rho^{T} \bar{u}^{\prime} g \\
\bar{v} \cdot \bar{x} \geqq \bar{v}^{\prime} x(T)
\end{array}\right\},
$$

has a solution for every $P$-feasible solution $x$ and every $T \geq 1$. since $\bar{y}>0$, this implies that every p-feasible solution is $\rho$-dominated.

\subsection{Remark.}

From the last two propositions it appears that for a lp-system ( $P$ - or D-directed) the unsolvability (3.17.J) implies that (3.16.2) has no solution for every $\beta>\rho$. This result can also be obtained from theorem 3.11. Namely, if $(3.11 .1)$ has a solution for some $\gamma: \frac{1}{\beta}<\frac{1}{\rho}$ and $h=0$, then $(3.11 .2)$ has a solution for $\alpha=\frac{1}{\rho}$. The next two theorems give a summary of the latter three propositions for the primal and dual system.

\subsection{Theorem.}

Every P-feasible solution of a IP-system (P- or D-directed; Pregular) is p-dominated if and only if the system of linear inequalities

$$
\left.\begin{array}{rl}
\left(B-\frac{1}{0} A\right) z & \leqq 0 \\
z & \geq 0
\end{array}\right\},
$$

has no solution.

\subsection{Theorem.}

Every D-feasible solution of a LP-system (P-or D-directed; Dregular) is p-dominated if and only if the system of linear inequalities 


$$
\left.\begin{array}{rl}
\left(B^{\prime}-\pi A^{\prime}\right) w & \geq 0 \\
w \geq 0
\end{array}\right\},
$$

has no solution.

\subsection{Theorem.}

For a LP-system ( $P$ - or D-directed; P- and D-regular; $\rho \pi<1$ ) the following properties hold:

a) if every P-feasible solution is p-dominated, then not all $D-f e a s i b l e$ solutions are $\pi$-dominated.

b) if every D-feasible solution is $\pi$-dominated, then not all $P-f e a s i b l e$ solutions are $\rho$-dominated.

\section{Proof.}

a) If all of the p-feasible solutions are p-dominated, then theorem $3.19 \mathrm{implies}$ that

$$
\left.\begin{array}{rl}
\left(B-\frac{1}{\rho} A\right) z & \leq 0 \\
z & \geq 0
\end{array}\right\}
$$

is unsolvable.

By virtue of stiemke's theorem this implies that

$$
\left.\begin{array}{rl}
\left(B^{\prime}-\frac{1}{\rho} A^{\prime}\right) w & >0 \\
w & >0
\end{array}\right\}
$$

has a solution.

Since $\frac{1}{\rho}>\pi$, theorem 3.12 implies that the system

$$
\left.\begin{array}{rl}
\left(B^{\prime}-\pi A^{\prime}\right) w & \geq 0 \\
w & \geq 0
\end{array}\right\},
$$


is solvable.

By virtue of theorem 3,20 , one can conclude that not all Dfeasible solutions are $\pi$-dominated.

b) This property follows from the symetry between the primal and from the dual system and property a). 


\section{PARTIAL OBJECTIVE FUNCTIONS.}

\subsection{Introduction.}

In this chapter, the feasibility of the primal sysceem is related to certain properties of the dual partial objective functions and, the other way round, the feasibility of the dual system is related to certain properties of the primal. partial objective functions. Previously, we introduce the concept of consistence:

- the primal system is called consistent, or the LP-system is called p-consistent, if there is a p-feasible solution $x$, for which the partial objective functions $\left\langle P_{\pi}, x_{T}, T \geqslant 1\right.$ have a lower bound. Consequently, a P-feasible solution which possesses this property is called a p-consistent solution.

- the dual system is called consistent, or the LP-system is called D-consistent, if there is a D-feasible solution $u$, for which the partial objective functions $\left\langle f_{p}^{0}, u\right\rangle_{T}, T \geq 1$ have an upper bound. A D-feasible solution with this property is called a D-consistent solution.

These denominations are chosen because only in a LP-system which is P-consistent, it is sensible to search for a p-optimal solution. A similar statement can be given with respect to D-optimal solutions.

In the following expressions like $\sum_{t=K}^{L} a(t)^{\prime} b(t)$, where $a, b \in 1^{k}$ and $L \geq K \geq 1$, will be denoted by $\langle a, b\rangle_{K, L}$.

A feasible solution of a LP-system will be denoted by $((x, y),(u, v))$, where $(x, y)$ is the P-feasible solution and $(u, v)$ the D-feasible solution.

\subsection{Proposition.}

For every feasible solution $((x, y),(u, v))$ of a LP-system the following equalities hold: 


$$
\begin{aligned}
\left\langle\mathrm{P}_{\pi}, \mathrm{x}\right\rangle_{K, L}=\left\langle\mathrm{f}_{\rho}, u\right\rangle_{K, L} & +u(K)^{\prime} \mathrm{Ax}(\mathrm{K}-1)-\langle\mathrm{u}, \mathrm{y}\rangle_{K, L}- \\
& -\langle v, x\rangle_{K, L}-u(L+1)^{\prime} A x(L), L L_{K} \geq 1
\end{aligned}
$$

\section{Proof.}

If $((x, y),(u, v))$ is a feasible solution, then for every pair of periods $\mathrm{L} \geqq \mathrm{K} \geq 1$ :

$$
\begin{aligned}
& \left\langle P_{\pi}, x\right\rangle_{X, L}=\sum_{t=K}^{L}\left(B^{\prime} u(t)-A^{\prime} u(t+1)-v(t)\right)^{\prime} x(t)= \\
& \left.=\sum_{t=K}^{L}(u(t))^{\prime}(B x(t)-A x(t-1))-v(t)^{\prime} x(t)\right)+ \\
& +u(K)^{\prime} A x(K-1)-u(L+1)^{\prime} A x(L)=
\end{aligned}
$$

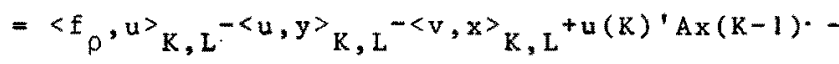

$$
\begin{aligned}
& -u(L+1)^{\prime} A x(L) .
\end{aligned}
$$

After regrouping the equalities $(4.2 .1)$ follow.

\subsection{Proposition.}

For every feasible solution $((x, y),(u, v))$, of a LP-system (P-directed) the following inequalities hold.

$$
\begin{aligned}
& \left.\left\langle P_{\pi}, x\right\rangle_{K, L} \leqq\left\langle E_{\pi}, U\right\rangle{ }_{K, L}{ }^{+u(K) ' A x(K-1)-\langle u, y\rangle} K, L{ }^{-\langle v, x\rangle} K, L\right\} \\
& I \geq K \geq 1 . \quad(4 \cdot 3 \cdot 1)
\end{aligned}
$$

\section{Proof.}

since the LP-system is P-directed, every P-feasible solution $(x, y)$ satisfies

$$
-A x(t) \leqq \rho^{t+1} f(t+1), \quad t \geqq 0
$$

Since $u \in 1_{+}^{m}$ the inequalities $(4.3 .1)$ follow from (4.2.1) and $(4.3 .2)$. 


\subsection{Proposition.}

For every feasible solution $((x, y),(u, v))$ of a LP-system (Ddirected) the following inequalities hold:

$$
\left\langle P_{\pi}, x\right\rangle_{K, L} \leqq\left\langle f_{\rho}, u\right\rangle_{K, L}+u(K) A^{\prime}(K-1)-\langle u, y\rangle_{K, L}-\langle v, x\rangle_{K, L-1}, L \geq K \geqq 1 .
$$

$$
(4.4 .1)
$$

\section{Proof.}

The D-directedness implies that every D-feasible solution $(u, v)$ satisfies

$$
A^{\prime} u(t+1)-v(t) \geq 0, \quad t \geq 1
$$

Since $(x, y) \in 1_{+}^{n+1}+1_{+}^{m}$ one can derive the inequalities $(4.4,1)$ from $(4.2 .1)$ and $(4.4 .2)$.

\section{5 Theorem.}

For every feasible solution $((x),(u))$ of the LP-system the following properties hold:

a) If the LP-system is P-directed then

$$
\left.\left\langle\mathrm{P}_{\pi}, \mathrm{X}\right\rangle_{\mathrm{T}} \leqq\left\langle\mathrm{f}_{\rho}^{0}, \mathrm{U}\right\rangle_{\mathrm{T}+1}, \mathrm{~T}\right\rangle_{\mathrm{I}}
$$

b) If the LP-system is D-directed then

$$
\left.\left\langle\mathrm{P}_{\pi}, \mathrm{x}\right\rangle_{\mathrm{T}} \leqq\left\langle f_{\rho}^{0}, \mathrm{U}\right\rangle_{\mathrm{T}}, \mathrm{T}\right\rangle
$$

c) If the LP-system is P-or D-directed and $\left\langle P_{T}, x\right\rangle_{\infty}$ and $\left\langle f_{p}^{0}, u\right\rangle_{\infty}$ exist, then

$$
\left\langle P_{\pi}, x\right\rangle_{\infty} \leqq\left\langle E_{\rho}^{0}, u\right\rangle_{\infty}
$$

Proof.

This theorem follows from $4.3,4.4$ and from the non-negativity of feasible solutions. 


\section{6 corollary.}

The primal partial objective functions of a LP-system(P-or Ddirected; P-feasible; D-consistent) are bounded above.

The dual partial objective functions of a LP-system (P-or Ddirected; $P^{\circ}$-consistent; $D$-feasible) are bounded below.

\subsection{Theorem.}

If a LP-system (P-or D-directed) possesses a regular solution which is constistent, then $\rho \pi<1$.

\section{Proof.}

The regularity of a LP-system implies that a regular solution $((x, y),(u, v))$ exists, such that there are vectors $x$, vint $\left(R_{+}^{n}\right)$, u, yeint $\left(R_{+}^{m}\right)$, for which

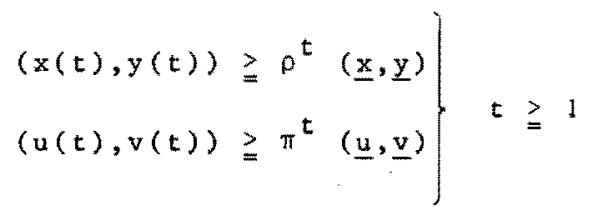

Now suppose that this regular solution $((x, y),(u, v))$ is consistent as well; i.e. a lower bound $M_{p}$ exists for the primal partial objective functions and an upper bound $M_{D}$ for the dual partial objective functions. For these numbers $M_{P}$ end $M_{D}$ we have:

$$
\left.\begin{array}{l}
\left\langle P_{\pi}, x\right\rangle_{T} \geqq M_{P} \\
\left\langle f_{\rho}^{O}, u\right\rangle_{T} \leqq M_{D}
\end{array}\right\} t \geqq 1
$$

Assuming that the LP-system is P-or D-directed, substitution of $(4.7 .1)$ and $(4.7 .2)$ in $(4.3 .1$ or $(4.4 .1)$ gives the inequa1ities:

$$
\sum_{t=1}^{T}(0 \pi)^{t}(\underline{u}, \underline{v})^{\prime}(\underline{y}, \underline{x}) \leqq M_{D}-M_{p}, \quad t \geq 1
$$


Since $\rho, \pi,(\underline{x}, \underline{y})$ and $(\underline{u}, \underline{v})$ are all positive, the inequalities $(4.7 .3)$ imply, that $p \pi<1$.

\subsection{Remark.}

Henceforth we shall mainly consider the situation that $0 \pi<1$. In that case, for every $\rho$-dominated $x \in 1^{n}$ and every $\pi$-dominated $u \in 1^{m}: x_{R} \in 1_{1}^{n}$ and $u_{\rho} \in 1_{1}^{m}$.

Since $f^{\alpha} \in 1_{\infty}^{m}$ and $p \in 1_{\infty}^{n}$, this implies that, for every $\rho$-dominated p-feasible $x$ and for every $\pi$-dominated $D$-feasible $u$, the sequences $\left\{\left\langle\mathrm{p}_{\pi}, \mathrm{x}\right\rangle\right\}_{\mathrm{T}=1}^{\infty}$ and $\left\{\left\langle\mathrm{f}_{\rho}^{\circ}, u\right\rangle_{T}\right\}_{\mathrm{T}=1}^{\infty}$ resp. are convergent.

\subsection{Theorem.}

A P-feasible solution $x$ of a LP-system(P-or D-directed; $P$-feasible; D-regular; $p \pi<1$ ) is consistent if and only if $x_{\pi} \in 1_{1}^{n}$.

\section{Proof.}

Necessary: Let $M_{p}$ be a lower bound for the primal partial objective functions of a p-consistent solution $x$ and let $(u, v)$ be a D-regular solution.

Since the LP-problem is P-or D-directed the theorems 4.3 or 4.4 imply:

$$
\begin{aligned}
\sum_{t=1}^{T} v(t)^{\prime} x(t) & \leqq\left\langle f_{\rho}^{o}, u\right\rangle_{T+1}-M_{p} \leqq \\
& \leqq\left\|f^{o}\right\|_{\infty}\left\|_{\rho}\right\|-M_{p}, \quad T \geqq 1 .
\end{aligned}
$$

where the 1 ast inequality follows from the fact that $u_{1 / \pi} \in 1_{\infty}^{m}$ and $p \pi<1$.

Since $v_{1 / \pi} \in \operatorname{int}\left(I_{\infty+}^{n}\right)$ and $x \in 1_{+}^{n}$, the inequalities $(4.9 .1)$ imply:

$$
x_{\pi} \in 1_{1}^{n}
$$

sufficient: Let $x$ be a P-feasible solution such that $x_{\pi} \in 1_{1+}^{n}$. 
Since $p \in 1_{\infty}^{n}$, this supposition implies:

$$
\left\langle\mathrm{P}_{\pi}, \mathrm{x}\right\rangle_{\mathrm{T}} \geqq-\|\mathrm{P}\|_{\infty}\left\|\mathrm{x}_{\pi}\right\|_{1}, \mathrm{~T} \geqq 1 .
$$

Clearly, $x$ is consistent.

\subsection{Theorem.}

A D-feasible solution u of a LP-system (P-or D-directed; $\mathrm{P}^{\circ}$-regular; D-feasible; $p \pi(1)$ is consistent if and only if $u_{p} \in 1_{\infty}^{m}$

\section{Proof.}

This theorem may be proven in a simular way as theorem 4.9 .

\section{11 Remark.}

Up to now it always has been supposed that the primal and dual systems are both feasible. Now, however, we shall start from suppositions implying that one of the systems is not feasible.

\subsection{Proposition.}

If, for a LP-system (D-consistent) a period $\mathrm{T} \geqslant 1$ exists, such that the system of linear inequalities

$$
\left.\begin{array}{rlrl}
B \times(1) & \leqq p f(1)+A x(0) & \\
B x(t+1)-A x(t) & \leqq \rho^{t+1} f(t+1), & t=1,2, \ldots, T \\
x(t) & \geqq 0, & t=1,2, \ldots, T+1
\end{array}\right\},
$$

is nonsolvable, then a lower bound for the dual partial objective functions does not exist.

\section{Proof.}

If $(4.12 .1)$ is nonsolvable, then, by virtue of 
Farkas' theorem $(x)$, it follows that a $\left\{u^{\circ}(t)\right\}_{1}^{T+1}, u^{\circ}(t) \geqq 0$, $t=1,2, \ldots, T+1$ exists, satisfying

$$
\left.\begin{array}{rl}
B^{\prime} u^{0}(t)-A^{\prime} u(t+1) & \geqq 0, t=1,2, \ldots, T \\
B^{\prime} u(T+1) & \geqq 0 \\
\left\langle f_{\rho}^{0}, u\right\rangle_{T+1} & <0
\end{array}\right\} .
$$

Now let $\vec{u}$ be a p-consistent solution and let us define the vectors $u(\lambda):=\{u(t ; \lambda)\}_{t=1}^{\infty}$ for every $\lambda \geqq 0$ by

$$
\left.\begin{array}{ll}
u(t ; \lambda):=\bar{u}(t)+\lambda u^{o}(t), & t=1,2, \ldots, T+1 \\
u(t ; \lambda):=\bar{u}(t), & t>T+1
\end{array}\right\} .
$$

Then $(4,12,2)$ and $(4,12,3)$ imply that the vectors $u(\lambda), \lambda \geqq 0$ are all feasible and, moreover, that

$$
\left\langle f_{\rho}^{0}, u(\lambda)\right\rangle_{S}=\left\langle f_{\rho}^{0}, \bar{u}\right\rangle_{S}+\lambda\left\langle f_{\rho}^{o}, u^{o}\right\rangle_{T+1}, \lambda \geqq 0, \quad S>T .
$$

Since it is supposed that $\bar{u}$ is p-consistent and since $\left\langle f_{\rho}^{0}, u^{0}\right\rangle_{T+1}<0$, we can conclude that a lower bound for the dual partial objective functions does not exist.

\subsection{Proposition.}

If for a LP-system (P-consistent) a period $T \geqq 1$ exists, such that the system of linear inequalities

$$
\left.\begin{array}{rl}
B^{\prime} u(t)-A^{\prime} u(t+1) & \geqq \pi^{t} p(t), t=1,2, \ldots, T \\
u(t) & \geqq 0, \quad t=1,2, \ldots, T+1
\end{array}\right\}
$$

is nonsolvable, then an upper bound for the primal partial objective functions does not exist.

(*) Farkas' theorem (inequality form): The system of linear inequalities $A x \leq b$ has a solution $x \geq 0$ if and only if every $u \gg 0$ satisfying $A^{\prime} u \geq 0$ also satisfies $b^{\prime} u \geq 0$. 


\section{INFERIORITY.}

\subsection{Introduction.}

In this chapter we shall derive some properties with respect to the concept of inferiority. We repeat the definition already given at $\$ 2.5$.

A P-feasible solution $x$ is called inferior with respect to a $p$-feasible solution $\bar{x}$, if an $\varepsilon>0$ and a period $T \geq 1$ exist such that

$$
\left\langle\mathrm{P}_{\pi}, \mathrm{x}\right\rangle_{S} \leqq\left\langle\mathrm{P}_{\pi}, \overline{\mathrm{x}}\right\rangle_{S}-\varepsilon, \quad \mathrm{S} \geq \mathrm{T}
$$

A D-feasible solution $u$ is called inferiar with respect to a $D$-feasible solution $\bar{u}$, if an $\varepsilon>0$ and a period $T \geq 1$ exist such that

$$
\left\langle f_{p}^{0}, u\right\rangle_{S} \geqslant\left\langle f_{\rho}^{0}, \bar{u}\right\rangle_{S}+\varepsilon, \quad S \geqslant T .
$$

\subsection{Theorem.}

For every P-consistent solution $\bar{x}$ of a LP-system(P-or D-directed; p-consistent; $D$-regular; $\rho \pi<1$ ), a number $M \geq\left\|\bar{x}_{\pi}\right\|$, exists such that every P-feasible $x$, with the property that

$$
\left\|x_{\pi}\right\|_{l}^{1, T}>M, \text { for some } T \geq 1
$$

is inferior with respect to $\bar{x}$.

\section{Proof.}

Let $(\bar{u}, \bar{v})$ be a D-regular solution, then proposition 4.3 or 4.4 implies:

$$
\left\langle\mathrm{P}_{\pi}, \mathrm{x}\right\rangle_{\mathrm{S}+1} \leqq\left\|\mathrm{f}_{\rho \pi}^{\mathrm{o}}\right\|_{1}\left\|\overline{\mathrm{u}}_{1 / \pi}\right\|_{\infty}=\langle\overline{\mathrm{v}}, \mathrm{x}\rangle_{\mathrm{S}}, \quad \mathrm{S} \geq 1
$$

for every P-feasible solution $x$, Since $\bar{v}_{1 / \pi} \in \operatorname{int}\left(1_{\infty++}^{n}\right)$, it 
follows from $(5.2 .2)$ that a number $N_{1}>0$ exists such that

$$
\left\langle\mathrm{P}_{\pi}, \mathrm{x}\right\rangle_{\mathrm{S}+1} \leqq\left\|\mathrm{f}_{\rho \pi}^{\mathrm{o}},\right\| \overline{\mathrm{u}}_{1 / \pi}\left\|_{\infty}-\mathrm{N}_{1}\right\| \mathrm{x}_{\pi} \|_{1}, \mathrm{~S}, \mathrm{~S} \geq 1
$$

for every p-feasible $x$.

Let $\bar{x}$ be a p-consitent solution, then ( $\$ 4.1)$ a number $\mathrm{N}_{2} \geq 0$ exists such that

$$
\left\langle\mathrm{P}_{\pi}, \bar{x}\right\rangle_{S+1} \geq-\mathrm{N}_{2}, \quad \mathrm{~S} \geq 1
$$

From (5.2.3) and $(5.2 .4)$ we may conclude that for every P-feasible $x$ :

$$
\left\langle\mathrm{P}_{\pi}, \mathrm{x}\right\rangle_{\mathrm{S}+1}-\left\langle\mathrm{P}_{\pi}, \overline{\mathrm{x}}_{\mathrm{S}+1} \leqq \mathrm{ff}_{\rho \pi}^{\circ}\|,\| \bar{u}_{1 / \pi}\left\|_{\infty}+\mathrm{N}_{2}-\mathrm{N}_{1}\right\| \mathrm{x}_{\pi} \|_{1}, \mathrm{~S}, \mathrm{~s} \geq 1 .\right.
$$

Putting $M:=\left(\left\|f_{\rho \pi}^{o}\right\|,\left\|\bar{u}_{1 / \pi}\right\|_{\infty}+N_{2}\right) / N_{1}$, then $(5.2 .5)$ implies

$$
\left\langle\mathrm{P}_{\pi}, \mathrm{x}_{\mathrm{S}}-\left\langle\mathrm{P}_{\pi}, \overline{\mathrm{x}}\right\rangle_{\mathrm{S}} \leq \mathrm{N}_{1}\left(\mathrm{M}-\mathrm{H} \mathrm{x}_{\pi}, \mathrm{I}_{1}\right), \mathrm{S}>\mathrm{T} \geqq 1,\right.
$$

for every D-feasiblex.

From $(5.2 .6)$ we may conclude:

$-\left\|\mathrm{x}_{\pi}\right\| 1 \leqq M$,

- every p-feasible $x$, satisfying $(5.2 .1)$ for some $T \geqq 1$ is inferior with respect to $\bar{x}$.

\subsection{Theorem.}

For every D-consistent solution $\bar{u}$ of a LP-system(P-or Ddirected; D-consistent; P-regular; $0 \pi<1)$, a number $M \geq\left\|\bar{u}_{\pi}\right\|$ exists such that every $D$-feasible $u$, with the property that

$$
\left\|u_{\rho}\right\|_{1}^{1}, T>M, \text { for some } T \geqq 1,
$$

is inferior with respect to $\bar{u}$. 


\section{Proof.}

This theorem follows from 5.2 and from the symmetry between the primal and dual systems.

\subsection{Definitions.}

We consider the LP-system

$$
\left.\begin{array}{rl}
B \times(1) & \leqq \rho f(1)+A x(0) \\
B x(t+1)-A x(t) & \leqq \rho{ }^{t+1} f(t+1), \quad T \geqq 1 \\
\left\langle\rho \pi, x_{T}:\right. & =\sum_{t=1}^{T} \pi^{t} p(t)^{\prime} x(t), T \geqq 1
\end{array}\right\}\{(5.4,1)
$$

for all vectors $x(0), f$ and $p$ from neigbourhoods $\Omega(h) C R_{+}^{n}$, $\Omega(g) \subset 1_{\infty}^{m}$ and $\Omega(q) \subset 1_{\infty}^{n}$ of vectors $h \in R_{+}^{n}, g \in 1_{\infty}^{m}$ and $q \in 1_{\infty}^{n}$, further to be described.

With regard to the fixed matrices $A$ and $B$, we suppose that they are such, that a $f$ or p exists for which the LP-system (5.4.1) is P-or D-directed. For the fixed quantities o and $\pi$, it is supposed that $\rho \pi<1$.

With regard to the vectors $h \in R_{+}^{n}, g \in 1_{\infty}^{m}$ and $q \in 1_{\infty}^{n}$, we suppose that the following conditions are met:

a) for $x(0)=h, f=g$ and $p=q$, the LP-system $(5.4 .1)$ is $P-o x D-$ directed and $P-$ and D-regular,

b) a number. $B>1$ and vectors $(x, \tilde{y}) \in 1_{+}^{\mathrm{n}} \times 1_{+}^{\mathrm{m}},(\tilde{u}, \tilde{v}) \in 1_{+}^{\mathrm{m}} \times 1_{+}^{\mathrm{n}}$ exist, such that $\left(\tilde{x}_{1 / \beta \rho}, \tilde{y}_{1 / \beta p}\right) \in 1_{\infty++}^{n} \times \operatorname{int}\left(1_{\infty+}^{m}\right),\left(\tilde{u}_{1 / \beta \pi},{ }_{v}^{u}{ }_{1 / \beta \pi}\right) \in 1_{\infty++}^{m} \times \operatorname{int}\left(1_{\infty+}^{n}\right.$ and

$$
\left.\begin{array}{l}
B \tilde{x}(t+1)-A \tilde{x}(t)+\tilde{y}(t+1)=(B p)^{t+1} g(t+1) \\
B^{\prime} \tilde{u}(t)-A \cdot \tilde{u}(t+1)-v(t)=(\beta \pi)^{t} q(t)
\end{array}\right\} t \geqslant k,
$$


for some period $k \geqq 1$.

Clearly, in that case, neighbourhoods $\Omega(h) \subset R_{+}^{n}, \Omega(g) \subset 1_{\infty}^{m}, \Omega(q) \subset 1_{\infty}^{n}$ and positive numbers $\delta_{1}$ to $\delta_{4}$ exist, such that for every $x(0) \in \Omega(h), f \in \Omega(g)$ and $p \in \Omega(q)$ :

c) $\hat{B} \tilde{x}(t+1)-A \tilde{x}(t)+(B \rho)^{t+1} \delta_{1} e \leq(B p)^{t+1} f(t+1), \quad t \geq K$,

$B^{\prime} \tilde{u}(t)-\hat{A} \tilde{u}(t+1)-(\beta \rho)^{t} \delta_{2} e \geqq(B \pi)^{t} p(t), \quad t \geq K$,

$\tilde{x}, \tilde{u}, \beta, k$, being the same quantities as appearing in $b$ ).

d) $\mathrm{B} \overline{\mathrm{x}}(1)$

$\left.B \bar{x}(t+1)-A x(t)+\rho^{t+1} \delta_{3} e \leqq \rho^{t+1} f(t+1), t \geqq 1\right\}$,

$B^{\prime} \bar{u}(t)-A^{\prime} \bar{u}(t+1)-\pi^{t} \delta_{4} e \geq \pi^{t}(p(t), \quad t \geq 1$

for some fixed $\bar{x} \in 1_{+}^{n} ; \bar{u} \in 1_{+}^{m}$, such that $\bar{x}_{1 / \rho} \in 1_{\infty}^{\mathfrak{n}}$ and $\bar{u}_{1 / \pi} \in 1_{\infty}^{\mathrm{m}}$.

In the following properties the equalities $(5,4.3)$ and

(5.4.5) will be used to construct, from every given p-feasible solution, a sequence of substitute P-feasible solutions. Next a comparison will drawn between the values of the corresponding objective functions.

We define:

e) $\Omega\left(g^{0}\right):=\left\{\left(f(1)+\frac{1}{p} A x(0), f(2), \ldots, f(t) \mid x(o) \in \Omega(h), f \in \Omega(g)\right\}\right.$, where: $g^{0}:=\left(g(1)+\frac{1}{\rho} A h, g(2), \ldots, g(t), \ldots\right)$ '

f) For every $f^{\circ} \in \Omega\left(g^{\circ}\right), z \in 1_{+}^{m}$ the set: $X\left(f^{\circ}, z\right):=\left\{\begin{array}{ll}x_{x \in 1}+\mid \begin{array}{ll}b x(1) & \leq \rho f^{\circ}(1)+z(1) \\ B \times(t+1)-A x(t) \leq \rho^{t+1} f^{\circ}(t+1)+z(t+1), t \geq 1\end{array}\end{array}\right\}$

g) For every $T \geqq K$ the sequences of numbers $\theta(T):=\{\theta(t ; T)\}_{t=K-1}^{\infty}$ and $\tilde{\theta}(T):=\{\tilde{\theta}(t ; T)\}_{t=K-1}^{\infty}:$ 


$$
\begin{aligned}
& \left.\begin{array}{l}
\theta(0 ; T):=1-B^{I-T} \\
\theta(t ; T):=1-B^{t-T}, t=1,2, \ldots, T \\
\theta(t ; T):=0 \quad, t>T
\end{array}\right\} \text { in case that } K=1, \\
& \left.\begin{array}{l}
\theta(K-1 ; T):=1 \\
\theta(t ; T) \quad:=1-\beta^{t-T}, t=K, K+1, \ldots, T \\
\theta(t ; T) \quad:=0 \quad, t>T
\end{array}\right\} \text { in case that } K>1, \\
& \left.\begin{array}{ll}
\tilde{\theta}(t ; T):=\beta^{-(T+1)}, t=K-1, K, \ldots, T \\
\tilde{\theta}(t ; T):=\beta^{-(t+1)}, t>T
\end{array}\right\} \text { in both cases. }
\end{aligned}
$$

h) For every $x \in X\left(\Omega\left(g^{\circ}\right), 1_{+}^{m}\right)$ and every $T \geq k$, the vectors. $\hat{x}(T, x):=\{\hat{x}(t ; T, x)\}_{t=1}^{\infty}$ by

$\hat{x}(t ; T, x):=x(t), t=1,2, \ldots, k-1, \quad$ provided $k>1$

$$
\left.\begin{array}{rl}
\hat{x}(t ; T, x):=\theta(t ; T) \Lambda x(t) & +\theta(t-1 ; T)(I-\Lambda) x(t)+ \\
& +\tilde{\theta}(t ; T) \Lambda x(t)+\tilde{\theta}(t-1 ; T)(I-\Lambda) \tilde{x}(t), t \geq K
\end{array}\right\}
$$

where $A$ is the diagonal $n \times n-m a t r i x$ as defined in proposition 3.5 , and $\tilde{x}$ the vector of property $5.4-c$.

\subsection{Proposition.}

If the vectors $h \in R_{+}^{n}, g \in 1_{\infty}^{m}$ and $q \in 1_{\infty}^{n}$ satisfy the conditions $5.4-a, b$, then neighbourhoods $\Omega(h) \subset R_{+}^{n}, \Omega(g) \subset 1_{\infty}^{m}, \Omega(q) \subset 1_{\infty}^{n}$ and positive numbers $N_{1}$ to $N_{6}$ exist such that:

for every $(f, p) \in \Omega(g) \times \Omega(q)$, for which the LP-system(5.4.1) is $P$-or $D$-directed, and for every $x(0) \in \Omega(h), \quad z \in 1_{+}^{m}$, the vectors $\hat{x}(T ; x), T \geqslant K, x \in X\left(f^{\circ}, z\right)$ as defined by $5.4-h$, possess the following properties:

a) In case $k=1$ :

$$
\left.\begin{array}{ll}
\hat{B} \hat{x}(1 ; T, x)+B\left(B^{K-T} \times(1)-B^{-(T+1)}\right) \\
B \hat{x}(t+1 ; T, x)-A \hat{x}(t ; T, x) & \leq \rho^{t+1} f^{o}(t+1)+z(t+1), t \geq 1
\end{array}\right\}(5.5 .
$$


b) In case $k>1$ :

$$
\begin{aligned}
\hat{B x}(1 ; T, x) & \leqq \rho f^{o}(1)+z(1) \\
\hat{B} \hat{x}(t+1 ; T, x)-A \hat{x}(t ; T, x) \leqq \rho^{t+1} f^{o}(t+1)+z(t+1), t \geqq 1, t \neq K-1 & \\
B \hat{x}(K ; T, x)-A \hat{x}(K-1 ; T, x)+ & \\
& +B\left(B^{K-T} \Lambda x(K)-B^{-(T+1)} \tilde{x}(K) \leqq \rho^{K} f^{o}(K)+z(K)\right.
\end{aligned}
$$

c) In both cases, if $z_{B \pi} \in 1_{1+}^{m}$ :

$$
\begin{aligned}
& \Delta(L, T, x):=\left\langle p_{\eta}, x\right\rangle_{L}-\left\langle p_{\pi}, \hat{x}(T, x)\right\rangle_{L} \leqq \\
& \leq-\beta^{-\mathrm{T}} \mathrm{N}_{1}\left\|\mathrm{x}_{\beta \pi}\right\|{ }_{1}^{\mathrm{K}, \mathrm{T}+1}+(\rho \pi)^{\mathrm{T}} \mathrm{N}_{2}+\beta^{-\mathrm{T}} \mathrm{N}_{3}+\beta^{-\mathrm{T}} \mathrm{N}_{4}\left\|z_{B \pi}\right\|+ \\
& +\beta^{-T_{N}}\|x(K-1)\|_{1}+\beta^{-T_{N}}{ }_{6} \sum_{t=0}^{\infty}(B p \pi)^{t}, L>t \geq K \text {. }
\end{aligned}
$$

\section{Proof.}

As has been explained in $\$ 5.4$, there are neighbourhoods, $\Omega(h) \subset R_{+}^{n}, \Omega(g) \subset 1_{\infty}^{m}, \Omega(q) \subset 1_{\infty}^{n}$ for the vectors $h, g, q$ satisfying $5.4-a, b$, for which the properties $5.4-c, d$ hold. In the proof we shall further depart from these neighbourhoods.

(a) and (b): since the sequences $\{\theta(t ; T)\}_{t=K-1}^{\infty}$ and $\{\forall(t ; T)\}_{t=k-1}^{\infty}$ (5.4-g) are monotonous non-increasing, proposition 3.5 implies: for every $x \in X\left(f^{0}, z\right)$ (def $5,4-f$ ), provided $z \in 1_{+}^{m}$ and $\left(f^{\circ}, p\right) \in \Omega\left(g^{\circ}\right) \times \Omega(q)$ such that the LP-system $(5.4 .1)$ is p-or D-directed, the following inequalities hold:

$$
\begin{array}{cc}
B(\theta(t+1 ; T) \Lambda+\theta(t ; T)(I-\Lambda)) \times(t+1)- & (5.5 .4) \\
-A(\theta(t ; T) \Lambda+\theta(t-1 ; T)(I-\Lambda)) \times(t) \leq \theta(t ; T)\left(\rho^{t+1} f^{0}(t+1)+z(t+1)\right), & t \geqq K .
\end{array}
$$

Moreover, for $\stackrel{2}{x}(5.4-c)$ we have: 


$$
\begin{array}{cc}
B(\tilde{\theta}(t+1 ; T) \Lambda+\tilde{\theta}(t ; T)(I-\Lambda)) \tilde{x}(t+1)- & (5.5 .5) \\
-A(\tilde{\theta}(t ; T) \Lambda+\tilde{\theta}(t-1 ; T)(I-\Lambda)) \times(t) \leqq \tilde{\theta}(t ; T)(B \rho)^{t+1} f^{\circ}(t+1), & t \geq K .
\end{array}
$$

From $(5.5 .4),(5.5 .5), x \in 1_{+}^{m}$ and from the definitions $(5.4-\mathrm{g}, \mathrm{h})$ of $\hat{x}(T, x), \theta(T)$, and $\tilde{\theta}(T)$, it follows:

$$
B \hat{x}(t+1 ; T, x)-A \hat{x}(t ; T, x) \leqq \rho^{t+1} f^{o}(t+1)+z(t+1), \quad t \geqq K .
$$

The validity of the properties a) and b) is further deducted from the above, with the help of the definition of $\hat{x}(T, x), \theta(T)$ and $\tilde{\theta}(T)$.

\section{(c): Defining}

$$
\mathrm{p}^{\lambda}:=\{\Lambda \mathrm{p}(\mathrm{t})\}_{1}^{\infty}
$$

for every $p \in \Omega(q)$, the definition of $\hat{x}(T, x)(5.4-h)$ implies that $\Delta(L, T, X)$ may be expressed as follows:

- In case $\mathrm{K}=1, \mathrm{~T}>\mathrm{K}$ :

$$
\begin{aligned}
& \left.\Delta(\mathrm{L}, \mathrm{T}, \mathrm{x})=\beta^{\mathrm{K}-\mathrm{T}} \pi \mathrm{p}(\mathrm{K})^{\prime} \mathrm{x}(1)+\beta^{-\mathrm{T}}<\left(\beta \mathrm{p}^{\lambda}+\mathrm{p}-\mathrm{p}^{\lambda}\right)\right]_{\pi}, \frac{1}{\beta} \mathrm{x}_{B}{ }^{\prime} \mathrm{K}+1+
\end{aligned}
$$

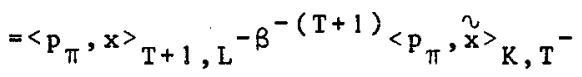

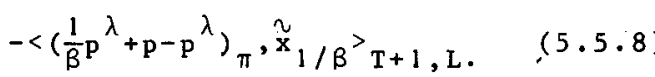

- In case $T=K=1$, the second term of the right-hand side of (5.5.8) will be cancelled.

- In case $\mathrm{T} \gg \mathrm{K}>1$ :

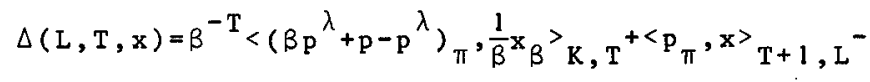

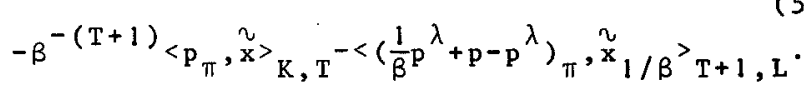

For arbitrary $(x(0), f, p) \in \Omega(h) \times \Omega(g) \times \Omega(q), \quad z \in I_{+}^{m}, x \in x\left(f^{\circ}, z\right)$ and 
$\mathrm{L}>\mathrm{T} \geq 1$, such that:

- the LP-system $(5.4 .1)$ is P-or D-directed,

$-\quad\|x(0)-h\|_{\infty} \leq 1,\|f-g\|_{\infty} \leq 1,\|p-q\|_{1} \leq 1$,

$-\quad z_{\beta \pi} \in 1_{1+}^{\mathrm{m}}$,

the terms of the right-hand side of (5.5.8) and (5.5.9) will be valued down with the help of positive numbers $M_{1}$ to $M_{8}$, which are independent of $x, L$ and $T$. We shall first consider $(5.5 .8)$

The first three terms of the right-hand side of $(5.5 .8)$.

First we put the case that $f^{\circ}$ is such that the LP-system (5.4.1) is not P-directed and so is D-directed. Then, the definitions of $\Lambda$ (3.5) and $\mathrm{p}^{\lambda}(5.5 .7)$ imply:

$$
\left.\begin{array}{c}
\mathrm{p}^{\lambda} \in 1_{\infty-}^{\mathrm{n}} \\
B \Lambda \geq 0 \\
A(I-\Lambda) \geq 0
\end{array}\right\} .
$$

Denoting the sum of the values of the first three right-hand side terms of $(5.5 .8)$ by $\Delta_{1}(L, T, x)$, then $(5.5 .10)$ and the fact that $\beta>1$ and $x \in 1_{+}^{\mathfrak{n}}$ imp 1 : :

$$
\begin{aligned}
\Delta_{1}(L, T, x) \leq\left.\beta \pi_{p}^{K}(K)\right|_{\beta} ^{K-T-1} x(K) & +<p_{\pi}, \beta^{-T-1} x_{\beta}>_{K+1, T^{+}} \\
& +<\left(\frac{1}{\beta} p^{\lambda}+p-p^{\lambda}\right)_{\pi}, x>T+1, L .
\end{aligned}
$$

The second term of the right-hand side will be cancelled when $\mathrm{T}=1$.

We shall value down the right-hand side of $(5.5 .11)$ for the case that $\mathrm{T}>1$.

Since $x \in X\left(f^{\circ}, z\right)$ and $\beta>1$, it follows from (5.5.10) that 


$$
\begin{aligned}
& B B^{t-T} \times(t+1) \quad-B A B^{t-1-T} \times(t) \quad \angle B^{t-T}\left(\rho^{t+1} f(t+1)+z(t+1)\right), t=k-1, \ldots, T-1, \\
& B\left(\frac{1}{\beta} \Lambda+I-\Lambda\right) \times(T+1)-B A \frac{1}{B} \times(T) \quad \leq \rho^{T+1} f(T+1)+z(T+1) \text {; } \\
& B\left(\frac{1}{\beta} \Lambda+I-\Lambda\right) \times(t+1)-B A\left(\frac{1}{\beta} \Lambda+I-\Lambda\right) \times(t) \leqq \rho^{t+1} f(t+1)+z(t+1)
\end{aligned}
$$

$(5.5 .12)$

Denoting $\tilde{u}, \tilde{u}_{1 / \beta \pi} \in 1_{\infty+}^{\text {m }}$ and $\delta_{2}>0$ as the quantities for which property $(5.4-c)$ holds, then:

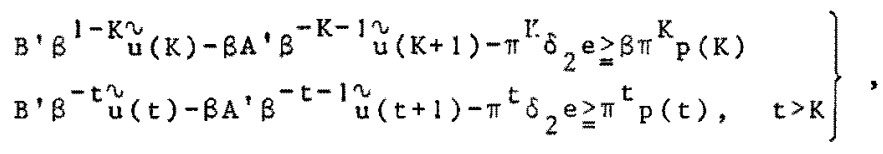

in which the first inequality follows from the supposition that the LP-system $(5,4,1)$ is D-directed.

The whole, consisting of $(5.5 .12)$ and $(5.5 .13)$, may be taken as an LP-system which is D-directed. By applying proposition 4.4 on this LP-system, one can derive the following inequality:

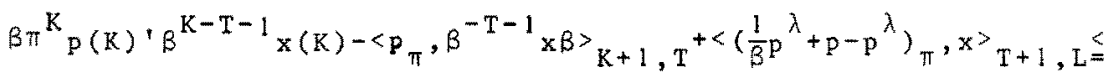

$$
\begin{aligned}
& \leq\left(\beta^{K-1-T} \times(K-1)^{\prime} A^{\prime}+\beta^{K-T}\left(\rho^{K+1} f(K+1)^{\prime}+2(K+1)^{\prime}\right) \beta^{1-K{ }_{u}(K)+}\right. \\
& +B^{-T}<f_{B P}+z_{B}, \vec{u}_{1 / B^{\prime}} K+1, T^{+<f_{P}+z, w_{1 / B} T+1, L}- \\
& -\beta^{-T-2} \delta_{2}\left\|x_{B \pi}\right\|_{1}^{-K, T+1} \text {. }
\end{aligned}
$$

Since $\|f\|<\|g\|_{\infty}+1, u_{1 / \beta \pi} \in 1_{\infty}^{m}, \beta>1, \rho \pi<1$ and $z_{\beta \pi} \in 1_{1+}^{m}$, one can derive from the latter inequality, that positive numbers $M_{1}$ to $M_{5}$ exist such that:

$\Delta_{1}(L, T, X) \leqq B-T\left(M_{1}+M_{2}\left\|z_{B \pi}\right\|{ }_{1}+M_{3} \sum_{t=1}^{T}(B \rho \pi){ }^{t}-M_{4}\|x(K-1)\|_{1}-M_{5}\left\|x_{B \pi}\right\|{ }_{1}^{K, T+1}\right)$.

For the case that $T=K$, the same inequality will be found. 
Now consider the case that $f^{\circ}$ is such that LP-system (5.4.1) is P-directed. Then, $\Lambda$ is defined as the identity matrix; hence:

$$
\Delta_{1}(L, T, x)=\beta^{-T}\left\langle p_{\pi}, x_{\beta}\right\rangle_{K, T}+\left\langle p_{\pi}, x\right\rangle_{T+1}
$$

Exploring the properties of p-directed LP-systems already deducted, the right-hand side of latter equality may be valued down in a similar manner as done above.

\section{The fourth and fifth term of the right-hand side of (5.5.8)}

Since, by assumption,

$$
\|\mathrm{p}\|_{\infty} \leq\|\mathrm{q}\|_{\infty}^{+1}, \tilde{x}_{1 / B p} \in 1_{\infty}^{n^{+}}, \quad \rho \pi<1,
$$

we may conclude that positive numbers $M_{6}$ and $M_{7}$ exist such that

$$
\begin{aligned}
& -\beta^{-T-1}<p_{\pi}, x>{ }_{K}, T^{-\left\langle\left(\frac{1}{\beta} p^{\lambda}+p-p^{\lambda}\right)_{\pi}, x_{1 / B} T+1, L\right.}= \\
& \Leftrightarrow \beta^{-T} M_{6} \sum_{t=0}^{T}(B \rho \pi)^{t}+(\rho \pi)^{T} M_{7} \text {. }
\end{aligned}
$$

\section{The total right-hand side of $(5.5 .8)$}

By substitution of $(5.5 .14)$ and $(5.5 .15)$ in $(5.5 .8)$ we may deduct that property c) holds in case $k=1$.

The right-hand side of $(5.5 .9)$

We may treat the right-hand side of (5.5.9) in a similar manner as the right-hand side of (5.5.8); i.e. (5.5.11) will change into

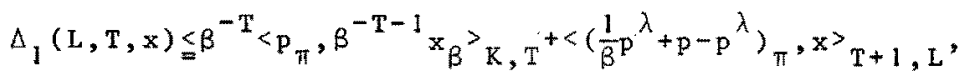

and consequently, (5.5.13) takes the form

$$
B^{\prime} B^{-t} u(t)-B A^{\prime} B^{-t-1} \underset{u}{u}(t+1)-\pi^{t} \delta_{2} e \geq \pi, t p(t), \quad t \geq k .
$$




\subsection{Proposition.}

If the vectors $h \in R^{n}+, g \in 1_{\infty}^{m}$ and $q \in 1_{\infty}^{n}$ are such that the LP-system (5.4.1) is $P$-or D-directed and $P$-and $D$-regular for $x(0)=h$, $f=g$ and $p=q$, then $\left.a \beta^{*} \in\right] 1,1 / \rho \pi[$ exists for which the following holds:

For every $\left.\beta \in] 1, \beta^{*}\right]$, neighbourhoods $\Omega(\mathrm{h}) \mathrm{R}_{+}^{\mathrm{n}}, \Omega(\mathrm{g}) \subset 1_{\infty}^{\mathrm{m}}, \Omega(\mathrm{q}) \subset 1_{\infty}^{\mathrm{n}}$, and positive numbers $N_{1}, N_{2}$ exist such that, for every $x(0) \in \Omega(h)$ and every $(f, p) \in \Omega(g) \times \Omega(q)$ for which the LP-system (5.4.1) is P-or D-directed, the following property holds:

If $x \in 1_{+}^{n}$, for some $z, z_{\beta \pi} \in I_{1^{+}}^{m}$, satisfies:

$$
\left.\begin{array}{rl}
B \times(1) & \leqq \rho f(1)+A x(0)+z(1) \\
B \times(t+1)-A x(t) & \leq \rho{ }^{t+1} f(t+1)+z(t+1), t \geq 1 \\
\left\|x_{B \pi}\right\| ! T^{1}>N_{1}+N_{2}\left\|z_{B \pi}\right\|_{1}, \text { for some } T \geqq 1
\end{array}\right\},
$$

then, an $\varepsilon>0, T^{*} \geqq 1$ and $\underline{x}, \underline{x}_{B \pi} \in 1_{1+}^{n}$, exist satisfying:

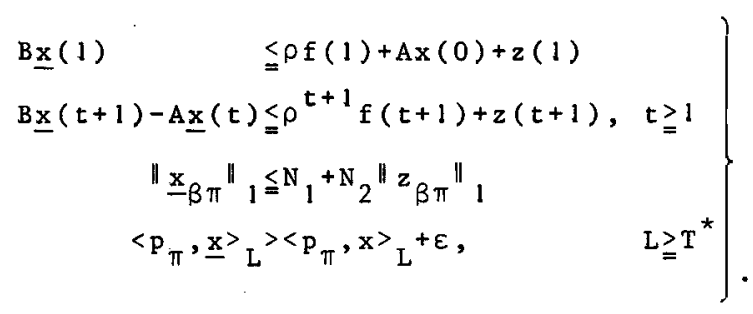

\section{Proof.}

We denote $((\bar{x}, \bar{y}),(\bar{u}, \bar{v}))$ as a P-and D-regular solution of the LP-system (5.4.1) with $x(0)=h, f=g$ and $p=q$. Since $\bar{y}_{1 / \rho} \in \operatorname{int}\left(1_{\infty+}^{\mathrm{m}}\right)$ and $\left.\bar{v}_{1 / \pi} \in \operatorname{int}\left(1_{\infty+}^{n}\right), a \beta^{*} \in\right] 1,1 / \rho \pi[$ exists (close enough to 1) such that for every $\left.\beta \in] 1, \beta^{*}\right]$ property $5.4-b$ holds, with $K=1$, $\tilde{x}:=\beta \bar{x}_{\beta}$, and $\tilde{u}:=\beta \bar{u}_{\beta}$. Hence the conditions of proposition 5.5 . are satisfied for every $\left.\beta \in] 1, \beta^{*}\right]$.

Note $\Omega(\mathrm{h}) \subset \mathrm{R}_{+}^{\mathrm{n}}, \Omega(\mathrm{g}) \subset 1_{\infty}^{\mathrm{m}}$ and $\Omega(\mathrm{q}) \subset 1_{\infty}^{\mathrm{n}}$ as bounded neighbourhoods of $h, g$ and $q$ resp., belonging to certain $\beta \in] 1, \beta^{*}$ ] for which 
the properties 5.5-a,b,c hold. Moreover, we choose these neighbourhoods in such a manner that positive numbers $\delta$, to $\delta_{4}$ exist such that $x:=\beta \bar{x}_{\beta}, \bar{u} ;=\beta \bar{u}_{\beta}, \bar{x}$ and $\bar{u}$ resp. satisfy $(5.4 .3)$ to $(5.4 .6)$ for a $11(x(0), f, p) \in \Omega(h) \times \Omega(g) \times \Omega(q)$.

With the help of the set $\Omega\left(\mathrm{g}^{\circ}\right)$ as defined in $5.4-e$, we put $\Omega\left(g^{\circ}, q\right)$ as the set of vectors $\left(f^{\circ}, p\right) \in \Omega\left(g^{\circ}\right) \times \Omega(q)$ for which LP-system $(5.4 .1)$ is P-or D-directed.

Defining, for every $x \in X\left(\Omega\left(g^{\circ}\right), 1_{+}^{m}\right)$ (def. $5.4-f$ ) and $T \geq K=1$, the vectors $\hat{x}(T, x)$ as in $5.5-h$ with $\hat{x}:=\beta \bar{x}_{\beta}$, then, for all $\left(f^{\circ}, p\right) \in \bar{\Omega}\left(g^{\circ}, q\right), x \in 1_{+}^{n}$ and $z \in 1_{+}^{m}, z_{B \pi} \in 1_{1+}^{m}$ satisfying:

$$
\left.\begin{array}{ll}
B \times(1) & \leq \rho f^{0}(1)+z(1) \\
B x(t+1)-A x(t) \leq p^{t+1} f^{0}(t+1)+z(t+1), t \geq 1
\end{array}\right\}
$$

the following three properties hold:

\section{Property 1}

the vectors $\hat{x}(T, x), T \geq l$ satisfy:

$$
\left.\begin{array}{ll}
B \hat{x}(1 ; T, x) & \leq p f^{0}(1)+z(1) \\
B \hat{x}(t+1 ; T, x)-A \hat{x}(t ; T, x) \leq p t+1 f^{0}(t+1)+z(t+1), t \geq 1
\end{array}\right\} \cdot(5.6 .4)
$$

This follows from $5.5-a$ and from the fact that $\dddot{x}:=\beta \bar{x}_{\beta}$.

\section{Property 2}

$$
\begin{aligned}
& \hat{x}(T, x)_{B \pi} \in 1_{1+}^{n}, \quad T \geq 1, \\
& \left\|\hat{x}(T, x)_{B \pi}\right\| \leq\left\|x_{\beta \pi}\right\| 1, T+\left\|\tilde{x}_{\pi}\right\|, T>1 \\
& \left\|\hat{x}(1, x)_{B \pi 1}\right\| \leq\left\|\tilde{x}_{\pi}\right\|_{1} .
\end{aligned}
$$

This property follows from the definition of $\hat{x}(T, x)(5.4-h)$ and from the fact that $x_{1 / \beta \rho} \in 1_{\infty+}^{n}$ and $\left.\beta \in\right] 1,1 / \rho \pi[$. 


\section{Property 3}

Positive numbers $M_{1}, M_{2}$ and $M_{3}$, which are independent of $x, f^{\circ}, g$ and $z$, exist, such that

$$
\begin{aligned}
& \left\langle\mathrm{p}_{\pi}, \mathrm{x}\right\rangle_{L}-\left\langle\mathrm{p}_{\pi}, \hat{\mathrm{x}}(\mathrm{T}, \mathrm{x})\right\rangle_{\mathrm{L}} \leq \beta^{-T}\left(-\mathrm{M}_{1}\left\|\mathrm{x}_{B \pi}\right\|\left\|_{1}, \mathrm{T+1}+\mathrm{M}_{2}+\mathrm{M}_{3}\right\|_{\beta \pi} z_{1}\right), \\
& \mathrm{T} \geqq 1, \quad \mathrm{~L} \geqq \mathrm{~T}+2
\end{aligned}
$$

This follows from 5.5-c and from the fact that $\beta \in] 1,1 / \rho \pi[$, and that $x(K-1)$ is a vector of the bounded set $\Omega(h)$. Now, define the positive numbers $N_{1}$ and $N_{2}$ by

$$
\left.\begin{array}{l}
\mathrm{N}_{1}:=M 2 / M 1+\left\|\tilde{x}_{\pi}\right\|_{1} \\
N_{2}:=M 3 / M 1
\end{array}\right\}
$$

and suppose that $x \in 1_{+}^{n}$ for certain $\left(f^{\circ}, p\right) \in \bar{\Omega}\left(g^{\circ}, q\right)$ and $z$, $z_{\beta \pi} \in I_{1+}^{\text {Ir. }}$ satisfies $(5.6 .1)$.

Defining for this $x$ a period $\tilde{T}$ by

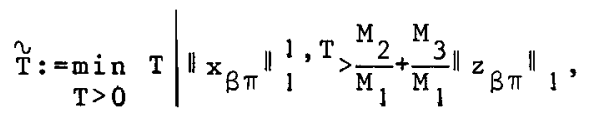

then, from $(5.6 .9),(5.6 .10)$, and from the fact that, for some $T \geqq 1:\left\|x_{\beta \pi}\right\| \begin{aligned} & 1, T_{1} \\ & T N_{1}\end{aligned}+N_{2}\left\|z_{\beta \pi}\right\|$, it follows that $\tilde{T} \geq 1$.

First consider the case that $\tilde{T}=1$. Then, $(5.6 .10)$ and property 3) imply that an $\varepsilon>0$ exists such that

$$
\left\langle\mathrm{p}_{\pi}, \mathrm{x}\right\rangle_{L}-\left\langle\mathrm{p}_{\pi}, \hat{\mathrm{x}}(1, \mathrm{x})\right\rangle_{L} \leq-\varepsilon, \quad L>2 .
$$

property 2) and definition $(5.6 .9)$ imply:

$$
\left.\begin{array}{l}
\hat{x}(1, x)_{\beta \pi} \in 1_{1+}^{n} \\
\left\|\hat{x}(1, x)_{\beta \pi}\right\| \sum_{1} \equiv N_{1}+N_{2}\left\|_{\beta \pi}\right\|_{1}
\end{array}\right\}
$$

From (5.6.11), (5.6.12) and from property 1) we may conclude that $\underline{x}:=\hat{x}(1, x)$ satisfies $(5.6 .2)$ for every $L>2$. 
Now, consider the case that $\tilde{T}>1$. Then, $(5.6 .10)$ and property 3) imply that an $\varepsilon>0$ exists such that

$$
\left\langle\mathrm{p}_{\pi}, x\right\rangle_{L}-\left\langle\mathrm{p}_{\pi}, \hat{x}(\tilde{T}-1, x)\right\rangle_{L} \leqq-\varepsilon, \quad L>\tilde{T}^{2}+1
$$

Property 2) and the definitions $(5.6 .9)$ and $(5.6 .10)$ imply:

$$
\left.\begin{array}{l}
\hat{x}(\tilde{T}-1, x)_{B \pi} \in 1_{1+}^{n} \\
\left\|\hat{x}(\tilde{T}-1, x)_{B \pi}\right\| \sum_{1}^{\leq N}+N_{2}\left\|z_{B \pi}\right\|_{1}
\end{array}\right\} .
$$

From (5.6.13), (5.6.14), and for property 1), we may conclude

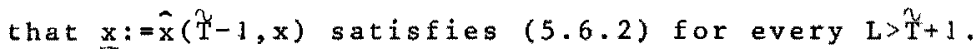

\subsection{Theorem.}

If $h \in \mathrm{R}_{+}^{\mathrm{n}}, g \in 1_{\infty}^{\mathrm{m}}$ and $q \in 1_{\infty}^{\mathrm{n}}$ are such that the LP-system $(5.4 .1)$ is $P$-or D-directed and $P$-and D-regular for $x(0)=h, f=g$ and $p=q$, then a $\left.\beta^{*} \in\right] 1,1 / \rho \pi[$ exists for which the following holds:

For every $\left.\beta \in] 1, \beta^{*}\right]$, neighbourhoods $\Omega(h) \subset_{+}^{n}, \Omega(g) \subset 1_{\infty}^{m}, \Omega(q) \subset 1_{\infty}^{\mathfrak{n}}$ and positive numbers $N_{1}$ and $N_{2}$ exist such that, for every LP-system $(5.4 .1 ; x(0), f, p) \in \Omega(h) \times \Omega(g) \times \Omega(q) ; P$-or D-directed) the following properties hold:

a) For every P-feasible solution $x$ such that

$$
\left\|x_{B \pi}\right\|, T>N_{1}, \quad \text { for some } T \geq 1 \text {, }
$$

a P-feasible $x$ exists, such that $x_{B \pi} \in 1_{1+}^{n},\left\|_{x_{B \pi}}\right\|_{1} \sum_{1}$, and such that $x$ is inferior with respect to $x$.

b) For every D-feasible solution u such that

$$
\left\|u_{B \rho}\right\|,{ }_{1}^{\mathrm{l}}, \mathrm{T}>\mathrm{N}_{2}, \quad \text { for some } \quad \mathrm{T} \gg 1,
$$

a D-feasible solution $\underline{u}$ exists, such that $\underline{u}_{\beta p} \in 1_{1+}^{\mathrm{m}}$, " ${ }_{B \rho} \|, \leqslant N_{2}$, and such that u is inferior with respect to $u$. 


\section{Proof.}

By putting $z=0$, this theorem follows from proposition 5.6 and from the symmetry between the primal and dual systems.

\subsection{Theorem.}

If the vectors $h \in R_{+}^{n}, g \in 1_{\infty}^{m}$, and $q \in 1_{\infty}^{n}$ are such that the LP-system $(5.4 .1)$ is $P$-or $D$-directed and superregular for $x(0)=h$, $f=g$ and $p=q$, then neighbourhoods $\Omega(h) \subset R_{+}^{n}, \Omega(g) \subset 1_{\infty}^{m}, \Omega(q) \subset 1_{\infty}^{n}$ for these vectors, and positive numbers $N_{1}, N_{2}$ exist such that every LP-system (5.4.1; $(x(0), f, p) \in \Omega(h) \times \Omega(g) \times \Omega(q) ; P-o r$ $D$-directed) possesses the following properties:

a) For every P-feasible solution $x$ such that

$$
\left\|\mathrm{x}_{1 / \rho}\right\|_{\infty}^{1, T}>\mathrm{N}_{1}, \quad \text { for some } \quad \mathrm{T} \gg 1,
$$

a P-feasible $x^{*}$ exists, such that $x^{*}{ }_{1 / \rho} \in 1_{\infty+}^{n},\left\|x^{*}{ }_{1 / \rho}\right\|_{\infty} \lesssim N_{1}$, and such that $x$ is inferior with respect to $x^{*}$.

b) For every D-feasible solution u such that

$$
\left\|u_{1 / \pi}\right\|_{\infty}^{1, T}>N_{2}, \quad \text { for some } \quad T \geq 1,
$$

a D-feasible u* exists such that $u^{*^{\prime}},{ }_{1 / \pi} \in 1_{\infty+}^{m},\left\|u^{*}{ }_{1 / \pi}\right\|_{\infty}<N_{2}$, and such that $u$ is inferior with respect to $u^{*}$.

\section{Proof.}

First we shall prove property a).

Since the LP-system (5.4.1) is supposed to be superregular for $(x(0), f, p)=(h, g, q)$, condition $5.4-b$ is satisfied for some $\beta>\frac{1}{\rho \pi}$ (close enough to $\frac{1}{\rho \pi}$ ). Hence, for such a $\beta>\frac{1}{\rho \pi}$ the conditions of proposition 5.5 are satisfied. For the sake of convenience, we put $\mathrm{k}>1$.

Note $\Omega(h) \subset R_{+}^{n}, \Omega(g) \subset 1_{\infty}^{m}$, and $\Omega(q) \subset 1_{\infty}^{n}$ as bounded neighbourhoods of $h, g, q$ resp. for which the properties 5.5-a,b,c hold. Moreover, we choose these neighbourhoods so small that pro- 
perty $5.4-d$ holds, for all $(x(0), f, p) \in \Omega(h) \times \Omega(g) \times \Omega(q)$, and for certain $\delta_{3}, \delta_{4}>0, \bar{x}, \bar{x}_{J / \rho} \in 1_{\infty}^{\mathrm{n}}$ and $\bar{u}, \bar{u}_{1 / \pi} \in 1_{\infty+}^{\mathrm{m}}$.

With the help of the vectors $\hat{x}(T, x), T \geqq K>1$ defined in $5.4-h$, we'now define vectors $\underline{x}(T, x)$ as follows:

$$
\underline{x}(T, x):=\left(1-\alpha_{B}^{-T}\right) \hat{x}(T, x)+\alpha_{B}^{-T} \bar{x}, \quad T \geq S
$$

where $\alpha>0$ is chosen in such a manner that $B \alpha \delta_{3} e \geq B \tilde{x}(\mathrm{~K}) \quad\left(\delta_{3}\right.$ being the quantity of $5.4-d$ ), and period s such that: $\alpha B^{-T}<1, T \geq S$.

So, for every $T \geqq S, \underline{x}(T, x)$ is a convex linear combination of $\hat{x}(T, x)$ and $\bar{x}$, with coefficients $\left(1-\alpha B^{-T}\right), \alpha B^{-T}$ resp. From this observation, from the definition of $x(T, x)$, and from theorem $5.5-b$ (by putting $z=0$ ), we may conclude:

\section{Property 1 .}

For every P-feasible solution $x$ of an LP-system(5.4.1; $(x(0), f, p) \in \Omega(h) \times \Omega(g) \times \Omega(q) ; P$-or $D-d i r e c t e d)$ the vectors $x(T, x), T \geqq S$ are $P-f e a s i b l e$ solutions of this LP-system.

From the definition of $\hat{x}(T, x)$ and from the definition of $\underline{x}(T, X)$, the following property can be derived.

\section{Property 2.}

$$
\begin{aligned}
& x(T, x)_{1 / \rho} \in I_{\infty+}^{n}, \quad T \geq S \\
& \left.\left\|\underline{x}(T, x)_{1 / \rho}^{\|} \leq\right\| x_{1 / \rho}\left\|_{\infty}^{1, T}+\right\| x_{1 / \beta \rho^{2}}\left\|_{\infty}^{+\| \bar{x}_{1 / \rho}}\right\|, T \geq S\right\}
\end{aligned}
$$

Now we shall value down the differences $\left\langle p_{\pi}, x\right\rangle_{L}-\left\langle p_{\pi}, x(T, x)\right\rangle_{L}$, $L>T>S$. For, $z=0$ and $1<1 / \rho \pi<\beta$, the expression $(5.5 .3)$ of proposition 5.5 can be reduced to

$$
\begin{aligned}
& \Delta(L, T, x):=\left\langle p_{\pi}, x\right\rangle_{L}-\left\langle p_{\pi}, \hat{x}(T, x)\right\rangle_{L} \leqq \\
& \leqq(\rho \pi)^{T}\left(-M_{1}\left\|x_{1} / \rho\right\|_{\infty} K, T+1+M_{2}+M_{3}\|x(K-1)\|_{\infty}\right), L>T \gg S,
\end{aligned}
$$


where $M_{1}, M_{2}$ and $M_{3}$ are positive numbers, independent of $x(0)$, $f$ and $p$.

From (5.8.3) and from the definition of the vectors $x(T, x)$, one can derive:

$$
\begin{aligned}
& \left.\Delta(L, T, x):=\left\langle p_{\pi}, x_{L}\right\rangle^{-\left\langle p_{\pi}\right.}, x(T, x)\right\rangle_{L}= \\
& =\left\langle p_{\pi}, x\right\rangle_{L}-\left(1-\alpha \beta^{-T}\right)\left\langle p_{\pi}, x(T, x)\right\rangle_{L}-\alpha \beta^{-T}\left\langle p_{\pi}, x_{L}\right\rangle_{L}= \\
& \left.\left.=\left(1-\alpha \beta^{-T}\right) \Delta(L, T, x)+\alpha \beta^{-T}<p_{\pi}, x\right\rangle_{L}-\alpha \beta^{-T}<p_{\pi}, \bar{x}\right\rangle_{L} \leftrightarrows \\
& \leqq(\rho \pi)^{T}\left[-\left(1-\alpha \beta^{S}\right) M_{1}\left\|_{1 / \rho}\right\|_{\infty}^{K, T+1}+M_{2}+M_{3}\|x(K-1)\|_{\infty}\right]+ \\
& +\alpha \beta^{-T}\left[\left\langle p_{\pi}, x\right\rangle_{L}-\left\langle p_{\pi}, \bar{x}\right\rangle_{L}\right], \quad L>T \geqq S .
\end{aligned}
$$

defining

$\left.M_{4}:=\sup _{T, x(0), f} \times(0)^{\prime} A^{\prime} \bar{u}(1)+\left\langle f f_{0}, \bar{u}\right\rangle_{T} \mid T \geq 1, x(0), f\right) \in \Omega(h) \times \Omega(g)$,

theorem 4.5 implies that

$$
\left\langle\mathrm{P}_{\pi}, \mathrm{x}\right\rangle_{\mathrm{L}} \leq \mathrm{M}_{4}, \quad \mathrm{~L} \gg 1,
$$

for al1 p-feasible solutions-x of the LP-systems $(5.4 .1 ;(x(0), f, p) \in \Omega(h) \times \Omega(g) \times \Omega(q) ; P-o r \quad D-d i r e c t e d)$.

From $(5.8 .4),(5.8 .5)$, and from the fact that $k(1 / \rho \pi)<\beta,\left(1-\alpha \beta^{S}\right)>0$, $\bar{x}_{1 / p} \in 1_{\infty}^{n}$, and $p \in 1_{\infty}^{n}$, we may conclude that positive numbers $M_{5}$, $M_{6}$, and $M_{7}$ exist such that:

\section{Property 3.}

For all p-feasible solutions $x$ of the LP-systems (5.4.1; $(x(0), f, p) \in \Omega(h) \times \Omega(g) \times \Omega(q) ; p$-or $D$-directed), the following inequalities hold:

$$
\triangleq(L, T, x) \leqq(\rho \pi)^{T}\left(-M_{5}\left\|x_{1} / \rho_{\infty}\right\|_{\infty}^{X, T+1}+M_{6}+M_{7}\|x(R-1)\| \infty\right), \quad L>T \geqq S .
$$

Now, we shall demonstrate the validity of the following property: 


\section{Property 4.}

A number $M_{8}>0$ exists such that:

al1 p-feasible solutions $x$ as mentioned in property 3 , with

$$
\left\|\mathrm{x}_{\mathrm{J} / \rho}\right\|_{\infty}^{\mathrm{J}, \mathrm{S}+1}>\mathrm{M}_{8},
$$

are inferior with respect $\bar{x}$.

To prove the validity of this property, we remark that $\bar{x}$ and

$\bar{u}$ are P-and D-regular solutions, for every LP-system (5.4.1; $(x(0), f, q) \in \Omega(h) \times \Omega(g) \times \Omega(q) ; P$-or $p$-directed). Then in a similax manner as theorem 5.2 has been derived, one can conclude that a number $M_{9}$ exists, such that every $P$-feasible $x$ as mentioned in property 3 with

$$
\left\|x_{\pi}\right\| 1, T>M_{9}, \text { for some } T \geq 1
$$

is inferior with respect to $\bar{x}$. Putting $M_{8}:=(1 / 0 \dot{\pi})^{S+1}$ n $M_{g}$, property 4 follows immedeately.

In orderto prove the first part of the theorem, we put

$$
N_{1}:=\frac{1}{M_{5}}\left(M_{6}+\rho^{K-1} M_{7} M_{8}\right)+N_{1 / B \rho}^{n} p_{\infty}+\bar{x}_{1 / \rho} \bar{x}_{\infty}+M_{8}
$$

Now, suppose that $x$ is a p-feasible solution of a certain LP-system $(5.4 .1 ;(x(0), f, p) \in \Omega(h) \times \Omega(g) \times \Omega(q) ; p$-or D-directed) satisfying $(5.8 .1)$ for some $T \geq 1$. We distinguish two cases: $\left\|x_{1 / \rho}\right\|_{\infty}^{1, S+1}>M_{8}$ and $\left\|x_{1 / \rho}\right\|_{\infty}^{1, S+\overline{\overline{1}}} \leqq M_{8}$.

If $\left\|x_{1 / \rho}\right\|_{\infty}^{1, S+1}>M_{8}$, then property 4 implies that $x$ is inferior with respect to $\bar{x}$, which is a p-feasible solution of the LP-system under consideration, and for which (def, 5.8.6):

$$
\left\|\bar{x}_{1 / \rho}\right\| N_{1} \cdot
$$

Hence, for this p-feasible $x$ the theorem has been proven.

If $\left\|x_{1 / \rho}\right\|_{\infty}^{1, S+1} \leq M_{8}$, then define

$$
T^{*}:=\min _{T>1} T \mid\left\|x_{1 / \rho}\right\|_{\infty}^{1, T}>\frac{1}{M_{5}}\left(M_{6}+p^{K-1} M_{7} M_{8}\right)+M_{8} .
$$


Since $\left\|x_{1 / \rho}\right\|_{\infty}^{1, S+1} \leq M_{8}$ the relations $(5.8 .1),(5.8 .6)$, and (5.8.7) imply that $\mathrm{T}^{*}>\mathrm{S}$ and that $\|\mathrm{x}(\mathrm{K}-1)\|_{\infty} \leq \rho{ }^{\mathrm{K}-1} \mathrm{M}_{8}$. From $(5.8 .7)$ and from property 3 , we conclude that an $\varepsilon>0$ exists such that

$$
\left\langle p_{\pi}, x\right\rangle_{L} \leq\left\langle p_{\pi}, \underline{x}\left(T^{*}-1, x\right)\right\rangle_{L}-\varepsilon, \quad L>T^{*}
$$

From $(5.8 .6),(5.8 .7)$, and from property 2 , we may conclude:

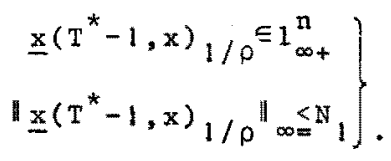

Finally, property 1 implies that $\underline{x}\left(T^{*}-1, x\right)$ is a p-feasibie solution for the LP-system under consideration. Thus, from $(5.8 .8)$ and $(5.8 .9)$ we may conclude that for this p-feasible $x$, too, the theorem is proven.

The second part of the theorem follows from the first part and from the symmetry between the primal and dual systems. 


\section{OPTIMALITX.}

\subsection{Introduction.}

In this chapter we consider the LP-problem(P- or D-directed; Pand $D$-regular; $\rho \pi<1$ ). In that case the theorems 5.2 and 5.3 imply that $\mathrm{P}$-feasible solutions $(x, y)$ and $D$-feasible jolutions $(u, v)$ such that $x_{\pi} \notin 1, y_{\pi}^{n} \notin 1_{1}^{m}$ and $u_{\rho} \notin 1_{1}^{m}, v_{\rho} \notin 11_{1}^{n}$, cannot be optimal. Therefore, we may restrict ourselves to feasible solutions $((x, y),(u, v))$ for which

$$
\begin{aligned}
& x_{\pi} \in 1_{1+}^{n}, y_{\pi} \in 1_{1+}^{m}, \\
& u_{\rho} \in 1_{1+}^{m}, v_{\rho} \in 1_{1+}^{n} .
\end{aligned}
$$

Hence, the LP-problem(P- or D-directed; P- and D-regular;

$\rho \pi<1)$ can be formulated as follows:

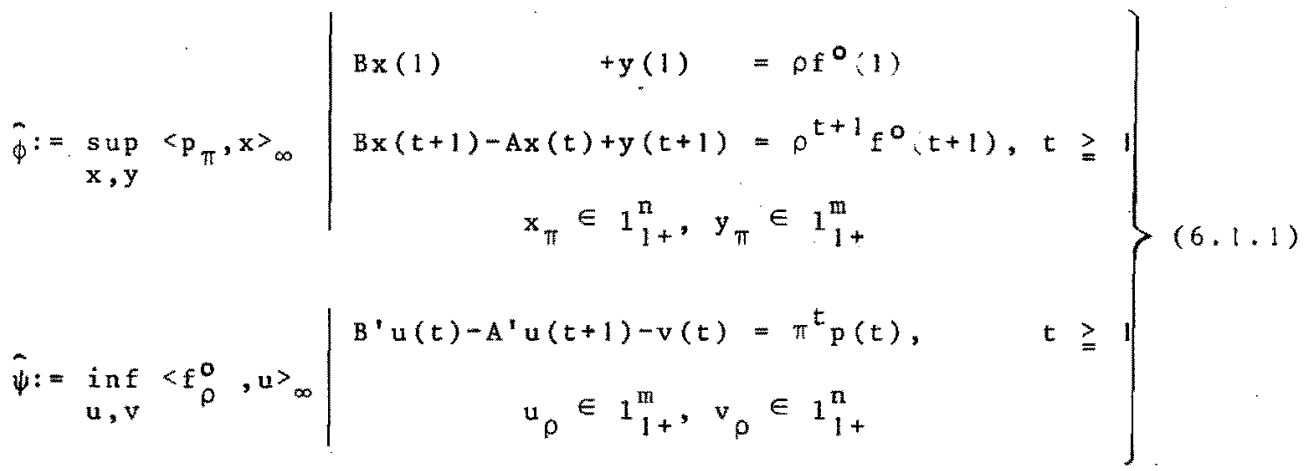

Now, a p-feasible $x$ is called optimal if $\left\langle p_{\pi}, x\right\rangle_{\infty}=\hat{\phi}$, and a D-feasible $u$ is called optimal if $\left\langle f_{p}^{O}, u\right\rangle_{\infty}=\psi$.

The most relevant properties with respect to optimal solutions of this Lp-problem are formulated in the last two theorems of this chapter. 


\subsection{Restatement of the linear programming problem.}

For every scalar $\alpha>0$ we define a matrix $G_{\alpha}$ by:

$$
G_{\alpha}:=\left[\begin{array}{rrrrrr}
B & 0 & 0 & . & . & . \\
-\alpha A & B & 0 & . & . & . \\
0 & -\alpha A & B & & & \\
0 & 0 & : & \cdot & \\
. & . & & . & & \\
. & . & & & & \\
. & . & & & &
\end{array}\right],
$$

With the help of these matrices, the LP-problem $(6.1 .1)$ can be formulated as follows:

$$
\left.\begin{array}{l}
\hat{\phi}:=\sup _{x, y}\left\langle p_{\pi}, x\right\rangle_{\infty} \mid \begin{array}{l}
G_{1} x+y=f_{\rho}^{0} \\
\left(x_{\pi}, y\right) \in y_{1+}^{n} \times 1_{1+}^{m},
\end{array} \\
\hat{\psi}:=\inf \left\langle f_{p}^{o}, u\right\rangle_{\infty} \mid \begin{array}{l}
G_{1}^{\prime} u-v=p_{\pi} \\
\left(u_{\rho}, v_{\rho}\right) \in 1_{1+}^{m} \times 1_{1+}^{n}
\end{array}
\end{array}\right\}
$$

First we shall investigate a more general linear programming

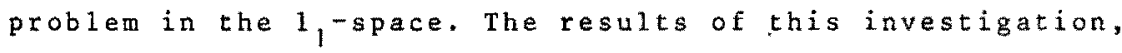
will be transferred later to LP-problem (6.2.2).

\subsection{Definition.}

We define the following linear programming problem:

$$
\hat{\phi:=\sup _{x, y}\langle q, x\rangle_{\infty}} \mid \begin{aligned}
& G x+y=g \\
& x, y \in 1_{1+}
\end{aligned}
$$

where:

a) $q \in 1_{\infty}$ and $g \in 1_{1}$

b) $G$ is a matrix, generating the bounded linear operator $G: 1_{1} \rightarrow 1_{1}$. The adjoint operator is generated by $G^{\prime}$, 
so that, for all vectors $x \in 1_{1}: u^{*} \in 1_{\infty}$ :

$$
\left\langle x, G^{*} u^{*}\right\rangle_{\infty}=\left\langle u^{*}, G\right\rangle_{\infty}
$$

We observe that $\langle q,\rangle_{\infty}$ is a bounded linear functional on $1_{1}$ * $(x, y) \in 1, \times 1$, is called a feasible solution of $(6.3 .1)$, if $x, y \in 1_{1+}$, and $G x+y=g$.

$(\hat{x}, \hat{y}) \in I_{1} \times 1$, is called an optimal solution of $(6.3 .1)$, if $(\hat{x}, \hat{y})$, is a feasible solution of $(6,3,1)$ for which $\hat{\phi}=\langle q, x\rangle_{\infty}$ ( $\Phi$ being the supremum appearing in $(6.3 .1)$ ).

With respect to the analysis of problem $(6.3 .1)$, we remark that the so called generalized Kuhn-Tucker theorem (5:249) is not. applicable. This is due to the fact that the interior of $1_{1+}$ is empty. For this reason we follow the treatment of van slyke and Wets (10), where, with the help of set

$$
\Gamma:=\left\{(\phi, z) \in \mathbb{R}^{1} \times 1, \mid \begin{array}{r}
\phi \leqq\langle, x\rangle_{\infty}, z=G x+y-g, \\
\text { for some } x, y \in 1,
\end{array}\right\},(6.3 .3)
$$

the following programming problem is joined to $(6.3 .1)$ :

$$
\sup _{(\phi, z)} \mid(\phi, z) \in \Gamma \cap\left(R^{\prime} \times(0)\right) .
$$

Clearly, set $\Gamma$ possesses the following properties (see fig.1):

c) $\Gamma$ is convex,

d) for every $z_{1}, z_{2} \in I_{1}$, such that $z_{1} \geq z_{2}$ :

$$
\left\{\phi \mid\left(\phi, z_{2}\right) \in \Gamma\right\} \subset\left\{\phi \mid\left(\phi, z_{1}\right) \in \Gamma\right\},
$$

e) $R^{\prime} \times\{0\}$ is closed.

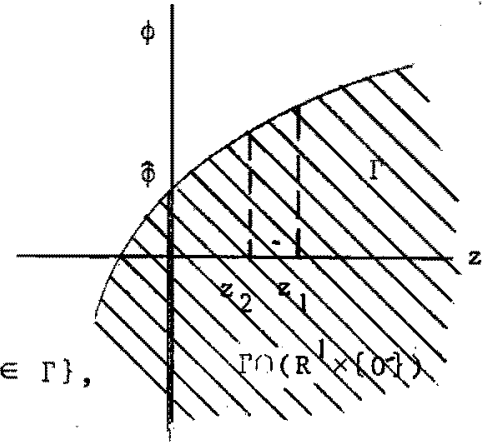

Fig: 1

A point $(\phi, z) \in\left(R^{1} \times 1_{1}\right)$ is called a feasible solution of (6.3.4) if $(\phi, z) \in \Gamma \cap\left(R^{\prime} \times\{0\}\right)$ and an optimal solution if, in addition, $\phi$ is equal to the supremum in $(6.3 .4)$. 
Clearly, the definitions of the problems $(6.3 .1)$ and $(6.3 .4)$ imply the following proposition:

\subsection{Proposition.}

a) Problem (6.3.1) possesses a feasible solution, if and only if $T \cap\left(R^{1} \times\{0\}\right) \neq 0$.

b) The supremum in $(6.3 .1)$ is equal to the supremum in $(6.3 .4)$.

c) Problem (6.3.1) possesses an optimal solution, if and only if problem $(6.3 .4)$ possesses an optimal solution.

d) Problem (6.3.4) possesses an optimal solution, if and only if the supremum in $(6.3 .4)$ is bounded and moreover the set I $\cap\left(R^{1} \times\{0\}\right)$ is non-empty and closed.

We see that it is sensible first to analyse the joined problem $(6.3 .4)$. In that analysis the concept of dual space, now to be introduced, takes a central place.

\subsection{Dual spaces $(*)$}

The set of bounded linear functionals $f(x)$ on a Banach space $x$ can, by introducing the vector sum and scalar multiplication, be taken as a vector space. If to this vector space the following norm is joined:

$$
\|f(x)\|_{x^{*}}:=\sup _{x}|f(x)| \mid x \in x,\|x\| x \leqq 1,
$$

where $\| \mathrm{X}$ is the norm of the Banach space $x$ under consideration, then this new vector space is called the normed dual space of $x$ or, briefly, the dual space of $x$. The common notation for this space is $X^{*}$. Now, we wish to mention some properties of dua 1 spaces.

(*) For a more detailed treatment see for instance Luenberger(5). 
Every bounded linear functional $f(x)$ on the 1 , space can be represented uniquely by a vector $v \in l_{\infty}$ in the following manner:

$$
f(x)=\langle v, x\rangle_{\infty}:=\sum_{i=1}^{\infty} v_{i} x_{i} .
$$

The norm of $1_{1}^{*}$ is exactly the $1_{\infty}$-norm. So, the $1_{\infty}$-space may be taken as the dual space of 1 ,

The $c_{0}$-space is defined as the set of sequences of numbers in $R^{\prime}$ converging to zero. This space is equipped with the $1_{\infty}$-norm. Thus, $c_{0}$ is a subspace of $1_{\infty}$. In a similar manner as explained for $1_{1}^{*}$, the $1,-s p a c e$ may be taken as $c_{0}^{*}$.

The dual space of $1_{\infty}$ is not $1_{1}$. However, every $v \in 1_{1}$ represents a bounded linear functional on $1_{\infty}$ by $\langle v, x\rangle_{\infty}$.

Now we sha11 introduce the concepts weak ${ }^{*}$ convergence, weak compactness, and weak* closedness, which are all strongly related to the concept of dual space.

Let $X^{*}$ be the dual space of a Banach space $X$, then we say that a sequence $\left\{f_{i}\right\}_{1}^{\infty} \subset X^{*}$ converges weak* to $f_{o} \in X^{*}$ if for every $\mathrm{x} \in \mathrm{X}:$

$$
f_{i}(x) \rightarrow f_{0}(x), \quad i \rightarrow \infty .
$$

In this study we shall denote weak ${ }^{*}$ convergence of a sequence

$$
\begin{aligned}
\left\{\mathrm{f}_{i}\right\}_{1}^{\infty} \subset \mathrm{x}^{*} \text { to } \mathrm{f}_{0}, \text { by } & \\
\mathrm{f}_{i} & \stackrel{\sim}{ } \mathrm{f}_{\mathrm{o}}, \mathrm{i} \rightarrow \infty .
\end{aligned}
$$

Since $c_{0}^{*}=1_{1}$ and $I_{1}^{*}=1_{\infty}$, weak convergence $(6.5 .4)$ of a sequence $\left\{f_{i}\right\}_{1}^{\infty}$ in 1 , or $1_{\infty}$ implies

$$
\left\langle E_{i}, x\right\rangle_{\infty} \rightarrow\left\langle E_{0}, x\right\rangle_{\infty}, i \rightarrow \infty \text {, }
$$

for every $x \in c_{0}$ or $x \in l_{\infty}$ resp.

The concepts of convergence and weak ${ }^{*}$ convergence are further related by: 
- convergence implies weak* convergence,

- for finite dimensional spaces the concepts are equivalent.

A set $z \subset x^{*}$ is called weak ${ }^{*}$ compact, when every sequence $\left\{f_{i}\right\}_{1}^{\infty} C z$ contains a subsequence $\left\{f_{i}(k)\right\}_{k=1}^{\infty}$ which converges weak* to a vector $f_{0} \in Z$.

A set $Z \subset X^{*}$ is called weak* closed, when every weak ${ }^{*}$ convergent sequence $\left\{f_{i}\right\}_{1}^{\infty} \subset x^{*}$, converges weak to a point in $z$.

Further, we remark that $1_{1+}$ and $1_{\infty+}$ are both weak* closed, and so, since convergence implies weak* convergence, closed as we 11 .

\subsection{Definition.}

To $(6.3 .4)$, we join the following problem:

$$
\begin{array}{l|l}
\text { in }{ }^{*} \psi & \left(\psi, u^{*}\right) \in\left(R^{1} \times 1_{\infty}\right) \\
u^{*} \psi & \left\langle u^{*}, z_{\infty}-\phi+\psi \geqslant 0, \text { for all }(\phi, z) \in \Gamma .\right.
\end{array}
$$

Since $\left(R^{1} \times 1_{1}\right)^{*}=\left(R^{1} \times 1_{\infty}\right)$, for every $\left(r^{*}, u^{*}\right) \in\left(R^{1} \times 1_{\infty}\right)$ and $\psi \in R^{\prime}$, the expression

$$
n^{*} \phi+\left\langle u^{*}, z\right\rangle_{\infty}+\psi=0
$$

may be interpreted as a closed hyperplane in $\left(R^{1} \times 1_{1}\right)$.

Figure 2 gives such a hyperplane, in case $\eta^{*}=1$ and $\left(\psi, u^{*}\right)$ satisfies the inequa $1-$ ity of $(6.6 .1)$ for all

$(\phi, z) \in \Gamma$.

Hence, problem $(6.6 .1)$ may be considered as a process of seeking a supporting hyperplane $(6.6 .2)$

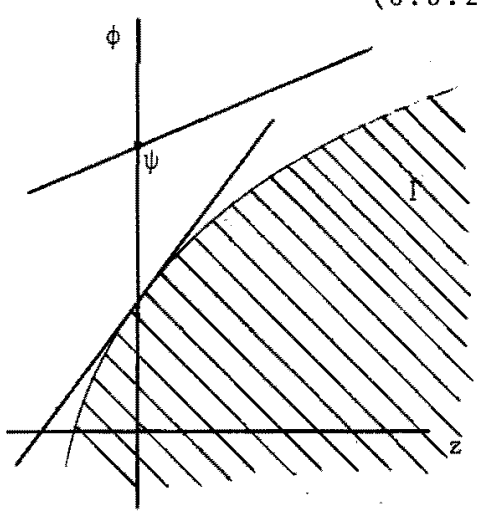

Fig. 2 
such that

$-n^{*}=-1$ (i.e. non-vertical),

- the vertical axis is intersected at a point as low as possible.

We introduce the following terms:

- $\left(\psi, u^{*}\right) \in\left(R^{\prime} \times 1_{\infty}\right)$ is called a feasible solution of $(6.6 .1)$, if $\left(\psi, u^{*}\right)$ satisfies the inequality of $(6.6 .1)$ for all $(\phi, z) \in \Gamma$.

- $\left(\psi, u^{*}\right) \in\left(R^{1} \times 1_{\infty}\right)$ is called an optimal solution of (6.6.1), if $\left(\psi, u^{*}\right)$ is feasible and $\psi$ is equal to the infinum in $(6,6,1)$.

The geometric interpretation of $(6.6 .1)$ suggests that the supremum in (6.3.4) cannot be higher than the infinum in (6.6.1) and that, generally, the supremum in (6.3.4) will be equal to the infinum in $(6.6,1)$. The next two propositions will affirm these presumptions.

\subsection{Proposition.}

If the problems $(6.3 .4)$ and $(6.6 .1)$ both possess a feasible solution, then the infinum in $(6.6 .1)$ is not smaller than the supremum in $(6,3.4)$. Moreover, in that case the infinum of $(6.6 .1)$ and the supremum of $(6.3 .4)$ are both finite.

\section{proof.}

The definitions of $(6.3 .4)$ and $(6.6 .1)$ imply

$$
\left\langle\mathbf{u}^{*}, \mathbf{z}\right\rangle_{\infty}+\psi \geqq \phi
$$

for all feasible solutions $(\phi, z)$ and $\left(\psi, u^{*}\right)$ of $(6.3 .4)$ and (6.6.1) resp. Putting $z=0$, we have $\psi \geqq \phi$ for all feasible $(\phi, z)$ and $\left(\psi, u^{*}\right)$.

The second part of the proposition immediately follows from $(6.7 .1)$. 


\subsection{Proposition.}

If the problems $(6.3 .4)$ and $(6.6 .1)$ both possess a feasible solution, and if

$$
\overline{\Gamma \cap\left(R^{1} \times\{0\}\right)}=\bar{\Gamma} \cap\left(R^{1} \times\{0\}\right),
$$

then the supremum in $(6.3 .4)$ is equal to the infinum in $(6,6,1)$.

\section{Proof.}

Defining $\hat{\phi}$ as the supremum in $(6.3 .4)$, definition $(6.3 .4)$ and supposition $(6.8 .1)$ imply

$$
\{\phi \in R ! \mid \phi \leqq \bar{\phi}\} \times\{0\}=\overline{\Gamma \cap\left(R^{1} \times\{0\}\right)}=\bar{\Gamma} \cap\left(R^{1} \times\{0\}\right) .
$$

$(6.8 .2)$

Since $\hat{\phi}$ is finite (proposition 6.7 ), for every $\varepsilon>0$ :

$$
\hat{(\phi+\varepsilon, 0) \notin \Gamma}
$$

Since $\bar{\Gamma}$ is a closed convex $(6.3-c)$ set in $\left(R^{1} \times 1_{1}\right)$, it follows from (6.8.3) that, for every $\varepsilon>0$, a closed halfspace $I_{\varepsilon} \subset\left(R^{!} \times I_{1}\right)$ exists $(5: 134)$ for which:

$$
\left.\begin{array}{lll}
\bar{\Gamma} \subset \hat{\Gamma}_{\varepsilon} & \\
& & \\
(\phi+\varepsilon, 0) & \ddagger & \hat{\Gamma}_{\varepsilon}
\end{array}\right\} .
$$

Since $\left(R^{1} \times 1_{1}\right)^{*}=\left(R^{1} \times 1_{\infty}\right)$, every halfspace ${ }^{*}$ can be expressed by

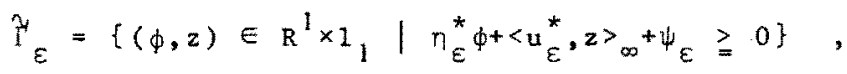

where $\left(n_{\varepsilon}^{*}, u_{\varepsilon}^{*}\right) \in\left(R^{1} \times 1_{\infty}\right)$ and $\psi_{\varepsilon} \in R^{1}$, further to be determined. From $(6.8 .4)$ and $(6.8 .5)$ we may conclude that $\eta_{\varepsilon}^{*}<0$; for if $\eta_{\varepsilon}^{*} \geqslant 0$, then $(6.8 .5)$ implies that not simultaneously $(\hat{\phi}, 0) \in \hat{\mathbb{I}}_{\varepsilon}$ and $(\hat{\phi}+\varepsilon, 0) \notin \hat{I}_{\varepsilon}$; however, this is impossible on account of $(6.8 .4)$. 
Since $\eta_{\varepsilon}^{*}<0$ and since the expression $\eta_{\varepsilon}^{*} \phi+\left\langle u_{\varepsilon}^{*}, z\right\rangle_{\infty}+\psi_{\varepsilon}$ is homogeneous in $\eta_{\varepsilon}^{*}$, ${ }^{*}$ and $\psi_{\varepsilon}$, we may choose these quantities in such a manner that $\eta_{\varepsilon}^{*}=-1$. Thus, we may conclude: for every $\varepsilon>0$, a $\left(\psi_{\varepsilon}, u_{\varepsilon}^{*}\right) \stackrel{\varepsilon}{\epsilon}\left(R^{\prime} \times 1_{\infty}\right)$ exists, such that the set

$$
Y_{\varepsilon}:=\left\{(\phi, z) \in R^{\prime} \times 1, \mid\left\langle u_{\varepsilon}^{*}, z\right\rangle_{\infty}-\phi+\psi_{\varepsilon} \geq 0\right.
$$

satisfies $(6.8 .4)$. This implies, for every $\varepsilon>0$, the existence of a $\left(\psi_{\varepsilon}, u_{\varepsilon}^{*}\right) \in\left(R^{1} \times 1_{\infty}\right)$ such that:

$$
\begin{aligned}
\left\langle\mathrm{u}_{\varepsilon}^{*}, \mathrm{z}\right\rangle-\phi+\psi_{\varepsilon} & \geqq 0, \text { for all }(\phi, z) \in \Gamma \\
\psi_{\varepsilon} & \leqq \hat{\Phi}+\varepsilon
\end{aligned}
$$

From $(6.8 .7)$ it appears that $\left(\psi_{\varepsilon}, u_{\varepsilon}^{*}\right)$ is a feasible solution of $(6.6 .1)$, so that by virtue of proposition 6.7 and $(6.8 .7)$ :

$$
\hat{\phi} \leqq \psi_{\epsilon} \leqq \hat{\phi}+\varepsilon \text {. }
$$

This implies $\psi_{\varepsilon} \rightarrow \hat{\phi}, \varepsilon \rightarrow 0$, and thus the equality of the supremum in $(6.3 .4)$ and the infimum in $(6.6 .1)$.

\subsection{Example.}

We shall illustrate the meaning of condition (6.8.1) with the help of the following programing problem:

$$
\sup \sum_{i=1}^{\infty} x_{i} \mid \begin{aligned}
& \sum_{i=1}^{\infty} x_{i} \leqq 1+z_{1} \\
& \sum_{i=1}^{\infty}\left(\frac{1}{2}\right)^{i} x_{i} \leqq 0+z_{2} \\
& x_{i} \geqq 0, i=1,2, \ldots .
\end{aligned}
$$

This example can be taken as a particular case of problem $(6,3,1)$.

Putting $\left(z_{1}, z_{2}\right)=(0, \varepsilon)$, it appears:

- there is no feasible solution for $E<0$,

- for $\varepsilon=0 ; x_{i}=0, i \geqq 1$ is the only feasible solution; 
so, the supremum in $(6.9 .1)$ is zero.

- for $\varepsilon>0$, the supremum in $(6.9 .1)$ is one.

Figure 3 gives a sketch of the set

$\Gamma_{2}:=\Gamma \cap\left\{\left(\phi, z_{1}, z_{2}\right) \in R^{3} \mid z_{1}=0\right\}$.

obviously, for this problem:

$\overline{\Gamma \cap\left(R^{1} \times\{0\}\right)} \neq \bar{\Gamma} \cap\left(R^{1} \times(0\}\right)$.

One may deduce that, for $\left(z_{1}, z_{2}\right)=(0,0)$, the corresponding problem $(6.6 .1)$ has an optimal solution $\left(\psi, u_{1}^{*}, u_{2}^{*}\right)=(1,1,1)$.

Thus, the supremum in $(6.9 .1)$ for $\left(z_{1}, z_{2}\right)=$ $(0,0)$ is strictly smaller than the infinum of the corresponding problem $(6.6 .1)$. This property is known as the so called "duality gap".

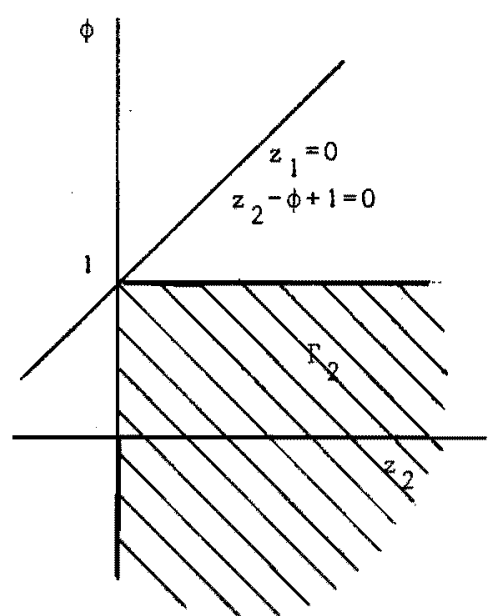

Fig. 3

\subsection{Definition.}

We define the linear programing problem:

$$
\hat{\psi}:=\inf _{u^{*}, v^{*}}^{\left\langle g, u^{*}\right\rangle} \mid \begin{aligned}
& G^{\prime} u^{*}-v^{*}=q \\
& u^{*}, v^{*} \in 1_{\infty+}
\end{aligned}
$$

where:g $\in 1, q \cong 1_{\infty}$ and $G$ are the same quantities as introduced for problem $(6,3,1)$.

We observe that this problem is formulated in $1_{\infty}$, which is the dual space of the space in which problem (6.3.1) is formulated. The nomenclature with respect to the concept. of duality as introduced in $\$ 2.5$, is based on this consideration, and on the relations between $(6.3 .1)$ and $(6.10 .1)$, further to be deduced.

First we shall give some relations between the problems $(6.6 .1)$ and $(6.10 .1)$.

\subsection{Proposition.}

$\left(\psi, u^{*}\right) \in\left(\mathrm{R}^{1} \times 1_{\infty}\right)$ is a feasible solution of $(6,6.1)$, if and on $1 \mathrm{y}$ 
if $\left(\psi, u^{*}\right)$ possesses the following properties:

$$
\left.\begin{array}{rl}
\left\langle g, u^{*}\right\rangle_{\infty} & \leqq \psi \\
u^{*} & \in I_{\infty+} \\
v^{*}:=G^{\prime} u^{*}-q \in I_{\infty+}
\end{array}\right\} .
$$

Proof.

The definition of problem (6.6.1), the definition (6.3.3) of $\Gamma$ and supposition $6.3-b$ imply successively the equivalence of the following statements:

$$
\begin{aligned}
& \left(\psi, u^{*}\right) \in\left(R^{\prime} \times 1_{\infty}\right) \text { is a feasible solution of }(6.6 .1) \text {, } \\
& \langle u *, z\rangle_{\infty}-\phi+\psi \geqslant 0 \text {, for a } 11(\phi, z) \in \Gamma \text {, } \\
& \left.\left\langle u^{*}, G x+y-g\right\rangle_{\infty}-\langle q, x\rangle_{\infty}+\psi\right\rangle 0 \text {, for al1 } x, y \in 1_{1+}, \\
& \left\langle G^{\prime} u^{*}-q, x\right\rangle_{\infty}+\left\langle u^{*}, y\right\rangle_{\infty}-\left\langle g, u^{*}\right\rangle_{\infty}+\psi \geqq 0 \text {, for } a 11 x, y, \in I_{1+} \text {. }
\end{aligned}
$$

Let $\left(\psi, u^{*}\right)$ be a feasible solution of $(6.6 .1)$, then the necessity of the conditions $(6.11 .1)$ can be proved as follows:

- Putting $x:=0, y:=0$, equality $(6.11 .2)$ implies:

$$
\left\langle\mathrm{g}, \mathrm{u}^{*}\right\rangle_{\infty} \leqq \psi
$$

- Putting $x:=0$, equality $(6.11 .2)$ implies:

$$
\left\langle u^{*}, y\right\rangle_{\infty}-\left\langle g, u^{*}\right\rangle_{\infty}+\psi \geqq 0, \text { for a } 11 \text { y } \in 1_{1+} \text {. }
$$

This is possible only when $u^{*} \in 1_{\infty+}$; for, $u^{*} \notin 1_{\infty+}$ implies the existence of a $y \in 1_{\infty+}$ such that the left hand side of (6.11.3) is negative.

- Defining $v^{*}:=G^{\prime} u^{*}-q$ and putting $y:=0$, equality $(6,11,2)$ implies:

$$
\left\langle v^{*}, x\right\rangle_{\infty}-\left\langle g, u^{*}\right\rangle_{\infty}+\psi \geqslant 0, \text { for a } 11 x \in 1_{1+} \text {. }
$$

This is possible on $1 y$ if $v^{*} \in l_{\infty+}$. 
Thus it appears that the conditions $(6.11 .1)$ are necessary. The sufficiency of the conditions $(6.11 .1)$ immediately follows from $(6.11 .2)$, because $(6.11 .1)$ implies:

$$
\left.\begin{array}{rl}
-\left\langle g, u^{*}\right\rangle_{\infty}+\psi & \geqq 0 \\
\left\langle G^{\prime} u^{*}-q, x\right\rangle_{\infty} & \geq 0, \text { for all } x \in 1_{1+} \\
\left\langle u^{*}, y\right\rangle_{\infty} & \geqq 0, \text { for all y } \in 1_{1+}
\end{array}\right\}
$$

and so the validity of $(6.11 .2)$ for all $x, y \in 1_{1+}$.

\subsection{Corollary.}

a) Problem $(6.10 .1)$ possesses a feasible solution, if and only if problem $(6.6 .1)$ possesses a feasible solution.

b) The infinum in $(6.10 .1)$ is equal to the infinum in $(6.6 .1)$.

c) Problem (6.10.1) possesses an optimal solution, if and only if problem (6.6.1) possesses an optimal solution.

\subsection{Proposition.}

If the problems $(6.3 .1)$ and $(6.10 .1)$ both possess a feasible solution, then:

a) $\hat{\phi} \leqq \hat{\psi}$

b) For every feasible solution $(x, y),(u, v)$ of $(6.3 .1),(6.10 .1)$ resp. the following equality holds:

$$
\langle q, x\rangle_{\infty}=\left\langle g, u^{*}\right\rangle_{\infty}-\left\langle v^{*}, x\right\rangle_{\infty}-\left\langle u^{*}, y\right\rangle_{\infty}
$$

c) If the set $\Gamma$ (def 6.3 .3$)$ satisfies

$$
\overline{\Gamma \cap\left(R^{1} \times\{0\}\right)}=\bar{\Gamma} \cap\left(R^{1} \times\{0\}\right),
$$

then

$$
\hat{\phi}=\hat{\psi}
$$

d) If the set $\Gamma$ satisfies $(6.13 .1)$, then feasible solutions 
$(x, y),(u, v)$ of $(6.3 .1),(6.10 .1)$ resp. are both optimal if and on $1 \mathrm{y}$ if

$$
\left\langle v^{*}, x\right\rangle_{\infty}+\left\langle u^{*}, y\right\rangle_{\infty}=0
$$

Proof.

(a) This property immediately follows from the propositions $6.4,6.7$ and 6.12 .

(b) Let $(x, y)$ and $(u, v)$ be feasible solutions of (6.3.1) and (6.10.1) resp. Then, $6.3-b$ and the definition of the problems $(6.3 .1),(6.10 .1)$ imply:

$$
\begin{aligned}
& \langle q, x\rangle_{\infty}=\left\langle G^{\prime} u^{*}-v^{*}, x\right\rangle_{\infty}=\left\langle G^{\prime} u^{*}, x\right\rangle_{\infty}-\left\langle v^{*}, x\right\rangle_{\infty}= \\
& =\left\langle u^{*}, G x\right\rangle_{\infty}-\left\langle v^{*}, x\right\rangle_{\infty}=\left\langle u^{*}, g-y\right\rangle_{\infty}-\left\langle v^{*}, x\right\rangle_{\infty}= \\
& =\left\langle\mathrm{g}, \mathrm{u}^{*}\right\rangle_{\infty}-\left\langle\mathrm{u}^{*}, \mathrm{y}\right\rangle_{\infty}-\left\langle\mathrm{v}^{*}, \mathrm{x}\right\rangle_{\infty} .
\end{aligned}
$$

(c) The validity of this property follows from the propositions $6.4,6.7,6.8$ and 6.12 .

(d) This property is implied by b) and c).

\subsection{Remark.}

In the following proposition conditions are given, implying the existence of an optimal solution of $(6.3 .1)$ and the validity of the equality

$$
\overline{\Gamma \cap\left(R^{l} \times(0\}\right)}=\bar{\Gamma} \cap\left(R^{1} \times\{0\}\right) \text {. }
$$

Later, it will appear that an LP-problem(P-or D-directed; P- and D-regular; $\rho \pi<1)$ satisfies these conditions.

\subsection{Proposition.}

If the problems $(6.3 .1)$ and $(6.10 .1)$ are such that:

a) The conditions $6.3-a, b$ are satisfied. 
b) $\mathrm{q} \in \mathrm{c}_{\mathrm{o}}$.

c) Matrix $G^{\prime}$ generates a bounded linear operator $G^{\prime}: c_{0} \rightarrow c_{0}$.

d) Problem $(6,3,1)$ possesses a feasible solution.

e) Numbers $M_{1}, M_{2}$ exist such that, for every $(\phi, z) \in \Gamma \cap\left(R^{\prime} \times 1_{1+}\right)$, the system

$$
\left.\begin{array}{c}
G \mathrm{x} \leqq \mathrm{g}+\mathrm{z} \\
\|\mathrm{x}\|_{1} \leqq \mathrm{M}_{1}+\mathrm{M}_{2}\left\|_{\mathrm{z}}\right\|_{1} \\
\langle\mathrm{q}, \mathrm{x}\rangle_{\infty} \geqq \phi \\
\text { possesses a solution } \mathrm{x} \in \mathrm{I}_{1++^{\circ}}
\end{array}\right\}
$$

Then, problem (6.3.1) possesses an optimal solution, and the equality

$$
\overline{\Gamma \cap\left(R^{\prime} \times\{0\}\right)}=\bar{\Gamma} \cap\left(R^{\prime} \times\{0\}\right)
$$

holds.

\section{Proof.}

Consider a sequence:

$$
\begin{aligned}
& \left\{\left(\phi_{i}, z_{i}\right)\right\}_{1}^{\infty} \subset \Gamma \cap\left(R^{l} \times 1_{1}\right) \\
& \left(\phi_{i}, z_{i}\right) \rightarrow\left(\phi_{0}, z_{0}\right), i \rightarrow \infty .
\end{aligned}
$$

The existence of such a sequence is implied by d), for $\Gamma \cap\left(R^{1} \times\{0\}\right) \neq \emptyset$ (proposition 6.4-a).

Supposition e) implies the existence of a sequence $\left\{x_{i}\right\}_{1}^{\infty} \subset 1_{1+}$ satisfying

$$
\left.\begin{array}{l}
G x_{i} \leqq g^{+z_{i}} \\
\left\|x_{i}\right\| \leqq M_{1}+M_{2}\left\|z_{i}\right\| \\
\left.<q, x_{i}\right\rangle_{\infty} \geqq \phi_{i}
\end{array}\right\} \quad i \geqq 1 .
$$


If we define the set

$$
0:=\left\{x \in 1_{1} \mid\|x\|_{1} \leqq M_{1}+M_{2} \sup _{i \geqq 1}\left\|z_{i}\right\|_{1}\right\},
$$

then $(6.15 .5)$ implies

$$
\left\{x_{i}\right\}_{1}^{\infty} \subset \sigma
$$

Since $\sigma$ is wak* compact (Aloaglu's theorem $(1: 272)$ ), (6.15.6) implies the existence of a subsequence $\left\{x_{i(k)}\right\}_{k=1}^{\infty}$ of $\left\{x_{i}\right\}_{1}^{\infty}$ which converges weak ${ }^{*}$ to a point $x_{0} \in \sigma$, i.e.:

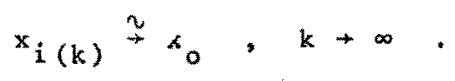

Supposition b) and weak * convergence $(6.15 .7)$ imply:

$$
\left\langle q, x_{i(k)}\right\rangle_{\infty} \rightarrow\left\langle q, x_{0}\right\rangle_{\infty}, k \rightarrow \infty .
$$

The relations $(6.15 .4),(6.15 .5)$, and $(6.15 .8)$ imply:

$$
\left\langle q, x_{0}\right\rangle_{\infty} \geq \phi_{0}
$$

The suppositions a) and $c$ ) imply for every $r \in c_{0}$ :

$$
\left.\begin{array}{l}
G^{\prime} r \in c_{0} \\
\left\langle r, G x_{i}\right\rangle_{\infty}=\left\langle G^{\prime} x^{i}, x_{i}\right\rangle_{\infty}, i \geqq 0
\end{array}\right\},
$$

and so, in connection with $(6.15 .7)$ :

$$
\left\langle\mathbf{r}, \mathrm{Gx}_{i(\mathrm{k})}\right\rangle_{\infty} \rightarrow\left\langle\mathrm{r}, \mathrm{Gx}_{0}\right\rangle_{\infty}, \mathrm{k} \rightarrow \infty \text {, for al1 } \mathrm{r} \in \mathrm{c}_{0}
$$

From $(6.15 .4),(6.15 .5)$, and $(6.15 .10)$, we may conclude:

$$
G \mathbf{x}_{0} \leqq g+z_{0}
$$




$$
\begin{aligned}
& \text { Since } 1_{1+} \text { is weak* closed, }(6.15 .3),(6.15 .4),(6.15 .7) \text {, and } \\
& \left\{x_{i(k)}\right\}_{k=1}^{\infty} \in 1_{1+} \text { imply } \\
& z_{0}, x_{0} \in 1_{1+} \text {. }
\end{aligned}
$$

The definition (6.3.2) of $\Gamma$ and the relations $(6.15 .9),(6.15 .11)$, $(6.15 .12)$ imply $\left(\phi_{0}, z_{0}\right) \in \Gamma \cap\left(R^{1} \times I_{1+}\right)$. Thus, we find that every convergent sequence $\left\{\left(\phi_{i}, z_{i}\right)\right\}_{1}^{\infty} \subset \Gamma \cap\left(R^{l} \times 1_{1+}\right)$ convergès to a point in $T \cap\left(R^{l} \times 11_{1}\right)$. Hence:

$$
\Gamma \cap\left(R^{1} \times 1_{1+}\right)=\overline{\Gamma n\left(R^{1} \times 1_{1+}\right)} \text {. }
$$

With the help of this result the proof will be completed as follows.

Since the set $\left(R^{1} \times[0\}\right)$ is closed, $(6.15 .13)$ implies:

$$
\Gamma \cap\left(\mathrm{R}^{1} \times(0\}\right)=\overline{\Gamma \cap\left(\mathrm{R}^{\prime} \times\{0\}\right)} .
$$

Supposition e) implies the boundness of the supremum in (6.3.4), and so, by virtue of $(6.15 .14)$ and proposition 6.4 , we may conclude: problem $(6.3 .1)$ possesses an optimal solution.

The validity of $(6.15 .2)$ may be proved as follows.

Equality $\Gamma=\left(\Gamma \cap\left(R^{\prime} \times 1_{1-}\right)\right) \cup\left(\Gamma \cap\left(R^{\prime} \times 1_{1+}\right)\right.$ implies successive$1 \mathrm{y}$

$$
\vec{\Gamma}=\overline{\left(\Gamma \cap\left(R^{1} \times 1_{1-}\right)\right)} \cup \overline{\left(\Gamma \cap\left(R^{l} \times 1_{1+}\right)\right)},
$$

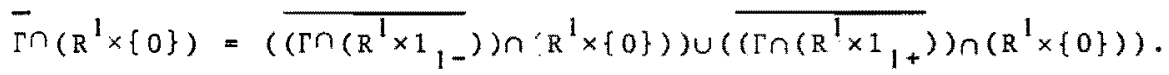

Property 6.3-d implies:

$$
\overline{\left(\Gamma \cap\left(R^{1} \times 1-1\right)\right.} \cap\left(R^{1} \times(0)\right) \subset \overline{\left(\left(\Gamma \cap\left(R^{1} \times 1_{1+}\right)\right)\right.} \cap\left(R^{1} \times[0\}\right) .
$$


With the help of $(6.15 .15),(6.15 .16),(6.15 .13)$, and $(6.15 .14)$, we derive:

$$
\begin{aligned}
\bar{\Gamma} \cap\left(R^{1} \times\{0\}\right) & =\overline{\left(\left(\Gamma \cap\left(R^{\prime} \times 1,1+\right)\right.\right.} \cap\left(R^{\prime} \times\{0\}\right)= \\
& =\Gamma n\left(R^{1} \times\{0\}\right)=\overline{\Gamma \cap\left(R^{1} \times\{0\}\right)} .
\end{aligned}
$$

Thus we find:

$$
\bar{\Gamma} \cap\left(R^{1} \times\{0\}\right)=\overline{\Gamma \cap\left(R^{1} \times\{0\}\right)} .
$$

\subsection{Remark.}

By virtue of the propositions 6.13 and 6.14 , one may conclude: if the problems $(6.3 .1)$ and $(6.10 .10)$ satisfy the conditions of proposition 6.15 , then:

- Problem (6.3.1) possesses an optimal solution.

- The supremum in $(6.3 .1)$ is equal to the infinum in $(6,10.1)$.

We remark that no statement is included with respect to the existence of an optimal solution of problem $(6.10 .1)$.

By virtue of the relations between $1_{1}, 1_{1}^{k}$ and $1_{\infty}, 1_{\infty}^{k}$, as described in $\$ 2,2$, the properties with respect to the problems (6.3.1) and $(6.10 .1)$ axe applicable to our original LP-problem(P- or D-directed; $P$ - and D-regular; $p \pi<1)$ as formulated in $\$ 6.1$. To that end, we transform the LP-problem with the help of a coefficient $\beta \in] 1, \frac{1}{\rho \pi} l$, which is chosen in such a manner that the properties $5.7-a, b$ of proposition 5.6 are valid. Hence, in the next theorem, we shall consider the following LP-problem:

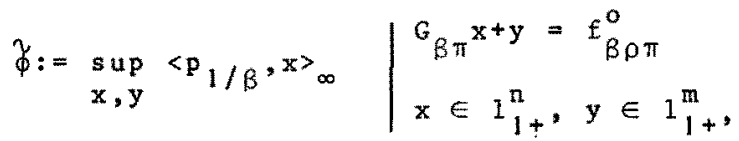

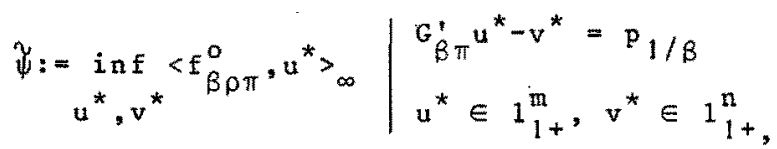


where, $(6.16 .1)$ is the $\beta \pi-t$ ransformed primal problem (6.1.1) and $(6.16 .2)$ the $(1 / \beta \pi)$-transformed dual problem $(6.1 .1)$.

We remark that $\beta \in] 1,1 / 0 \pi\left[, p \in 1_{\infty}^{\mathrm{n}}\right.$, and $\mathrm{f}^{\mathrm{o}} \in 1_{\infty}^{\mathrm{m}}$ imply: $\mathrm{p}_{1 / \beta} \in \mathrm{c}_{0}^{\mathrm{n}}$ and $\mathrm{E}_{B p \pi}^{\mathrm{o}} \in 1_{1}^{\mathrm{m}}$.

The next two properties will show that the matrix $\sigma_{\alpha}$ for every $\alpha>0$ satisfies the conditions $6.3-b$ and $6.15-c$.

\subsection{Proposition.}

Matrices $G_{\alpha}$ and $G_{\alpha}^{\prime}$ (def 6.2.3) generate the following linear operators:

$$
\begin{aligned}
& G_{\alpha}: 1_{\infty}^{n} \rightarrow 1_{\infty}^{m}, G_{\alpha}: 1_{1}^{n} \rightarrow 1_{1}^{m}, G_{\alpha}: c_{0}^{n} \rightarrow c_{0}^{m},(6.17 .1) \\
& G_{\alpha}^{\prime}: 1_{\infty}^{m}+1_{\infty}^{n}, G_{\alpha}^{\prime}: 1_{1}^{m} \rightarrow 1_{1}^{n}, G_{\alpha}^{1}: c_{0}^{m} \rightarrow c_{0}^{n},(6.17 .2)
\end{aligned}
$$

which are all bounded.

\section{Proof.}

Defining

$$
N_{\alpha}:=\sum_{i=1}^{m} \sum_{j=1}^{n}\left(\alpha\left|a_{i j}\right|+\left|b_{i j}\right|\right),
$$

one can derive:

$$
\left\|G_{\alpha} x\right\|_{\infty}^{l, T} \leqq N_{\alpha}\|x\|_{\infty}, \text { for every } T \geqq 1, \quad x \in 1_{\infty}^{n} \text {. }
$$

So, for all $x \in 1_{\infty}^{\text {n }}$ we have:

$$
G_{\alpha} \mathrm{x} \in \mathbb{I}_{\infty}^{\mathrm{m}}, \quad\left\|\mathrm{G}_{\alpha} \mathrm{x}\right\|_{\infty} \leqq \mathrm{N}_{\alpha} \|_{\infty}^{\| \mathrm{x}}{ }_{\infty} .
$$

clearly, matrix $G_{\alpha}$ defines the bounded linear operator $G_{\alpha}: 1_{\infty}^{\mathrm{n}} \rightarrow 1_{\infty}^{\mathrm{m}}$.

In a similar manner, it can be proved that matrix $G_{a}$ defines a bounded linear operator $G_{\alpha}: 1_{1}^{\mathrm{n}} \rightarrow 1_{\infty}^{\mathrm{m}}$. 
Definition $(6.17 .3)$ and definition $(6.2 .1)$ imply:

$$
\left\|G_{\alpha} \times\right\|_{\infty}^{T, T} \leqq N_{\alpha}\|x\|_{\infty}^{T-1, T} \text {, for al1 } T>1, \quad x \in c_{0}^{n} \text {, }
$$

and so for all $x \in c_{0}^{n}$ :

$$
G_{\alpha} x \in \mathrm{c}_{\mathrm{o}}^{\mathrm{m}}
$$

From (6.17.5) and (6.7.4) we may conclude: matrix G generates a bounded linear operator $G_{\alpha}: c_{0}^{n} \rightarrow c_{o}^{m}$.

The statement with respect to the 1 inear operators (6.17.2)

may be proved in a similar manner.

\subsection{Proposition.}

For every $x \in 1_{j}^{n}, u^{*} \in 1_{\infty}^{m}$ :

$$
\left\langle\mathrm{x}, G_{\alpha}^{\prime} \mathrm{u}^{*}\right\rangle_{\infty}=\left\langle\mathrm{u}^{*}, \mathrm{G}_{\alpha} \mathrm{x}\right\rangle_{\infty} .
$$

For every $u \in 1_{1}^{m}, x^{*} \in 1_{\infty}^{n}$ :

$$
\left\langle u, G_{\alpha} x^{*}\right\rangle_{\infty}=\left\langle x^{*}, G_{\alpha}^{*} u\right\rangle_{\infty}
$$

\section{Proof:}

From the definition $(6.2 .1)$ of $G_{\alpha}$ one can derive:

$$
\left.\left\langle x, G_{\alpha}^{\prime} u^{*}\right\rangle_{T}=\left\langle u^{*}, G_{\alpha}\right\rangle_{T}\right\rangle^{+\alpha u^{*}}(T+1){ }^{\prime} A x(T),
$$

for every $x \in 1_{1}^{n}, u^{*} \in I_{\infty}^{m}$, and every $T \geqq 1$.

Since $x \in I_{1}^{n}$ and $u^{*} \in 1_{\infty}^{m}$, we may conclude successively:

$$
\begin{aligned}
& (A x(t)) \rightarrow 0, t \rightarrow \infty, \\
& u^{*}(t+1)^{\prime} \mathrm{Ax}(t) \rightarrow 0, t \rightarrow \infty .
\end{aligned}
$$

The relations $(6.18 .3)$ and $(6.18 .4)$ imply: 


$$
\left\langle\mathrm{x}, \mathrm{G}_{\alpha}^{\prime} \mathrm{u}^{*}\right\rangle_{\infty}=\left\langle\mathrm{u}^{*}, \mathrm{G}_{\alpha} \mathrm{x}\right\rangle_{\infty},
$$

for a $11 \mathrm{x} \in 1_{1}^{\mathfrak{n}}, \mathrm{u}^{*} \in 1_{\infty}^{\mathrm{m}}$.

Equality (6.18.2) may be derived in a similar manner.

\subsection{Theorem.}

For an LP-problem(P- or D-directed; P- and D-regular; $\rho \pi>1)$, the following properties hold:

a) The supremum in the primal problem is equal to the infinum in the dual problem.

b) The primal and dual problems both possess an optimal solution.

c) Numbers $\beta \in 11,1 / 0 \pi\left[, M_{1}>0\right.$, and $M_{2}>0$ exist such that for every P-optimal $x$ and every D-optimal $u$ :

$$
\begin{aligned}
& x_{B \pi} \in 1_{1}^{n}, \quad\left\|x_{B \pi}\right\|_{1} \leqq M_{1} \\
& u_{B p} \in 1_{1}^{m}, \quad\left\|u_{B p}\right\|_{1} \leqq M_{2}
\end{aligned}
$$

d) A feasible solution $((x, y),(u, v))$ is optimal, if and only if simultaneously

$$
\begin{aligned}
& \langle\mathrm{v}, \mathrm{x}\rangle_{\mathrm{T}}+\langle\mathrm{u}, \mathrm{y}\rangle_{\mathrm{T}}=0, \mathrm{~T} \geq 1, \\
& \mathrm{u}(\mathrm{T}+1) \cdot \mathrm{Ax}(\mathrm{T}) \rightarrow 0, \mathrm{~T} \rightarrow \infty,
\end{aligned}
$$

Proof.

Since the $L^{D-p r o b l e m}$ is $p$ - or D-directed and $P$ - and D-regular, and since $\rho \pi<1$, one can derive from proposition 5.6 that numbers $\beta 11,1 /_{\rho \pi}\left[, N_{1}>0\right.$ and $N_{2}>0$ exist for which the following property holds:

Property $1:$ for every $x \in 1_{1+}^{n}, z \in 1_{1+}^{m}$ satisfying 


$$
\left.\begin{array}{l}
\|x\|_{1}>N_{1}+N_{2}\left\|_{1}\right\|_{1} \\
\| f_{B \rho \pi^{2}}^{O}
\end{array}\right\}
$$

a vector $\underline{x} \in 1_{1+}^{n}$ exists such that

$$
\left.\begin{array}{l}
G_{B \pi} \leq f_{B \rho \pi}^{0}+z \\
\left\langle p_{1 / B}, \underline{x}_{\infty}\right\rangle\left\langle p_{1 / B}, x\right\rangle_{\infty} \\
\|\underline{x}\|_{1} \leq N_{1}+N_{2}\|z\|_{1}
\end{array}\right\} .
$$

Since $p_{1 / B} \in c_{o}^{n} \subset 1_{\infty}^{n}$ and $f_{B \rho \pi}^{0} \in 1_{l}^{m}$, we may define set $\Gamma$ in a similar way as done in $(6.3 .3)$, i.e.:

$$
\Gamma:=\left\{\begin{array}{l|l}
(\phi, z) \in\left(R^{1} \times 1_{1}^{m}\right) & \left.\begin{array}{l}
\left.\phi \leqq p_{1 / \beta}, x\right\rangle_{\infty} \\
z=G_{\beta \pi} x+y-f_{B \rho \pi}^{o}, x \in 1_{1+}^{n}, y \in I_{1+}^{m}
\end{array}\right\}
\end{array}\right.
$$

Property 1 and definition (6.19.5) imply:

Property 2: for every $(\phi, z) \in \Gamma \cap\left(R^{1} \times 1_{1+}^{m}\right)$, the system

$$
\left.\begin{array}{l}
G_{\beta \pi} \underline{x} \leqq f_{\beta \rho \pi}^{o}+z \\
\|\underline{x}\|_{1} \leqq N_{1}+N_{2}\left\|_{2}\right\|_{1} \\
\left\langle p_{1 / \beta}, \underline{x}\right\rangle_{\infty} \geqq \phi
\end{array}\right\},
$$

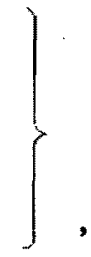

possesses a solution $\underline{x} \in 1_{1+}$.

Since $p_{1 / \beta} \in c_{0}^{n}$ and $f_{\beta p \pi}^{o} \in 1_{1}^{m}$, we may conclude by virtue of the propositions $6.17,6.18$, and of property 2 , that the LP-problems $(6.6 .1)$ and $(6.6 .2)$ satisfy the conditions of proposition 6.13 and 6.15 . Hence, we have: 
Property 3: the supremum $\tilde{\phi}$ in $(6.16 .1)$ is equal to the infinum $\Psi$ in $(6.16 .2)$, and problem $(6.6 .1)$ possesses an optimal solution.

With the help of this result, it will be shown that for the original LP-problem $(6.2 .2)$ :

$$
\begin{aligned}
& \hat{\phi}:=\sup _{x, y}\left\langle p_{\pi}, x\right\rangle_{\infty} \mid G_{1} x+y=f_{\rho}^{0}, x_{\pi} \in 1_{1+}^{n}, y_{\pi} \in 1_{1+}^{m}(6.19 .6) \\
& \hat{\psi}:=\inf \left\langle f_{\rho}^{0}, u\right\rangle_{\infty} \mid G_{1}^{1} u-v=p_{\pi}, u_{\rho} \in 1_{1+}^{m}, v_{\rho} \in 1_{1+}^{n}(6.19 .7)
\end{aligned}
$$

the following properties hold:

4) The supremum $\hat{\phi}$ in $(6.19 .6)$ is equal to the infinum $\hat{\psi}$ in $(6.19 .7)$.

5) Problem (6.19.6) possesses an optimal solution.

6) Numbers $B \in 11,1 / \rho \pi, M_{1}>0$, and $M_{2}>0$ exist, such that for all optimal solutions $x$ of $(6.19 .6)$ and all optimal solutions u of $(6.19 .7)$ :

$$
\begin{aligned}
& x_{B \pi} \in L_{1}^{n}, \quad\left\|x_{B \pi}\right\| \leqq M_{1}, \\
& u_{B \rho} \in 1_{1}^{m}, \quad\left\|u_{B \rho}\right\|_{1} \leqq M_{2} .
\end{aligned}
$$

Clearly, parts $a, b$, and $c$ of the theorem are proved.

(4) Let $(x, y)$ be a feasible solution of $(6.16 .1)$, then $\left(x_{1 / \beta \pi}, y_{1 / \beta \pi}\right)$ is a feasible solution of $(6.19 .6)$. This imp1ies:

$$
\begin{aligned}
\psi & <\hat{\phi}
\end{aligned}
$$

Let $(u, v)$ be a feasible solution of $(6.16 .2)$ then $\left(u_{B \pi}, v_{B \pi}\right)$ is a feasible solution of $(6.19 .7)$. This implies:

$$
\hat{\psi} \leq \psi
$$


Since $\hat{\psi} \geq \phi$ (theorem 4.5) and $\hat{\phi}=\psi$ (property 3 ), the inequalities $(6.19 .8)$ and $(6.19 .9)$ imply $\hat{\phi}=\bar{\phi}=\hat{\psi}=\hat{\psi}$.

(5) Since $\hat{\phi}=\hat{\phi}$, the existence of an optimal solution in (6.16.1) implies the existence of an optimal solution in $(6.19 .6)$.

(6) This property follows from (5) and from the symmetry between the primal and dual.

(7) This property can be derived from theorem 5.7.

To prove part d) of the theorem, we first assume that the LP-problem is D-directed, which implies:

$$
v(t)+A^{\prime} u(t) \geqq 0, \quad t \geq 1
$$

for all D-feasible solutions $(u, v)$ of $(6.2 .2)$.

For all D-feasible solutions $(x, y)$ and $D$-feasible solutions (u,v) (proposition 4.2) the following inequalities hold:

$$
\left.\left\langle\mathrm{P}_{\pi}, \mathrm{x}\right\rangle_{\mathrm{T}}=\left\langle\mathrm{f}_{\mathrm{p}}^{0}, \mathrm{u}\right\rangle_{\mathrm{T}}-\langle\mathrm{u}, \mathrm{y}\rangle_{\mathrm{T}}-\langle\mathrm{v}, \mathrm{x}\rangle_{\mathrm{T}-1}-\left(\mathrm{v}(\mathrm{t})^{\prime}+\mathrm{u}(\mathrm{T}+\mathrm{I})^{\prime} \mathrm{A}\right) \times(\mathrm{T}), \mathrm{T}\right\rangle 1 .
$$

Now, let $(x, y)$ and $(u, v)$ be optimal, then $\left\langle p_{\pi}, x\right\rangle_{\infty}=\left\langle f_{\rho}^{o}, u\right\rangle_{\infty}$, $(6.19 .10),(6.19 .11)$, and the non-negativity of $(x, y),(u, v)$ imply the validity of $(6.19 .3)$ and $(6 \cdot 19.4)$. Hence, these conditions are necessary, in case the D-directedness the LPproblem is assumed.

Now, suppose that the feasible solutions $(\bar{x}, \bar{y})$ and $(\bar{u}, \bar{v})$ satisfy $(6.19 .3)$ and $(6.19 .4)$, then $(6.19 .11)$ implies $\left\langle p_{\pi}, \bar{x}\right\rangle_{\infty}=\left\langle f_{p}^{0}, \bar{u}\right\rangle_{\infty}$. since, for all feasible $(x, y)$ and $(u, v)$ : $\left\langle p_{\pi}, x\right\rangle_{\infty} \leqq \hat{\phi}=\hat{\psi} \leqq\left\langle f_{\rho}^{o}, u\right\rangle_{\infty}$, we may conclude that $(\bar{x}, \bar{y})$ and $(\bar{u}, \vec{v})$ are optimal. This proved the sufficiency of the conditions $(6.19 .3),(6.19 .4)$, in case the D-cirectedness of LP-problem is as sumed. 
When we depart from the assumption that the LP-problem is Pdirected, then inequality:

$$
\operatorname{Ax}(t)+\rho^{t+1} f^{\circ}(t+1) \geq 0, \quad t \geqq 1
$$

may be used. The proof may further be completed in a similar manner.

\subsection{Example.}

A significant difference between the result derived in the last theorem and the we11-known criterium of optimality for linear programing in a finite dimensional space, is constituted by the appearence of the condition

$$
u(T+1)^{\prime} A x(T)+0, T \rightarrow \infty
$$

in theorem 6.19. We shall illustrate the meaning of this condition with the help of the following example, where

$$
\begin{aligned}
& \mathrm{A}:=\left[\begin{array}{rr}
10 & 0 \\
0 & 5
\end{array}\right], \quad \mathrm{B}:=\left[\begin{array}{rr}
9 & 0 \\
0 & 10
\end{array}\right], \quad \mathrm{p}:=\left[\begin{array}{l}
0 \\
1
\end{array}\right], \quad \mathrm{E}:=\left[\begin{array}{l}
0 \\
5
\end{array}\right], \\
& p:=1, \pi:=0,8, \quad x(0):=\left[\begin{array}{l}
1 \\
1
\end{array}\right] \text {. }
\end{aligned}
$$

The corresponding LP-problem is $\mathrm{P}$ - and $\mathrm{D}$-directed, $\mathrm{P}$ - and $\mathrm{D}-$ regular and moreover $p \pi>1$. So, the conditions of theorem 6.19 are satisfied.

For this problem, one may derive that

$$
\begin{aligned}
& \hat{x}(t)=\left[\begin{array}{l}
0 \\
1
\end{array}\right]+\left(\frac{10}{9}\right) t\left[\begin{array}{l}
1 \\
0
\end{array}\right] \\
& \hat{y}(t)=0 \\
& \hat{u}(t)=(0.9)^{t}\left[\begin{array}{c}
0 \\
1 / 6
\end{array}\right] \\
& \hat{v}(t)=\left[\begin{array}{l}
0 \\
0
\end{array}\right] \\
& t \geq 1, \\
& t \geq 1,
\end{aligned}
$$


is an optimal solution, with $\left\langle p_{\pi}, \hat{x}\right\rangle_{\infty}=\left\langle f_{\rho}^{0}, u\right\rangle_{\infty}=4$.

It appears that

$$
\begin{aligned}
& u(t):=(0.8)^{t}\left[\begin{array}{c}
0 \\
1 / 6
\end{array}\right]+(0.9)^{t}\left[\begin{array}{l}
1 \\
0
\end{array}\right] \\
& \dot{v}(t):=\left[\begin{array}{l}
0 \\
0
\end{array}\right]
\end{aligned}
$$

is a D-feasible solution. For this D-feasible solution we find:

$$
\begin{aligned}
& \langle\hat{\mathrm{x}}, \tilde{\mathrm{u}}\rangle_{\mathrm{T}}+\langle\hat{\mathrm{y}}, \tilde{v}\rangle_{\mathrm{T}}=0, \quad \mathrm{~T} \geq 1, \\
& \tilde{u}(T+1) \cdot \hat{A x}(T)=4.5, \quad T \geq 1 \\
& \left\langle f_{\rho}^{\circ}, \tilde{u}\right\rangle_{\infty}=8.5 .
\end{aligned}
$$

Although $(6.19 .2)$ is satisfied, it appears that $(\tilde{u}, \tilde{v})$ is not optimal.

\subsection{Theorem.}

For an LP-problem(P- or D-directed; superregular) the following properties hold:

a) The supremum in the primal problem is equal to the infinum in the dual problem.

b) The primal and dual problems both possess an optimal solution.

c) Positive numbers $M_{1}$ and $M_{2}$ exist such that the following is valid:

A feasible solution $((x, y),(u, v))$ is optimal if and only if simultaneously:

$$
\begin{aligned}
& \left\|\mathrm{x}_{1 / \rho}\right\|_{\infty}^{1, T} \leqq M_{1}, \quad T \geqq 1, \\
& \left\|u_{1 / \pi}\right\|_{\infty}^{1, T} \leq M_{2}, \quad T \geqq 1,
\end{aligned}
$$




$$
\langle v, x\rangle_{T}+\langle u, y\rangle_{T}=C, T \geq 1
$$

\section{Proof.}

The superregularity implies $P$ - and D-regularity and the inequaiity $\rho \pi<1$, and so, by virtue of theorem 6.19 , the validity of a) and b).

Since $\rho \pi<1$, the inequalities $(6.21 .1)$ and $(6.21 .2)$ imply the convergence $\mathrm{u}(\mathrm{T}+1)^{\prime} \mathrm{Ax}(\mathrm{T}) \rightarrow 0, \mathrm{~T}+0$, we may conclude by virtue of theorem 6.19 that $(6.21 .1),(6.21 .2)$, and $(6.21 .3)$ are sufficient conditions for the optimality of a feasible solution $((x, y),(u, v))$.

From theorem 5.8 , the necessity of the conditions $(6.21 .1)$, $(6.21 .2)$, and $(6.21 .3)$, for the optimality of a feasible solution $((x, y),(u, v))$, immediately follows. 


\section{PARAMETRIC PROPERTIES}

\section{I Introduction.}

In this chapter we consider a LP-problem

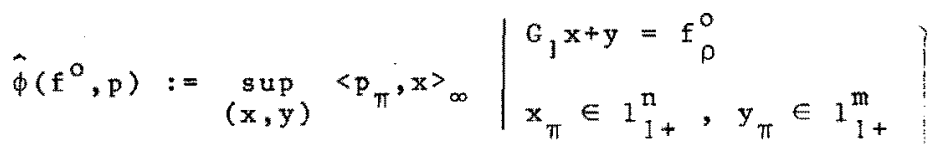

$$
\begin{aligned}
& \psi\left(f^{o}, p\right):=\inf _{(u, v)}\left\langle f_{\rho}^{o}, u\right\rangle_{\infty}\left|\begin{array}{l}
G_{1}^{\prime} u-v=p_{\pi} \\
u_{\rho} \in 1_{1+}^{m}, v_{\rho} \in 1_{1+}^{n}
\end{array}\right|,
\end{aligned}
$$

for all vectors $f^{\circ}, p$, from sets $F^{\circ} \subset 1_{\infty}^{m}, P \subset 1_{\infty}^{n}$ further to be described, with the objective to derive some properties with respect to the continuity of $\hat{\phi}\left(f^{\circ}, p\right)$ and of the set of optimal solutions.

The fixed matrices $A$ and $B$, which generate $G_{1}$, are supposed to be such that a $\mathrm{E}^{\circ}$ or p exists for which the LP-problem is Por $D$-directed. With respect to the fixed quantities $\rho$ and $\pi$ it is supposed that on $<1$.

Further, we restrict ourselves to sets $F^{\circ} \subset 1_{\infty}^{\mathrm{m}}$ and $\mathrm{p} \subset \mathrm{I}_{\infty}^{\mathrm{n}}$ which satisfy the following conditions:

a) $F^{\circ}$ and $P$ are compact.

b) For every $\left(f^{\circ}, p\right) \in F^{\circ} \times p$ the LP-problem $(7.1 .1)$ is superreguiar.

c) For every $\left(f^{\circ}, p\right) \in F^{\circ} \times P$ the LP-problem $(7.1 .1)$ is $P$ - or D-directed.

With respect to the last condition, we remark that the LP-problem is called $P$-directed for every $f^{\circ} \in F^{\circ}$, if for every $f^{\circ} \in F^{\circ}$ a $f \in 1^{u}$ and $a x(0) \in R_{+}^{n}$ exist such that $\left(f(1)+\frac{1}{\rho} A x(0), f(2), \ldots, f(t), \ldots\right)=\left(f^{0}(1), f(2), \ldots, f(t), \ldots\right)$ and such that $f$ satisfies the conditions (2.8) for p-directedness. 
The most important results of this chapter are formulated in the theorems 7.3 and 7.8 .

\subsection{Definitions.}

For the LP-problem and the sets $F^{\circ}$ and $P$ as described above, we define the mappings $X: F^{0} \times P \rightarrow \pi\left(1_{\infty}^{n}\right)(*), X Y: F^{0} \rightarrow \pi\left(1_{\infty}^{n}\right)$, $\hat{X Y}: F^{o} \times P \rightarrow \Pi\left(1_{\infty}^{n}\right) \times \Pi\left(1_{\infty}^{m}\right), \hat{U V}: F^{0} \times P \rightarrow \Pi\left(1_{\infty}^{m}\right) \times \Pi\left(1_{\infty}^{n}\right)$ by:

$$
\begin{aligned}
& X\left(f^{o}, p\right):=\left\{x \in 1_{\infty}^{n} \mid\left\langle p_{\rho \pi}, x\right\rangle_{\infty}=\hat{\phi}\left(f^{o}, p\right)\right\}, \\
& X Y\left(f^{\circ}\right):=\left\{(x, y) \in 1_{\infty+}^{n} \times 1_{\infty+}^{m} \mid G_{1 / 0} x+y=f^{o}\right\}, \\
& \hat{X Y}\left(f^{\circ}, p\right):=X Y\left(f^{\circ}\right) \cap X\left(f^{\circ}, p\right) \times I_{\infty}^{m}, \\
& \hat{\mathrm{UV}}\left(\mathrm{f}^{\circ}, \mathrm{p}\right):=\left\{\begin{array}{l|l}
(\mathrm{u}, \mathrm{v}) \in 1_{\infty+}^{\mathrm{m}} \times 1_{\infty+}^{\mathrm{n}} & \begin{array}{l}
G_{\pi}^{\prime} \mathrm{u}-\mathrm{v}=\mathrm{p} \\
\left\langle\mathrm{f}_{\rho \pi}^{\mathrm{o}}, \mathrm{u}\right\rangle_{\infty}=\dot{\psi}\left(\mathrm{f}^{\circ}, \mathrm{p}\right)
\end{array}
\end{array}\right\} .
\end{aligned}
$$

So for every $f^{\circ} \in F^{\circ}, X Y\left(f^{\circ}\right)$ is the set of $1 / 0$-transforms of $D-$ dominated P-feasible solutions. If $F^{\circ}$ and $P$ satisfy 7.1-a,b,c, then theorem 6.21 and definition (7.2.4) imply that for every $\left(f^{\circ}, p\right) \in F^{\circ} \times P, \hat{X Y}\left(f^{\circ}, p\right)$ is the set of $i / p-t r a n s f o r m s$ of $P-$ optimal solutions of LP-problem (7.1.1). In a similar manner, we may conclude that $\hat{U V}\left(f^{\circ}, p\right)$ is the set of $1 / \pi-t$ ransforms of D-optimal solutions.

Further we wish to introduce two more general concepts.

The graph of a mapping $D: C_{1} \rightarrow \Pi\left(C_{1}\right)$ is the set defined by

$$
\operatorname{graph}\left(C_{1} ; D\right):=\left\{(c, d) \mid c \in C_{1}, d \in D(c)\right\} .
$$

The sphere in $1_{\infty}^{k}$ with a radius $M$ is defined by:

(*) The power set of a set $C$ is denoted by $\Pi(C)$. 


$$
\sigma_{\infty}^{k}(M):=\left\{x \in 1_{\infty}^{k} \mid\|x\|_{\infty} \leqq M\right\}
$$

\subsection{Theorem}

If the sets $F^{\circ} C 1_{\infty}^{m}$ and $P C 1_{\infty}^{n}$ satisfy the conditions $7.1-a, b, c$, then the following properties hold:

a) For every $\left(f^{\circ}, p\right) \in F^{\circ} \times p$ :

- the LP-problem (7.1.1) possesses a p-optimal solution and a D-optimal solution,

- $\hat{X Y}\left(f^{\circ}, \hat{p}\right)$ (def. 7.2 .3$)$ is the set of $1 / p$-transforms of $P$ optimal solutions,

- $\hat{U V}\left(f^{\circ}, p\right)(\operatorname{def}, 7.2 .4)$ is the set of $1 / \pi-t$ ransforms of $n-$ optimal solutions,

$-\hat{\phi}\left(f^{\circ}, p\right)=\hat{\psi}\left(f^{\circ}, p\right)$.

b) Numbers $M_{1}$ and $M_{2}$ exist such that

$$
\begin{aligned}
& \hat{X Y}\left(F^{\circ}, P\right) \subset \sigma_{\infty}^{n}\left(M_{1}\right) \times \sigma_{\infty}^{m}\left(M_{1}\right), \\
& \hat{U V}\left(F^{\circ}, P\right) \subset \sigma_{\infty}^{m}\left(M_{2}\right) \times \sigma_{\infty}^{n}\left(M_{2}\right) .
\end{aligned}
$$

c) Numbers $N_{1}$ and $N_{2}$ exist such that for every $(\tilde{f}, \stackrel{p}{p}),\left(\bar{f}^{o}, \bar{p}\right) \in \bar{F}^{\circ} \times p:$

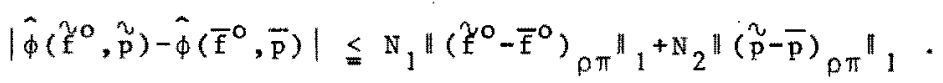

Proof.

(a) For every $\left(f^{\circ}, p\right) \doteq F^{\circ} \times P$ the LP-problem $(7.1 .1)$ satisfies the conditions of theorem 6.19. From this theorem property a) may be derived.

(b) For every $\left(\mathrm{g}^{\circ}, \mathrm{q}\right) \in \mathrm{F}^{\circ} \times \mathrm{p}$, the conditions of theorem 5.8 are satisfied. By virtue of this theorem we may conclude that, for every $\left(g^{\circ}, q\right) \in F^{\circ} \times P$, neighbourhoods $\Omega\left(g^{\circ}\right) \subset 1_{\infty}^{m}$ and $\Omega(q) \subset I_{\infty}^{n}$ and a number $M\left(g^{\circ}, q\right)$ exist such that

$$
\hat{X Y}\left(\Omega\left(g^{o}\right) \cap F^{\circ}, \Omega(q) \cap P\right) \subset \sigma_{\infty}^{n}\left(M\left(g^{\circ}, q\right)\right) \times 1^{m} .
$$


Since $G_{1 / \rho}: 1_{\infty}^{\mathfrak{n}} \rightarrow 1_{\infty}^{\mathrm{m}}$ is a bounded linear operator, this implies the existence of a number $M_{I}\left(g^{\circ}, q\right)$ such that

$$
\hat{X Y}\left(\Omega\left(g^{o}\right) \cap F^{0}, \Omega(q) \cap P\right) \subset \sigma_{\infty}^{n}\left(M_{1}\left(g^{0}, q\right)\right) \times \sigma_{\infty}^{m}\left(M_{1}\left(g^{0}, q\right)\right) .
$$

Since the sets $F^{\circ}$ and $P$ are supposed to be compact, a finite number of vectors $\left(g_{1}^{0}, q_{1}\right) \in F^{0} \times P, I=1,2, \ldots, L$ exist (Heine-Bore1) such that

$$
\bigcup_{l=1}^{L} \lambda\left(g_{1}^{0}\right) \times \Omega\left(q_{1}\right) \supset F^{0} \times P .
$$

The ralations (7.3.1) and (7.3.2) imply the existence of a number $M_{1}$ such that

$$
\hat{X Y}\left(F^{\circ} \times P\right) \subset \sigma_{\infty}^{n}\left(M_{1}\right) \times \sigma_{\infty}^{m}\left(M_{1}\right) .
$$

Property b) for set $\hat{U V}\left(F^{\circ} \times P\right)$ then follows from the symmetry between primal and dual system.

(c) Since, for every $\left(f^{\circ}, p\right) \in F^{\circ} \times P, \hat{U V}\left(f^{\circ}, p\right)$ is the set of $\frac{1}{\pi}$-transforms of D-optimal solutions, we have, for every $\left(\tilde{\mathrm{F}}^{\circ}, \tilde{\mathrm{P}}, \tilde{\mathrm{u}}, \tilde{v}\right),\left(\overline{\mathrm{f}}^{\circ}, \overline{\mathrm{p}}, \overline{\mathrm{u}}, \bar{v}\right) \in \operatorname{graph}\left(\mathrm{F}^{\circ} \times \mathrm{P} ; \hat{\mathrm{U}} \hat{\mathrm{V}}\right):$

$$
\begin{aligned}
& \hat{\phi}\left(\tilde{f}^{\circ}, p\right)=\left\langle\tilde{f}_{\rho \pi}^{0}, \tilde{u}\right\rangle_{\infty}, \\
& \hat{\phi}\left(\overline{\mathrm{f}}^{\circ}, \mathrm{p}\right)=\left\langle\overline{\mathrm{f}}_{\rho \pi}^{\circ}, \overline{\mathrm{u}}\right\rangle_{\infty}, \\
& \bar{\phi}\left(\mathfrak{f}^{\circ}, p\right) \leqq\left\langle\hat{f}_{\rho \pi}, \bar{u}\right\rangle_{\infty}, \\
& \hat{\phi}\left(\bar{f}^{\circ}, p\right) \leqq\left\langle\bar{f} \bar{\rho}_{\rho \pi}, \tilde{u}_{\infty}\right.
\end{aligned}
$$

The relations $(7.3 .3)$ and $(7.3 .6)$ imply

$$
\hat{\phi}\left(f^{0}, p\right)-\hat{\phi}\left(\tilde{f}^{0}, p\right) \leqq\left\langle f_{\rho \pi}^{0}-f_{\rho \pi}^{0}, \tilde{u}\right\rangle_{\infty} .
$$


$(7.3 .4)$ and $(7.3 \cdot 5)$ imp $1 y$

$$
-\hat{\phi}\left(\overline{\mathrm{f}}^{\circ}, p\right)+\hat{\phi}\left(\tilde{f}^{\circ}, p\right) \geqq\left\langle\tilde{f}_{\rho \pi}^{\circ}-\overline{\mathrm{f}}_{\rho \pi}^{\circ}, \overline{\mathrm{u}}\right\rangle_{\infty}
$$

From 7.3-b it follows:

$$
\begin{aligned}
& \left|\left\langle\overline{\mathrm{f}}_{\rho \pi}^{\circ}-\mathfrak{f}_{\rho \pi}^{\circ}, \tilde{u}\right\rangle_{\infty}\right| \leqq M_{2} \|\left(\tilde{f}^{\circ}-\bar{f}^{\circ}\right) \rho \pi^{\|} 1 \\
& \left|\left\langle\gtrless_{\rho \pi}^{\circ}-\bar{f}_{\rho \pi}, \bar{u}\right\rangle_{\infty}\right| \leqq M_{2} \|\left(\mathfrak{f}^{\circ}-\bar{f}^{\circ}\right) \rho \pi^{\|} 1
\end{aligned}
$$

The inequalities $(7.3 .7),(7.3 .8)$ and $(7.3 .9)$ imply

$$
\left|\hat{\phi}\left(\mathfrak{f}^{\circ}, p\right)-\hat{\phi}\left(\bar{f}^{\circ}, p\right)\right| \leqq M_{2}\left\|\left(\mathfrak{f}^{\circ}-\bar{f}^{o}\right) \rho\right\|_{1}
$$

In connection with the symmetry between the primal and dual problem we may also conclude that a number $N$ exists such that, for every $\left(f^{\circ}, \tilde{p}\right),\left(f^{\circ}, \bar{p}\right) \in F^{\circ} \times P:$

$$
\left|\hat{\phi}\left(f^{0}, \tilde{p}\right)-\hat{\phi}\left(f^{0}, \bar{p}\right)\right| \leqq N\left\|(\tilde{p}-\bar{p}){ }_{\rho \pi}\right\|
$$

From (7.3.10) and (7.3.11) one may easily derive that property c) holds.

\subsection{Proposition.}

If the sets $F^{\circ} \subset 1_{\infty}^{m}$ and $P \subset 1_{\infty}^{n}$ satisfy the conditions 7.1-a,b,c, then the graph of the mapping $X: F^{\circ} \times p+\Pi\left(I_{\infty}^{n}\right)$ def 7.2 .1$)$ is weak* closed.

\section{Proof.}

Suppose $\left\{\left(f^{i}, p^{i}, x^{i}\right)\right\}_{1}^{\infty}$ is a sequence in the graph $\left(F^{\circ} \times P ; X\right)$ which converges weak to a point $\left(f^{\circ}, p^{\circ}, x^{\circ}\right) ;$ i.e.

$$
\begin{aligned}
& \left\{\left(f^{i}, p^{i}, x^{i}\right)\right\}_{1}^{\infty} \subset \operatorname{graph}\left(F^{o} \times P ; x\right), \\
& \left(f^{i}, p^{i}, x^{i}\right) \stackrel{\sim}{\rightarrow}\left(f^{o}, p^{o}, x^{o}\right), \quad i \rightarrow \infty .
\end{aligned}
$$


For such a sequence we shall show successively:

1) $\left(E^{\circ}, p^{o}\right) \in E^{\circ} \times P$.

2) $\hat{\phi}\left(f^{i}, p^{i}\right)+\hat{\phi}\left(f^{0}, p^{0}\right), t+\infty$.

3) $\left\langle p_{p \pi}^{i}, x^{i}\right\rangle_{\infty} \rightarrow\left\langle p_{\rho \pi}^{0}, x^{0}\right\rangle_{\infty}, \quad i \rightarrow \infty$.

4) $\left\langle p_{\rho \pi}^{0}, x^{0}\right\rangle_{\infty}=\hat{\phi}\left(E^{0}, p^{0}\right)$,

5) $\left(\mathrm{f}^{\circ}, \mathrm{p}^{\circ}, \mathrm{x}^{0}\right) \in \mathrm{graph}\left(\mathrm{F}^{\circ} \times \mathrm{P} ; \mathrm{X}\right)$.

Then, from 5), (7.5.1) and from (7.5.2), we may conclude that graph $\left(F^{\circ} \times P ; X\right)$ is weak* closed.

(1) This property immediately follows from (7.4.1), (7.4.2) and from the supposition that $F^{\circ}$ and $P$ are compact and so weak* compact as well.

(2) Since $\left[\left(f^{i}, p^{i}\right)\right\}_{0}^{\infty} \subset F^{\circ} \times P$ and since the sets $F^{\circ} \times P$ isatisfy the conditions 7.1-a,b,c, it follows from theorem 7.3-c that numbers $N_{1}$ and $N_{2}$ exist such that:

$$
\begin{aligned}
& \left|\hat{\phi}\left(f^{i}, p^{i}\right)-\hat{\phi}\left(f^{\circ}, p^{0}\right)\right| \leqq N_{1}\left\|\left(f^{i}-f^{0}\right) \rho \pi_{1}+M_{2}\right\|\left(p^{i}-p^{0}\right) p \|_{1}, \\
& i \geq 0
\end{aligned}
$$

Since the sets $F^{\circ}$ and $P$ are bounded, a number $N_{3}$ exists such that

$$
\left\{\left(f^{i}, p^{i}\right)\right\}_{0}^{\infty} \subset \sigma_{\infty}^{m}\left(N_{3}\right) \times \sigma_{\infty}^{n}\left(N_{3}\right) .
$$

The relations $(7.4 .3)$ and $(7.4 .4)$ imply:

$$
\begin{aligned}
\left|\hat{\phi}\left(f^{i}, p^{i}\right)-\hat{\phi}\left(f^{o}, p^{o}\right)\right| & \leqq N_{1}\left\|\left(f^{i}-f^{o}\right) \rho \pi^{1}, T_{+N}\right\|\left(p^{i}-p^{o}\right) \rho \|^{1}, T+ \\
& +(\rho \pi)^{T}(m+n) N_{3}, i \geq 0, T \geqq 1 \cdot(7.4 .5)
\end{aligned}
$$


From (7.4.2), (7.4.5) and from the supposition that $\rho \pi>1$, one may deduct that

$$
\hat{\phi}\left(f^{i}, p^{i}\right) \rightarrow \hat{\phi}\left(f^{o}, p^{o}\right), \quad i \rightarrow \infty .
$$

(3) Since $(7.4 .2)$ implies $(1: 270)$ that $\left\{p^{i}\right\}_{1}^{\infty}$ and $\left\{x^{i}\right\}_{1}^{\infty}$ are located in bounded sets of $1_{\infty}^{n}$, and since $\left.\gamma:=\sqrt{\rho \pi} \in\right] 0,1[$, one can derive from (7.4.2):

$$
\left.\begin{array}{l}
p_{\gamma}^{i}+p_{\gamma}^{0}, i+\infty \\
x_{\gamma}^{i}+x_{\gamma}^{0}, i \rightarrow \infty
\end{array}\right\} .
$$

since $\left\{p_{\gamma}^{i}\right\}_{0}^{\infty}$ and $\left\{x_{\gamma}^{i}\right\}_{0}^{\infty}$ are located in bounded sets of $1_{1}^{n}$, and since

$\left|\left\langle p_{\rho \pi}^{i}, x^{i}\right\rangle_{\infty}-\left\langle p_{\rho \pi}^{o}, x^{o}\right\rangle_{\infty}\right|=\left|\left\langle p_{\gamma}^{i}, x_{\gamma}^{i}-x_{\gamma}^{o}\right\rangle_{\infty}-\left\langle p_{\gamma}^{o}-p_{\gamma}^{i}, x_{\gamma}^{o}\right\rangle_{\infty}\right|, i \geqq 0$, one can derive that (7.4.6) implies property 3$)$.

(4) Since $\left\langle p_{\rho \pi}^{i}, x^{i}\right\rangle_{\infty}=\hat{\phi}\left(f^{i}, p^{i}\right), i \geqq 1$, this property immediately follows from 2) and 3 ).

(5) This property follows immediately from 1), 4) and from the definition of the mapping $x$.

\subsection{Proposition.}

If the set $F^{\circ} \subset 1_{\infty}^{m}$ satisfies the conditions $7.1-a, b, c$, then the graph of the mapping XY $: F^{o} \rightarrow \Pi\left(1_{\infty}^{n}\right) \times I I\left(l_{\infty}^{m}\right)$ (def 7.2.2) is weak* closed.

\section{Proof.}

Suppose $\left\{\left(f^{i}, x^{i}, y^{i}\right)\right\}_{1}^{\infty}$ is a sequence in the graph of XY which converges weak ${ }^{*}$ to a point $\left(f^{\circ}, x^{\circ}, y^{\circ}\right) ;$ i.e.:

$$
\begin{aligned}
& \left\{\left(f^{i}, x^{i}, y^{i}\right)\right\}_{1}^{\infty} \subset \operatorname{graph}\left(F^{\circ} ; X Y\right), \\
& \left(f^{i}, x^{i}, y^{i}\right) \rightarrow\left(f^{\circ}, x^{0}, y^{\circ}\right), i \rightarrow \infty .
\end{aligned}
$$


For such a sequence we shall show that:

1) $\AA^{\circ} \in \mathbb{F}^{\circ}$.

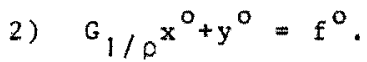

3) $\left(f^{\circ}, x^{\circ}, y^{\circ}\right) \in \operatorname{graph}\left(F^{\circ} ; X Y\right)$ :

Then, from c), (7.5.1) and from (7.5.2), we may conclude that graph $\left(\mathrm{F}^{\circ} ; \mathrm{XY}\right)$ is weak* closed.

(1) This property immediately follows from (7.5.1), (7.5.2) and from the supposition that $\bar{F}^{\circ}$ is compact and so weak* compact as well.

(2) Since $\left\{\left(f^{i}, x^{i}, y^{i}\right)\right\}_{1}^{\infty} \subset$ graph $\left(F^{\circ} ; X Y\right)$, we have:

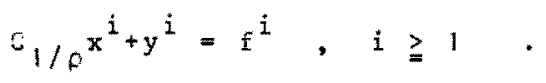

Sinse $G_{1 / 0}^{\prime}: 1_{1}^{m}+1_{1}^{n}$ is a bounded linear operator (Prop. 6.17) and in addition (prop. 6.18), for every $w \in 1_{1}^{m}, x \in 1_{\infty}^{n}$ :

$$
\left\langle w, G_{1} / \rho^{x}\right\rangle_{\infty}=\left\langle G i j \rho^{w}, x\right\rangle_{\infty}
$$

The relations $(7.5 .2)$ and $(7.5 .3)$ imply, for every $w \in 1_{1}^{\mathrm{m}}$ :

$$
\left\langle w, f^{i}\right\rangle_{\infty} \rightarrow\left\langle w, G_{1} / 0^{\left.x^{0}+y^{0}\right\rangle_{\infty}, \quad i \rightarrow \infty .}\right.
$$

Clearly,

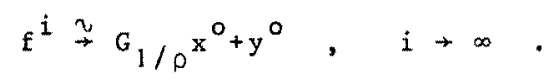

From (7.5.2) and from (7.5.4) we may conclude

$$
G_{1 / 0^{x}+y^{0}}=E^{0} .
$$

(3) The definition $(7,2,2)$ of the mapping $X Y$ implies

$$
\left\{\left(x^{i}, y^{i}\right)\right\}_{1}^{\infty} \subset 1_{\infty+}^{n} \times 1_{\infty+}^{m} .
$$


Since $1_{\infty+}^{\mathrm{n}}$ and $1_{\infty}^{\mathrm{m}}$ are weak ${ }^{*}$ closed and $\left(x^{i}, y^{i}\right) \stackrel{\sim}{\rightarrow}\left(x^{0}, y^{0}\right)$, $i \rightarrow \infty$, we have:

$$
\left(x^{0}, y^{0}\right) \in 1_{\infty}^{n} \times 1_{\infty+}^{m} .
$$

On the ground that $(7.5 .5)$ and $(7.5 .6)$ we may conclude that $\left(f^{\circ}, x^{\circ}, y^{\circ}\right) \in \operatorname{graph}\left(F^{\circ} ; X Y\right)$.

\subsection{Proposition.}

If the sets $F^{\circ} \subset 1_{\infty}^{\mathrm{m}}$ and $P \subset 1_{\infty}^{\mathrm{n}}$ satisfy the conditions $7.4-a, b, c$, then the graph of the mapping $\hat{X Y}: F^{0} \times P \rightarrow \Pi\left(1_{\infty}^{n}\right) \times \Pi\left(1_{\infty}^{m}\right)($ def 7.2 .3$)$ is weak* compact.

\section{Proof.}

From theorem $7.3-b$ and from (7.2.3) we may conclude that a number M exists such that

$$
\begin{aligned}
\operatorname{graph} & \left(F^{\circ} \times P, \hat{X Y}\right)=\left(\operatorname{graph}\left(F^{\circ} \times P ; X\right) \times 1_{\infty}^{m}\right) \cap \\
& \cap\left\{\left(f^{\circ}, p, x, y\right) \mid\left(f^{\circ}, x, y\right) \in \operatorname{graph}\left(F^{\circ}, X Y\right), p \in P\right\} \cap \\
& \cap\left(F^{\circ} \times P \times \sigma_{\infty}^{n}(M) \times \sigma_{\infty}^{m}(M)\right)
\end{aligned}
$$

Since $F^{\circ}$ and $P$ are weak ${ }^{*}$ compact, the sets of the right-hand side of (7.6.1) possess the following properties:

- the first set is weak ${ }^{*}$ closed (prop. 7.4),

- the second set is weak* closed (prop. 7.5),

- the third set is weak* compact (Alaoglu's theorem 1:272).

Hence, we may conclude that $\mathrm{graph}\left(\mathrm{F}^{\mathrm{o}} \times \mathrm{P} ; \hat{\mathrm{XY}}\right)$ is weak ${ }^{*}$ compact.

\subsection{Proposition.}

If the sets $F^{\circ} \subset 1_{\infty}^{\mathrm{m}}$ and $P \subset 1_{\infty}^{\mathrm{n}}$ satisfy the conditions $7.2-a, b, c$, then the mapping $\hat{X Y}: F^{0} \times P \rightarrow \Pi\left(1_{\infty}^{n}\right) \times \Pi\left(I_{\infty}^{m}\right)$

(def 7.2.3) possesses the following property: 
For every $\left(f^{\circ}, p\right) \in F^{0} \times P$ and every weak ${ }^{*}$ open set $Q \subset 1_{\infty}^{n} \times 1{ }_{\infty}^{m}$. such that $Q \supset \hat{X Y}\left(f^{\circ}, p\right)$ a neighbourhood $\delta\left(f^{\circ}, p\right) \subset 1_{\infty}^{m_{\infty}} 1_{\infty}^{n^{\infty}}$ exists for which

$$
\hat{X Y Y}\left(\Omega\left(E^{\circ}, P\right) \cap F^{\circ} \times P\right) \subset Q
$$

Proof.

Suppose that for some $\left(f^{\circ}, p\right) \in F^{\circ} \times P$ and some weak open set $Q \subset 1_{\infty}^{n} \times 1_{\infty}^{n}$ such that $Q>\hat{X} \hat{Y}\left(f^{\circ}, p\right)$, no neighbourhood $\Omega\left(f^{0}, p\right) \subset F^{0} \times P$ exists for which $(7.7 .1)$ holds.

Denoting the complement of $Q$ with respect to $1_{\infty}^{n} \times 1_{\infty}^{m}$ as $Q^{c}$, this supposition implies the existence of a sequence $\left\{\left(E^{i}, p^{i}, x^{i}, y^{i}\right)\right\}_{1}^{\infty}$ such that

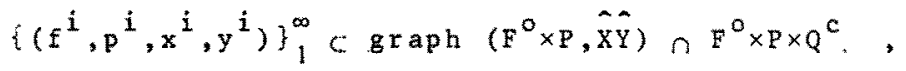

$$
\begin{aligned}
& \left(E^{i}, p^{i}\right) \rightarrow\left(f^{0}, p\right), \quad i \rightarrow \infty .
\end{aligned}
$$

Since $Q^{C}$ is weak* closed, $F^{\circ} \times P$ is compact and since graph $\left(F^{\circ} \times p, \hat{X Y}\right)$ is weak* compact (prop. 7.6), the right hand term of $(7.7 .2)$ is weak* compact.

This implies that subsequence $\left\{\left(f^{i(k)}, p^{i(k)}, x^{i(k)}, y^{i(k)}\right)\right\}_{k=1}^{\infty}$ and a point $(\overline{\mathrm{f}}, \overline{\mathrm{p}}, \overline{\mathrm{x}}, \overline{\mathrm{y}})$ exist such that

$$
\begin{aligned}
& \left.\left(\mathrm{f}^{i(k)}, \mathrm{p}^{i(k)}, \mathrm{x}^{i(k)}, \mathrm{y}^{i(k)}\right) \stackrel{\because}{\rightarrow} \overline{\mathrm{f}}, \overline{\mathrm{p}}, \overline{\mathrm{x}}, \overline{\mathrm{y}}\right), \mathrm{k} \rightarrow \infty,(7.7 .4) \\
& (\overline{\mathrm{f}}, \overline{\mathrm{p}}, \overline{\mathrm{x}}, \overline{\mathrm{y}}) \in \mathrm{graph}\left(\mathrm{F}^{0} \times \mathrm{p} ; \hat{\mathrm{XY}}\right) \cap \mathrm{F}^{0} \times \mathrm{P} \times \mathrm{Q}^{\mathrm{c}} .(7.7 .5)
\end{aligned}
$$

The convergence $\left(E^{i}, p^{i}\right) \rightarrow\left(f^{\circ}, p\right)$ and $(7.7 .4)$ imply $(\bar{f}, \bar{p})=$ $\left(f^{\circ}, p\right)$ and so, on account of $(7.7 .5)$,

$$
\left(f^{\circ}, p, \bar{x}, \bar{y}\right) \in \operatorname{graph}\left(F^{\circ} \times P ; \hat{X Y}\right)
$$

as we11.

Since $Q \supset \widehat{X Y}\left(f^{\circ}, p\right)$, it then follows that 


$$
(\bar{x}, \bar{y}) \in Q
$$

However, $(7.7 .5)$ implies $(\bar{x}, \bar{y}) \notin Q ;$ so the supposition leads to a contradiction.

\subsection{Theorem.}

If $F^{0} C 1_{\infty}^{m}$ and $P \subset 1_{\infty}^{n}$ satisfy the conditions $7.2-a, b, c$, then the mappings $\hat{X Y}: F^{\circ} \times P \rightarrow \Pi\left(1_{\infty}^{n}\right) \times \Pi\left(1_{\infty}^{\mathrm{m}}\right)$ (def 7.2 .3$)$ and

UV : $F^{o} \times p+\Pi\left(1_{\infty}^{m}\right) \times \pi\left(1_{\infty}^{n}\right)$ possess the following property:

For every $\left(f^{0}, p\right) \in F^{0} \times p$ and every open set $Q_{1} \subset 1_{\infty}^{n} \times 1_{\infty}^{m}$, $Q_{2} \subset 1_{\infty}^{m} \times 1_{\infty}^{n}$ such that $Q_{1} \supset \hat{X Y}\left(f^{\circ}, p\right), Q_{2} \supset \hat{U V}\left(f^{\circ}, p\right)$ a neighbourhood $\Omega\left(f^{\circ}, p\right) \subset 1_{\infty}^{m_{\infty}} \times 1_{\infty}^{n}$ exists for which:

$$
\begin{aligned}
& \hat{X Y}\left(\Omega\left(F^{\circ}, p\right) \cap F^{\circ} \times P\right) \subset Q_{1}, \\
& \hat{U V}\left(\Omega\left(F^{\circ}, p\right) \cap F^{\circ} \times P\right) \subset Q_{2} .
\end{aligned}
$$

\section{Proof:}

Suppose that $\left(\mathrm{F}^{\mathrm{o}}, \mathrm{p}\right) \in \mathrm{F}^{0} \times \mathrm{P}$ and the open set $Q_{1} \subset 1_{\infty}^{\mathrm{n}} \times 1_{\infty}^{\mathrm{m}}$, $Q_{2} \subset 1_{\infty}^{m} \times 1_{\infty}^{\mathrm{n}}$ are such that

$$
\begin{aligned}
& Q_{1} \supset \hat{X Y}\left(f^{\circ}, p\right) \\
& q_{2} \supset \hat{U V}\left(f^{\circ}, p\right)
\end{aligned}
$$

Since every open set in $1_{\infty}^{n} \times l_{\infty}^{m}$ is also weak* open, we may conclude, by virtue of proposition 7.7 , that a neighbourhood $\Omega_{1}\left(f^{\circ} p\right) \subset I_{\infty}^{m} \times 1_{\infty}^{n}$ exists, such that

$$
\hat{X} \hat{Y}\left(\Omega_{1}\left(f^{\circ}, P\right) \cap E^{o} \times P\right) \subset Q_{1} .
$$

Then, from the symmetry between the primal and dual systems, we also may conclude that a neighbourhood $\Omega_{2}\left(f^{0}, p\right) \subset 1_{\infty}^{n} \times 1_{\infty}^{m}$ exists, satisfying 


$$
\hat{U V}\left(\Omega_{2}\left(f^{\circ}, p\right) \cap F^{\circ} \times P\right) \subset Q_{2} .
$$

Putting $\Omega\left(\mathrm{f}^{\circ}, \mathrm{p}\right):=\Omega_{1}\left(\mathrm{f}^{\circ}, \mathrm{p}\right) \cap \Omega_{2}\left(\mathrm{f}^{\circ}, \mathrm{p}\right)$, it follows from $(7.8 .5)$ and $(7.8 .6)$ that

$$
\begin{aligned}
& \hat{X Y}\left(\Omega\left(f^{\circ}, p\right) \cap F^{0} \times p\right) \subset Q_{1}, \\
& \hat{U V}\left(\Omega\left(f^{0}, p\right) \cap F^{0} \times P\right) \subset Q_{2},
\end{aligned}
$$

\subsection{Remark.}

Theorem 7.8 is an analogy of the well known property of upper semicontinuity (1:115) of the set of optimal solutions of a LP-proulem in a finite dimensional space. In order to formulate this property, consider the following LP-problem in a finite dimensional euclidean space:

$$
\begin{aligned}
& \hat{\phi}(g, q):=\max _{(x, y)} q^{\prime} x \quad \mid \begin{aligned}
c x+y & =g \\
x, y & \geqq 0,
\end{aligned} \\
& \hat{\psi}(g, q):=\min _{(u, v)} g^{\prime} u \quad\left\{\begin{array}{r}
c^{\prime} u-v=q \\
u, v \geq 0,
\end{array}\right.
\end{aligned}
$$

where, $q \in E^{n}, g \in E^{m}$ and $c$ is a $n \times n-m a t r i x$.

Defining $\overline{X Y}: R^{m+n} \rightarrow \pi\left(R^{n+m}\right)$ and $\overline{U V}: R^{m+n}+\pi\left(R^{m+n}\right)$ by

$$
\begin{aligned}
& \left.\overline{X Y}(g, q):=(x, y) \in R^{n+m} \mid \begin{array}{l}
c x+y=g \\
q^{\prime} x=\hat{\phi}(g, q)
\end{array}\right\}
\end{aligned}
$$

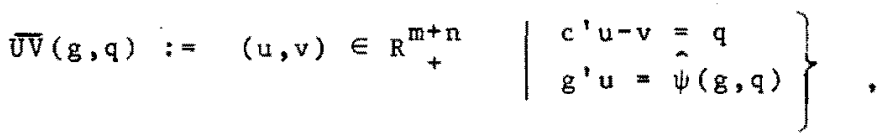

the following property holds:

If the systems 


$$
\begin{aligned}
& \left.\begin{array}{rl}
c x+y & =\bar{g} \\
x, y & >0
\end{array}\right\}, \\
& \left.\begin{array}{rl}
c^{\prime} u-v & =\bar{q} \\
u, v & >0
\end{array}\right\},
\end{aligned}
$$

are solvable, then for every open $Q_{1} \supset \overline{X Y}(\bar{g}, \bar{q})$ and every open $Q_{2} \supset \overline{U V}(\bar{g}, \bar{q})$, a neigbourhood $\Omega(\bar{g}, \bar{q}) \subset R^{m+n}$ exists such that

$$
\begin{aligned}
& \overline{X Y}\left(\Omega(\bar{g}, \bar{q}) \subset Q_{1}\right. \\
& \overline{\mathrm{UV}}\left(\Omega(\overline{\mathrm{g}}, \overline{\mathrm{q}}) \subset \mathrm{Q}_{2}\right\} \text {. }
\end{aligned}
$$

In that case, we say that $X Y$ and UV are upper semicontinuous at $(\bar{g}, \bar{q})$. 
8. PATHS OF EQUILIBRIUM.

\section{I Introduction.}

In this chapter we considex a very special type of feasible solutions of the LF-problem(P- or D-directed; $P^{\circ}$ - and Dfeasible; $f(t)=q, p(t)=\tilde{p}, t \geqq 1 ; \rho \pi<1)$. To that end, we investigate the following system:

$$
\left.\begin{array}{l}
\left(B-\frac{1}{\rho} A\right) \tilde{x}+\tilde{y}=\tilde{F} \\
\left(B^{\prime}-\pi A^{\prime}\right) \tilde{u}-\tilde{v}=\tilde{p} \\
\tilde{v} \cdot \tilde{x}+\tilde{u} \cdot \tilde{y}=0
\end{array}\right\} .
$$

If $(\tilde{x}, \tilde{y}) \in R^{n+m},(\tilde{u}, \tilde{v}) \in R^{n+m}$ satisfies $(8.1 .1)$, then it may be verified that, $(x, y)$ defined by

$$
(x(t), y(t)):=p^{t}(\hat{x}, \hat{y}), \quad t \geqq 1,
$$

is a P-feasible solution for the LP-system with an initial vector $x(0)=\tilde{x}$, and that $(u, v)$, defined by

$$
(u(t), v(t)):=\pi^{t}(\tilde{u}, \tilde{v}), \quad t \geqq 1,
$$

is a D-feasible solution of this LP-system.

Moreover, $((x, y),(u, v))$ satisfies:

$$
\begin{array}{ll}
\langle v, x\rangle_{T}+\langle u, y\rangle_{T}=0, & T \geqq 0 \\
u(T+1){ }^{\prime} A x(T) \rightarrow 0, & T \rightarrow 0, \\
\left\langle p_{\pi}, x\right\rangle_{T} \rightarrow\left\langle p_{\pi}, x\right\rangle_{\infty}, & T \rightarrow \infty, \\
\left\langle f_{\rho}^{0}, u\right\rangle_{T} \rightarrow\left\langle f^{0}, u\right\rangle_{\infty}, & T \rightarrow \infty, \\
\left\langle p_{\pi}, x\right\rangle_{\infty}=\left\langle E_{\rho}^{0}, u\right\rangle_{\infty}, & \text { (proposition 4.2) }
\end{array}
$$


So, by virtue of theorem 4.5, we may conclude: $(x, y)$ is $p$ optimal and $(u, v)$ is D-optimal.

A combination of non-negative vectors $(\hat{x}, \hat{y}),(\hat{u}, \tilde{v})$ satisfying $(8.1 .1)$ we shall call an equilibrium combination ${ }^{(*)}$; the combination of vectors $((x, y),(u, v))$, defined by $(8.1 .2)$ and (8.1.3), is called a path of equilibrium. Clearly, these concepts are only sensible for an LP-problem which is exponential (i.e.: $f(t)=\tilde{f}, p(t)=\tilde{p}, t \geqq 1)$.

First we shall prove that every LP-problem(P- or D-directed; exponential; intial strong regular) possesses an equilibrium combination.

\subsection{Definitions.}

We consider, for all $q \in \mathrm{R}^{\mathrm{n}}$, the following linear programming problem in a finite dimensional Euclidean space:

$$
\begin{aligned}
& \phi(q):=\max _{x, y}(p-q) \cdot x \mid\left(B-\frac{1}{\rho} A\right) x+y=\tilde{f}, x \in \mathbb{R}_{+}^{n}, y \in \mathbb{R}_{+}^{m}, \\
& \psi(q):=\min _{u, v} f^{\prime} u \mid\left(B^{\prime}-\frac{1}{\rho} A^{\prime}\right) u-v=\tilde{p}-q, u \in R_{+}^{m}, v \in R_{+}^{n} .
\end{aligned}
$$

We shall memorate some properties of these problems:

a) Problem (8.2.1) or (8.2.2) possesses an optimal solution, if and only if the problems are both feasible. In that case: $\phi(q)=\psi(q)$.

b) Feasible solutions $(\bar{x}, \bar{y})$ and $(\bar{u}, \bar{v})$ of $(8.2 .1),(8.2 .2)$ resp. are both optimal, if and only if

(*) In some degree, this problematic is related to that treated by von Neumann: "A model of general economic equilibrium".(11) 


$$
\bar{v}^{\prime} x+\bar{u}^{\prime} y=0
$$

c) If $(8,2,1)$ possesses a feasible solution $x, y>0$, and $Q \subset E^{n}$ is a compact set such that, for every $q \in Q$, problem (8.2.2) has a feasible solution $u, v>0$, then the mappings $\underline{X}: \mathrm{R}^{\mathrm{n}} \rightarrow \pi\left(\mathrm{R}^{\mathrm{n}}\right), \underline{\mathrm{U}}: \mathrm{R}^{\mathrm{n}} \rightarrow \pi\left(\mathrm{R}^{\mathrm{m}}\right)$ defined by:

$$
\begin{aligned}
& X(q):=\left\{x \in R_{+}^{n} \mid \begin{array}{l}
(\hat{p}-q){ }^{\prime} x=\phi(q) \\
\left(B-\frac{1}{p} A\right) x \leqq z
\end{array}\right\} \\
& \underline{U}(q):=\because u \in \mathbb{R}_{+}^{\mathbb{M}} \cdot \tilde{f} u=\psi(q) \\
& \left(B^{\prime}-\frac{1}{\rho} A^{\prime}\right) u \geqq p-q
\end{aligned}
$$

are upper semicontinuous on $Q$ (see $\overline{8} .9$ ). Note: $X(q)$ and U(q) are the sets of optimal solutions of $(8.2 .1),(8.2 .2)$ resp.

Further, we introduce the matrices $C(n \times m)$ and $D$ (diagonal $m \times m)$ :

$$
\begin{array}{ll}
c_{i .}=\left(\frac{1}{\rho \pi}-1\right) b_{i .} & \text {, if } b_{i .} \geq 0 \\
c_{i .}=\left(\frac{1}{\rho}-\pi\right) a_{i .} & \text {, if } b_{i .} \geq 0 \\
d_{i i}=\frac{1}{0 \pi} & \text {, if } b_{i .} \geq 0 \\
d_{i i}=1 & \text {,if } b_{i .} \geq 0
\end{array}
$$

These definitions imply:

$$
\left(B-\frac{1}{\rho} A\right)+C=D(B-\pi A)
$$

If $\rho \pi<1$ and if the LP-problem is p-directed (\$2.9), then $(8.2 \cdot 5)-$ mplies :

$$
c \geq 0
$$


With the help of these definitions, we find the following relation between system $(8.1 .1)$ and the problems $(8.2 .1)$, $(8.2 .2)$.

\section{3 Proposition.}

System ( 8.1 .1$)$ possesses a non-negative solution, if and only if a $q \in \mathrm{R}^{\mathrm{n}}$ exists, such that the corresponding problem $(8.2 .2)$ possesses an optimal ( $\left.u_{q}, v_{q}\right)$ which satisfies

$$
c^{\prime} u_{q}=q
$$

\section{Proof.}

Necessary: Let $(\tilde{x}, \tilde{y}),(\tilde{u}, \tilde{v})$ be a non-negative solution of $(8.1 .1)$ then $(\hat{x}, \hat{y}),(\hat{u}, \hat{v})$ satisfy:

$$
\begin{aligned}
& \ddot{v} \cdot \tilde{x}+\tilde{u} \cdot \tilde{y}=0 \\
& \left(B-\frac{1}{\rho} A\right)^{2} x+\frac{z}{y}=z \\
& \left(B^{\prime}-\frac{1}{p} A^{\prime}\right) D^{\prime} u_{-v}-\tilde{p}-C^{\prime} D^{-1} u \text {, , }
\end{aligned}
$$

in which the last equality is implied by $(8.2 .7)$. From this, by virtue of $8.2 .-b$, we may conclude: $(\bar{u}, \bar{v}):=\left(D^{*} \tilde{u}, \tilde{v}\right)$ is an optimal solution of problem (8.2.2) with $q=C^{\prime} \bar{u}$.

Sufficient: suppose that, for some $q \in R^{n}$, problem (8.2.2) possesses an optimal solution $\left(u_{q}, v_{q}\right)$ such that

$$
c^{\prime} u_{q}=q
$$

Then, by virtue of $8.2-a, b$, we may conclude that problem $(8.2 .1)$ possesses an optimal solution $\left(x_{q}, y_{q}\right) ; i . e .:$

$$
\begin{aligned}
& v_{q}^{\prime} x_{q}+u_{q}^{\prime} y_{q}=0 \\
& \left(B-\frac{1}{\rho} A\right) x_{q}+y_{q}=z
\end{aligned}
$$


Equality $(8.2 .7)$ implies

$$
\left(B^{\prime}-\frac{1}{\rho} A^{\prime}\right) u_{q}-v_{q}=\left(B^{\prime}-\pi A^{\prime}\right) D u_{q}-C^{\prime} u_{q}-v_{q}=\tilde{p}-q \text {, }
$$

and so, by virtue of $(8 \cdot 3.2)$ :

$$
\left(B^{\prime}-\pi A^{\prime}\right)\left(D u_{q}\right)-v_{q}=\tilde{p}
$$

Clearly, $\left(x_{q}, y_{q}\right),\left(D_{q}, v_{q}\right)$ is a non-negative solution of $(8.1 .1)$.

\subsection{Definition.}

The consequence of proposition 8.3 is, that instead of system ( 8.1 .1$)$ we may consider the linear programming problems $(8.2,1)$ and $(8.2 .2)$.

In order to investigate the existence of a $q \in \mathrm{R}^{\mathrm{n}}$ as mentioned in proposition 8.3 , we introduce the mapping $W: R^{n} \rightarrow \pi\left(R^{n}\right)$, defined by:

$$
W(q):=\left\{w=c^{\prime} u \mid u \in \underline{u}(q)\right\},
$$

where, $\underline{U}: R^{n} \rightarrow \pi\left(R^{m}\right)$ is defined in $8.2-c$.

\subsection{Proposition.}

If a set $Q$ exists which possesses the following properties:

a) $Q$ is compact and convex,

b) For every $q \in Q$, the problems $(8.2 .1),(8.2 .2)$ have feasible solutions $(x, y)>0,(u, v)>0$, resp.

c) $W(Q) \subset Q$,

then, system (8.1.1) possesses a non-negative solution.

\section{Proof.}

By virtue of $8.2-c$, supposition b implies that $\underline{U}: Q \rightarrow \Pi\left(R^{m}\right)$ is upper semicontinuous on $Q$. This implies $W: Q \rightarrow \pi\left(R^{n}\right)$ is 
upper semicontinuous as well.

The convexity of $\underline{U}(q)$ for every $q \in Q$ implies that $W(q)$ is convex for every $q \in Q$.

Thus, we have the following properties:

- $Q$ is compact and convex (by supposition a),

- for every $q \in Q$ : $W(q)$ non-empty and convex,

- $W: Q \rightarrow \pi\left(R^{n}\right)$ is upper semicontinuous,

- W(Q) C Q (by supposition $c$ ).

Then, Kakutani's fixed point theorem $(6: 67)$ implies the existence of $\hat{q}$ such that

$$
\hat{q} \in W(\hat{q}) \quad \text {. }
$$

In connection with the definition of $W$, this implies the existence of a $\hat{\mathbf{u}} \in \underline{\underline{U}}(\hat{q})$, such that $\mathrm{C}^{\prime} \hat{\mathbf{u}}=\hat{\mathrm{q}}$ and so, by virtue of proposition 8.3, the existence of a non-negative solution for $(8,1,1)$.

\subsection{Proposition.}

If: $C \geqq 0$ (def 8.2 .5$)$ and if the systems

$$
\left.\begin{array}{rl}
\left(B-\frac{1}{\rho} A+C\right) x+y & =z \\
x, y & >0
\end{array}\right\},
$$

are solvable, then the system (8.1.1) possesses a non-negative solution.

\section{Proof.}

We shall construct a set $Q \subset \mathbb{R}^{\mathrm{n}}$ which satisfies the conditions 
formulated in proposition 8.5 .

Let $(\underline{x}, \underline{y})$ be a solution of $(8.6 .1)$ then, for every $\left(q, u_{q}\right) \in \operatorname{graph}\left(R_{+}^{n} ; \underline{u}\right):$

$$
\begin{aligned}
\tilde{f}^{\prime} u_{q} & =\left(\left(B-\frac{1}{\rho} A+C\right) \underline{x}+\underline{y}\right)^{\prime} u_{q}= \\
& =\underline{x}^{\prime}\left(B^{\prime}-\frac{1}{\rho} A^{\prime}\right) u_{q}+\left(\underline{y}^{\prime}+\underline{x}^{\prime} C^{\prime}\right) u_{q} \geq \\
& \geq(\hat{p}-q)^{\prime} \underline{x}+\left(y_{-}^{\prime}+\underline{x}^{\prime} C^{\prime}\right) u_{q} .
\end{aligned}
$$

Let ( $\underline{u}, \underline{v})$ be a solution of $(8.6 .2)$, then we may conclude successively: $\underline{u}$ is a feasible solution of $(8.2 .2)$ if $q=0$; $\underline{u}$ is a

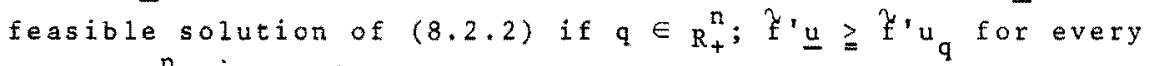
$u_{q} \in \underline{U}\left(R_{+}^{n}\right)$; and finally, by virtue of $(8.6 .3)$ :

$$
\underline{x}^{\prime} \underline{\underline{u}} \geq(\tilde{p}-q)^{\prime} \underline{x}+\left(\underline{y}^{\prime}+\underline{x}^{\prime} \mathrm{c}^{\prime}\right) u_{q},
$$

for every $\left(q, u_{q}\right) \in g r a p h\left(R_{+}^{n} ; \underline{U}\right)$.

Since $\underline{y}>0$, an $\varepsilon>0$ (small enough) exists such that $\underline{y}^{\prime}+\underline{x}^{\prime} \mathrm{C} \geqq(1+\varepsilon) \underline{x}^{\prime} C^{\prime}$. This implies by virtue of $(8.6 .4)$ :

$$
\underline{x}^{\prime} C^{\prime} u_{q} \leqq \frac{1}{1+\varepsilon} q^{\prime} \underline{x}+\frac{1}{1+\varepsilon}\left(z^{\prime} \underline{u}-\tilde{p}^{\prime} \underline{x}\right)
$$

for every $q, u_{q} \in \operatorname{graph}\left(\mathbb{R}_{+}^{n} ; \underline{U}\right)$.

Now define set $Q \subset R^{n}$ as follows:

$$
Q:=\left\{q \in R_{+}^{n} \mid \underline{x}^{\prime} q \leqq \frac{1}{\varepsilon}\left(\tilde{f} \cdot \underline{u}-\hat{p}^{\prime} \underline{x}\right)\right\} .
$$

Then (8.6.5) implies:

$$
\underline{x}^{\prime} C^{\prime} u_{q} \leqq \frac{1}{(1+\varepsilon) \varepsilon}\left(\tilde{f}^{\prime} \underline{u}_{-} \tilde{p}^{\prime} \underline{x}\right)+\frac{1}{1+\varepsilon}\left(\tilde{f}^{\prime} \underline{\underline{u}}-\tilde{p}^{\prime} \underline{x}\right)=\frac{1}{\varepsilon}\left(\tilde{f}^{\prime} \underline{u}-\tilde{p}^{\prime} \underline{x}\right),
$$

for every $\left(q, u_{q}\right) \in \operatorname{graph}(Q ;$ U $)$. 
Since $W: R^{n}+\Pi\left(R^{n}\right)$ is defined $(8.4 .1)$ by

$$
W(q):=\left\{w=c^{\prime} u \mid u \in \underline{U}(q)\right\},
$$

we may conclude from $(8.6 .6)$ and $(8.6 .7)$ :

$$
W(Q) \cap R_{+}^{n} \subset Q
$$

Since, by supposition, $C \geqq 0$ and since $\underline{U}(Q) \subset R_{+}^{\mathfrak{n}}$, definition (8.6.8) implies $W(Q) \subset R_{+}^{n}$. Hence, we may conclude

$$
W(Q) \subset Q
$$

Since $\underline{x}>0$ and since $\left(\hat{f^{\prime}} \underline{u}-\hat{p}^{\prime} \underline{x}\right)>0$ (from (8.6.4) by putting $q=0)$, definition $(8.6 .6)$ implies that $Q$ is compact, convex and non-empty. Moreover, since $Q \subset R_{+}^{n}$ and $C \geqq 0$, the solvability of $(8.6 .1)$ and $(8.6 .2)$ implies that the programing problems (8.2.1) and $(8.2 .2)$ possess-a feasible solution $(x, y)>0,(u, v)>0$ for every $q \in Q$. Hence, by virtue of proposition 8.5 , we may conclude: system (8.1.1) possesses a non-negative solution.

\section{7 Theorem.}

An LP-problem(P- or D-directed; $f(t)=\mathfrak{F}, p(t)=\stackrel{q}{\mathrm{p}}, t \geqq 1$; virtually superregular) possesses an equilibrium combination.

\section{Proof.}

Since the LP-problem is supposed to be initial strong regular and $\mathrm{p}$ - or D-directed, the systems

$$
\left.\begin{array}{r}
(B-\pi A) x+y=z \\
x, y>0
\end{array}\right\},
$$


are solvable $(3,10-e)$.

Now, suppose that the LP-problem is p-directed, then the definition of $\mathrm{P}$-directedness in $\$ 2.7$, the definition $(8.2 .6)$ of matrix $D$, and the inequality $\rho \pi<1$, imply:

$$
D \tilde{f} \leqq\left(\frac{1}{p \pi}\right) \tilde{f}
$$

Let $(\underline{x}, \underline{y})$ be a solution of $(8.7 .1)$, then:

$$
D(B-\pi A) \underline{x}+D y=D \hat{f} .
$$

Then, from inequality (8.7.3) and from equality (8.2.7), we may conclude:

$$
\left(B-\frac{1}{\rho} A+C\right) \underline{x}+D \underline{y} \leqq\left(\frac{1}{\rho \pi}\right) \tilde{f} .
$$

In connection with the definition of $D$, the latter inequality implies the solvability of the system

$$
\left.\begin{array}{rl}
\left(B-\frac{1}{\rho} A+C\right) x+y & =z \\
x, y & >0
\end{array}\right\} .
$$

Since $C \geqq 0(8.2 .8)$, and since the systems (8.7.2) and (8.7.4) are solvable, we may conclude, by virtue of proposition 8.6 , that the LP-problem possesses an equilibrium combination, in case p-directedness is supposed.

Now, suppose that the LP-problem is D-directed, then, if we write $(8.1 .1)$ :

$$
\left.\begin{array}{l}
\left(A^{\prime}-\frac{1}{\pi} B^{\prime}\right) \tilde{u}+\tilde{v}=\left(-\frac{1}{\pi} \tilde{p}\right) \\
\left(A-\rho B^{\prime}\right) \tilde{x}-\tilde{y}=(-\rho \tilde{f}) \\
\tilde{y} \cdot \tilde{u}+\tilde{x} \cdot \tilde{v}=0
\end{array}\right\},
$$

it is clear that the LP-problem also possesses an equilibrium combination. 


\section{SEMI-EQUILIBRIUM PATHS.}

\subsection{Introduction.}

Let $(\tilde{x}, \tilde{y}),(\tilde{u}, \tilde{v})$ be an equilibrium combination of an LP-problem (exponential), then every feasible solution $((x, y),(u, v)$ ) such that

$$
\left.\begin{array}{l}
(\tilde{v}, \tilde{u})^{\prime}(x(t), y(t))=0 \\
(\tilde{y}, \tilde{x})^{\prime}(u(t), v(t))=0
\end{array}\right\} \quad t \geq 1 \quad(9.1 .1)
$$

is called a semi-equilibrium path of $(\tilde{x}, \tilde{y}),(\tilde{u}, \tilde{v})$.

In accordance with $\$ 4.1$, we call a semi equilibrium path $((x, y),(u, v))$ consistent if simultaneously: $\left\{\langle p, x\rangle_{T}\right\}_{l}^{\infty}$, has a lower bound and $\left\{\left\langle f^{\circ}, u\right\rangle_{T}\right\}_{1}^{\infty}$ has an upper bound.

In this chapter, we shall especially investigate semi-equilibrium paths belonging to an equilibrium combination $(\tilde{x}, \tilde{y}),(\tilde{u}, \tilde{v})$ which possesses the following properties:

a) $(\tilde{x}, \tilde{y})$ contains exactly m positive components.

b) $(\tilde{u}, \tilde{v})$ contains exactly n positive components.

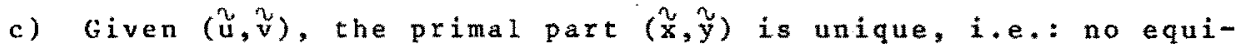
librium combination $(\underline{x}, \underline{y}),(\tilde{u}, \tilde{v}),(x, y) \neq(\tilde{x}, y)$ exists.

d) Given $(\tilde{x}, \tilde{y})$, the dual part $(\tilde{u}, \tilde{v})$ is unique, i.e.: no equilibrium combination $(\tilde{x}, \hat{y}),(\underline{u}, \underline{v}),(\underline{u}, \underline{v}) \neq(\tilde{u}, \underline{u})$ exists.

Such an equilibrium combination is called non-degenerated.

\subsection{Theorem.}

If $((x, y),(u, v))$ is a consistent semi equilibrium path of an LP-problem(P- or D-directed; exponential; virtually superregular) then, $x, y$ are $p$-dominated and $u, v$ are $\pi$-dominated.

\section{Proof.}

Let $((x, y),(u, v))$ be a consistent semi equilibrium path of an 
LP-problem(P- or D-directed; $f(t)=\tilde{f}, p(t)=\mathfrak{p}, t \geqq 1$; virtually superregular) belonging to an equilibrium combination $(x, y),(u, v)$. Then the following holds:

$$
\begin{aligned}
& \left(B^{\prime}-\pi A^{\prime}\right) \tilde{u}-\tilde{v}=\tilde{p}, \\
& \tilde{v}^{\prime} x(t)=0, t \geq 1, \\
& \ddot{u} \cdot y(t)=0, \quad t \geqq 1 \text {. } \\
& \left(B-\frac{1}{\alpha} A\right)\left(\frac{\alpha}{\rho}\right)^{T} \sum_{t=1}^{T-1}\left(\frac{1}{\alpha}\right)^{t} x(t)+B\left(\frac{1}{\rho}\right)^{T} x(T)+\left(\frac{\alpha}{\rho}\right)^{T} \sum_{t=1}^{T}\left(\frac{1}{\alpha}\right)^{t} y(t)= \\
& =\left(\frac{\alpha}{\rho}\right)^{\mathrm{T}}\left[\sum_{\mathrm{t}=1}^{\mathrm{T}}\left(\frac{\rho}{\alpha}\right)^{\mathrm{t}} \mathrm{t}+\frac{1}{\alpha} \mathrm{Ax}(0)\right], \mathrm{T} \geqq 1, \alpha>0, \\
& (9.2 .4) \\
& (B-\pi A)\left(\frac{1}{\rho \pi}\right)^{T} \quad \sum_{t=T+1}^{\infty} \pi^{t} x(t)-\pi A\left(\frac{1}{\rho}\right)^{T} x(T)+\left(\frac{1}{\rho \pi}\right)^{T} \sum_{t=T+1}^{\infty} \pi^{t} y(t)= \\
& =\left(\frac{1}{\rho \pi}\right)^{T} \sum_{t=T+1}^{\infty}(\rho \pi)^{t \eta} \quad, T \geqq 1
\end{aligned}
$$

where the latter inequality may be written by virtue of the fact that consistency implies $x_{\pi} \in 1_{1}^{n}, y_{\pi} \in 1_{1}^{m}$ (theorem 4.9).

We choose $\alpha \in 10$, ol in such a manner that

$$
\left(B^{\prime}-\frac{1}{\alpha} A^{\prime}\right) u-v=\hat{p}
$$

possesses a solution $u, v>0$. This is possible, since the LPproblem is supposed to be $P$ - or D-directed anc virtually super-. regular, which implies $(\$ 3.10)$ that

$$
\left.\begin{array}{rl}
\left(B^{\prime}-\frac{1}{\rho} A^{\prime}\right) u-v & =\tilde{p} \\
u, v & >0
\end{array}\right\}
$$


$-135-$

is solvable.

Now, suppose that $x, y$ are not $p$-dominated, then the sequence $\{n(T)\}_{1}^{\infty}$ defined by:

$$
\begin{aligned}
n(T): & =\left(\frac{\alpha}{\rho}\right)^{T} \sum_{t=1}^{T-1}\left(\frac{1}{\alpha}\right)^{t}\|x(t)\|_{1}+\left(\frac{1}{\rho}\right)^{T}\|x(T)\|_{1}+\left(\frac{1}{\rho \pi}\right)^{T} \sum_{t=T+1}^{\infty} \pi^{t}\|x(t)\|_{1}+ \\
& +\left(\frac{\alpha}{\rho}\right)^{T} \sum_{t=1}^{T}\left(\frac{1}{\alpha}\right)^{t}\|y(t)\|_{1}+\left(\frac{1}{\rho \pi}\right)^{T} \sum_{t=T+1}^{\infty} \pi^{t}\|y(t)\|_{1}, T \geqslant 1,
\end{aligned}
$$

contains a subsequence $\left\{\eta\left(T_{k}\right)\right\}_{k=1}^{\infty}$ such that

$$
n\left(T_{k+1}\right)>n\left(T_{k}\right)>0, \quad k \geq 1,
$$

and such that $\left\{n\left(T_{k}\right)\right\}_{k=1}^{\infty}$ has no upper bound.

When we define the sequences

$$
\begin{aligned}
& x^{1}(k):=\frac{1}{n\left(T_{k}\right)}\left(\frac{\alpha}{\rho}\right)^{T_{k}}{ }^{T_{k}} \sum_{t=1}^{-1}\left(\frac{1}{\alpha}\right)^{t} x(t) \quad, k \geqq 1, \\
& x^{2}(k):=\frac{1}{n\left(T_{k}\right)}\left(\frac{1}{p}\right)^{T_{k}} x\left(T_{k}\right), \quad, k \geqq 1, \\
& x^{3}(k):=\frac{1}{\eta\left(T_{k}\right)}\left(\frac{1}{\rho \pi}\right){ }^{T_{k}} \sum_{t=T_{k}+1}^{\infty} \pi^{t} x(t) \quad, k \geqq 1, \\
& y^{1}(k):=\frac{1}{\eta\left(T_{k}\right)}\left(\frac{\alpha}{p}\right)^{T} k \sum_{t=1}^{T_{1}} k\left(\frac{1}{\alpha}\right) y(t) \quad, k \geqq 1, \\
& y^{2}(k):=\frac{1}{\eta\left(T_{k}\right)}\left(\frac{1}{\rho \pi}\right) T_{k} \sum_{t=T_{k}+1}^{\infty} \pi^{t} y(t) \quad, k \geqq 1, \\
& f^{\prime}(k):=\frac{1}{\eta\left(T_{k}\right)}\left[\frac{1-(\alpha / \rho)^{T_{k}}}{1-\alpha / \rho}+\frac{1}{\alpha}\left(\frac{\alpha}{\rho}\right)^{T_{k}} A x(0)\right] k \geq 1 \text {, } \\
& f^{2}(k):=\frac{1}{\eta\left(T_{k}\right)} \frac{\rho \pi}{1-\rho \pi} F^{2}
\end{aligned}
$$


then it follows from $(9.2 .2)$ to $(9.2 .5)$, and from definition $(9.2 .7)$ that:

$$
\left.\begin{array}{l}
\left(B-\frac{1}{a} A\right) x^{1}(k)+B x^{2}(k)+y^{1}(k)=f^{1}(k) \\
(B-\pi A) x^{3}(k)-\pi A x^{2}(k)+y^{2}(k)=f^{2}(k) \\
x^{1}(k), x^{2}(k), x^{3}(k), y^{1}(k), y^{2}(k) \geq 0 \\
v^{\prime} x^{1}(k)=u^{\prime} x^{2}(k)=v^{\prime} x^{3}(k)=0 \\
u^{\prime} y^{1}(k)=u^{\prime} y^{2}(k)=0 \\
\left\|x^{1}(k)+x^{2}(k)+x^{3}(k)\right\|+\left\|y^{1}(k)+y^{2}(k)\right\|=1 \\
f^{1}(k) \rightarrow 0, \quad k+\infty, \\
f^{2}(k) \rightarrow 0, \quad k+\infty .
\end{array}\right\}
$$

This implies the existence of subsequences $\left\{x^{1}\left(k_{h}\right)\right\}_{h=1}^{\infty},\left\{x^{2}\left(k_{h}\right)\right\}_{h=1}^{\infty}$, $\left\{x^{3}\left(k_{h}\right)\right\}_{h=1}^{\infty},\left\{y^{1}\left(k_{h}\right)\right\}_{h=1}^{\infty}$, and $\left\{y^{2}\left(k_{h}\right)\right\}_{h=1}^{\infty}$ which converge to vectors $\underline{x}^{1}, \underline{x}^{2}, \underline{x}^{3}, y^{l}$ and $\underline{y}^{2}$ resp. satisfying:

$$
\begin{aligned}
& \left(B-\frac{1}{\alpha} A\right) \underline{x}^{1}+B \underline{x}^{2}+\underline{y}^{1}=0, \\
& (B-\pi A) \underline{x}^{3}-\pi A \underline{x}^{2}+\underline{y}^{2}=0, \\
& \underline{x}^{1}, \underline{x}^{2}, \underline{x}^{3}, \underline{y}^{1}, \underline{y}^{2} \geq 0, \\
& \left\|\underline{x}^{1}+\underline{x}^{2}+\underline{x}^{3}\right\|+\left\|\underline{y}^{1}+\underline{y}^{2}\right\|=1, \\
& \tilde{v}^{\prime} \underline{x}^{1}=\tilde{v}^{\prime} \underline{x}^{2}=\tilde{v}^{\prime} \underline{x}^{3}=0, \\
& \tilde{u}^{\prime} \underline{y}^{1}=\tilde{u}^{\prime} \underline{y}^{2}=0 .
\end{aligned}
$$

The equalities $(9.2 .9)$ and $(9.2 .10)$ imply 


$$
(B-\pi A)\left(\underline{x}^{1}+\underline{x}^{2}+\underline{x}^{3}\right)+\left(\underline{y}^{1}+\underline{y}^{2}\right)=\left(\frac{1}{\alpha}-\pi\right) A \underline{x}^{1} .
$$

The equalities $(9.2 .1),(9.2 .15),(9.2 .13)$ and $(9.2 .14)$ imply:

$$
\underline{p}^{2}\left(\underline{x}^{1}+\underline{x}^{2}+\underline{x}^{3}\right)=\left(\frac{1}{\alpha}-\pi\right) u^{2} \cdot \underline{x}^{1} .
$$

In case the LP-problem is P-directed (9.2.9) implies:

$$
A \underline{x}^{1} \geq 0
$$

In case the LP-problem is D-directed $(9.2 .1)$ implies:

$$
A \cdot u+v \geq 0
$$

Since $\tilde{v}^{\prime} \underline{x}^{1}=0$, the inequalities $(9.2 .17)$ and $(9.2 .18)$ both imply:

$$
\mathfrak{u}^{\prime} \cdot x^{\prime} \geq 0 .
$$

Since $\frac{1}{\alpha}>\pi$, it follows from $(9.2 .16)$ and from $(9.2 .19)$ that:

$$
\tilde{p}^{\prime}\left(\underline{x}^{1}+\underline{x}^{2}+\underline{x}^{3}\right) \geqq 0
$$

Now, suppose that the LP-problem is P-directed. Then, equality $(9.2 .10)$ and $\left(\frac{1}{\alpha}-\pi\right)>0$ imply:

$$
-\left(\frac{1}{a}-\pi\right) A\left(\underline{x}^{2}+\underline{x}^{3}\right) \leqq 0
$$

Adding $(9.2 .9),(9.2 .10)$ and $(9.2 .21)$ we find:

$$
\left(B-\frac{1}{\alpha} A\right)\left(\underline{x}^{1}+\underline{x}^{2}+\underline{x}^{3}\right)+\left(\underline{y}^{1}+\underline{y}^{2}\right) \leqq 0
$$

Then, by virtue of stiemkes theorem (page 31), it follows from $(9.2 .20),(9.2 .21),(9.2 .12)$, and $(9.2 .11)$ that system

$$
\left.\begin{array}{rl}
\left(B-\frac{1}{\alpha} A\right) u-v & \tilde{p} \\
u, v & >0
\end{array}\right\}
$$


is non-solvable. However, $\alpha$ is chosen in such a manner that (9.2. 6) possesses a solution $u, v>0$. Thus, the suppositions that the LP-problem is $P$-directed and that $x, y$ are not $\rho^{-}$ dominated, give $r$ ise to contradictoriness.

Now, consider the case that the LP-problem is D-directed.

The equalities $(9.2 .9)$ and $(9.2 .10)$ imply:

$$
\left.\left(B-\frac{1}{\alpha} A\right) \underline{x}^{1}+(B-\pi A) \underline{x}^{2}+\underline{x}^{3}\right)+\left(\underline{y}^{1}+\underline{y}^{2}\right)=0
$$

By virtue of Stiemke's theorem, it follows from (9.2.22), $(9.2 .20) .(9.2 .11)$, and from $(9.2 .12)$, that the system

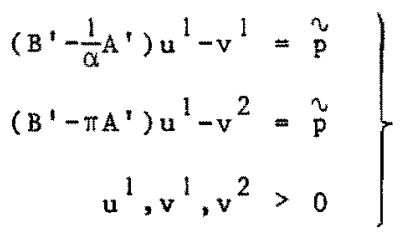

is non-solvable. However, if $(u, v)>0$ is a solution of $(9.2 .6)$ then $D$-directedness implies that $u^{1}:=u, v^{1}:=v$, $v^{2}:=v+\left(\frac{1}{\alpha}-\pi\right) A^{\prime} u$, is a solution of (9.2.23). Hence, the suppositions that the LP-problem is $\mathrm{D}-\mathrm{d}$ irected and that $x, y$ are not p-dominated give rise to contradictoriness.

The theorem with respect to the dual part of a semi-equilibrium path, follows from the validity of the theorem for the primal part and from the symmetry between the primal and dual systems.

\subsection{Definitions.}

From now on, we shall consider semi-equilibrium paths belonging to a non-degenerated equilibrium combination.

The definition of a non-degenerated equilibrium combination $(x, y),(\tilde{u}, \tilde{v})$ implies:

- components $x$; and $\tilde{v}$; cannot be simultaneously zero,

- components $\tilde{u}$; and $\tilde{y}$; cannot be simultaneously zero. 
This implies the following relations:

- if $\ddot{x}$ possesses $k$ positive components, then:

- y has (m-k) positive components,

- u has k positive components,

- $v$ has $(n-k)$ positive components.

We define the following quantities:

a) $n \times k$-matrix $s_{p}$, consisting of the column vectors of the nxn-identity matrix which correspond in the same order with the positive components of $\mathrm{x}$,

b) kxm-matrix $s_{d}$, consisting of the row vectors of the mxm-identity matrix which correspond in the same order with the positive components of $u$,

c) The $k \times k$ matrices $\underline{A}$ and $\underline{B}$ by:

$$
\begin{aligned}
& \underline{A}:=S_{d} A S_{p} \\
& \underline{B}:=S_{d} B S_{p}
\end{aligned}
$$

d) The sets $X Y Y^{n} \subset 1^{n} \times 1^{m}$ and Ư $\subset 1^{m} \times 1^{n}$ by:

$$
\begin{aligned}
& \tilde{X} \tilde{Y}:=\left\{(x, y) \in 1^{n_{\times 1} m} \mid \begin{array}{ll}
x(t)=s_{p} \underline{x}(t), & t \geqq 1, \underline{x} \in 1^{k} \\
s_{d} y(t)=0, & t \geqq 1
\end{array}\right\}, \\
& \tilde{U} \tilde{V}:=\left\{(u, v) \in 1^{m_{\times 1} n} \mid \begin{array}{ll}
u(t)=s_{d}^{\prime} \underline{u}(t), & t \geqq 1, \underline{u} \in 1^{k} \\
s_{p}^{\prime} v(t)=0, & t \geqq 1
\end{array}\right\} .
\end{aligned}
$$

Now, vectors $(\underline{x}, v) \in 1^{k} \times 1^{k}$ and $((x, y),(u, v)) \in \hat{X} Y \times Y \mathfrak{V}$ will be considered, satisfying the following systems:

$$
\left.\begin{array}{l}
B x(t+1)-A x(t)+y(t+1)=\rho^{t+1} \tilde{z} \\
B^{\prime} u(t)-A^{\prime} u(t+1)-v(t)=\pi{ }_{p}^{t \tilde{p}}
\end{array}\right\} t \geq 1,
$$




$$
\left.\begin{array}{l}
B \times(t+1)-A x(t)=e^{t+1} s_{d}^{z} \\
\underline{B}^{\prime} \underline{u}(t)-\underline{A}^{\prime} \underline{u}(t+1)=\pi^{t} s_{p}^{\prime} \tilde{p}
\end{array}\right\} \quad t \geq 1 .
$$

\subsection{Proposition.}

If $(\tilde{x}, \tilde{y}),(\tilde{u}, \tilde{v})$ is a non-degenerated equilibrium combination, then the corresponding systems $(9.3 .1),(9.3 .2)$, and corresponding sets $X_{Y}, \mathcal{Y}$, possess the following properties:

a) A vector $((x, y),(u, v)) \in\left(1^{n} \times 1^{m}\right) \times\left(1^{m} \times 1^{n}\right)$ satisfies:

$$
\left.\begin{array}{l}
x(t), y(t), u(t), v(t) \geqq 0 \\
(\tilde{v}, \tilde{u}) \cdot(x(t), y(t))=0 \\
(\tilde{y}, \tilde{x})^{\prime}(u(t), v(t))=0
\end{array}\right\} \quad t \geq 1
$$

if and only it

$$
((x, y),(u, v)) \in\left(\hat{X Y} \cap 1_{+}^{n} \times 1_{+}^{m}\right) \times\left(\hat{y} \cap 1_{+}^{m} \times 1_{+}^{n}\right)
$$

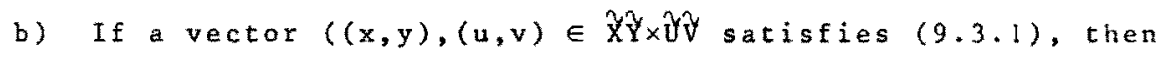
$(\underline{x}, \underline{u})$ defined by

$$
\left.\begin{array}{l}
\underline{x}(t):=s_{p}^{\prime} x(t) \\
\underline{u}(t):=s_{d} u(t)
\end{array}\right\} t \geqq 1,
$$

satisfies $(9.3 .2)$.

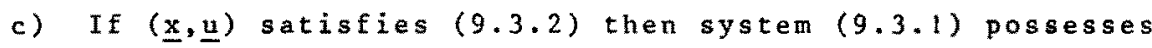
a solution $((x, y),(u, v))$, satisfying $(9.3 .1)$ and:

$$
\left.\begin{array}{l}
x(t)=s_{p} \underline{x}(t) \\
u(t)=s_{d}^{\prime} \underline{u}(t)
\end{array}\right\} \quad t \geq 1
$$

Moreover, $((x, y),(u, v)) \in \hat{X} \hat{Y} \times \hat{U} \hat{V}$. 


\section{Proof.}

(a) Necessary: If $((x, y),(u, v))$ satisfies $(9.4 .1)$, then the non-degeneratedness of $(\tilde{x}, \tilde{y}),(\tilde{u}, \tilde{v})$ implies that all components of $(x(t), y(t))_{r}$, and $(u(t), v(t))_{s}, t \geqq 1$, corresponding. with components $(\tilde{x}, \tilde{y})_{r}=0,(\tilde{u}, \tilde{v})_{s}=0$ resp., are zero. Then, definition 9.3-a,b implies:

$$
\left.\begin{array}{l}
x(t)=s_{p} s_{p}^{\prime} x(t) \\
s_{d} y(t)=0 \\
u(t)=s_{d}^{\prime} S_{d} u(t) \\
S_{p}^{\prime} v(t)=0
\end{array}\right\} t \geqq 1 .
$$

From (9.4.5), 9.3-d, and from the non-negativity of $x, y$, $u, v$, we may conclude that $(9.4 .2)$ is valid.

(a) Sufficient: Definition $9.3-d$ implies that $\tilde{v}^{\prime} \mathrm{s}_{\mathrm{p}}=0^{\prime}$ and $s_{d} \tilde{y}=0$. Hence, we may conclude that for all vectors $(x, y) \in X_{Y} Y$ and $(u, v) \in \mathcal{U} Y$ :

$$
\left.\begin{array}{l}
(\tilde{v}, \tilde{u}) \cdot(x(t), y(t))=0 \\
(\tilde{y}, \tilde{x})^{\prime}(u(t), v(t))=0
\end{array}\right\} t \geqq 1 .
$$

(b) The definitions $9.3-a, b, d$ imply that all vectors $(x, y) \in \tilde{X} Y$ and $(u, v) \in \tilde{U} \tilde{V}$ satisfy (9.4.5). This implies for every $((x, y),(u, v)) \in \mathcal{X} Y \times Y U Y$ which satisfies $(9 \cdot 3.1)$ :

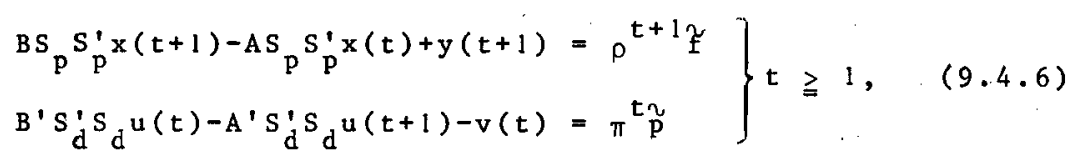

and so,

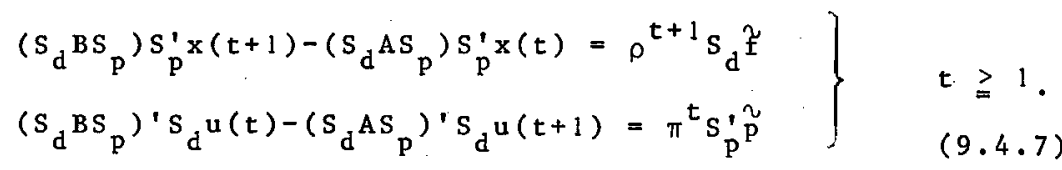




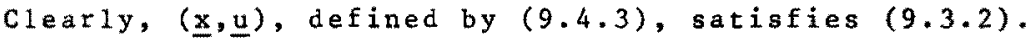

(c) If $(\underline{x}, \underline{u})$ satisfies $(9.3 .2)$, then $(x, u)$ defined by $(9.4 .4)$ satisfies:

$$
\left.\begin{array}{l}
s_{d} B x(t+1)-S_{d} A x(t)=\rho^{t+1} s_{d} \tilde{f} \\
S_{p}^{\prime} B^{\prime} u(t)-S_{p}^{\prime} A^{\prime} u(t+1)=\pi^{t} s_{p}^{n}
\end{array}\right\} \quad t \geqq 1 .
$$

Clearly, for this unique $(x, u)$, a unique $(y, v)$ exists such that $((x, y),(u, v))$ satisfies $(9.3 .1)$. Moreover, $(9.4 .8)$ implies:

$$
\left.\begin{array}{l}
s_{d} y(t)=0 \\
s_{p}^{\prime} v(t)=0
\end{array}\right\} .
$$

From $(9.4 .4)$ and from $(9.4 .9)$, we may concluce that

$$
((x, y),(u, v)) \in \hat{X} Y \times Y \hat{U}
$$

\subsection{Proposition.}

If $(\hat{x}, \tilde{y}),(\hat{u}, \vec{v})$ is a non-degenerated equilibrium combination, then the matrices $\left(\underline{B}-\frac{1}{\rho} A\right)$ and $(\underline{B}-\pi \underline{A})$ defined by $9.3-c$ are both invertible.

\section{Proof.}

Suppose that matrix ( $\left.B-\frac{1}{p} A\right)$, belonging to a non-degenerated equilibrium combination $(\hat{x}, \hat{y}),(\tilde{u}, \tilde{v})$, is not invertible. Then a vector $\underline{x} \neq 0$ exists such that

$$
\left(\underline{B}-\frac{1}{p} A\right) \underline{x}=0 .
$$

This implies that, for every $\lambda \in \mathrm{R}^{1}$ :

$$
S_{d}\left(B-\frac{1}{\rho} A\right)\left(\tilde{x}+\lambda S_{p} x\right)=s_{d} z
$$


Then, from the definitions $9.3-a, b$, we may conclude that $a$ vector $y$ exists, such that for every $\lambda$ :

$$
\left.\begin{array}{l}
\left(B-\frac{1}{\rho} A\right)\left(\tilde{x}+\lambda s_{p} x\right)+(\tilde{y}+\lambda \underline{y})=\tilde{f} \\
(\tilde{v}, \tilde{u}) \cdot\left(\tilde{x}+\lambda s_{p} x, \tilde{y}+\lambda y\right)=0
\end{array}\right\} .
$$

Since $(\tilde{x}, \tilde{y}),(\tilde{u}, \tilde{v})$ is non-degenerated, the definitions $9,3-a, b$ imply:

- for every component ${\underset{\sim}{x}}_{j}=0:\left(s_{p} x_{j}=0\right.$,

- for every component $y_{i}=0: \underline{y}_{i}=0$.

In connection with the non-negativity of $(\stackrel{v}{x}, \tilde{y}),(\tilde{u}, \tilde{v})$, this implies the existence of a $\lambda \neq 0$ (small enough) such that

$$
\left(x^{2}+\lambda s p x\right),(y+\lambda y) \geq 0
$$

From (9.5.2) and $(9.5 .3)$, we may conclude that $\left(\hat{x}+\lambda s_{p} x, y+\lambda y\right),(\hat{u}, \hat{v})$ is an equilibrium combination. Since $\lambda s_{p} \neq 0$, we find that this conclusion is contradictory with respect to the supposition that $(\hat{x}, \tilde{y}),(\tilde{u}, \tilde{v})$ is non-degenerated. Thus we may conclude that $\left(\underline{B}-\frac{1}{p} A\right)$ is invertible.

In a similar manner, one may prove that (B-TA) is invertible.

\subsection{Definitions.}

From proposition 9.4 we may conclude that all semi-equilibrium paths belonging to a non-degenerated equilibrium combination are determined by solutions $(\underline{x}, \underline{u})$ of a system (9.3.2). For this reason, we now shall investigate system (9.3.2).

Every solution of $(9.3 .2)$ can be written as the sum of a particular solution of $(9.3 .2)$ and a solution $\left\{x^{\circ}(t)\right\}_{1}^{\infty},\left\{u^{\circ}(t)\right\}_{1}^{\infty}$ of its homogeneous form:

$$
\left.\begin{array}{l}
\underline{B x}^{\circ}(t+1)=\underline{A x}^{\circ}(t) \\
\underline{B}^{\prime} u^{\circ}(t)=\underline{A}^{0} u(t+1)
\end{array}\right\} t \geq 1,
$$


One may verify that

$$
\left.\begin{array}{l}
\bar{x}(t)=\rho^{t} s_{p}^{i} \tilde{x} \\
\bar{u}(t)=\pi^{t} s_{d} \tilde{u}
\end{array}\right\}, t \geqq 1
$$

is a particular solution of $(9.3 .2),(\tilde{x}, \tilde{y}),(\tilde{u}, \tilde{v})$ being the nondegenerated equilibrium combination.

Now, we shall investigate system $(9.6 .1)$. First, we remark that it is everyway possible that none of the matrices $A$ and $\underline{B}$ is invertible. However, the definition of a non-degenerated equilibrium combination implies (prop. 9.5) that the matrices $\left(\underline{B}-\frac{1}{p} \underline{A}\right)$ and $(\underline{B}-\pi \underline{A})$ are both invertible.

Exploiting the fact that $(\underline{B}-\pi \underline{A})^{-1}$ exists, system $(9.6 .1)$ can be transformed into:

$$
\left.\begin{array}{l}
(\underline{B}-\pi \underline{A})^{-1} \underline{B x}^{0}(t+1)=(\underline{B}-\pi \underline{A})^{-1} \underline{A} x(t) \\
\underline{B}^{\prime}\left(\underline{B}^{\prime}-\pi A^{\prime}\right)^{-1}\left(\underline{B}-\pi A^{\prime}\right) u^{0}(t)=\underline{A}^{\prime}(\underline{B}-\pi \underline{A})^{-1}(\underline{B}-\pi \underline{A}) u^{0}(t+1)
\end{array}\right\} t \geq 1 .
$$

When we define

$$
H:=(\underline{B}-\pi \underline{A})^{-1} \underline{B},
$$

which inplies:

$$
(\underline{B}-\pi \underline{A})^{-1} \underline{A}=\frac{1}{\pi}(H-I),
$$

then $(9.6 .3)$ can be reduced to:

$$
\left.\begin{array}{l}
H x^{\circ}(t+1)=\frac{1}{\pi}(H-I) x^{o}(t) \\
H^{\prime}\left(\underline{B}^{\prime}-\pi \underline{A}^{\prime}\right) u^{\circ}(t)=\frac{1}{\pi}(H-I)^{\prime}\left(\underline{B}^{\prime}-\pi \underline{A}^{\prime}\right) u^{0}(t+1)
\end{array}\right\} t \geq 1
$$


Now, let $\Lambda$ be a lower Jordan matrix (3:152) of H, and $Q$ a corresponding non-singular complex matrix, such that ll can be written:

$$
H=Q \wedge Q^{-1} \text {. }
$$

Then, system $(9.6 .6)$ can be transformed into the following systems:

$$
\begin{array}{ll}
\Lambda z(t+1)=\frac{1}{\pi}(\Lambda-I) z(t), & t \geqq 1, \\
\Lambda^{\prime} w(t)=\frac{1}{\pi}\left(\Lambda^{\prime}-I\right) w(t+1), & t \geqq 1, \\
x^{0}(t)=Q z(t), & t \geqq 1, \\
\left(\underline{B}^{\prime}-\pi \underline{A}^{\prime}\right) \mathrm{u}^{0}(t)=\left(Q^{-1}\right) \cdot w(t), & t \geqq 1 .
\end{array}
$$

We observe that eigenvalues $\lambda_{i}$, column vectors of $Q$, and row vectors of $\mathrm{Q}^{-1}$, with a non-zero imaginary part, always appear in pairs which are conjugate complex; i.e., a permutation matrix S exists such that

$$
\left.\begin{array}{l}
Q S=\operatorname{conj}(Q):=\operatorname{Re}(Q)-i \operatorname{Im}(Q) \\
S^{-1} Q^{-1}=\operatorname{conj}\left(Q^{-1}\right):=\operatorname{Re}\left(Q^{-1}\right)-i \operatorname{Im}\left(Q^{-1}\right) \\
S^{-1} \Lambda S=\operatorname{conj}(\Lambda):=\operatorname{Re}(\Lambda)-i \operatorname{Im}(\Lambda)
\end{array}\right\},
$$

where: $Q=\operatorname{Re}(Q)+i \operatorname{Im}(Q), Q^{-1}=\operatorname{Re}\left(Q^{-1}\right)+i \operatorname{Im}\left(Q^{-1}\right)$, and $\Lambda=\operatorname{Re}(\Lambda)+i \operatorname{Im}(\Lambda)$.

In the following theorems, solutions $\left\{x^{\circ}(t)\right\}_{1}^{\infty},\left\{u^{\circ}(t)\right\}_{1}^{\infty}$ of (9.6.1) for which

$$
\begin{aligned}
& \left(\frac{1}{\rho}\right)^{t} x^{o}(t)+0, t \rightarrow \infty, \\
& \left(\frac{1}{\pi}\right)^{t} u^{o}(t)+0, t \rightarrow \infty,
\end{aligned}
$$

will play a central role. 
In that context, it will be convenient to define:

- the spaces of k-dimensional complex vectors:

$$
\begin{aligned}
& z:=\left\{z \mid z_{i}=0, \text { if } \lambda_{i i}=0 \text { or }\left|\frac{\lambda_{i i}-1}{\lambda_{i i}}\right| \geqq p \pi\right\}, \\
& W:=\left\{w \mid w_{i}=0, \text { if } \lambda_{i i}=1 \text { or }\left|\frac{\lambda_{i i}}{\lambda_{i i}-1}\right| \geqq 1\right\},
\end{aligned}
$$

- the spaces of sequences of k-dimensional complex vectors:

$$
\begin{aligned}
& \underline{z}:=\left\{\begin{array}{l|l}
\{z(t)\}_{1}^{\infty} & \begin{array}{l}
\Lambda z(t+1)=\frac{1}{\pi}(\Lambda-I) z(t), t \geq 1 \\
\left(\frac{1}{p}\right)^{t} z(t)-0, t \rightarrow \infty
\end{array}
\end{array}\right\}, \\
& W:=\left\{\begin{array}{l|l}
\{w(t)\}_{1}^{\infty} & \begin{array}{l}
\Lambda^{\prime} w(t)-\frac{1}{\pi}\left(\Lambda^{+}-I\right) w(t+1), t \geq 1 \\
\left(\frac{1}{\pi}\right)^{t} w(t) \rightarrow 0, t \rightarrow \infty
\end{array}
\end{array}\right\},
\end{aligned}
$$

- the spaces of k-dimensional real vectors:

$$
\begin{aligned}
& x^{0}:=\left\{x \in R^{k} \mid x=Q z, \text { for some } z \in z\right\}, \\
& U^{0}:=\left\{u \in R^{k} \mid u=\left(Q^{-1}\right)^{\prime} w \text {, for some } w \in W\right\},
\end{aligned}
$$

- the subspaces of $1^{k}$

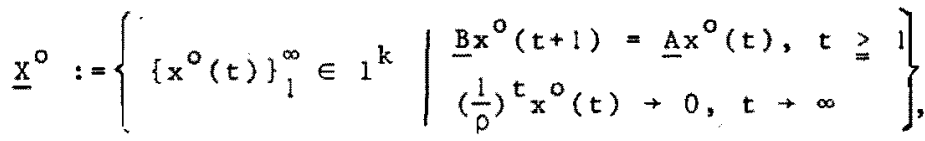

$$
\begin{aligned}
& \underline{u}^{0}:=\left\{\begin{array}{l|l}
\left\{u^{0}(t)\right\}_{1}^{\infty} \in I^{k} & \begin{array}{l}
\underline{B}^{\prime} u(t)=A^{\prime} u(t+1), t \geqq 1 \\
\left(\frac{1}{\pi}\right)^{t} u^{0}(t) \rightarrow 0, t+\infty
\end{array}
\end{array}\right\},
\end{aligned}
$$

- the subspaces of $\hat{X} Y$ and $\mathcal{V} \hat{V}$ (viz. def. 9.3-d): 
$\underline{X Y}:=\left\{\begin{array}{l|l}(x, y) \in X Y & \begin{array}{l}B x(t+1)-A x(t)+y(t+1)=\rho^{t+1} \hat{f} \\ \left(\frac{1}{p}\right)^{t}(x(t), y(t))+(\hat{x}, \hat{y}), t \rightarrow \infty\end{array}\end{array}\right\}(9.6 .21)$ $\underline{\Psi V}:=\left\{\begin{array}{ll}(u, v) \in \forall \forall & \begin{array}{l}B^{\prime} u(t)-A^{\prime} u(t+1)-v(t)=\pi^{t \tilde{p}} \\ \left(\frac{1}{\pi}\right)^{t}(u(t), v(t)) \rightarrow(\tilde{u}, v), t \rightarrow \infty\end{array}\end{array}\right\}(9.6 .22)$

\subsection{Proposition.}

a) If a sequence of k-dimensional complex vectors $\{z(t)\}_{1}^{\infty}$ satisfies $(9.6 .8)$, then:

$$
z_{i}(t)=0, t \geq 1 \text {, if } \lambda_{i i}=0
$$

b) If a sequence of k-dimensional complex vectors $\{w(t)\}_{1}^{\infty}$ satisfies $(9.6 .9)$, then:

$$
w_{i}(t)=0, t \geq 1 \text {, if } \lambda_{i i}=1 \text {. }
$$

c) If $\{z(t)\}_{1}^{\infty} \in \underline{z}$, then:

$$
z(t) \in Z, t \geqq 1,
$$

i.e.: $z_{i}(t)=0$, $t \geqq 1$, if $\lambda_{i i}=0$ or $\left|\left(\lambda_{i i}-1\right) / \lambda_{i i}\right| \geqq \rho \pi$.

d) If $\{w(t)\}_{1}^{\infty} \in \underline{w}$, then:

$$
\begin{aligned}
& w(t) \in w, t \geqq 1 \\
& \text { i.e.: } w_{i}(t)=0, t \geqq 1, \text { if } \lambda_{i i}=1 \text { or }\left|\lambda_{i i} /\left(\lambda_{i i}-1\right)\right| \geqq 1 .
\end{aligned}
$$

e) If $\bar{z} \in Z$, then one and only one sequence $\{z(t)\}_{1}^{\infty} \in \underline{Z}$ exists such that

$$
z(1)=\bar{z} \text {. }
$$


f) If $\bar{w} \in W$, then one and only one sequence $\{w(t)\}_{1}^{\infty} \in \underline{w}$ exists such that

$$
w(1)=\bar{w} .
$$

g) Positive numbers $\tilde{\rho}<\rho$ and $N_{1}$ exist such that every $\{z(t)\}_{l}^{\infty} \in \underline{z}$ satisfies:

$$
\|z(t+T)\|_{\infty} \leqq \tilde{\rho}^{t} N_{1}\|z(t)\|_{\infty}, t \geqq 0, T \geqq 1
$$

h) Positive numbers $\tilde{\pi}<\pi$ and $N_{2}$ exist such that every $\{w(t)\}_{1}^{\infty} \in \underline{W}$ satisfies:

$$
\|w(t+T)\|_{\infty} \leqq \pi^{t} N_{2}\|w(T)\|_{\infty}, t \geqq 0, T \geqq 1 .
$$

\section{Proof.}

(a) Suppose that $\Lambda$ is diagonal. Then system $(9.6 .8)$ can be written

$$
\lambda_{i i} z_{i}(t+1)=\frac{1}{\pi}\left(\lambda_{i i}-1\right) z_{i}(t), i=1, \ldots, k, t \geqq 1 .(9.7 .1)
$$

Clearly, if $\{z(t)\}_{1}^{\infty}$ satisfies $(9.6 .8)$, then:

$$
z_{i}(t)=0, t \geqq 1, \text { if } \lambda_{i i}=0
$$

Suppose that $\Lambda$, which is defined as a lower Jordan matrix, contains a Jordan block. Then the corresponding equalities of $(9.6 .8)$ may be written:

$$
\left.\begin{array}{cc}
\lambda z_{i}(t+1) & =\frac{1}{\pi}(\lambda-1) z_{i}(t) \\
z_{i}(t+1) & +\lambda z_{i+1}(t+1)=\frac{1}{\pi} z_{i}(t)
\end{array} \quad+\frac{1}{\pi}(\lambda-1) z_{i+1}(t)\right\} t \geq 1,
$$


where $\lambda$ is the eigenvalue of the Jordan block.

Now, suppose that $\lambda=0$. Then the first equality of $(9.7 .3)$ implies: $z_{i}(t)=0, t \geq 1$. And so, by virtue of the second equality of $(9.7 .3): z_{i+1}(t)=0, t \geqq 1$. Repeating this process; we find:

$$
\left.\begin{array}{l}
z_{i}(t)=0 \\
z_{i+j}(t)=0
\end{array}\right\}, t \geq 1 .
$$

Hence we may conclude that also in case $\Lambda$ is not diagonal, the equalities hold.

(b) This property can be proved in a similar manner as 9.7-a.

(c) We first assume that $\Lambda$ is diagonal. Then (9.7.1) and (9.7.2) imply that $(9.6 .8)$ can be written:

$$
\left.\begin{array}{cc}
\left(\frac{1}{\rho}\right)^{t+1} z_{i}(t+1)=\left(\frac{\lambda_{i i}-1}{\rho \pi \lambda_{i i}}\right)^{t}\left(\frac{1}{\rho}\right) z(1), & \text { if } \lambda_{i i} \neq 0 \\
z_{i}(t)=0, & \text { if } \lambda_{i i}=0
\end{array}\right\}, t \geq 1
$$

clearly, $\left(\frac{1}{\rho}\right)^{t} z(t) \rightarrow 0, t \rightarrow \infty$, implies

$$
z_{i}(t)=0, t \geqq 1, \text { if } \lambda_{i i}=0 \text { or }\left|\frac{\lambda_{i i}-1}{\lambda_{i i}}\right| \geqq 0 \pi
$$

In connection with the definitions $(9.6 .13)$ and $(9.6 .15)$, we may conclude that $\{z(t)\}_{1}^{\infty} \in \underline{z}$ implies $z(t) \in z, t \geqq 1$, in case $\Lambda$ is diagonal. In the case that $\Lambda$ is not diagonal, the validity of this property can be proved in a similar manner as done for 9.7-a.

(d) This property can be proved in a similar manner as 9.7-c.

(e) First we observe that for every vector $z^{1} \in z$ a unique vector $z^{2} \in z$ exists such that: 


$$
\Lambda z^{2}=\frac{1}{\pi}(\Lambda-T) z^{1}
$$

This implies that for every $z(1) \in Z$ a unique sequence $(z(t)\}_{2}^{\infty}$ exists such that

$$
\left.\begin{array}{l}
\Lambda z(t+1)=\frac{1}{\pi}(\Lambda-I) z(t) \\
z(t+1) \in z
\end{array}\right\} t \geqq 1 .
$$

When we define the matrix $\Lambda$ by:

$$
\left.\begin{array}{l}
\lambda_{i j}:=\lambda_{i j}, i, j=1, \ldots, k, i \neq j \\
\left.\lambda_{i i}:=\lambda_{i i} \text {, if } \lambda_{i i} \neq 0 \text {, and }\left|\frac{\lambda_{i i}-1}{\lambda_{i i}}\right|<0 \pi\right\} \\
\lambda_{i i}:=1, \text { in the other case }
\end{array}\right\}
$$

and the matrix $A$ by:

$$
\Lambda:=\frac{1}{\pi} \Lambda^{-1}(\Lambda-I)
$$

(note: matrix $\Lambda$ is invertible), then the definition of $z$ $(9.6 .13)$ implies that $(9.7 .5)$ can be written:

$$
z(t+1)=A z(t), t \geqq 0 ; z(1) \in z
$$

One may verify that $A$ is triangular with diagonal elements:

$$
\begin{aligned}
& \left.\lambda_{i i}=\frac{1}{\pi} \frac{\lambda_{i i}-1}{\lambda_{i i}}, \text { if } \lambda_{i i} \neq 0 \text { and } \frac{1}{\pi}\left|\frac{\lambda_{i i}-1}{\lambda_{i i}}\right|<\rho\right\} \\
& \lambda_{i i}=0, \text { in the other case }
\end{aligned}
$$

This implies that the $\lambda_{i}$ in $(9.7 .9)$ are the eigenvalues of A, so that:

$$
\bar{\lambda}:=\max _{i}\left|\lambda_{i i}\right|<\rho .
$$


Since $(*)$ for al1 $\delta>0$ a number $N_{\delta}$ exists for which

$$
\left\|\Lambda^{t}\right\|_{\infty} \leqq N_{\delta}(\bar{\lambda}+\delta)^{t}, t \geqq 0
$$

we may conclude that numbers $\tilde{p} \in 1 \bar{\lambda}, p l$ and $N_{1}>0$ exist such that every sequence $\{z(t)\}_{1}^{\infty}$ which satisfies $(9.7 .5)$ also satisfies

$$
\|z(t+T)\|_{\infty} \leqq \rho^{t} N_{1}\|z(T)\|_{\infty}, t \geqq 0, T \geqq 1 . \quad(9.7 .10)
$$

Since $\tilde{\rho} \in] 0, o l$, this implies:

$$
\left(\frac{1}{\rho}\right)^{t} z(t) \rightarrow 0, t \rightarrow \infty \text {. }
$$

From $(9.7 .5),(9.7 .11)$, and from the definition of $\underline{Z}(9.6 .15)$, we may conclude: $\{z(t)\}_{1}^{\infty} \in \underline{z}$. Since (9.7.8) implies the uniqueness of $\{z(t)\}_{2}^{\infty}$, given $z(1)$, we may conclude that $9.7-e$ has been proved.

(g) Since every $\{z(t)\}_{1}^{\infty}$ satisfies (9.7.6), this property is implied by $(9,7,10)$.

(f) and ( $h$ ): These properties can be proved in a similar manner as $9.7-\mathrm{e}$ and $9.7-\mathrm{g}$.

\subsection{Proposition.}

a) If a sequence of $k$-dimensional real vectors $\left\{x^{0}(t)\right\}_{1}^{\infty}$ satisfies $\underline{B} x^{\circ}(t+1)=A x^{\circ}(t), t \geqq 1$, then the sequence $\{z(t)\}_{1}^{\infty}$ defined by $(9.6 .10)$ satisfies:

$$
z_{i}(t)=0, t \geqq i, \text { if } \lambda_{i i}=0
$$

b) If a sequence of $k$-dimensional real vectors $\left\{u^{0}(t)\right\}_{1}^{\infty}$ satisfies $\underline{B}^{\prime} u^{\circ}(t)=A^{\prime} u^{\circ}(t+1), t \geq 1$, then the sequence $\{w(t)\}_{1}^{\infty}$ defined by $(9.6 .11)$ satisfies:

(*) This wel1-known property can easily be proved by combining the theorems 2.2 .8 and 2.2 .1 of Ortega and Rheinboldt (7). 


$$
w_{i}(t)=0, t \geqq 1, \text { if } \lambda_{i i}=1 \text {. }
$$

c) If $\left\{x^{\circ}(t)\right\}_{1}^{\infty} \in x^{\circ}$, then

$$
x^{0}(t) \in x^{0}, t \geq 1
$$

d) If $\left\{u^{o}(t)\right\}_{1}^{\infty} \in \underline{U}^{0}$, then

$$
\left(\underline{B}^{\prime}-\pi \underline{A}^{\prime}\right) \mathbf{u}^{\circ}(t) \in \mathbf{u}^{\circ}, t \geq 1
$$

e) If $\bar{x} \in x^{\circ}$, then one and only one sequence $\left\{x^{\circ}(t)\right\}_{1}^{\infty} \in x^{\circ}$ exists such that

$$
x^{\circ}(1)=\bar{x}
$$

f) If $\bar{u}$ satisfies ( $\left.\underline{B}^{\prime}-\pi A^{\prime}\right) \bar{U} \in U^{\circ}$, then one and only one sequence $\left\{\mathrm{u}^{\circ}(t)\right\}_{1}^{\infty} \in{\underline{U^{\circ}}}^{\circ}$ exists such that

$$
u^{o}(t)=\bar{u} \text {. }
$$

g) For every $\varepsilon>0$ a period $T_{\varepsilon} \geqq 1$ exists such that every $\left(\{x(t)\}_{1}^{\infty},\{y(t)\}_{1}^{\infty}\right) \in \underline{X Y}$ satisfies:

$$
\begin{gathered}
\left\|\left(\frac{1}{\rho}\right)^{t+T}(x(t+T), y(t+T))-(\hat{x}, \hat{y})\right\|_{\infty} \leqq E\left\|\left(\frac{1}{\rho}\right)(x(t), y(t))-(\tilde{x}, \hat{y})\right\|_{\infty}, \\
t \geq 1, T \geqq T E
\end{gathered}
$$

h) For every $\varepsilon>0$ a period $T_{\varepsilon} \geqq 1$ exists such that every $\left(\{\mathrm{u}(\mathrm{t})\}_{1}^{\infty},\{\mathrm{v}(\mathrm{t})\}_{1}^{\infty}\right\} \in \hat{\mathrm{U} V}$ satisfies:

$$
\begin{gathered}
\left\|\left(\frac{1}{\pi}\right)^{t+T}(u(t+T), v(t+T))-(\hat{u}, \tilde{v})\right\|_{\infty} \leq \varepsilon\left\|\left(\frac{1}{\pi}\right)^{t}(u(t), v(t))-(\tilde{u}, \tilde{v})\right\|_{\infty} \\
t \geqq 1, T \geqq T_{E} .
\end{gathered}
$$

i) A positive number $\delta_{1}$ exists such that every $\left((x(t)\}_{1}^{\infty},\{y(t)\}_{1}^{\infty}\right) \in \underline{X Y Y}$ satisfying 


$$
\left\|\left(\frac{1}{\rho}\right) T_{x}(T)-\tilde{x}\right\|_{\infty} \leq \delta_{1} \text {, for some } T \geqq 1 \text {, }
$$

also satisfies:

$$
x(t), y(t) \geqq 0 ; t \geqq T+1
$$

j) A positive number $\delta_{2}$ exists such that every $\left(\{u(t)\}_{1}^{\infty},\{v(t)\}_{1}^{\infty}\right) \in \underline{U V}$ satisfying

$$
\left\|\left(\frac{1}{\pi}\right)^{T} \mathrm{u}(T)-\tilde{u}\right\|_{\infty} \leq \delta_{2} \text {, for some } T \geq 1 \text {, }
$$

also satisfies:

$$
u(t), v(t) \geqq 0, t \geqq T \text {. }
$$

\section{Proof.}

(a) If $\left\{x^{\circ}(t)\right\}_{1}^{\infty}$ satisfies $\underline{B} x(t+1)=\underline{A x}(t), t \geqq 1$, then the sequence $\{z(t)\}_{1}^{\infty}$ defined by $(9.6 .10)$ satisfies $(9.6 .8)$. By virtue of proposition 9.7-a, this implies the validity of $9.8-\mathrm{a}$.

(b) With the help of $9.7-b$, this property can be proved in a similar manner.

(c) If $\left\{x^{\circ}(t)\right\}_{1}^{\infty} \in \underline{x}^{\circ}$ then (viz. def. 9.6.19)

$$
\left(\frac{1}{\rho}\right)^{t} x^{0}(t) \rightarrow 0, t \rightarrow \infty .
$$

Since $Q$ is invertible, the corresponding sequence $\{z(t)\}_{1}^{\infty}$ defined by $x^{\circ}(t)=Q z(t), t \geqq 1$ satisfies:

$$
\left(\frac{1}{\rho}\right)^{t} z(t) \rightarrow 0, t+\infty \text {. }
$$

Since $\{z(t)\}_{1}^{\infty}$ satisfies $(9.6 .8)$, this implies (viz. proposition $9.7-c$ ) that $z(t) \in z, t \geqq 1$, and successively (viz. def. 9.6 .17$): x^{\circ}(t) \in x^{0}, t \geqq 1$.

(d) This property can be proved in a similar manner as $9.8-\mathrm{c}$. 
(e) Firstly, we observe that $Q S=\operatorname{conj}(Q)$ (viz.9.6.12) implies the equivalence of the following equalities:

$$
\begin{aligned}
& \operatorname{conj}(Q z)=Q z, \\
& S^{-1} z=\operatorname{conj}(z),
\end{aligned}
$$

for $Q z=Q S S^{-1} z=\operatorname{conj}(Q)\left(S^{-1} z\right)$ and $\operatorname{conj}(Q z)=\operatorname{conj}(Q) \operatorname{conj}(z)$; which implies the equivalence.

Secondly, we observe that $s^{-1} \Lambda s=\operatorname{conj}(\Lambda)$ implies:

$$
s^{-1} \AA s=\operatorname{con} j(\Lambda) \text {, }
$$

$\bigwedge$ being the matrix defined by $(9.7 .7)$. For $s^{-1} \Lambda s=\operatorname{conj}(\Lambda)$ and definition $(9.7 .6)$ imply: $\left(S^{-1} \Lambda S\right)=\operatorname{conj}(\Lambda)$, so that: $I=\left(S^{-1} \underline{\Lambda}^{-1} \mathrm{~S}\right)\left(\mathrm{S}^{-1} \underline{\Lambda} \mathrm{S}\right)=\left(\mathrm{S}^{-1} \underline{\Lambda}^{-1} \mathrm{~S}\right) \operatorname{con} \mathrm{j}(\underline{\Lambda})$. Since $\operatorname{conj}\left(\underline{\Lambda}^{-1}\right) \operatorname{conj}(\underline{\Lambda})=I$, the latter implies: $\left(S^{-1} \underline{\Lambda}_{S}\right)=\operatorname{conj}\left(\underline{\Lambda}^{-1}\right)$ and so (viz. def.9.7.7) the equality $(9.8 .3$ ), as well.

Now, let $\bar{x} \in x^{\circ}$, so that (viz. def. 9.6.17) there is a unique $\bar{z} \in Z, Q \bar{z}=\vec{x}$. This implies (proposition 9.7-e) the existence of a unique sequence $\{z(t)\}_{j}^{\infty} \in \underline{z}$, with $z(1)=\bar{z}$. Since this sequence also (proposition 9.7-c) satisfies $z(t) \in z, t \geqq 1$, and since the systems $(9.7 .5)$ and $(9.7 .8)$ are equivalent, we may conclude:

$$
z(t+1)=\Lambda z(t), t \geqq 1 .
$$

Since $\operatorname{Im}(Q \bar{z})=0$ implies (viz. 9.8.1 and 9.8.2) that $S^{-1} \bar{z}=$ $=\operatorname{conj}(\bar{z})$, we may conclude by deduction $(9.8 .3$ and 9.8 .4$)$ : $S^{-1} z(t)=\operatorname{conj}(z(t)), t \geqq 1$.

By virtue of the equivalence of $(9.8 .1)$ and $(9.8 .2)$, this implies: $\operatorname{conj}(Q z(t))=Q z(t), t \geqq 1$. Thus, we may conclude that $\left\{x^{o}(t)\right\}_{1}^{\infty}$, defined by 


$$
x^{\circ}(t):=Q z(t), t \geqq 1
$$

is a sequence of real k-dimensional vectors. The equalities $(9.6 .7),(9.6 .8)$, and $(9.6 .10)$ imply that $\left\{x^{\circ}(t)\right\}_{1}^{\infty}$ satisfies

$$
\underline{B} x(t+1)=\underline{A x}(t), t \geqq 1 \text {. }
$$

Moreover, since $\{z(t)\}_{1}^{\infty} \in \underline{Z}$ (viz, def. 9.6.15), this sequence converges in the following manner

$$
\left(\frac{1}{\rho}\right)^{t} x^{o}(t) \rightarrow 0, t \rightarrow \infty .
$$

From $(9.8 .5)$ and $(9.8 .6)$ we may conclude that $\left\{x^{\circ}(t)\right\}_{1}^{\infty} \in \underline{x}^{\circ}$. Since $\bar{x}=Q z(1)$ and $(9.8 .5)$ implies $x^{0}(1)=\bar{x}$, and since $\{z(t)\}_{2}^{\infty}$ is unique, we may state that $9.8-e$ is proved.

(f) This property can be proved in a similar manner as $9.8-e$.

(g) and (i) For every $\left(\{x(t)\}_{1}^{\infty},\{y(t)\}_{1}^{\infty}\right) \in \underline{X}$ a sequence $\{z(t)\}_{1}^{\infty} \in \underline{z}$ exists such that

$$
\left(x(t)-\rho^{t} x\right)=\operatorname{spq} z(t), t \geqq 1
$$

With the help of $9.7-g$, we may conclude that numbers $\tilde{\rho} \in\left[0, \rho\left[\right.\right.$ and $N_{]}$exist. such that

$$
\left\|x(t+T)-\rho^{t+T} x\right\|_{\infty} \leq \rho^{t}\|S p Q\|_{\infty} N_{1}\left\|Q^{-1} s \dot{p}\right\|_{\infty}\left\|x(T)-\rho^{T}\right\|_{\infty}, t \geq 0, T \geqq 1,
$$

for all $\left(\{x(t)\}_{1}^{\infty},\{y(t)\}_{1}^{\infty}\right) \in \underline{X Y}$. We write $(9.8 .7)$ in the form:

$$
\left\|\left(\frac{1}{\rho}\right)^{t+T} \times(t+1)-\tilde{x}^{\eta}\right\|_{\infty} \leq\left(\frac{\tilde{\rho}}{\rho}\right){ }^{t} M_{1}\left\|\left(\frac{1}{\rho}\right) T x(T)-\tilde{x}_{\infty}\right\|_{\infty}, t \geqq 0, T \geqq 1,
$$

where $M_{1}=\|S p Q\|_{\infty} N_{1}\left\|Q^{-1} \operatorname{sip}\right\|_{\infty}$.

Since every $\left(\{x(t)\}_{1}^{\infty},\{y(t)\}_{1}^{\infty}\right) \in \underline{X_{Y}^{N}}$ satisfies 


$$
B\left(\left(\frac{1}{\rho}\right)^{t+1} x(t+1)-\tilde{x}\right)-\frac{1}{\rho} A\left(\left(\frac{1}{\rho}\right)^{t} x(t)-\tilde{x}\right)+\left(\left(\frac{1}{\rho}\right)^{t+1} y(t+1)-\tilde{y}\right) \leqq 0, t \geq 0,
$$

we may conclude:

$$
\begin{aligned}
\left\|\left(\frac{1}{\rho}\right)^{t+1} y(t)-\tilde{y}\right\|_{\infty} & \leqq\|B\|_{\infty}\left\|\left(\frac{1}{\rho}\right)^{t+1} x(t+1)-\tilde{x}\right\|_{\infty}+ \\
& +\frac{1}{\rho}\|A\|_{\infty}\left\|\left(\frac{1}{\rho}\right)^{t} x(t)-\tilde{x}\right\|_{\infty}, t \geq 0,
\end{aligned}
$$

and successively:

$$
\begin{aligned}
\left\|\left(\frac{1}{\rho}\right)^{t+1}(x(t+1), y(t+1))-(\tilde{x}, \tilde{y})\right\|_{\infty} \leqq\left(1+\|B\|_{\infty}\right)\left\|\left(\frac{1}{\rho}\right)^{t} x(t+1)-\tilde{x}\right\|_{\infty}+ \\
+\frac{1}{\rho}\|A\|_{\infty}\left\|\left(\frac{1}{\rho}\right)^{t} x(t)-\tilde{x}\right\|_{\infty}, t \geqq 0, \quad(9.8 .9)
\end{aligned}
$$

Combining (9.8.8) and (9.8.9), we may canclude that, for $M_{2}:=\left(1+\|B\|_{\infty}+\frac{1}{\rho}\|A\|_{\infty}\right) M_{1}$, all $\left(\{x(t)\}_{1}^{\infty},\{y(t)\}_{1}^{\infty}\right) \in \underline{X Y}$ satisfy:

$$
\left\|\left(\frac{1}{\rho}\right)^{t+T}(x(t+T), y(t+T))-(\tilde{x}, \tilde{y})\right\|_{\infty} \leqq\left(\frac{\tilde{\rho}}{\rho}\right)^{t} M_{2}\left\|\left(\frac{1}{\rho}\right)^{T} x(T)-\tilde{x}\right\|_{\infty},
$$

$$
t \geq 1, T \geqq 1, \quad(9.8 .10)
$$

where $(\stackrel{\tilde{\rho}}{\rho}) \in[0,11$.

Since $\left(\frac{\tilde{g}}{\rho}\right) \in\left[0,1\left[\right.\right.$, for every $\varepsilon>0$ a period $T_{\varepsilon}$ exists such that

$$
\left(\frac{\tilde{\rho}}{\rho}\right)^{T} M_{2} \leqq \varepsilon, T \geqq T_{\varepsilon}
$$

From $(9.8 .10)$ and $(9.8 .11)$ we may conclude

$$
\begin{aligned}
\|\left(\frac{1}{\rho}\right)^{t+T}\left(x(t+T), y(t+T)-(\hat{x}, \tilde{y}) \|_{\infty} \leqq\right. & \varepsilon\left\|\left(\frac{1}{\rho}\right)^{t}(x(t), y(t))-(\tilde{x}, \tilde{y})\right\|_{\infty}, \\
t & \geqq 1, T \geqq T \varepsilon_{\varepsilon} .
\end{aligned}
$$

This proves property 9.8-g. Property 9.8-i can be proved by defining the positive number

$$
\delta_{1}:=\frac{1}{M_{2}} \min \left(\tilde{x}_{j}, \tilde{y}_{i}\right) \mid \tilde{x}_{j}>0, \tilde{y}_{i}>0,
$$


so that we may conclude from (9.8.10) and from the definition $(9.6 .21)$ of $\underline{X Y}$, that

$$
\left\|\left(\frac{1}{\rho}\right)^{T} x(T)-\tilde{x}\right\|_{\infty} \leqq \delta
$$

implies:

$$
\begin{aligned}
\|\left(\frac{1}{\rho}\right)^{t+T}(x(t+T) & , y(t+T))-(\tilde{x}, \tilde{y}) \|_{\infty} \leqq \\
& \leqq\left[\min \left(\tilde{x}_{j}, \tilde{y}_{i}\right) \mid \widetilde{x}_{j}>0, \tilde{y}_{i}\right], t \geqq 1 .
\end{aligned}
$$

This implies the validity of property 9.8-i.

(h) and (j) These properties may be proved in a similar manner as $9.8-g, i$.

\subsection{Theorem.}

For every pair of semi equilibrium paths $((x, y),(u, v))$ and $((x, y),(q, y))$, belonging to a non-degenerated equilibrium combination, the following properties hold:

a) If, for some $T \geqq 1$ :

$$
(x(T), y(T))=(x(T), y(T)),
$$

then:

$$
(x(t), y(t))=(x(T), y(T)), t \geqq T .
$$

b) If, for some $T \geqq 1$ :

$$
(x(T), v(T))=(\underset{v}{u}(T), \underset{v}{v}(T)),
$$

then:

$$
(u(t), v(t))=(\underline{u}(t), \chi(t)), t \geqq T .
$$




\section{Proof.}

Let $(x, y)$ and $(x, y)$ be the primal parts of semi equilibrium paths belonging to a non-degenerated equilibrium combination $(\tilde{x}, \tilde{y}),(\tilde{u}, v)$. When we define:

$$
\left.x^{o}(t):=\operatorname{sip}^{\prime}(x)-x(t)\right), t \geqq 1
$$

then it follows from proposition $9.4-b$ that

$$
\underline{B} x^{0}(t+1)=A x^{0}(t), t \geq 1
$$

This implies, by virtue of proposition $9.8-a$, the existence of a sequence $\{z(t)\}_{1}^{\infty}$ satisfying

$$
\begin{array}{ll}
x^{0}(t)=Q z(t), & t \geqq 1, \\
\Lambda z(t+1)=\frac{1}{\pi}(\Lambda-I) z(t), & t \geqq 1, \\
z_{i}(t)=0, & t \geqq 1, \text { if } \lambda_{i i}=0 .
\end{array}
$$

Now suppose that $(x(T), y(T))=(X(T), Z(T))$ for some $T \geqq 1$. Then, $(9.9 .5)$ and $(9.9 .6)$ imply $z(T)=0$, and so, by virtue of $(9.9 .7):$

$$
z_{i}(t)=0, t \geqq T \text {, if } \lambda_{i i} \neq 0
$$

Clearly, $(9.9 .8)$ and $(9.9 .9)$ imply: $z(t)=0, t \geqq T$, and so, in connection with $(9.9 .6)$ and $(9.9 .5)$, we may conclude: $(x(t), y(t))=(x(t), y(t)), t \geqq T$.

The b) part of the theorem can be proved in a similar manner.

\section{9,10 Remark.}

In theorem 9.2 we have found that all consistent semi equilibrium paths $((x, y),(u, v))$ of an LP-problem(P- or D-directed; exponential; virtually superregular) satisfy: 


$$
\begin{aligned}
& \left.\left(x_{1 / \rho}, y_{1 / \rho}\right) \in 1_{\infty}^{n_{x}}\right|_{\infty} ^{m} \\
& \left.\left(u_{1 / \pi}, v_{1 / \pi}\right) \in 1_{\infty}^{m_{x 1}}\right|_{\infty} ^{n} .
\end{aligned}
$$

Using the definitions $(9.6 .8)$ to $(9.6 .11)$, one can dexive that, for every consistent semi equilibrium path $((x, y),(u, v))$ belonging to a non-degenerated equilibrium combination $(\tilde{x}, \tilde{y}),(\tilde{u}, \tilde{v})$, sequences of complex vectors $\{z(t)\}_{1}^{\infty}$ and $\{w(t)\}_{1}^{\infty}$ exist such that

$$
\begin{array}{cc}
x(t)=\operatorname{spQz}(t), & t \geqq 1, \\
\Lambda z(t+1)=\frac{1}{\pi}(\Lambda-I) z(t), & t \geqq 1, \\
\left(\underline{B}^{\prime}-\pi A^{\prime}\right) S_{d} u(t)=\left(Q^{-1}\right) w(t), & t \geqq 1, \\
\Lambda w(t)=\frac{1}{\pi}(\Lambda-I) w(t+1), & t \geqq 0 .
\end{array}
$$

Moreover, proposition $9.8-a, b$ implies:

$$
\begin{aligned}
& z_{i}(t)=0, t \geq 1, \text { if } \lambda_{i i}=0, \\
& w_{i}(t)=0, t \geq 1, \text { if } \lambda_{i i}=1 .
\end{aligned}
$$

Since $(9.10 .1),(9.10 .2)$, and $(9.10 .4)$ imply

$$
\begin{aligned}
& \left\|\left(\frac{1}{\rho}\right)^{t} z(t)\right\|_{\infty} \leqq M_{1}, t \geqq 1, \text { for some } M_{1}, \\
& \left\|\left(\frac{1}{\pi}\right)^{t} u(t)\right\|_{\infty} \leqq M_{2}, t \geqq 1, \text { for some } M_{2},
\end{aligned}
$$

one can derive from $(9.10 .3),(9.10 .6)$, and from $(9.10 .5),(9.10 .7)$ :

$$
\begin{aligned}
& z_{i}(t)=0, t \geqq 1, \text { if } \lambda_{i i}=0 \text { or }\left|\left(\lambda_{i i}-1\right) / \lambda_{i i}\right|>\rho \pi,(9.10 .8) \\
& w_{i}(t)=0, t \geqq 1, \text { if } \lambda_{i i}=1 \text { or }\left|\lambda_{i i} /\left(\lambda_{i i}-1\right)\right|>1,(9.10 .9)
\end{aligned}
$$

Thus we may conclude that for every consistent semi equilibrium path belonging to a non-degenerated equilibrium combination $(\tilde{x}, \tilde{y}),(\tilde{u}, \tilde{v})(\tilde{x}$ possessing at least one positive component), sequences of complex vectors $\{z(t)\}_{1}^{\infty}$ and $\{w(t)\}_{1}^{\infty}$ exist, satisfying $(9.10 .2),(9.10 .3),(9.10 .8)$ and $(9.10 .4),(9.10 .5),(9.10 .9)$. 
This means that, if there are no eigenvalues $\lambda_{i i}$ for which $\left|\left(\lambda_{i}-1\right) / \lambda_{i i}\right|$ is equal to $\rho \pi$ or to one, then such sequences $\{z(t)\}_{1}^{\infty}$ and $\{w(t)\}_{1}^{\infty}$ (viz. proposition $9.7-e, f$, and definitions 9.6 .13 to 9.6 .16$)$ satisfy

$$
\begin{aligned}
& \left(\frac{1}{p}\right)^{t} z(t) \rightarrow 0, t \rightarrow \infty, \\
& \left(\frac{1}{\pi}\right)^{t} w(t) \rightarrow 0, t \rightarrow \infty,
\end{aligned}
$$

which implies:

$$
\begin{aligned}
& \left(\frac{1}{\rho}\right)^{t}(x(t), y(t)) \rightarrow(\tilde{x}, \tilde{y}), t \rightarrow \infty, \\
& \left(\frac{1}{\pi}\right)^{t}(u(t), v(t)) \rightarrow(\tilde{u}, \tilde{v}), t \rightarrow \infty .
\end{aligned}
$$

Hence we may conclude: if $H:=\left(\underline{B}^{\prime}-\underline{A}^{\prime}\right)^{-1} \underline{B}$ has no eigenvalues $\lambda_{i i}$ for which $\left|\left(\lambda_{i i}-1\right) / \lambda_{i i}\right|$ is equal to $\rho \pi$ or to one, then all consistent semi equilibrium paths converge in the sense of $(9.10 .10)$.

\subsection{Definition.}

In the next theorem an LP-problem(P- or D-directed; $f(t)=\tilde{f}$, $p(t)=\tilde{p}, t \geqq k$; superregular) will be considered. A corresponding LP-problem(f(t)= $f, p(t)=\stackrel{\sim}{p}, t \geqq 1$ ) will be called the associated exponential LP-problem.

We observe that, if the original problem is P- or D-directed and virtually supperregular, then an associated exponential LP-problem is $P$ - or $D$-directed and virtually supperregular, as well.

A combination of vectors $(\tilde{x}, \tilde{y}),(\tilde{u}, \tilde{v})$ is called an equilibrium combination of an LP-problem( $f(t)=\tilde{f}, p(t)=\tilde{p}, t \geqq k)$ if it is an equilibrium combination of the associated exponential LP-problem. 


\subsection{Theorem.}

If $(\tilde{x}, \tilde{y}),(\tilde{u}, \tilde{v})$ is an equilibrium combination of an LP-problem $(P$ - or D-directed; $f(t)=\tilde{F}, P(t)=\tilde{P}, t \geqq K$; superregular), which satisfies the following conditions:

a) $(\tilde{x}, \tilde{y}),(\tilde{u}, \tilde{v})$ is non-degenerated,

b) Every consistent semi equilibrium path $((x, y),(u, v))$ of the associated exponential LP-problem, belonging to this equilibrium combination converges in the following manner:

$$
\left.\begin{array}{ll}
\left(\frac{1}{\rho}\right)^{t}(x(t), y(t))+(\tilde{x}, \tilde{y}), & t \rightarrow \infty \\
\left(\frac{1}{\pi}\right)^{t}(u(t), v(t)) \rightarrow(\tilde{u}, \tilde{v}), & t \rightarrow \infty
\end{array}\right\}, \quad(9 \cdot 12 \cdot 1)
$$

c) The associated exponential LP-problem is:regular; far the initial vector $x(0):=\tilde{x}$,

then the following properties hold:

d) A period $T$ exists such that for all optimal solutions $((\hat{x}, \hat{y}),(\hat{u}, \hat{v}))$ of the original LP-problem:

$$
\begin{array}{ll}
(\tilde{v}, \tilde{u})^{\prime}(\hat{x}(t), \hat{y}(t))=0, & t \geqq T, \quad(9 \cdot 12 \cdot 2) \\
(\tilde{y}, \tilde{x})^{\prime}(\hat{u}(t), \hat{v}(t))=0, & t \geqq T, \quad(9 \cdot 12 \cdot 3)
\end{array}
$$

e) All optimal solutions $((\hat{x}, \hat{y}),(\hat{u}, \hat{v}))$ of the original problem converge in the following manner:

$$
\begin{aligned}
& \left(\frac{1}{\rho}\right)^{t}(\hat{x}(t), \hat{y}(t))+(\tilde{x}, \tilde{y}), \\
& \left(\frac{1}{\pi}\right)^{t}(\hat{u}(t), \hat{v}(t)) \rightarrow(\tilde{u}, \tilde{v}), \\
&
\end{aligned}
$$

\section{Proof.}

Let $(\tilde{x}, \tilde{y}),(\tilde{u}, \tilde{v})$ be an equilibrium combination which satisfies the conditions $a, b$, and $c$. 
Define the sets $F^{\circ} \subset 1_{\infty}^{m}$ and $P \subset 1_{\infty}^{n}$ as follows:

$\left.\begin{array}{l}F^{\circ}:=\left\{g^{0} \mid g^{0}:=\lambda f^{\circ}+(1-\lambda)(\hat{f}+\hat{A} \tilde{x}, \ldots, \ldots, \hat{f}, \ldots)^{\prime}, \lambda \in[0,1]\right\} \\ p \quad:=\left\{q \mid q \quad:=\lambda p+(1-\lambda)(\hat{p}, \tilde{p}, \ldots, \hat{p}, \ldots,)^{\prime}, \lambda \in[0,1]\right\}\end{array}\right\}$

$(9.12 .6)$

This definition implies that $F^{\circ}$ and $\mathrm{p}$ are closed and bounded sets in 1-dimensional subspaces of $1_{\infty}^{\mathrm{m}}$ and $1_{\infty}^{\mathrm{n}}$. This implies the following property:

1) $F^{\circ}$ and $P$ are compact.

Now we consider, for each vector $\left(g^{\circ}, q\right) \in F^{\circ} \times P$ the following LP-problem,

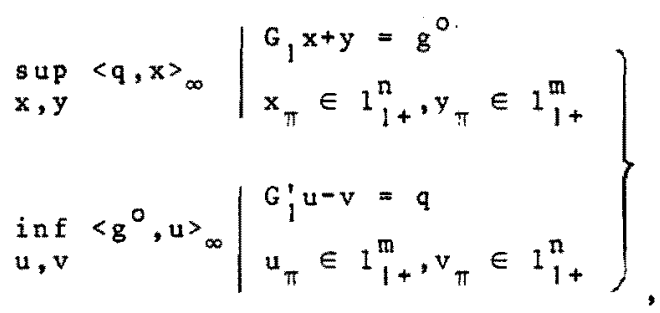

where $G$ is the matrix (def. 6.2.1) of the original problem. For every $\left(\mathrm{g}^{0}, q\right) \in \mathrm{F}^{0} \times p$, the set of $1 / 0$-transtorms of primal optimal solutions is denoted by $\hat{X Y}\left(g^{\circ}, q\right)$, and the set of $1 / \pi-$ transforms of dual optimal solutions by $\hat{U V}\left(g^{\circ}, q\right)$.

Since the original problem is $\mathrm{P}$ - or $\mathrm{p}$-directed and superregular, and since the associated exponential LP-problem is regular, the following properties hold:

2) For every $\left(g^{0}, q\right) \in F^{0} \times P$ : the LP-problem $(9.12 .7)$ is $P$ - or D-directed and strong regular.

3) For every $\left(g^{\circ}, q\right) \in F^{\circ} \times P$ (property 1, property 2, and theorem 7.3 )

$$
\begin{aligned}
& \hat{X Y}\left(g^{0}, q\right) \neq \emptyset \\
& \hat{U V}\left(g^{0}, q\right) \neq
\end{aligned}
$$


4) Numbers $M_{1}$ and $M_{2}$ exist (property 1, property 2, and theorem 7.3) such that:

$$
\begin{aligned}
& \hat{X} \hat{Y}\left(F^{0} \times P\right) \subset \sigma_{\infty}^{n}\left(M_{1}\right) \times \sigma_{\infty}^{m}\left(M_{1}\right) \\
& \hat{U} \hat{V}\left(F^{0} \times P\right) \subset \sigma_{\infty}^{m}\left(M_{2}\right) \times \sigma_{\infty}^{n}\left(M_{2}\right)
\end{aligned}
$$

5) For every $\left(g^{\circ}, q\right) \in F^{\circ} \times p$ (property 2 and theorem 6.19):

$$
\langle v, x\rangle_{\infty}+\langle u, y\rangle_{\infty}=0,(x, y) \in \hat{X} \hat{Y}\left(g^{\circ}, q\right),(u, v) \in \hat{U} \hat{V}\left(g^{0}, q\right) .
$$

6) For every $\left(g^{\circ}, q\right) \in F^{\circ} \times p$ and every number $\alpha>0$, neighbourhoods $\Omega\left(g^{0}, q ; \alpha\right) \subset 1_{\infty}^{m} \times 1_{\infty}^{n}$ of $\left(g^{\circ}, q\right)$ exist (property 1 , property 2 , and theorem 7.8 ) such that:

$\hat{X Y}\left(g^{0}, q\right) \oplus\left(\operatorname{int}\left(\sigma_{\infty}^{\mathrm{n}}(\alpha)\right) \times \operatorname{int}\left(\sigma_{\infty}^{\mathrm{m}}(\alpha)\right)\right) \supset \hat{\mathrm{XY}}\left(\Omega\left(\mathrm{g}^{0}, q ; \alpha\right) \cap\left(\mathrm{F}^{0} \times \mathrm{P}\right)\right)$ $\hat{U V}\left(g^{0}, q\right) \oplus\left(\operatorname{int}\left(\sigma_{\infty}^{m}(\alpha)\right) \times \operatorname{int}\left(\sigma_{\infty}^{n}(\alpha)\right)\right) \supset \hat{U V}\left(\Omega\left(g^{0}, q ; \alpha\right) \cap\left(F^{0} \times P\right)\right)$

7) If $((x, y),(u, v)) \in \hat{X Y}\left(F^{\circ} \times P\right) \times \hat{v V}\left(F^{\circ} \times P\right)$ satisfies

$$
\left.\begin{array}{l}
(\hat{u}, \tilde{v}) \prime(x(t), y(t))=0 \\
(\hat{y}, \tilde{x}) \cdot(u(t), v(t))=0
\end{array}\right\} \quad t \geqq L \quad \text { for some } L \geq K, \quad(9.12 .8)
$$

(K being the period for which: $f(t)=t, p(t)=\stackrel{p}{p}, t \geqq k$ ), then:

$$
\begin{aligned}
& (x(t), y(t)) \rightarrow(\tilde{x}, \tilde{y}), t+\infty, \\
& (u(t), v(t)) \rightarrow(\tilde{u}, \tilde{v}), t \rightarrow \infty,
\end{aligned}
$$

For

$$
\left(\left(\left\{\rho^{t} x(t-L)\right\}_{t=1}^{\infty},\left\{\rho^{t} y(t-L)\right\}_{t=1}^{\infty}\right),\left(\left\{\pi^{t} u(t-L)\right\}_{t=1}^{\infty},\left\{\pi^{t} v(t-L)\right\}_{t=1}^{\infty}\right)\right)
$$

is a semi equilibrium path of the associated exponential LPproblem with the initial vector $x(0) .:=x(L)$. Hence, condition b) implies this property. 
8) For every $\varepsilon>0$ a period $T_{\varepsilon}$ exists, such that all vectors $((\mathrm{x}, \mathrm{y}),(\mathrm{u}, \mathrm{v})) \in \hat{X Y}\left(\mathrm{~F}^{\circ} \times \mathrm{P}\right) \times \hat{U V}\left(\mathrm{~F}^{\circ} \times \mathrm{P}\right)$ which satisfy $(9.12 .8)$, also (proposition $9,8-g, h$ and property 7 ) satisfy:

$$
\left.\begin{array}{l}
\left\|\left(x\left(t+T_{\varepsilon}\right), y\left(t+T_{\varepsilon}\right)\right)-(\tilde{x}, \tilde{y})\right\|_{\infty} \leqq \varepsilon\|(x(t), y(t))-(\tilde{x}, \tilde{y})\|_{\infty} \\
\left\|\left(u\left(t+T_{\varepsilon}\right), v\left(t+T_{E}\right)\right)-(\tilde{u}, \tilde{v})\right\|_{\infty} \leqq \varepsilon\|(u(t), v(t))-(\hat{u}, \tilde{v})\| \infty
\end{array}\right\} \geqq L \text {. }
$$

$9)$ For every $\alpha>0$ a period $T$ exists, such that all vectors $((x, y),(u, v)) \in \hat{X Y}\left(E^{0} \times P\right) \times \hat{U V}\left(F^{\circ} \times P\right)$ which satisfy $(9.12 .8)$, also satisfy:

$$
\left.\begin{array}{l}
\|(x(t), y(t))-(\tilde{x}, \tilde{y})\|_{\infty}<a \\
\|(u(t), v(t))-(\tilde{u}, \tilde{v})\|_{\infty}<\alpha
\end{array}\right\} \quad t \geq L+T \alpha .
$$

For property 4) implies:

$$
\left.\begin{array}{l}
\|(x(t), y(t))-(\tilde{x}, \tilde{y})\|_{\infty} \leqq M_{1}+\|(\tilde{x}, \tilde{y})\|_{\infty} \\
\|(u(t), v(t))-(\tilde{u}, \tilde{v})\|_{\infty} \leqq M_{2}+\|(\tilde{u}, \tilde{v})\|_{\infty}
\end{array}\right\} \geqq 1,
$$

and so, when we choose the positive number $\varepsilon$ of property 8 ) in such a maner that

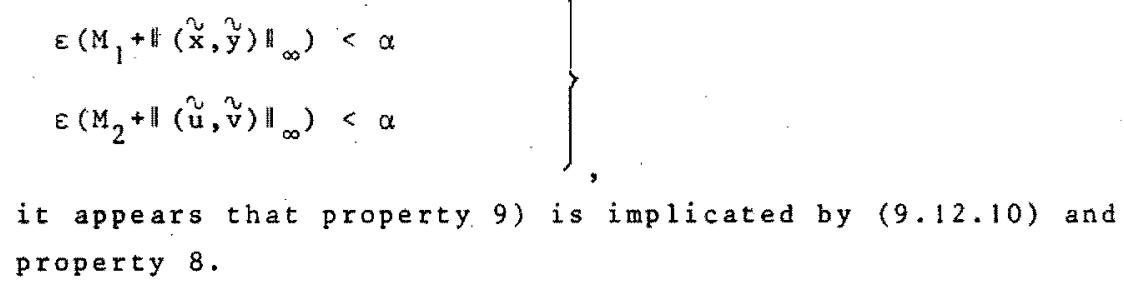

Now we choose the positive number $\alpha$ of property 6 and property 9 , as follows:

$\alpha:=\frac{1}{4} \min \left[\min (\hat{x}, \tilde{y})_{j} \mid(\hat{x}, \hat{y})_{j}>0\right],\left[\min _{j}(\tilde{u}, \hat{v})_{j} \mid(\hat{u}, \tilde{v})_{j}>0\right]$. 
This definition implies the validity of the next property:

10) If, for some $\left(g^{0}, q\right) \in F^{0} \times P$, a vector $((x, y) ;(x, y)) \in \hat{X Y}\left(g^{0}, q\right) \times \hat{U V}\left(g^{0}, q\right)$ and a period $s \geqq K$ exist, such that

$$
\left.\begin{array}{l}
\|(x(t), y(t))-(\tilde{x}, \tilde{y})\|_{\infty} \leqq 3 \alpha \\
\|(u(t), v(t))-(\tilde{u}, \tilde{v})\|_{\infty} \leqq 3 \alpha
\end{array}\right\} \quad t \geqq s,
$$

then, all vectors $((x, y),(u, v)) \in \hat{X Y}\left(g^{o}, q\right) \times \hat{U} \hat{V}\left(g^{\circ}, q\right)$ satisfy:

$$
\left.\begin{array}{l}
(\tilde{v}, \tilde{u})^{\prime}(x(t), y(t))=0 \\
(\tilde{y}, \tilde{x}) !(u(t), v(t))=0
\end{array}\right\} \quad t \geqq S .
$$

For $(9.12 .11)$ and $(9.12 .12)$ imply:

$$
\left.\begin{array}{l}
(x(t), y(t)) \geqq 1(\tilde{x}, \tilde{y}) \\
(u(t), v(t)) \geqq 1(\tilde{u}, \tilde{v})
\end{array}\right\} \quad t \geqq s,
$$

and so, since $(\tilde{x}, \tilde{y}),(\tilde{u}, \tilde{v})$ is non-degenerated (def. 9.1), property 10 is implied by $(9.12 .14)$ and property 5 .

Now, consider the neighbourhoods $\Omega\left(\mathrm{g}^{\circ}, q ; \alpha\right)$ of proposition 6 , in which $\alpha$ is defined by $(9.12 .11)$. Since the sets $F^{\circ}$ and $P$ are compact (prop. 1), the following property holds (Heine-Bore1):

11) A finite number of vectors

$$
\begin{aligned}
& \left(g^{1}, q^{1}\right),\left(g^{2}, q^{2}\right), \ldots,\left(g^{k}, q^{k}\right) \in F^{\circ} \times P \text { exists such that: } \\
& \bigcup_{i=1}^{k} \Omega\left(g^{i}, q^{i} ; \alpha\right) \supset F^{\circ} \times P .
\end{aligned}
$$

Moreover, we may choose these vectors in such a manner (def. 9.12.6) that: 


$$
\begin{aligned}
& \left.\left(g^{\prime}, q^{\prime}\right)=\left((\hat{f}+\Delta x(0), \tilde{f}, \ldots, \hat{z}, \ldots)^{\prime}(\tilde{p}, \tilde{p}, \ldots, \tilde{p}, \ldots)^{\prime}\right)\right) \\
& \left(\mathrm{g}^{\mathrm{k}}, \mathrm{q}^{\mathrm{k}}\right)=\left(\mathrm{f}^{\mathrm{o}}, \mathrm{p}\right) \\
& \left.\Omega\left(g^{i+1}, q^{i+1} ; \alpha\right) \cap\left(g^{i}, q^{i} ; \alpha\right) \cap R^{\circ} \times p \neq \emptyset, i=1,2, \ldots, k-1\right)
\end{aligned}
$$

From $(9.12 .16)$ and from property 6 we may conclude:

12) For every pair $\left(g^{i}, q^{i}\right),\left(g^{i+1}, q^{i+1}\right)$, vectors

$$
\begin{aligned}
& \left(\left(x^{i}, y^{i}\right),\left(u^{i}, v^{i}\right)\right) \in \hat{X} \hat{Y}\left(g^{i}, q^{i}\right) \times \hat{U V}\left(g^{i}, q^{i}\right) \\
& \left(\left(x^{i+1}, y^{i+1}\right),\left(u^{i+1}, v^{i+1}\right) \in \hat{X} \hat{Y}\left(g^{i+1}, q^{i+1}\right) \times \hat{U V}\left(g^{i+1}, q^{i+1}\right)\right.
\end{aligned}
$$

exist, such that

$$
\left.\begin{array}{l}
\left\|x^{i+1}-x^{i}\right\|_{\infty}+\left\|y^{i+1}-y^{i}\right\|_{\infty}<2 \alpha \\
\left\|u^{i+1}-u^{i}\right\|_{\infty}+\left\|v^{i+1}-v^{i}\right\|_{\infty}<2 \alpha
\end{array}\right\} .
$$

13) If all vectors $((x, y),(u, v)) \in \hat{X Y}\left(g^{i}, q^{i}\right) \times \hat{U} \hat{V}\left(g^{i}, q^{i}\right), i<k$ satisfy:

$$
\left.\begin{array}{l}
(\tilde{v}, \tilde{u})^{\prime}(x(t), y(t))=0 \\
(\tilde{y}, \tilde{x})^{\prime}(u(t), v(t))=0
\end{array}\right\} \quad t \geqq L
$$

for some fixed period $L \geqq K$, then all vectors $((x, y),(u, v)) \in \hat{X Y}\left(g^{i+1}, q^{i+1}\right) \times \hat{U} \hat{v}\left(g^{i+1}, q^{i+1}\right)$ satisfy:

$$
\left.\begin{array}{l}
(\tilde{v}, \tilde{u})^{\prime}(x(t), y(t))=0 \\
(\tilde{y}, \tilde{x})^{\prime}(u(t), v(t))=0
\end{array}\right\} \quad t \geq L+T_{a}
$$

$\mathrm{T}_{\alpha}$ being the period of property 9 , which corresponds with the value of $\alpha$ as defined by $(9.12,10)$.

This property may be proved as follows. Since all vectors $((x, y),(u, v)) \in \hat{X Y}\left(g^{i}, q^{i}\right) \times \hat{U V}\left(g^{i}, q^{i}\right)$ satisfy $(9.12 .17)$, they 
also (property 9) satisfy (9.12.9). This implies (property

12) the existence of an

$((x, y),(u, v)) \in^{\circ} \hat{X Y}\left(g^{i+1}, q^{i+1}\right) \times \hat{U} \hat{V}\left(g^{i+1}, q^{i+1}\right)$ satisfying

$(9.12 .12)$ with $S:=L+T{ }_{\alpha}$, and thus (property 10) the

validity of property 13 .

With the help of the latter property, the proof of the theorem may be completed as follows.

\section{Since}

$\left(\left(\{x(t):=\tilde{x}\}_{1}^{\infty},\{y(t):=\hat{y}\}_{1}^{\infty}\right),\left(\{u(t):=\hat{u}\}_{1}^{\infty},\{v(t):=\hat{v}\}_{1}^{\infty}\right)\right) \in \hat{X Y}\left(g^{1}, q^{1}\right) \times \hat{u v v}\left(g^{1}, q^{l}\right)$

$(9.12 .16$ and $\$ 8.1)$, a 11 vectors

$((x, y),(u, v)) \in \hat{X Y}\left(g^{1}, q^{\prime}\right) \times \hat{U V}\left(g^{\prime}, q^{1}\right)$ satisfy (property 10$)$ the equalities $(9.12 .17)$, with $L=K$. Applicating property 13 repeatedly, we find

$$
\left.\begin{array}{l}
(\tilde{v}, \tilde{u})^{\prime}(x(t), y(t))=0 \\
(\tilde{y}, \tilde{x})^{\prime}(u(t), v(t))=0
\end{array}\right\} \quad t \geq k+(i-1) T_{\alpha},
$$

for all vectors $((x, y),(u, v)) \in \hat{X} \hat{Y}\left(g^{i}, q^{i}\right) \times \hat{U V}\left(g^{i}, q^{i}\right)$, $i=1,2, \ldots, k$. Since $\left(\mathrm{g}^{k}, \mathrm{q}^{\mathrm{k}}\right)=\left(\mathrm{f}^{\circ}, \mathrm{p}\right)$, these inequalities prove the d) part of the theorem.

The e) part of the theorem is implied by d), property 7, and by the definition of the sets $\hat{X Y}(g, q)$ and $\hat{U V}(g, q)$.

\section{9,13 Theorem.}

An LP-problem(P- or D-directed; exponential; virtually superregular) possesses at most one equilibrium oombination $(x, \hat{y}),(\tilde{u}, \tilde{v})$ which satisfies simultaneously:

a) $(\tilde{x}, \tilde{y}),(\tilde{u}, \tilde{v})$ is non-degenerated.

b) Every semi equilibrium path $((x, y),(u, v))$ of $(\tilde{x}, \tilde{y}),(\tilde{u}, \tilde{v})$ converges in the following manner:

$$
\begin{aligned}
& \left(\frac{1}{\rho}\right)^{t}(x(t), y(t)) \rightarrow(\tilde{x}, \tilde{y}), t+\infty, \\
& \left(\frac{1}{\pi}\right)^{t}(u(t), v(t)) \rightarrow(\tilde{u}, \tilde{v}), t \rightarrow \infty,
\end{aligned}
$$


c) The LP-problem is regular for the initial vector $x(0):=\tilde{x}$.

\section{Proof.}

Suppose that $(\tilde{x}, \tilde{y}),(\tilde{u}, \tilde{y})$ and $(\bar{x}, \bar{y}),(\bar{u}, \bar{v})$ are equilibrium combinations, both satisfying $a, b$, and $c$. Then, by virtue of theorem 9.12, we may conclude that a period $s$ exists, such that every optimal solution $((x, y),(u, v))$, of the LP-problem with an initial vector $x(0):=\bar{x}$, satisfies:

$$
\left.\begin{array}{l}
(\tilde{v}, \tilde{u})^{\prime}(x(t), y(t))=0 \\
(\tilde{y}, \tilde{x}) \cdot(u(t), v(t))=0
\end{array}\right\} t \geq s .
$$

Since $\left(\left(\left\{\rho^{t} \bar{x}\right\}_{1}^{\infty},\left\{\rho^{t} \bar{y}\right\}_{1}^{\infty}\right),\left(\left\{\pi^{t} \bar{u}\right\}_{1}^{\infty},\left\{\pi^{t} \bar{v}_{1}^{\infty}\right)\right)\right.$ is an optimal solution of the LP-problem with $x(0):=\bar{x}$, it follows from $(9.13 .1)$ :

$$
\begin{aligned}
& (v, \tilde{u}) \cdot(\bar{x}, \bar{y})=0 \\
& \left(\begin{array}{c}
u \\
\bar{x}
\end{array}\right) \cdot(\bar{u}, \bar{v})=0
\end{aligned}
$$

Thus, it appears that $(\bar{x}, \bar{y}),(\tilde{u}, \tilde{v})$ and $(\tilde{x}, \tilde{y}),(\bar{u}, \bar{v})$ are both equilibrium combinations as well. Then, the definition of nondegenerated equilibrium paths ( $\$ 9.1)$ implies that $(\bar{x}, \bar{y})=(\bar{x}, \bar{y})$ and $(\bar{u}, \bar{v})=(\hat{u}, \tilde{v})$. 
10. EQUIVALENT LINEAR PROGRAMMING PROBLEMS OVER A FINITE HORIZON.

\subsection{Introduction.}

It appears that, if the conditions of theorem 9.12 are satisfied, a period $T$ exists such that all optimal solutions $((x, y),(u, v))$ of such an LP-problem satisfy:

$$
\left.\begin{array}{l}
(\tilde{v}, \tilde{u}) \cdot(x(t), y(t))=0 \\
(\tilde{y}, \tilde{x}) \cdot(u(t), v(t))=0
\end{array}\right\}, t \geq T .
$$

So, ever since period $T$ the optimal solutions may be interpreted as semi equilibrium paths belonging to the equilibrium combination $(\tilde{x}, \tilde{y}),(\tilde{u}, \tilde{v})$ as mentioned in theorem 9.12. Moreover, we found that the optimal solutions $((x, y),(u, v))$ satisfy:

$$
\left.\begin{array}{l}
\left(\frac{1}{\rho}\right)^{t}(x(t), y(t)) \rightarrow(\hat{x}, \tilde{y}), t \rightarrow \infty \\
\left(\frac{1}{\pi}\right)^{t}(u(t), v(t)) \rightarrow(\tilde{u}, \tilde{v}), t \rightarrow \infty
\end{array}\right\} .
$$

Now, let for this equilibrium combination $x^{\circ}, v^{\circ}, \underline{X Y}$ and $\underline{Y} Y^{\prime}$ be the sets defined by $(9.6 .17),(9.6 .18),(9.6 .21)$, and by $(9.6 .22)$. Then $(10.1 .1)$ and $(10.1 .2)$ imply that all optimal solutions $((x, y),(u, v))$ of this LP-problem satisfy:

$$
\left.\begin{array}{l}
\left(\{x(t)\}_{T}^{\infty},\{y(t)]_{T}^{\infty}\right) \in \underline{X Y} \\
\left.\left(\{u(t)\}_{T}^{\infty},\{v(t)\}_{T}^{\infty}\right) \in \tilde{U Y}\right\}
\end{array}\right\}
$$

Moreover, one can derive from 9.8-c, d that (10.13) implies:

$$
\left.\begin{array}{l}
s_{p}^{\prime}\left(x(t)-\rho^{t} x\right) \in x^{0} \\
\left(\underline{B}^{\prime}-\pi A^{\prime}\right) s_{d}\left(u(t)-\pi^{t} u\right) \in v^{0}
\end{array}\right\} t \geqq T,
$$

$S_{p}$ and $s_{d}$ being the matrices defined by $9.3-a, b$. 
It will appear that this offers the possibility to construct a linear programing problem over a finite horizon, from which all optimal solutions of the original infinite horizon problem can be found. To that end, we shall define a system of linear equalities generated by the sets $x^{\circ}$ and $U^{\circ}$.

\subsection{Definitions.}

For the equilibrium combination $(\tilde{x}, \hat{y}),(\tilde{u}, \tilde{v})$ as mentioned in theorem 9.12, we define the following matrices:

a) $C^{2}$ and $\mathrm{C}^{i}$, consisting of the real and the imaginary part resp. of the column vectors q,j of matrix $Q$ (def. 9.6.7), which correspond with eigenvalues $\lambda_{j j}$ of $\Lambda$ (def. 9.6.7) satisfying:

$$
\left.\begin{array}{l}
\lambda_{j j} \neq 0 \\
\left|\frac{\lambda_{j j}-1}{\lambda_{j j}}\right|<p \pi
\end{array}\right\}
$$

provided that such eigenvalues exist. (*)

b) $\mathrm{D}^{\mathrm{T}}$ and $\mathrm{D}^{i}$, consisting of the real and the imaginary part resp. of the column vectors q.j of matrix $Q$, which correspond with eigenvalues $\lambda_{j j}$ of $\Lambda^{\circ}$ satisfying:

$$
\begin{aligned}
& \lambda_{j j} \neq 0 \\
& \left|\frac{\lambda_{j j}-1}{\lambda_{j j}}\right| \leq 1
\end{aligned} \mid
$$

provided that such eigenvalues exist.

(*) In $\$ 10.6$ we shall revert to this subject. 
We observe that these definitions imply that the equalities

$$
\left.\begin{array}{l}
C^{x}=D^{x} \\
C^{i}=D^{i}
\end{array}\right\}
$$

are valid, if and only if, matrix $H:=(\underline{B}-\pi \underline{A})^{-1} \underline{B}$ (def. 9.6 .4 and $9.6 .7)$ contains no eigenvalues $\lambda_{j j}$ satisfying:

$$
p \pi \leqq\left|\frac{\lambda_{j j^{-1}}}{\lambda_{j j}}\right| \leqq 1
$$

\subsection{Proposition.}

For the sets $x^{\circ}$, $U^{\circ}$ (def. 9.6 .17 and 9.6 .18 ), the kxh, matrices $C^{r}, c^{i}$, and the $k \times h_{2}-m a t r i c e s ~ D^{r}, D^{i}$ defined by $10.2-a, b$, the following equalities hold:

$$
\begin{aligned}
& x^{\circ}=\left\{x^{\circ}:=C^{r} z^{r}-C^{i} z^{i} \mid C^{i} z^{r}+C^{r} z^{j}=0, z^{r}, z^{i} \in R_{1}^{h} \quad(10.3 .1)\right. \\
& U^{\circ}=\left\{w^{r} \mid \begin{array}{l}
D^{r^{\prime}} w^{r}+D^{i^{\prime}} w^{i}=0, D^{i} w^{r}-D^{r} w^{i}=0, \\
\text { for some } w^{i} \in R^{k}
\end{array} \quad(10.3 .2)\right.
\end{aligned}
$$

\section{Proof.}

The definitions $(9.6 .13)$ and $(9.6 .17)$ of the sets $z$ and $x^{\circ}$ imply:

$$
x^{0}=l^{x^{0} \in R^{k}} \mid \begin{aligned}
& x:=Q z, \text { for some z such that } \\
& \left.z_{i}=0 \text { if } \lambda_{i i}=0 \text { or }\left|\left(\lambda_{i i}-1\right) / \lambda_{i i}\right| \geqslant \rho \pi\right)
\end{aligned}
$$

Clearly, (10.3.3) and definition 10.2-a imply (10.3.1).

The definitions $(9.6 .14)$ and $(9.6 .18)$ of the sets $W$ and $U^{\circ}$ imply:

$$
v^{\circ}=\left\{\begin{array}{l}
u^{\circ} \in R^{k} \mid \begin{array}{l}
u^{\circ}:=\left(Q^{-1}\right) ' w, \text { for some w such that } \\
w_{i}=0 \text { if } \lambda_{i i}=1 \text { or }\left|\lambda_{i i}\left(\lambda_{i i}-1\right)\right| \geqslant 1
\end{array} .
\end{array}\right.
$$


Since $(10.3 .4)$ can be written:

$$
v^{\circ}=\left\{u^{\circ} \in R^{k} \mid \begin{array}{l}
Q^{\prime} u^{0}=w, \text { for some wuch that } \\
w_{i}=0 \text { if simultaneously } \lambda_{i i} \neq 0 \\
\text { and }\left|\left(\lambda_{i i}-1\right) / \lambda_{i i}\right| \leqq 1
\end{array}\right\}
$$

definition 10.2-b implies:

$$
U^{0}=\left\{u^{0} \in R^{k} \mid D^{r^{\prime}} u^{0}=0, D^{i^{\prime}} u^{o}=0\right\}
$$

From (9.6.12) and from definition $10.2-b$, one can derive that (10.3.5) may be written:

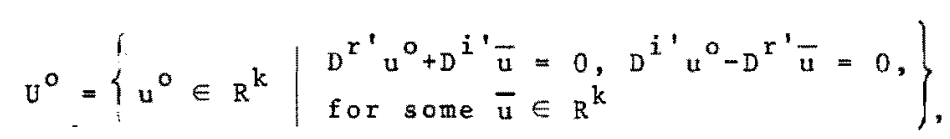

which is equivalent to $(10.3 .2)$.

\subsection{Definitions.}

Let $(\tilde{x}, \tilde{y}),(\hat{u}, \tilde{v})$ be an equilibrium combination of an Lp-problem as considered in theorem 9.12 , and let $\mathrm{C}^{\mathrm{r}}, \mathrm{C}^{\mathrm{i}}$ be the matrices defined by $10.2-a$, and $\hat{\delta}_{1}, \delta_{2}$ the positive numbers of proposition 9.8-i,j, then: for every period. $T>K$, we can associate an LPproblem over a finite horizon to the original infinite horizon problem, in the following manner:

the primal finite horizon problem:

$$
\begin{aligned}
& \phi\left(x, X, Y^{i}, Y^{2} ; T\right):=\left\langle p_{\pi}, x\right\rangle_{1, T}+\pi+{ }_{p}^{T+1} S_{p} X+\quad(10.4 .1) \\
& +\pi T+1\left(\hat{u}-\delta_{2} e\right)^{\prime} s_{d}^{\prime} Y^{1}-\pi^{T+1}\left(\hat{u}+\delta_{2} e\right)^{\prime} s_{d}^{\prime} Y^{2},
\end{aligned}
$$

to be maximalized over the vectors $\{(x(t), y(t))\}_{1}^{T}, X, Y^{l}, Y^{2}$, $x^{1}, x^{2}, z^{r}, z^{i}$, which satisfy: 


$$
\begin{aligned}
& B x(1)+y(1)=\rho f^{\circ}(1) \\
& B x(t+1)-A x(t)+y(t+1)=p^{t+1} f^{o}(t+1), t=1, \ldots T-1 \\
& (\underline{B}-\pi A) X-S A x(T)+Y^{1}-Y^{2}=\frac{p^{T+1}}{1-\rho \pi^{S}} d^{Y} \\
& x(T)+x^{1}=\rho^{T}(x+\delta, e) \\
& -x(T)+x^{2}=\rho^{T}\left(-\stackrel{n}{x}+\delta_{1} e\right) \\
& X+C^{r} z^{x}-C^{i} z^{i}=\frac{\rho^{T+1}}{1-\rho \pi} S_{p}^{x} \\
& \mathrm{C}^{i} z^{x}+\mathrm{C}^{x} z^{i}=0 \\
& x(t), y(t) \geq 0, t=1, \ldots, T \\
& x^{1}, x^{2}, y^{1}, y^{2} \geq 0
\end{aligned}
$$

and its dual problem, to be derived by application of the duality rules for linear programming in a finite dimensional space:

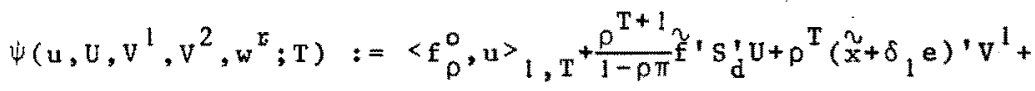

$$
+\rho^{T}(-x+\delta, e)^{\prime} \cdot v^{2}+\frac{\rho^{T+1} \eta}{1-\rho \pi} x^{3} s p^{r}
$$

to be minimalized over the vectors $\{(u(t), v(t))\}_{1}^{\mathrm{T}}, \mathrm{u}, \mathrm{v}^{l}, \mathrm{v}^{2}$, $\mathrm{U}^{1}, \mathrm{U}^{2}, \mathrm{w}^{\mathrm{T}}, \mathrm{w}^{i}$, which satisfy: 


$$
\begin{aligned}
& B^{\prime} u(t)-A^{\prime} u(t+1)-v(t)=\pi^{t} p(t), t=1, \ldots, T-1 \\
& B^{\prime} u(T)-A^{*} S_{d}^{\prime} U+V^{1}-V^{2}-v(T)=\pi T_{p}^{2} \\
& \mathrm{u}-\mathrm{u}^{l}=\pi^{\mathrm{T}+1} \mathrm{~S}_{\mathrm{d}}\left(\mathrm{u}-\delta_{2} \mathrm{e}\right) \\
& -v-u^{2}=\pi^{T+1} S_{d}\left(-\ddot{u}-\delta_{2} e\right) \\
& \left(\underline{B}^{\prime}-\pi \underline{A}^{\prime}\right) U+w^{r}=\pi^{T+1} S_{p}^{\prime} \hat{p} \\
& c^{x} w^{r}+c^{i} w^{i}=0 \\
& -c^{i} \cdot w^{x}+c^{r} \cdot w^{i}=0 \\
& u(t), v(t) \geqq 0, t=1, \ldots, T \\
& u^{1}, u^{2}, v^{1}, v^{2} \geq 0
\end{aligned}
$$

We shall denote the whole,consisting of the primal and the dual problem over a finite horizon, by $\overline{\mathrm{LP}}(\mathrm{T})$.

We sha11 call:

- $\{(x(t), y(t))\}_{1}^{T}$ a primal optimal solution of $\overline{L P}(T)$ if vectors $x, y^{1}, y^{2}, x^{1}, x^{2}, z^{r}$ and $z^{i}$ exist, such that $\{(x(t), y(t))\}_{1}^{T}$, $X, Y^{1}, Y^{2}, x^{1}, x^{2}, z^{r}, z^{j}$ satisfy $(10.4 .2)$ and such that, for these vectors, $\phi\left(X, X, Y^{\prime}, Y^{2} ; T\right)$ takes his maximum value.

$-\{(u(t), v(t))\}_{1}^{T}$ a dual optimal solution of $\overline{L P}(T)$ if vectors $u, v^{l}, v^{2}, u^{l}, u^{2}, w^{r}$ and $w^{i}$ exist, such that $\{(u(t), v(t))\}_{l}^{T}$, $u, v^{j},{ }^{2}, u^{1}, u^{2}, w^{r}, w^{i}$ satisfy $(10.4 .4)$ and such that, for these vectors, $\psi\left(u, U, v^{l}, v^{2}, w^{r} ; T\right)$ takes his minimal value.

$-\left(\{(x(t), y(t))\}_{1}^{T},\{(u(t), v(t))\}_{1}^{T}\right.$ an optimal solution of $\overline{L P}(T)$ if $\{(x(t), y(t))\}_{1}^{T}$ is primal optimal and $\{(u(t), v(t))\}_{1}^{T}$ is dual optimal.

Applying the duality properties of linear programing in a finite dimensional space, one can derive that solutions $\left(\{(x(t), y(t))\}_{1}^{T}, x, Y^{1}, y^{2}, x^{1}, x^{2}, z^{r}, z^{i}\right)$ and $\left(\{(u(t), v(t))\}_{1}^{T}, u, v^{1}, v^{2}, u^{1}, u^{2}, w^{r}, w^{i}\right)$ of $(10.4 .2)$ and $(10.4 .4)$ resp. are optimal if and only if: 


$$
\begin{aligned}
& (v(t), u(t))^{\prime}(x(t), y(t))=0, t=1, \ldots, T \\
& u^{\prime} \cdot y^{\prime}=0, u^{2} y^{2}=0 \\
& x^{\prime} \cdot v^{\prime}=0, x^{2} \cdot v^{2}=0
\end{aligned}
$$

We shall call an associated problem $\overline{\mathrm{LP}}(\mathrm{T})$ equivalent with respect to the original infinite horizon problem, if simultaneously the following relations between optimal solutions of $\overline{\mathrm{LP}}(\mathrm{T})$ and the original infinite horizon problem are valid:

a) If $((x, y),(u, v)):=\left(\left(\{x(t)\}_{1}^{\infty},\{y(t)\}_{1}^{\infty}\right),\left(\{u(t), v(t)\}_{1}^{\infty}\right)\right)$ is an optimal solution of the original infinite horizon problem, then $\left(\left\{(x(t), y(t)\}_{1}^{T},\left\{(u(t), v(t)\}_{l}^{T}\right)\right.\right.$ is an optimal solution of $\overline{\mathrm{LP}}(\mathrm{T})$.

b) If $\left(\{(x(t), y(t))\}_{1}^{T},\{(u(t), v(t))\}_{1}^{T}\right.$ is an optimal solution of $\overline{\mathrm{LP}}(\mathrm{T})$, then the original infinite horizon problem possesses an optimal solution $((\hat{x}, \hat{y}),(\hat{u}, \hat{v})):=\left(\left(\{\hat{x}(t)\}_{1}^{\infty},\{\hat{y}(t)\}_{l}^{\infty}\right),\left(\{\hat{u}(t)\}_{1}^{\infty},\{\hat{v}(t)\}_{1}^{\infty}\right)\right)$ such that

$$
\left.\begin{array}{l}
(\hat{x}(t), \hat{y}(t))=(x(t), y(t)) \\
(\hat{u}(t), \hat{v}(t))=(u(t), v(t))
\end{array}\right\} t=1, \ldots, T .
$$

\subsection{Theorem.}

If $(\tilde{x}, \tilde{y}),(u, \tilde{v})$ is an equilibrium combination of an LP-problem(Por D-directed; $f(t)=\tilde{f}, p(t)=\tilde{p}, t \geq k$; superregular), which possesses the following properties:

a) $(\tilde{x}, \tilde{y}),(\tilde{u}, \tilde{v})$ is non-degenerated, b) The matrix H (def. 9.6.4) possesses no eigenvalues $\lambda_{i i}$ for
which:

$$
\left|\frac{\lambda_{i i^{-}}}{\lambda_{i i}}\right| \in[\rho \pi, 1]
$$

c) The associated exponential LP-problem is regular for the initial vector $x(0):=\tilde{x}$, 
then, a period $\mathrm{T}^{*}>\mathrm{K}$ exist such that, for every $\mathrm{T} \geqq \mathrm{T}^{*}$, the associated problem over a finite horizon $\overrightarrow{\mathrm{LP}}(\mathrm{T})$ (provided the matrices $\mathrm{C}^{\mathrm{r}}$ and $\mathrm{C}^{i}$ exist) is equivalent with respect to the original problem over an infinite horizon.

\section{Proof.}

Since condition $9.12-b$ is implied by $10.5-b$, we may, by virtue of theorem 9.12 , conclude that:

- A period $\widetilde{T}>K$ exists such that all optimal solutions $((\hat{x}, \hat{y}),(\hat{u}, \hat{v}))$ of an infinite horizon problem satisfy:

$$
\left.\begin{array}{l}
(\tilde{v}, \tilde{u})^{\prime}(\hat{x}(t), \hat{y}(t))=0 \\
(\tilde{y}, \tilde{x})^{\prime}(\hat{u}(t), \hat{v}(t))=0
\end{array}\right\} t \geq \hat{T},
$$

- All optimal solutions $((\hat{\mathbf{x}}, \hat{y}),(\hat{\mathrm{u}}, \hat{\mathrm{v}}))$ of the infinite horizon problem converge in the following manner

$$
\left.\begin{array}{l}
\left(\frac{1}{\rho}\right)^{t}(\hat{x}(t), \hat{y}(t)) \rightarrow(\hat{x}, \tilde{y}), t \rightarrow \infty \\
\left(\frac{1}{\pi}\right)^{t}(\hat{u}(t), \hat{v}(t)) \rightarrow(\hat{u}, \tilde{v}), t \rightarrow \infty
\end{array}\right\} .
$$

Moreover, by virtue of theorem 6.21, we may conclude that numbers $M_{1}$ and $M_{2}$ exist, such that all optimal.solutions $((\hat{x}, \hat{y}),(\hat{u}, \hat{v}))$ of the infinite horizon problem satisfy:

$$
\begin{aligned}
& \hat{\mathrm{x}}_{1 / \rho} \in \sigma_{\infty}^{\mathrm{n}}\left(M_{1}\right) \\
& \hat{\mathbf{u}}_{1 / \pi} \in \sigma_{\infty}^{\mathrm{m}}\left(M_{2}\right)
\end{aligned}
$$

With respect to the positive numbers $\delta_{1}$, $\delta_{2}$ appearing in the associated Einite horizon problem, one can derive from (10.5.1) to (10.5.3), and from proposition $9.8-\mathrm{g}, \mathrm{h}$, that a period $\mathrm{T}^{*} \geqq$ exists such that all optimal $((\hat{x}, \hat{y}),(\hat{u}, \hat{v}))$ of the infinite horizon problem satisfy: 


$$
\left.\begin{array}{l}
\left\|\hat{x}(T)-\rho^{T} \tilde{x}\right\|_{\infty}<\rho^{T} \delta_{1} \\
\| \hat{u}(T)-\pi^{T} \tilde{u}_{\infty}<\pi^{T} \delta_{2}
\end{array}\right\} T \geq T^{*} .
$$

Now, consider a finite horizon problem $\overline{\mathrm{LP}}(\mathrm{T})$ with $\mathrm{T} \geqq \mathrm{T}^{*}$. For every optimal solution $((\hat{x}, \hat{y}),(\hat{u}, \hat{v}))$ of the infinite horizon problem we put:

$$
\begin{aligned}
& \hat{\mathrm{X}}:=\sum_{t=T^{T}+1}^{\infty} \pi^{t-T-1} S_{p}^{\prime} \hat{x}(t) . \\
& \hat{U}:=S_{d} \hat{u}(T+1),
\end{aligned}
$$

where $(10.5 .5)$ exists by virtue of the fact that $\hat{x}_{\pi} \in 1_{1}^{n}$. From $(10.5 .5)$ it follows:

$$
(\underline{B}-\pi \underline{A}) \hat{X}-S_{d} A \hat{x}(T)=\rho^{T+1} \sum_{t=0}^{\infty}(\rho \pi){ }^{t} S_{d} \hat{f}=\frac{\rho^{T+1}}{1-\rho \pi} S_{d} \tilde{f} . \quad(10.5 .7)
$$

Moreover, since $10.5-b$ implies that $\mathrm{D}^{r}=\mathrm{c}^{\mathrm{r}}, \mathrm{D}^{\mathrm{i}}=\mathrm{C}^{\mathrm{i}}$ (viz.10.2.1), it follows from $(10.5 .1),(10.5 .2),(10.5 .5),(10.5 .6)$, and from proposition $9.8-\mathrm{c}, \mathrm{d}$, that:

$$
\left.\begin{array}{l}
\left(x-\frac{\rho^{T+1}}{1-\rho \pi} S_{p}^{\prime} \tilde{x}\right) \in x^{0} \\
\left(\underline{B}^{\prime}-\pi A^{\prime}\right)\left(U-\pi^{T+1} S_{d} u\right) \in U^{0}
\end{array}\right\} .
$$

By virtue of proposition 10.3 , this implies the existence of vectors $\hat{z}^{r}, \hat{z}^{i}, \hat{w}^{r}$, and $\hat{w}^{i}$ satisfying:

$$
\left.\begin{array}{rl}
\hat{X}+C^{r} \hat{z}^{r}-C^{i} \hat{z}^{i} & =\frac{\rho^{T+1}}{1-\rho \pi} S_{p}^{\prime} \tilde{x} \\
c^{i} \hat{z}^{r}+C^{r} \hat{z}^{i} & =0
\end{array}\right\}
$$




$$
\left.\begin{array}{l}
\left(\underline{B}^{\prime}-\pi \underline{A}^{\prime}\right) \hat{U}+\hat{w}^{r}=\pi^{T+1}\left(\underline{B}^{\prime}-\pi \underline{A}^{\prime}\right) S_{d} \tilde{u}=\pi^{T+1} S_{p}^{\prime} \hat{p} \\
c^{r} \hat{w}^{r}+c^{i, \hat{w}^{i}}=0 \\
-c^{i, \hat{w}^{r}+C^{r} \cdot \hat{w}^{i}}=0
\end{array}\right\}
$$

From $(10.5 .4)$ to $(10.5 .9)$, we may conclude that vectors $\hat{Y}^{1}=0$, $\hat{\mathrm{Y}}^{2}=0, \hat{\mathrm{V}}^{1}=0, \hat{\mathrm{V}}^{2}=0, \hat{\mathrm{x}}^{1}, \hat{\mathrm{x}}^{2}, \hat{\mathrm{u}}^{1}, \hat{\mathrm{u}}^{2}>0$ exist such that $\left(\{(\hat{x}(t), \hat{y}(t))\}_{1}^{T}, \hat{x}, \hat{y}^{1}, \hat{y}^{2}, \hat{x}^{1}, \hat{x}^{2}, \hat{z}^{r}, \hat{z}^{i}\right)$ satisfies $(10.4 .2)$ and such that $\left.\left.((\hat{u}(t), \hat{v}(t))\}_{i}^{T}\right), \hat{U}, \hat{v}^{1}, \hat{v}^{2}, \hat{u}^{1}, \hat{u}^{2}, \hat{w}^{r}, \hat{w}^{i}\right)$-satisfies $(10.4 .4)$. Moreover, since $\hat{Y}^{1}=0, \hat{Y}^{2}=0, \hat{V}^{1}=0, \hat{V}^{2}=0$, it appears that $(10.4 .5)$ is satisfied. Thus, we may conclude that $\left(\{(\hat{x}(t), \hat{y}(t))\}_{1}^{T},\{(\hat{u}(t), \hat{v}(t))\}_{1}^{T}\right)$ is an optimal solution of $\overline{L P}(T)$. Hence, condition 10.4-a of the definition of equivalence is satisfied.

To prove that condition $10.4-b$ is satisfied, we assume that. $\{(x(t), y(t))\}_{1}^{\infty}$ is a primal optimal solution of $\overline{L P}(T)$, and denote $X, Y^{l}, Y^{2}, x^{l}, x^{2}, z^{r}, z^{i}$ as the vectors such that: $\left(\left\{(x(t), y(t)]_{1}^{T}, X, Y^{l}, Y^{2}, x^{1}, x^{2}, z^{r}, z^{i}\right)\right.$ satisfies $(10.4 .2)$.

Since $\left.(\hat{u}(t), \hat{v}(t)))_{1}^{T}, \hat{U}, \hat{v}^{1}, \hat{v}^{2}, \hat{u}^{1}, \hat{u}^{2}, \hat{v}^{r}, \hat{w}^{i}\right)$ is a dual optimal solution of $\overline{\mathrm{LP}}(\mathrm{T})$ for which $\hat{\mathrm{u}}^{1}, \hat{\mathrm{u}}^{2}>0,(10.4 .5)$ implies

$$
\left.\begin{array}{l}
(\hat{v}(t), \hat{u}(t))^{\prime}(x(t), y(t))=0, t=1, \ldots, T \\
Y^{\prime}=0, Y^{2}=0
\end{array}\right\} .
$$

So, from (10.4.2) and from proposition 10.3 , we may conclude:

$$
\begin{aligned}
& (\underline{B}-\pi \underline{A}) x-\underline{A S}_{p}^{\prime} x(T)=\frac{\rho^{T+1}}{1-\rho \pi} S_{d}, \\
& \left(X-\frac{\rho^{T+1}}{1-\rho \pi} S_{p}^{\prime} x\right) \in x^{\circ},
\end{aligned}
$$




$$
\left\|\left(\frac{1}{\rho}\right)^{T} \times(T)-x\right\|_{\infty} \leq \delta_{1}
$$

Since:

$$
s_{p}^{r y}=\left(\underline{B}-\frac{1}{p} A\right)^{-1} s_{d} \tilde{F},
$$

the equality $(10.8 .11)$ implies:

$$
(\underline{B}-\pi \underline{A})\left(X-\frac{\rho^{T+1}}{1-\rho \pi} S_{p}^{\prime} \tilde{x}\right)=A_{p}^{\prime}\left(x(I)-\rho^{T} \tilde{x}\right) .
$$

With the help of matrix $H$ defined by (9.6.4), the latter equality can be reduced to:

$$
X-\frac{p^{T+1}}{1-\rho \pi} S_{p}^{\prime} x=\frac{1}{\pi}(H-I) S_{p}^{\prime}\left(x(T)-\rho_{x}^{T} x\right)
$$

and with the help of the matrices $\Lambda$ and $Q$ defined by (9.6.7) to:

$$
Q^{-1}\left(X-\frac{\rho^{T+1}}{1-\rho \pi} S_{p}^{\prime} x\right)=\frac{1}{\pi}(\Lambda-I) Q^{-1} S_{p}^{\prime}\left(x(T)-\rho^{T} x_{2}\right) .
$$

From $(10.5 .14,(10.5 .12)$, and from definition $(9.6 .17)$ one can derive:

$$
s_{p}^{\prime}\left(x(T)-\rho^{T} x\right) \in x^{0}
$$

By virtue of proposition $9.8-e$, the relation $(10.5 .15)$ implies the existence of a sequence $\left(\{x(t)\}_{T+1}^{\infty},\{y(t)\}_{T+1}^{\infty}\right) \in 1^{n_{x 1}}$ m such that $\left(\{x(t)\}_{T}^{\infty},\{y(t)\}_{T}^{\infty}\right) \in \underline{X Y}$ (def. 9.6.21). Moreover, by virtue of $(10.5 .13)$ and proposition $9.8-i$, this implies

$$
x(t), y(t) \geqq 0, t \geqq T+1
$$

Thus we may conclude that $(x, y):=\left(\{x(t)\}_{1}^{\infty},\{y(t)\}_{1}^{\infty}\right)$ is a primal feasible solution of the infinite horizon problem. Moreover, from $(10.5 .1),(10.5 .10)$, and $(10.5 .6)$ we may conclude:

$$
\langle\hat{\mathrm{v}}, \mathrm{x}\rangle_{\infty}+\langle\hat{\mathrm{u}}, \mathrm{y}\rangle_{\infty}=0,
$$


and by virtue of $\left(\{x(t)\}_{T+1}^{\infty},\{y(t)\}_{T+1}^{\infty}\right) \in \underline{X Y}$ (def. 9.6 .21$)$ :

$$
x_{1 / \rho} \in 1_{\infty}^{\mathfrak{n}}, y_{1 / \rho} \in 1_{\infty}^{\mathrm{m}}
$$

Thus by theorem 6.21 , it appears that ( $x, y)$ is a primal optimal solution of the infinite horizon problem. This proves that, with respect to the primal problem, condition 10.4-b is satisfied.

Now, we assume that $\{(u(t), v(t)))_{1}^{T}$ is a dual optimal solution of $\overline{L P}(T)$. In a similar manner as the relations $(10.5 .11)$ to $(10.5 .13)$ are found, we may conclude that a $U$ exists such that:

$$
\begin{aligned}
& B^{\prime} u(T)-A^{\prime} S_{d}^{\prime} U=\pi^{T}{ }_{p}^{\prime}, \\
& \left(B^{\prime}-\pi A^{\prime}\right)\left(U-\pi^{T+1} S_{d} \tilde{u}\right) \in U^{0}, \\
& \left\|\left(\frac{1}{\pi}\right)^{T+1} U-S_{d} \tilde{u}\right\|_{\infty} \leqq \delta_{2} .
\end{aligned}
$$

Now, by virtue of proposition $9.8-f$, the relation $(10.5 .18)$ implies the existence of a $\left(\{u(t)\}_{T+1}^{\infty},\{v(t)\}_{T+1}^{\infty}\right) \in \underline{U V}$ (def. 9.6.22) such that $u(T+1)=S_{d}^{\prime} U$. Moreover, by virtue of $(10.5 .19)$ and proposition $9.8-j$, we may conclude

$$
u(t), v(t) \geqq 0, t \geqq T+1
$$

With the help of this result, the proof with respect to the dual optimal solution may be completed in a similar manner as done for the primal optimal solution.

\subsection{Remark.}

If no eigenvalues $\lambda_{i i}$ exist for which

$$
\left|\frac{\lambda_{i i}-1}{\lambda_{i i}}\right| \leqq 1,
$$


then the matrices $c^{r}, C^{i}, D^{r}$, and $D^{i}$ do not exist. In this case the sets $X^{\circ}$ and $U^{\circ}$ (def. $10.2-c, d$ ) are defined by $X^{\circ}:=\{0\}$ and $\mathrm{u}^{\mathrm{o}}:=\mathrm{R}^{\mathrm{k}}$.

The associated problems over a finite horizon can be written:

$$
\begin{aligned}
\phi\left(x, X, Y^{1}, Y^{2} ; T\right): & =\left\langle p_{\pi}, x\right\rangle_{1, T}+\pi^{T+1} \tilde{p}^{\prime} s_{p} X+ \\
& +\pi^{T+1}\left(\tilde{u}-\delta_{2} e\right)^{\prime} S_{d} Y^{1}+\pi^{T+1}\left(\tilde{u}+\delta_{2} e\right) S_{d}^{\prime} Y^{2},
\end{aligned}
$$

to be maximalized over the vectors $\{(x(t), y(t))\}_{1}^{T}, X, Y^{1}, Y^{2}$, $x^{1}, x^{2}$ which satisfy

$$
\begin{aligned}
& B \times(1)+y(1)=\rho f^{\circ}(1) \\
& B x(t+1)-A x(t)+y(t+1)=\rho^{t+1} f^{\circ}(t+1), t=1, \ldots, T-1 \\
& (\underline{B}-\pi \underline{A}) X-S_{d} A x(T)+Y^{1}-Y^{2}=\frac{\rho^{T+1}}{1-\rho \pi} S d^{T} \\
& x(T)+x^{1}=\rho^{T}\left(x+\delta_{1} e\right) \\
& -x(T)+x^{2}=\rho^{T}\left(-\tilde{x}+\delta \delta_{l} e\right) \\
& x=\frac{p^{T+1}}{1-p \pi} S_{p}^{\prime} x \\
& x(t), y(t) \geqq 0, t=1, \ldots, r \\
& \mathrm{x}^{1}, \mathrm{x}^{2}, \mathrm{y}^{1}, \mathrm{y}^{2} \geq 0
\end{aligned}
$$

and the corresponding dual problem:

$$
\begin{aligned}
\psi\left(u, v, v^{1}, v^{2}, w^{r} ; T\right): & =\left\langle f_{\rho}^{0}, u\right\rangle{ }_{1, T}+\frac{\rho^{T+1}}{1-\rho \pi^{\prime}} s_{d}^{\prime} U+\rho^{T}\left(x^{u}+\delta_{1} e\right)^{\prime} v^{l}+ \\
& +\rho^{T}\left(-\tilde{x}^{2}+\delta_{1} e\right)^{\prime} v^{2}+\frac{\rho^{T+1}}{1-\rho \pi} x^{\prime} s_{p} w^{r}
\end{aligned}
$$


to be minimalized over the vectors $\{(\mathrm{u}(\mathrm{t}), \mathrm{v}(\mathrm{t}))\}_{\mathrm{J}}^{\mathrm{T}}, \mathrm{U}, \mathrm{v}^{1}, \mathrm{v}^{2}$, $u^{1}, u^{2}, w^{r}$, which satisfy:

$$
\begin{aligned}
& B^{\prime} u(t)-A^{\prime} u(t+1)-v(t) \quad=\pi^{t} p(t), t=1, \ldots, T-1 \\
& \cdot B^{\prime} u(T)-A^{\prime} S_{d}^{1} U+V^{\prime}-V^{2}-v(T)=\pi^{T}{ }_{p}^{n} \\
& u-u^{\prime}=\pi^{T+1} S_{d}\left(\hat{u}-\delta_{2} e\right) \\
& -U-u^{2}=\pi^{T+1} S_{d}\left(-\tilde{u}-\delta_{2} e\right) \\
& \left(\underline{B}^{\prime}-\pi A^{\prime}\right) U+w^{r}=\pi^{T+1} S_{p}^{\prime} \tilde{p} \\
& u(t), v(t) \geqq 0, t=1, \ldots, T \\
& u^{1}, u^{2}, v^{1}, v^{2} \geq 0
\end{aligned}
$$

It can be shown that in this case too, theorem 10.5 is valid.

A similar situation may also arise if the non-degenerated equilibrium combination $(\tilde{x}, \tilde{y}),(\tilde{u}, \tilde{v})$ is such that $\tilde{x}=0$ and $\tilde{u}=0$. clearly, in that case the only semi equilibrium path possible is :

$$
\left.\begin{array}{l}
(x(t), y(t))=\rho^{t}(0, \tilde{f}) \\
(u(t), v(t))=\pi^{t}\left(0, \frac{u}{p}\right)
\end{array}\right\} t \geq 1 .
$$

\subsection{Remark.}

If the conditions of theorem 10.5 have been satisfied, then it appears that all optimal solutions of the infinite horizon problem can be found with the help of a linear programming problem in a finite dimensional space. Basically, such an infinite horizon problem is hereby solved.

In the solving procedure three phases may be discerned: finding the equilibrium combination, setting up the associated linear 
programming problem over a finite horizon according to $\$ 10.4$ or $\$ 10.6$ and, finally, solving the finite horizon problem.

Concerning the finding of equilibrium combinations, proposition 8.3 suggests a pametric approach of the linear programing problem defined in $\$ 8.2$.

When an equilibrium combination has been found satisfying the requirements, then matrices $c^{r}$ and $c^{i}$ (def: 10.2-a) can be found by calculating the eigenvalues and eigenvectors of matrix H (def. 9.6 .4 ).

The best way to solve the associated programming problem over a finite horizon seems to be using a parametric process, in. its essence deduceble from the proof of theorem 9.12 (viz. def. 9.12 .6 , def. 9.12 .16 , and property 13 of this theorem). 


\section{REFERENCES.}

1. Berge, C: Espaces topologiques, fonctions multivoques, Paris: Dunod, 1959.

2. Dorfman, R; Samuelson, P.A.; Solow, R.M.: Linear programming and economic analysis, New York: MeGraw-Hill Book Comp, 1958.

3. Gantmacher, F.R.: The theory of matrices, New York: Chelsea Publishing Company: 1959.

4. Koopmans, T.C.: Three essays on the state of economic science, New York: McGraw-Hi11 Book Company, 1957.

5. Luenberger, D.G.: Optimization by vector space methods, New York: John Wiley and Sons: 1969.

6. Nicado, H.: Convex structures and economic theory, New York: Academic Press, 1968.

7. Ortega, J.M.; Rheinboldt. W.C.: Iterative solution of nonlinear equations in several variables, New York: Academic Press: 1970.

8. Rockafellar, R.T.: Convex analysis, Princeton: Princeton University Press, 1970.

9. Schumann, J.: Input-output-analyse, Berlin-Heidelberg; Springer-Ver1ag, 1968.

10 Van Slyke, R.M.; Wets, R.J.B.: A duality theory for abstract mathematical programs with applications to optimal control theory, J. Math, Ana1. App1. Vo1 22 (1968), No. 3, 679-706.

11 Von Neumann, J.: A model of general economic equilibrium, Review of economic studies, $13(1945-1946), 1-9$. 


\section{LIST OF SYMBOLS.}

Spaces.

$R^{n}$ : real n-dimensional vector space,

$R_{+}^{n}:=\left\{x \in R^{n} \mid x_{i} \geq 0, i=1,2, \ldots, n\right\}$.

$1^{k}, 1_{1}, 1_{\infty}, 1_{*}^{k}: 24,25$.

$c_{0}, c_{0}^{k}: 89$.

$\|x\|,\|x\|^{T, K}: 24,25,26$

$x_{i} \rightarrow x_{0}, i \rightarrow \infty$ : convergence of sequence $\left\{x_{i}\right\}_{1}^{\infty}$ to $x_{0}$

$x_{i} \stackrel{x}{*}_{0}, i \rightarrow \infty$ : weak ${ }^{*}$ convergence, 89 .

\section{Sets and vectors.}

Int (C): interior of $C$.

$\bar{c}:$ closure of $c$.

$\pi(C)$ : power set of $C$.

$\sigma_{\infty}^{n}(M):$ sphere in $l_{\infty}^{n}$, with radius $M, \| 12$.

$X \times Y$ : cartesian product of sets $X$ and $Y$.

$X \odot Y:=\{z:=X+y \mid x \in X, y \in Z\}$, the sum of sets $X$ and $Y$.

$x>0: x$ is positive, i.e.: $x_{i}>0, i=1,2, \ldots$

$x \geq 0: x$ is non-negative, i.e.: $x_{i} \geq 0, i=1,2, \ldots$

$x \geq 0: \quad x \geq 0, \quad x \neq 0$

$[a, b]:=\{x \mid a \leq x \leq b\}$ closed interval

$[a, b[:=\{x \mid a \leq x<b\}$

]$a, b]:=\{x \mid a<x \leq b\}$

$(x, y)$ : the vector with components $\left(x_{1}, \ldots, x_{n}, y_{1}, \ldots, y_{m}\right)$.

$\{x(t)\}_{1}^{\infty}$ sequence of vectors $x(1), x(2), \ldots$

$\left\{x^{i}\right\}_{1}^{\infty}$ sequence of vectors $x^{1}, x^{2}, \ldots$ 
Mappings, functionals, and matrices.

$G: X \rightarrow Y:$ (single-valued) mapping from $X$ into $Y$

$G: X \rightarrow \pi(Y):$ set-valued mapping from $X$ into $Y$

graph $(C ; D): 1 / 2$

$x^{\prime} y$ : inner product of finite dimensional vectors $x$ and $y$

$\langle x, y\rangle=\sum_{t=1}^{\infty} x(t)^{\prime} y(t)$

$\langle x, y\rangle_{T, K}: \sum_{t=T}^{K} x(t)^{\prime} y(t)$

I : identity matrix

0 : zero aatrix

$\|A\|_{\infty}: 1_{\infty}^{-n o r m}$ of matrix A

$A^{\prime}$ : transpose of matrix A

$A^{-1}:$ inverse of matrix A. 


\section{SUBJECT INDEX.}

Consistence, 59 .

Directed, primal, dual, 36 .

Dominated $(\alpha-), 27,52,57$.

Dual spaces, 88 .

Dual system, 29 .

Equilibrium combination, 125,

$$
\text { non-degenerated, } 133 .
$$

Equilibrium path, 125 ,

$\operatorname{semi}, 133$.

Equivalent 1 inear programming problem, 169,175 .

Exponential, semi, 36 .

Farkas" theorem, 65 .

Feasible, virtual1y, 30 .

Graph, 112 .

Inferiority, 31,66

Initial vector, 28

Linear, functionals, 27,88 , programming problem, 33, programming system, 28 .

Objective function, 31 .

optimal solution, 32,85 *

Parametric properties, 111.

Primal system, 29 .
Regular, virtual1y, 30 .

slack variables, 28 .

Sphere, 112.

Stiemke's theorem, 54.

superregular, virtually, 31 .

Symmetry, 34 .

Transform $(\alpha-), 26$.

Transformed system, 35

Upper semicontinuous, 123.

Weak closedness, compactness, convergence, 89 . 


\section{STELLINGEN}

I

Bij de bewijsvoering betreffende de existentie van evenwichten in homogene lineaire modellen van economische systemen, wordt in de literatur algemeen de veronderstelling gemakt dat de daarin optredende matrices niet-negatief zijn.

Deze eis kan echter aanienlijk worden afgezwakt.

II

De existentie van Nash-evenwichten voor twee-personen-som-ongelijknul-spelen, volgt eenvoudig uit propositie 8.6 van dit proefschrift. (Litt. Cottle and Danzig: Complementary pivot theory of math. prog, Linear Algebra and its Aplications 1, 103-125, 1968).

\section{I I}

Onder bepalde voorwatden kan een evenwichts-kombinatie, zoals gedefinieerd in hoofdstuk 8 van dit proefschrift, berekend wor-, den met behulp van een algorithme gebaseerd op het volgende proces:

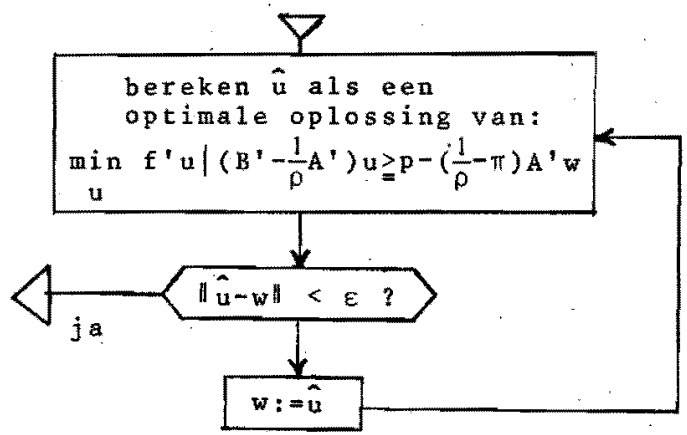


$z i j n C_{1}: z_{1} \rightarrow z_{2}$ en $C_{2}: z_{1} \rightarrow z_{2}$ twee lineaire transformaties op een eindig dimensionale vektorruimte $z_{1}$, wet de eigenschap dat voor iedere deelruimte $\mathrm{zCz}$, geldt:

$$
\text { dim }\left\{c_{1}(z)+c_{2}(z)\right\} \geqq d i m\{z\} \text {, }
$$

dan bestaat een vektorruimte $\tilde{Z} \subset z_{1}$ en een lineaire transfor-

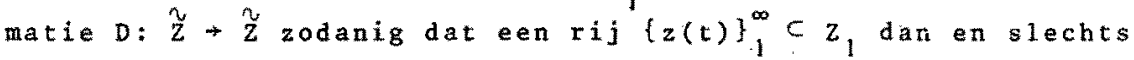
dan voldoet an

$$
c_{1}(z(t+1))=c_{2}(z(t)), t \geqq 1,
$$

als voldaan wordt an:

$$
\left.\begin{array}{c}
z(1) \in Z \\
z(t+1)=D(z(t)), t \geq 1
\end{array}\right\}
$$

v

De resultaten van dit proefschrift geven de indruk dat het bestan van economische evenwichtspaden sterk afhankelijk is van de hoogte van de diskonterings factor. In het bijzonder lijkt het er op dat een te lage diskonteringsfaktor de oorzak zou kunnen $z i j n$ yan schoksgewijze economische ontwikkelingen.

VI

Een a atal veronderstelingen welke relevant zijn voor transportverschijnselen van stoffen in leverweefsels geven anleiding tot het volgende stelsel differential vergelijkingen:

$$
\left.\begin{array}{l}
\frac{\partial A}{\partial t}=c_{a}\left\{(1-B) \frac{\partial^{2} A}{\partial x^{2}}+A \frac{\partial^{2} B}{\partial x^{2}}\right\} \\
\frac{\partial B}{\partial t}=c_{b}\left\{(1-A) \frac{\partial^{2} B}{\partial x^{2}}+B \frac{\partial^{2} A}{\partial x^{2}}\right\}
\end{array}\right\},
$$

warin $A(t, x)$ en $B(t, x)$ de konsentraties voorstellen van twee stoffen op tijdstipt en op plats $x$.

Een dergelijk stelsel kan van betekenis zijn voor het onderzoek van de leverfunkties met behulp van radioaktieve stoffen. 
Een samenwerkingsverband tussen de onderafdeling der Wiskunde van de Technische Hogeschool Eindhoven en de Economische Faculteit van de Katholieke Hogeschool Tilburg, zou gunstig zijn voor onderwijs en onderzoek zowel op het gebied van de toegepaste wiskunde als op het gebied van de economie.

\section{VIII}

Openbaar vervoer gebaseerd op een centraal bestuurd horizontaal iftensysteem moet in stat worden geacht het serviceniveau van taxi's te benaderen, tegen aanzienlijk lagere kosten. Het is daarom wenselijk dat ook in Nederland een onderzoekprograma voor de bestudering van dergelijke systemen wordt opgesteld.

J.J.M. Evers. 